\title{
Photochemical Effects on the Ices and Dust Grains of Astronomical Importance
}

\author{
Chunqing Yuan \\ Hefei, China
}

B.S. in Chemistry, University of Science and Technology of China, 2009

\author{
A Dissertation presented to the Graduate Faculty \\ of the University of Virginia in Candidacy for the Degree of \\ Doctor of Philosophy
}

Department of Chemistry

University of Virginia

May 2014 


\section{Abstract}

Dust grains and the ice layers condensed on the grains in the interstellar medium are important places for new molecules to form. This thesis aims to use laboratory experimental methods to investigate Lyman- $\alpha$ radiation $(10.2 \mathrm{eV})$ induced photochemical effects that occur on astronomical ices and dust grains. We perform experiments in an ultrahigh vacuum cell and use cryogenic cooling to mimic the interstellar environment. The reaction processes and products are studied by Fourier Transform Infrared Spectroscopy (FTIR), Quadrupole Mass Spectrometry (QMS), and other surface analysis methods.

We conducted a comprehensive study on the photochemical effects during the life cycle of interstellar $\mathrm{CO}_{2}$ ice, from its formation pathways to its photodissociation and photodesorption mechanisms. A new Eley-Rideal type mechanism of $\mathrm{CO}+\mathrm{OH}$ reaction is proposed to explain the $\mathrm{CO}_{2}$ ice formation in the ISM. This reaction process describes an incoming gas phase $\mathrm{CO}$ molecule directly react with a surface $\mathrm{OH}$ radical produced by photodissociation of $\mathrm{H}_{2} \mathrm{O}$ ice. We also studied the photodissociation of $\mathrm{CO}_{2}$ ice, and found an isotope effect which favors the light isotopomer, ${ }^{12} \mathrm{CO}_{2}$, to dissociate faster than ${ }^{13} \mathrm{CO}_{2}$, due to the difference in the probability of dissociating the $\mathrm{C}-\mathrm{O}$ bond in electronicallyexcited $\mathrm{CO}_{2}{ }^{*}$ molecules when in close contact with the ice matrix. In the studies of photodesorption of $\mathrm{CO}_{2}$ ice, we demonstrated the efficiency of $\mathrm{CO}_{2}$ desorption is strongly correlated inversely to the efficiency of $\mathrm{CO}_{2}$ vibrational relaxation in the ice. In addition, we show how the interaction between high energy photons and a $\mathrm{CO}_{2}$ ice lattice affects the photodesorption efficiency. Besides ices, we studied the UV-induced surface reactions of $\mathrm{N}_{2} \mathrm{O}$ molecules adsorbed on $\mathrm{SiO}_{2}$ grain surfaces. The grain surface is shown to favor association reactions in producing $\mathrm{NO}_{2}$ and $\mathrm{N}_{2} \mathrm{O}_{4}$, comparing to those reactions in the gas phase. The roles of surface functional groups and their effects on the reaction rates, such as cage effect, quenching and spacing effects are also studied. 


\section{Acknowledgement}

Five years ago, I came to Charlottesville with curiosity and hope, to start my graduate study. I truly love this beautiful small town, which has plenty of sunshine, historic buildings, and friendly people. I feel that I am very lucky to have the memorable and fruitful five years here. I would like to express my deepest appreciation to my advisor, Professor John T. Yates, Jr., who is an excellent scientist and teacher. As amateur astronomers, we started our scientific exploration on the astrochemistry. His brilliant ideas, critical thinking, and hardworking attitude greatly influenced my way of doing research. Without his supervision and help, this thesis would not have been possible.

I would also thank Professor Eric Herbst, who is a distinguished astrochemist, for giving us many insightful suggestions in this area. My advisory committee members, Prof. Brooks Pate, Prof. Kevin Lehmann, Prof. Ian Harrison and Prof. Kelsey Johnson, also provide valuable comments on this thesis. I especially thank Dr. Mahesh Rajappan, who taught me everything about the apparatus and experimental methods. Dr. Zhen Zhang gave me a lot of help whenever I had trouble, and he always had great ideas to solve the problem. My lab members, Izzie, Lynn, Ana, Monica, Ilsa and Shiliang, are all wonderful colleagues. We learned a lot from each other, and we shared our happiness together. I also thank the Department of Chemistry, for providing me financial support and fellowship, and for the helpful and kind staffs.

Finally, I want to thank my parents, who always stand behind me and give me support whenever I need them. I thank my girlfriend, for all your love, support and patience. I also thank my friends, for all the happy moments we were together. We cook Chinese food, play games, go hiking and watch the stars. They made my life full of joy and I never felt alone. 


\section{Table of Contents}

1 Chapter 1: Introduction ................................................................................... 1

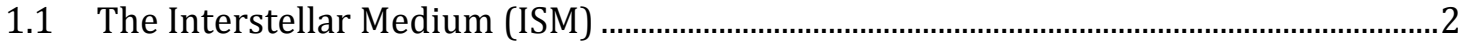

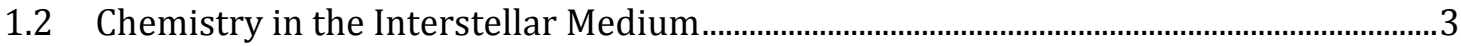

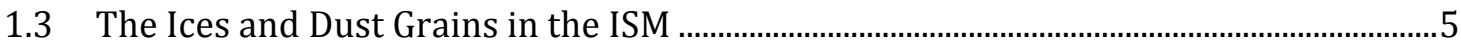

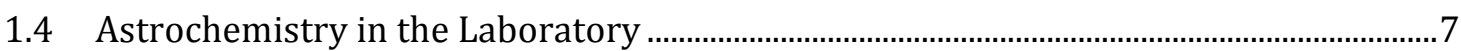

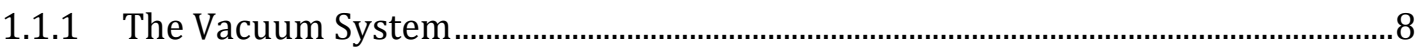

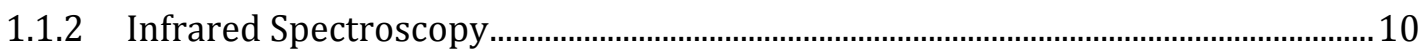

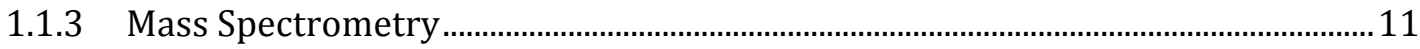

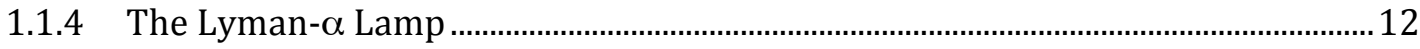

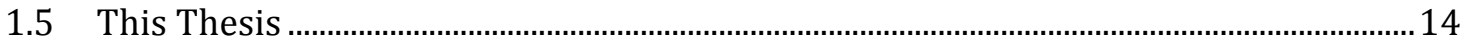

1.1.5 Photochemical Effects on the Life Cycle of Interstellar $\mathrm{CO}_{2}$ Ices ............................15

1.1.6 Surface Reactions on Silicate Dust Grains ...................................................................19

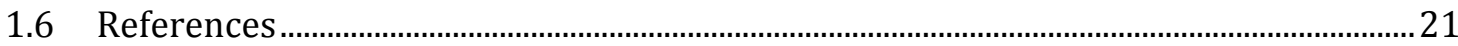

2 Chapter 2: A New Source of $\mathrm{CO} 2$ in the Universe: A Photoactivated Eley-

Rideal Surface Reaction on Ices ............................................................................ 26

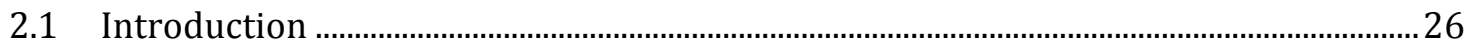

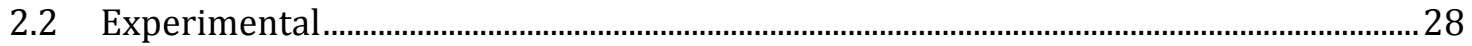

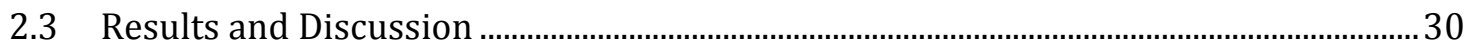

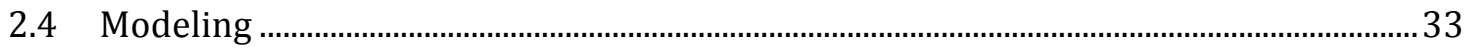

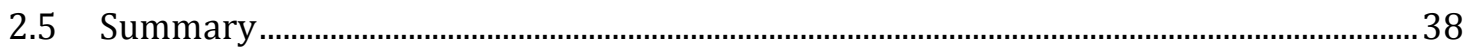

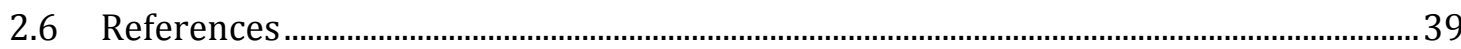

3 Chapter 3: Isotope Effect in the Photochemical Decomposition of $\mathrm{CO}_{2}$ (ice) by

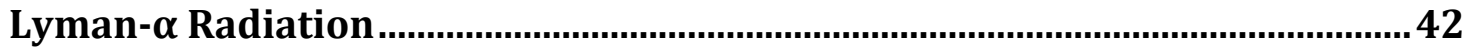

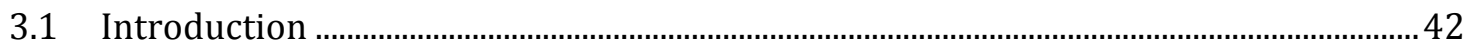

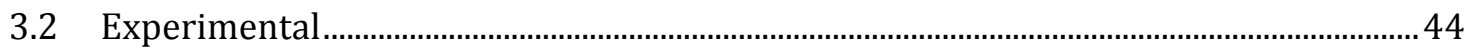

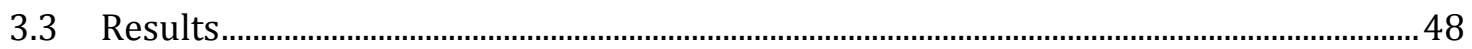

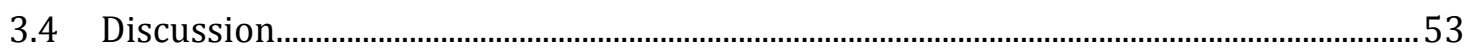

3.4.1 Origin of the Isotope Effect in Photoexcitation — Qualitative ...................................53 
3.4.2 Detailed Considerations of the Origin of the Isotope Effect-Connection to the Menzel-Gomer-Redhead Model of Electron Stimulated Desorption from

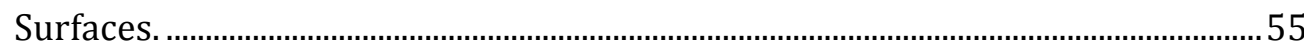

3.4.3 Overall Kinetic Model for Photodissociation and Photodesorption of $\mathrm{CO}_{2}$ ice...57

3.4.4 Efficiency of Photodesorption from $\mathrm{CO}_{2}$ ice at $75 \mathrm{~K}$ by Lyman- $\alpha$ Radiation ........59

3.4.5 Summary of Relative Cross Sections for All Processes...............................................60

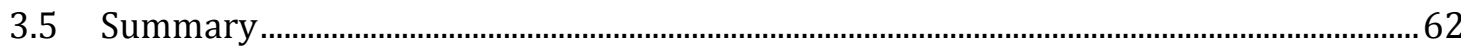

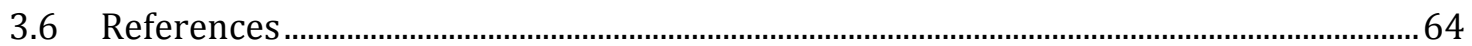

4 Chapter 4: Lyman- $\alpha$ Photodesorption from $\mathrm{CO}_{2}$ (ice) at $75 \mathrm{~K}$ : Role of $\mathrm{CO}_{2}$ Vibrational Relaxation on Desorption Rate...................................................67

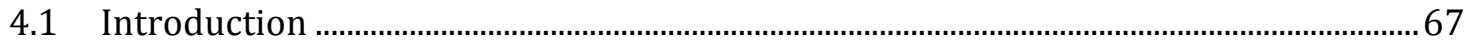

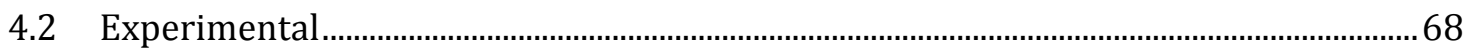

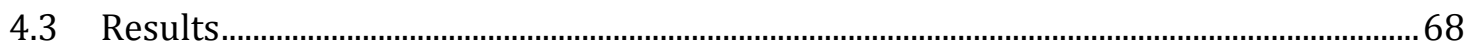

4.3.1 Photodesorption from 50\%-50\% ${ }^{12} \mathrm{CO}_{2}{ }^{-13} \mathrm{CO}_{2}$ Mixed Ice............................................. 68

4.3.2 Photodesorption from 75\%-25\% ${ }^{12} \mathrm{CO}_{2}-13 \mathrm{CO}_{2}$ Mixed Ice.......................................... 71

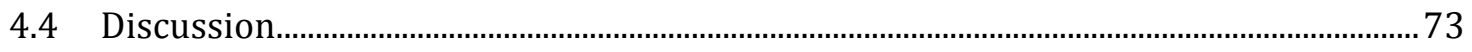

4.4.1 Photochemical Processes in $\mathrm{CO}_{2}$ (ice)-General .........................................................

4.4.2 Vibrational Tuning of the Ice Matrix to Control $\mathrm{CO}_{2}\left(\mathrm{~V}^{*}\right)$ Relaxation and

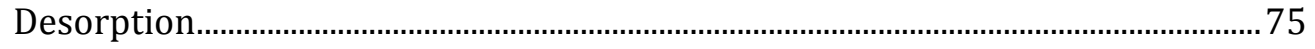

4.4.3 Details of Vibrational Relaxation and Photodesorption in $\mathrm{CO}_{2}$ Ice...........................77

4.4.4 Efficiency of Vibrational Relaxation in $\mathrm{CO}_{2}$ (ice) .......................................................... 79

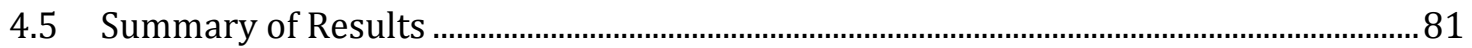

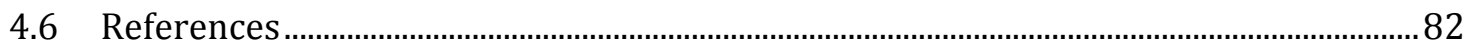

5 Chapter 5: Radiation Damage and Associated Phase Change Effect on

Photodesorption Rates from Ices - Lyman- $\alpha$ Studies of the Surface

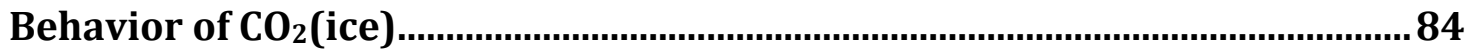

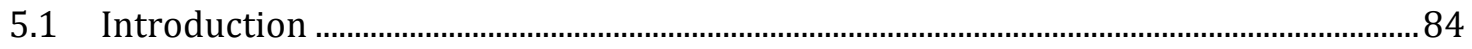

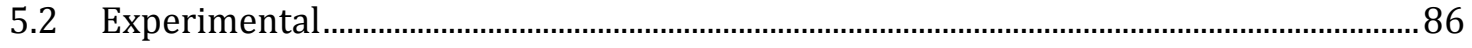

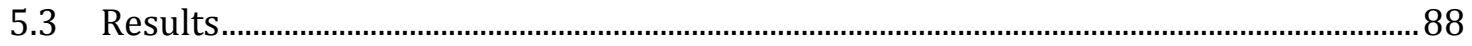

5.3.1 Kinetics of Processes in $\mathrm{CO}_{2}$ (ice) Induced by Lyman- $\alpha$ Irradiation..........................88

5.3.2 Spectroscopic Observations of ${ }^{13} \mathrm{CO}_{2}$ (ice) Damage by Lyman- $\alpha$ Radiation ......... 96 
5.3.3 Detection of the Depth of Radiation Damage in ${ }^{13} \mathrm{CO}_{2}$ (ice) by Lyman- $\alpha$

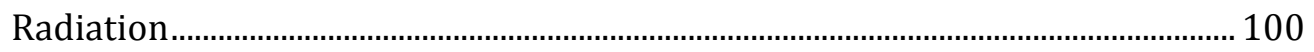

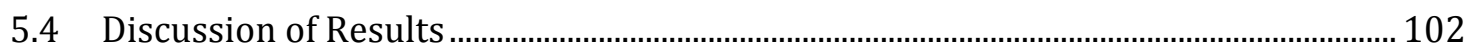

5.4.1 $\mathrm{CO}$ and $\mathrm{CO}_{2}$ Photo- and Thermal-Desorption from $\mathrm{CO}_{2}$ (ice) at $75 \mathrm{~K}$.................. 102

5.4.2 Gas Phase Kinetic Measurements versus Bulk IR Measurements in $\mathrm{CO}_{2}$ (ice)Separating Surface Processes from Bulk Processes.............................................. 104

5.4.3 Deep Defect Formation in $\mathrm{CO}_{2}$ (ice) by Lyman- $\alpha$ Radiation- The Provision of New Transport Pathways to the Ice Surface ........................................................... 105

5.4.4 Synopsis of Photo Processes in Radiation-Damaged $\mathrm{CO}_{2}$ (ice) ............................. 109

5.4.5 Astronomical Implications .......................................................................................... 112

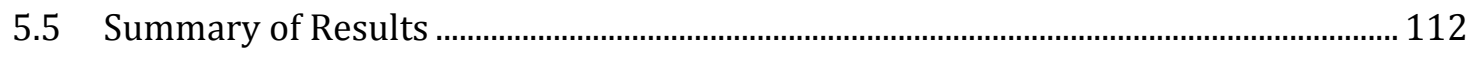

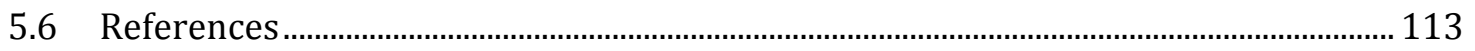

\section{Chapter 6: Lyman- $\alpha$ Driven Molecule Formation on $\mathrm{SiO}_{2}$ Surfaces-} Connection to Astrochemistry on Dust Grains in the Interstellar Medium.

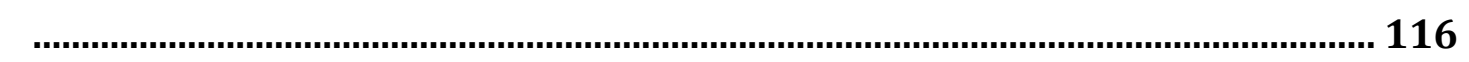

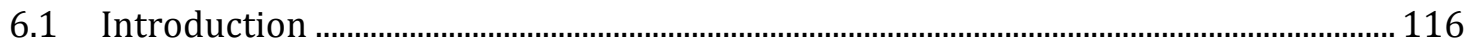

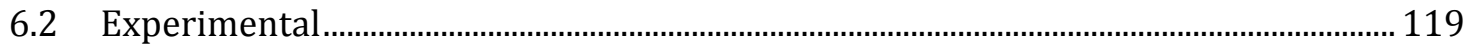

6.2.1 Lyman- $\alpha$ flux at $\mathrm{SiO}_{2}$ sample ..................................................................................... 119

6.2.2 $\mathrm{SiO}_{2}$ Sample Preparation and Mounting.................................................................. 121

6.2.3 Control Experiments - Heating by Lyman- $\alpha$ Lamp.................................................. 122

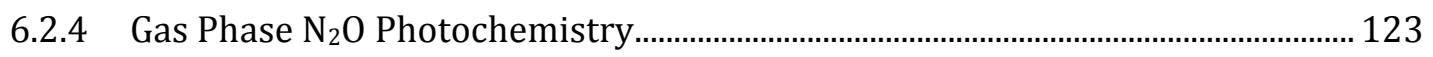

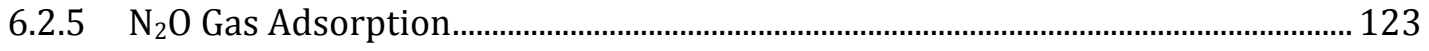

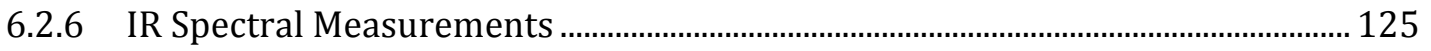

6.2.7 The Availability of Surface Sites for Photochemistry - Comparison of Gas Phase to Adsorbed Phase Measurements ............................................................................... 126

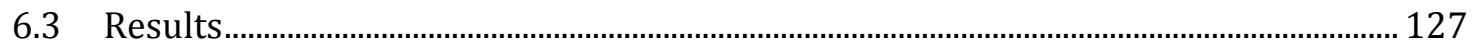

6.3.1 Photo-stability of Si-OH Groups by Lyman- $\alpha$ Radiation ........................................ 127

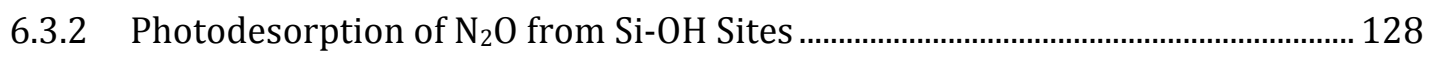

6.3.3 Qualitative Comparison of Gas Phase and Surface Phase Photo- decomposition

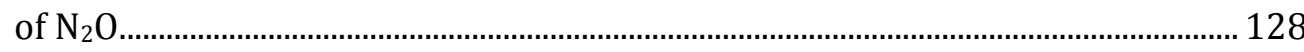

6.3.4 Quantitative Measurement of the Photochemical Rates by Infrared

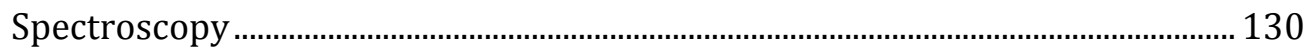


6.3.5 Quantum Efficiency of $\mathrm{N}_{2} \mathrm{O}$ Photodecomposition on High Area $\mathrm{SiO}_{2}$ 135

6.4 Discussion. 136

6.4.1 Efficiency of Complex Molecule Formation from Lyman- $\alpha$ Photo-decomposition of $\mathrm{N}_{2} \mathrm{O}$... 136

6.4.2 Decreasing Combination Reaction Rates as Photodecomposition Increases .. 139

6.5 Summary of Results 140

6.6 References 141

\section{Chapter 7: Photochemical Decomposition of Adsorbed $\mathrm{N}_{2} \mathrm{O}$ on Simulated} Silicate Interstellar Dust Grains using Lyman- $\alpha$ (10.2eV) Irradiation-The Role of Surface Functionalization of the $\mathrm{SiO}_{2}$ Substrate ................................ 145

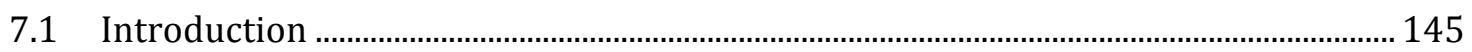

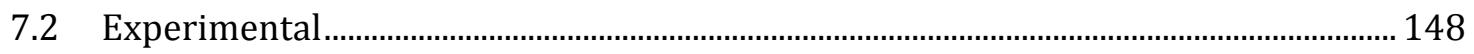

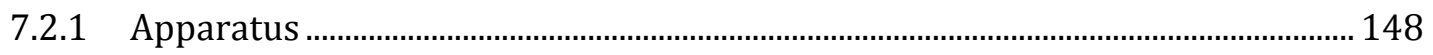

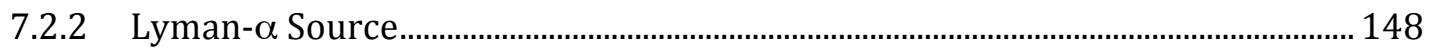

7.2.3 $\mathrm{SiO}_{2}$ Surface Modifications and $\mathrm{N}_{2} \mathrm{O}$ Adsorption ....................................................... 149

7.2.4 Stability of Si-OH and Si-O-SiCl 3 toward Lyman- $\alpha$ irradiation .............................. 151

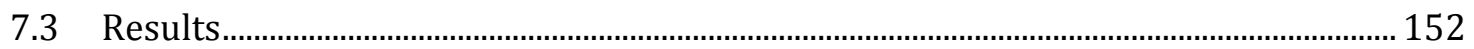

7.3.1 Typical Spectral Observation of Photochemical Product Formation .................. 152

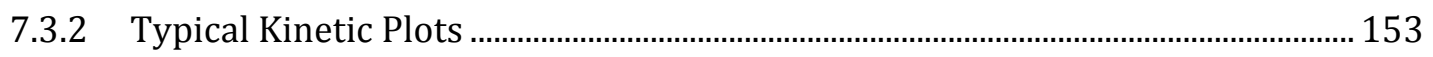

7.3.3 Reduced Initial Rate of Association Product Formation ......................................... 154

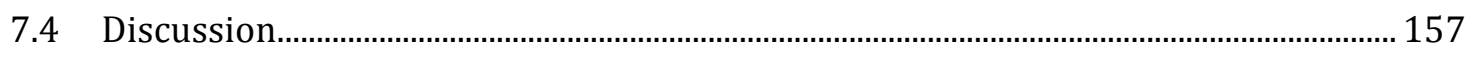

7.4.1 Steric Screening of Association Reaction by Surface Functionalities ................... 157

7.4.2 Quenching of the Electronically Excited States ........................................................... 157

7.4.3 Indirect Photochemistry Involving Electronic Excitation of $\mathrm{SiO}_{2} \ldots \ldots \ldots \ldots \ldots \ldots \ldots \ldots . . . . . . . . .158$

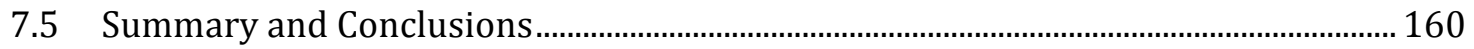

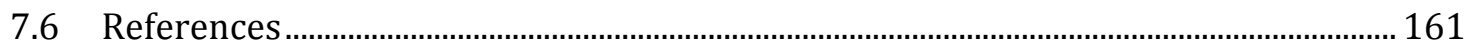

\section{Appendix I: Spectroscopic Observations of the Dynamics of the}

Displacement of Physically Adsorbed Molecules- CO on $\mathrm{C}_{60} \ldots \ldots \ldots \ldots \ldots \ldots . . . . . . . . . . .163$

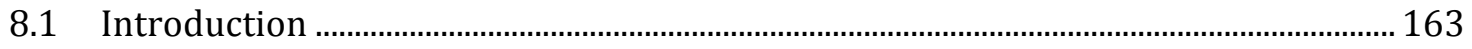

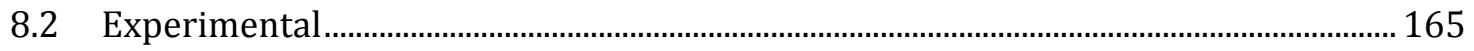

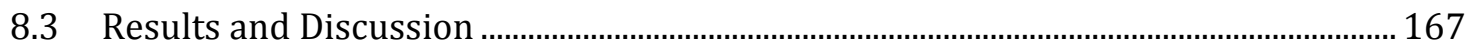




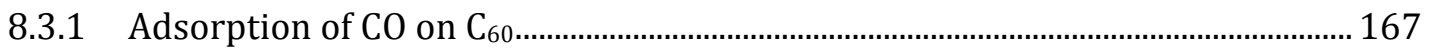

8.3.2 Displacement of Physisorbed CO by He, Ne and Ar................................................ 169

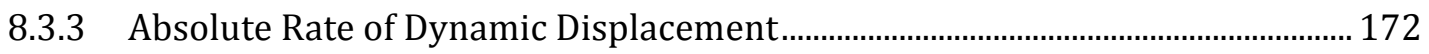

8.3.4 Displacement of Physisorbed CO by Kr ..................................................................... 172

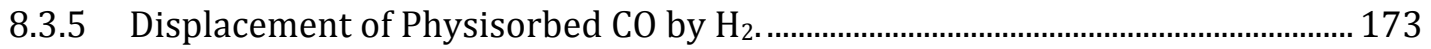

8.3.6 Temperature Dependence of Physisorbed CO Displacement by Ar..................... 174

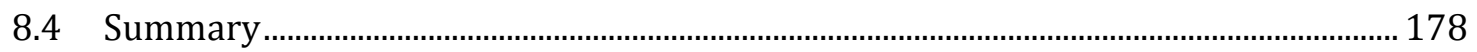

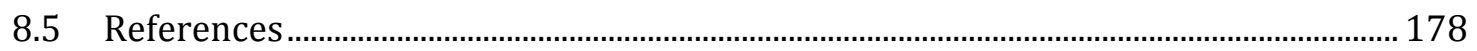

9 Appendix II: Experimental Details ………................................................. 180

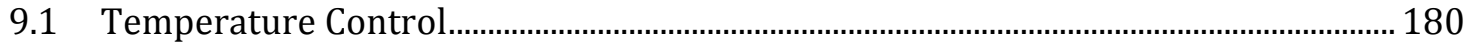

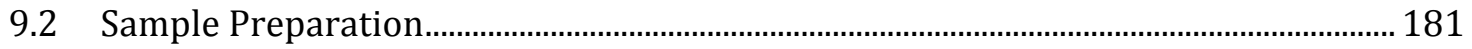

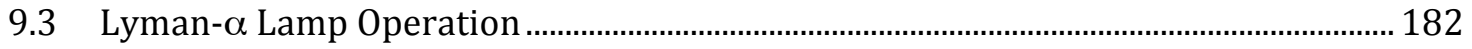

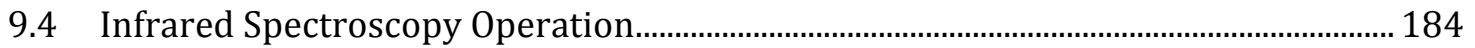

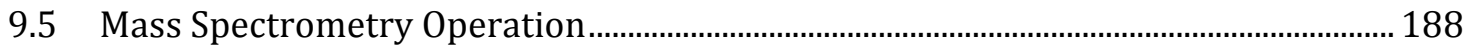

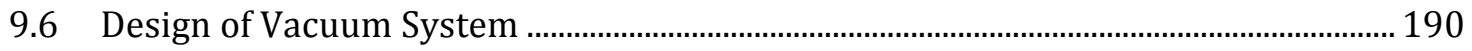

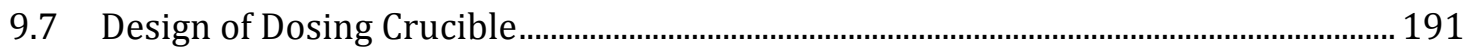

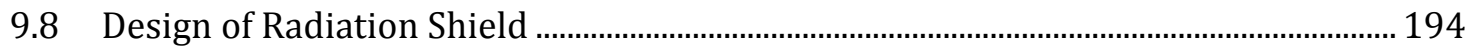

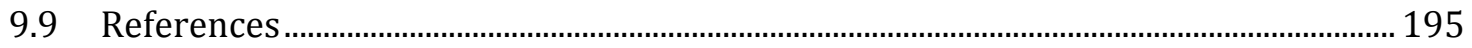




\section{Chapter 1: Introduction}

The Universe is full of mysteries. Human beings have been interested in exploring space since ancient times. One of the central questions is the origin of life. How have complex creatures evolved, in billions of years, from simple molecules? The discovery of over 180 molecules in the interstellar medium (ISM) suggests that the first molecules be synthesized in the interstellar environment.

The interstellar medium comprises about $10-15 \%$ of the total mass of the Galaxy [1, 2]. Approximately $99 \%$ of the mass of the ISM is in the form of gas, and the other $1 \%$ is in the form of dust grains (small particles usually made of silicate or carbon) [3]. At low gas densities, radiative association reactions and ion-molecule reactions may dominate in producing new molecules. In dense molecular clouds, dust grain surfaces and the ice layers condensed on the grains are important places for new molecules to form [4, 5]. However, the detailed reaction mechanisms have not been identified because the reaction rates in space are so slow that the observations can only provide a snapshot of the processes. The molecule formation processes usually need an external energy input, such as heat or irradiation, to overcome the reaction barriers. The source of radiant energy occurs in the form of UV photons, cosmic rays or X-rays.

For decades, scientists have been using numerous experimental methods to verify or refute hypotheses about chemical mechanisms in space. This thesis aims to use laboratory experimental methods to investigate photochemical effects which occur on astronomical ices and dust grains. We first focus on the study of $\mathrm{CO}_{2}$ ice, one of the most abundant ice components in the ISM, to explain the UV-induced effects during its formation, destruction and desorption. We also study the role of $\mathrm{SiO}_{2}$ dust grains in the formation of complex molecules. 


\subsection{The Interstellar Medium (ISM)}

The interstellar medium can be classified into three phases: cold neutral medium, warm neutral or ionized medium, and hot ionized medium [6]. In hot and warm ionized medium, the ionized species can be traced through UV absorption lines against background sources. The neutral atomic medium is dominated by atomic hydrogen, which can be traced by the $21 \mathrm{~cm}$ line [7].

The interstellar medium plays an important role in galaxy evolution, as it is the birth place of stars. Molecular clouds are usually rich in molecular components; therefore they are the active regions of star formation. $\mathrm{H}_{2}$ is the dominant species in molecular clouds; $\mathrm{CO}, \mathrm{CO}_{2}$, and $\mathrm{H}_{2} \mathrm{O}$ are also an abundant component. Other molecules, including simple organic molecules, are also observed in molecular clouds [8]. The evolution pathways of those molecules are the mysteries we try to solve here.

During the evolution of molecular clouds, the atoms and ions will have a higher probability to meet each other as the clouds become denser. The relatively high density of these molecular clouds can shield the destructive high energy photons and cosmic rays, therefore protect the newly formed species from being destroyed [9]. This provides shelter for the newly formed molecules. For example, it is observed that as the visual extinction grows, the molecular $\mathrm{H}_{2}$ becomes more dominant over atomic $\mathrm{H}$ [1].

As the clouds become denser and more molecular species formed, the cooling efficiency gets higher because more radiation modes are available, causing a decreasing of cloud temperature. Dust grains can also radiate the energy they absorbed, and contribute to the cooling process [1]. As a consequence of cooling, the gravitational force becomes more dominant over the thermal pressure, a new protostar can then form in the center of a cloud. 
In this thesis we are mainly concerned chemical reactions in conditions relevant to dense molecular clouds where star formation occurs. The relatively higher density also allows chemical reactions to take place more efficiently, to form more complex molecules.

\subsection{Chemistry in the Interstellar Medium}

Studies on the chemistry in the interstellar medium started in the 1940s when $\mathrm{CH}$ and $\mathrm{CH}^{+}$were found in the ISM [10]. But only a few reactions were investigated because of the lack of knowledge of interstellar molecules. The development of radio astronomy in the 1960s provides a new powerful tool to discover many new molecules in the ISM, and hence led to a fast development of astrochemistry. Until now, around 180 molecules have been identified in space $[8,11]$, yet many observed lines have not been attributed to specific species.

The molecular emission lines are important in the detection and identification of molecules in the ISM. There are mainly three types of molecular emission: electronic emission, vibrational emission, and rotational. However, in dense molecular clouds, the temperature is usually too low to excite the molecules electronically or vibrationally. Therefore, most interstellar molecules are detected by their rotational emission line features through observations in radio bands.

The reaction conditions in the molecular clouds include low temperatures (typically $<100 \mathrm{~K})$, low densities $\left(\sim 1-10^{4}\right.$ molecules $\left.\mathrm{cm}^{-3}\right)$, and strong radiation fields (UV photons, cosmic rays, etc.). Therefore the chemical reactions occurring in space are quite different from those on earth. Because of the low temperature, reactions with high activation barriers are not likely to occur. Thus, reactions involving radicals and charged particles are favored because many of them have little or no activation barrier. 
The low density of the ISM also favors the formation of radicals and charged particles, because the collision rate between them is low. Another outcome of the low density environment is that the reaction time scales may be much longer than that on Earth. It may take thousands to millions of years to produce a detectable amount of molecules.

UV photons are one of the major forms of radiation in the ISM. Among the UV photons, the dominant one is the Lyman- $\alpha(10.2 \mathrm{eV})$ photons, which is emitted from excited $\mathrm{H}$ atoms produced by proton capture of electrons. Since $\mathrm{H}$ is the most abundant species in space, the Lyman- $\alpha$ radiation is almost ubiquitous. The flux of UV photons is usually high along the edge of molecular clouds $\left(10^{8}\right.$ photons $\left.\mathrm{cm}^{-2} \mathrm{~s}^{-1}\right)$ [12], and is low in the center of dense clouds $\left(10^{3}\right.$ photons $\mathrm{cm}^{-2}$ $\mathrm{s}^{-1}$ ) because of the shielding effect $[13,14]$.

\begin{tabular}{|c|c|c|}
\hline Species & $\sigma_{\text {pd }}\left(\mathbf{c m}^{2}\right)$ & Reference \\
\hline $\mathrm{H}_{2} \mathrm{O}(\mathrm{g})$ & $1.2 \times 10^{-17}$ & {$[16]$} \\
\hline $\mathrm{H}_{2} \mathrm{O}$ (ice) & $9 \times 10^{-18}$ & {$[17]$} \\
\hline $\mathrm{OH}(\mathrm{g})$ & $1.8 \times 10^{-18}$ & {$[18]$} \\
\hline $\mathrm{CO}_{2}$ (g) & $6.1 \times 10^{-20}$ & {$[19]$} \\
\hline $\mathrm{CO}_{2}$ (ice) & $9 \times 10^{-18}$ & {$[20]$} \\
\hline $\mathrm{N}_{2} \mathrm{O}(\mathrm{a})$ & $6.9 \times 10^{-20}$ & {$[21]$} \\
\hline $\mathrm{CH}_{3} \mathrm{OH}(\mathrm{g})$ & $1.4 \times 10^{-17}$ & {$[22]$} \\
\hline $\mathrm{CH}_{4}(\mathrm{~g})$ & $1.8 \times 10^{-17}$ & {$[23]$} \\
\hline
\end{tabular}

Table 1.1: List of Lyman- $\alpha$ photodissociation cross sections for some molecules related to studied in this thesis.

Upon irradiation of Lyman- $\alpha$ photons, most molecules can dissociate except a few molecules with very strong chemical bonds (such as $\mathrm{CO}, \mathrm{N}_{2}$ and $\mathrm{CN}$ ). The photodissociation cross section for molecules is an important parameter in both 
experimental measurements and theoretical modeling. A comprehensive summary of cross sections for key molecules has been made by van Dishoeck et al. [15]. Some of the cross sections which are related to the study in this thesis are listed in Table 1.1.

\subsection{The Ices and Dust Grains in the ISM}

Interstellar dust grains are typically small particles of micrometer size, and they are an important part of the ISM. Their components are usually silicate or carbonaceous material. The grains may absorb and scatter light, and control the spectral energy distribution of the ISM [1]. They may also lock up some fraction of heavy elements, resulting in a depletion of element abundances [1]. Observations find that many heavy elements are significantly depleted in the ISM compared to some cosmic abundances (such as in the Sun), indicating that these elements may have condensed into solid dust grains [24].

Because of the development of radio astronomy, more and more species have been found in the ISM. However their formation mechanisms are still not clear, especially for the complex molecules. For many of them, simple gas phase formation mechanisms such as two-body collision reactions are not sufficient to explain the observed abundances, suggesting a more efficient pathway must exist for molecular formation. For example, the $\mathrm{H}_{2}$ abundance in many molecular clouds is much higher than that predicted by gas phase models. Therefore, grain surface reactions and solid phase reactions are generally considered in the explanation of interstellar molecule formation [25].

It is believed that grain surface reactions can facilitate the molecule formation processes and hence produce the observed abundances of many complex molecules[26]. Typically, molecules firstly accrete on the grain surface at low temperatures. Although most heavy molecules except $\mathrm{H}$ and $\mathrm{H}_{2}$ are not very mobile in cold conditions, they can absorb irradiations, such as photons and 
cosmic rays, and break into radicals and reactive fragments. Then during the warm up phase of the cloud core, these fragments can become mobile and react with each other to form the complex species.

Besides gas phase molecules, many solid phase species are also detected in the ice mantles of dust grains. The first ice detected in the ISM was water, as it is the most abundant ice component in many molecular clouds [27]. $\mathrm{CO}$ and $\mathrm{CO}_{2}$ are also common components of ices and their fraction can be as much as $30 \%$ relative to water $[28,29]$. Other ices like $\mathrm{CH}_{3} \mathrm{OH}, \mathrm{NH}_{3}, \mathrm{CH}_{4}$ and $\mathrm{CN}$ are also widely detected [30,31]. The molecules which eventually produce ice species may first form in the gas phase and then accrete on the grain surface, but some of them cannot be solely attributed to gas phase formation and a grain-surface formation route should be considered. For example, the observed $\mathrm{CO}_{2}$ ice abundance is much higher than it in the gas phase, suggesting the $\mathrm{CO}_{2}$ containing ice is formed through solid phase reactions [32].

In the ISM, most ices are mixed, though a few pure ices are also observed $[28,33]$. The mixed ices provide a rich environment for chemical reactions to occur, but also provide challenges for people who want to study them. Ice infrared spectroscopy is a key to understanding the components and molecular environment in the ices, as molecules in ice form exhibit different infrared spectral features compared to those in the gas phase. The environment of molecules in the ices can also affect their infrared spectra, like changing of peak shapes or shifting of peak frequencies. For example, crystalline $\mathrm{CO}_{2}$ ice exhibits a double peak $v_{2}$ band at $15 \mu \mathrm{m}$, while amorphous or mixed $\mathrm{CO}_{2}$ ices show only a single band $[34,35]$. When $\mathrm{CO}_{2}$ ice is mixed with $\mathrm{H}_{2} \mathrm{O}$, a red shift of the $\mathrm{CO}_{2} \mathrm{v}_{3}$ band is also observed, because the molecular dipole moments can be affected by the neighboring molecules $[36,37]$. These features provide us useful information to study the ice components and ice dynamics. 
Photodissociation and photodesorption are important processes that occur when the ices interact with UV photons. The absorbed photon energy can dissociate or ionize the molecules to make reactive species which then form new species. It also causes a desorption of molecules into gas phase, and provides an explanation to the observed gas phase abundance of several molecules which cannot be attributed to thermal desorption only [38-41].

In this thesis we focus on the studies of photon-induced physical and chemical processes which occur in the ices, using infrared spectroscopy and mass spectrometry methods. We also investigated the role of grain surface reactions in the formation of new molecules.

\subsection{Astrochemistry in the Laboratory}

Laboratory astrochemistry plays an important role in understanding the physical and chemical processes in the interstellar medium. First, it provides an interpretation of the observed spectra. The laboratory spectroscopic measurements can help us to identify the species observed, and also may reveal the physical conditions of those species. Second, the laboratory studies will give much more detailed information about the reactions. Many modern techniques can closely monitor the reaction processes in situ and may observe the intermediate products. This will help us to establish a complete picture of the molecule evolution in the ISM. Because the actual reactions in space occur at a much lower rate - usually take thousands to millions of years, the laboratory work can help us to understand the whole picture in a few hours, by accelerating the reaction processes with higher radiation flux or heating rates.

In the ISM, the surfaces of dust grains are crucial places for the production of new molecules, and it is believed that grain surface chemistry is involved in producing the observed abundance of many complex molecules. The grain surface reaction is more efficient than the gas phase reaction because the grain 
serves as a third body to absorb excess energy released in the reaction and thus the reaction probability is higher. In a typical grain surface reaction, gas molecules adsorb on the grain surface, diffuse across the surface, react with another adsorbed molecule, and finally desorb from the grain surface. These elementary surface processes are the targets of modern surface science and can be studied in detail using experimental surface science methods. The study of surface processes can help us to understand many properties of grain surface reactions, such as the diffusion energy, desorption energy, reaction mechanisms, and many others.

\subsubsection{The Vacuum System}

To mimic the environment of the interstellar medium in the laboratory, we constructed a stainless steel vacuum chamber which is pumped by a turbo molecular pump and an ion pump, as shown in Figure 1.1. A pair of sample holders (Figure 1.2) made with Ni bars is connected to a rotatable cooling dewar, and is placed in the center of the chamber. The dewar can be cooled with liquid nitrogen $\left(\mathrm{I}-\mathrm{N}_{2}\right)$, and may be further cooled $(\sim 10 \mathrm{~K})$ by bubbling $\mathrm{He}$ gas through I$\mathrm{N}_{2}$ [42]. The sample (made with amorphous $\mathrm{SiO}_{2}$ ) or the sample support (made with $\mathrm{KBr}$ powder) are pressed onto a tungsten grid using a hydraulic laboratory press with 3000-5000 psi pressure. The tungsten grid $(0.005 \mathrm{~cm}$ thick) has 22 $\mu \mathrm{m} \times 22 \mu \mathrm{m}$ openings and passes about $60 \%$ of the incident IR radiation. The sample diameter is $0.7 \mathrm{~cm}$; therefore the sample area is $0.3 \mathrm{~cm}^{2}$. A K-type thermocouple is welded onto the tungsten grid in order to read the temperature of the sample. The accuracy of temperature measurement is $\pm 0.1 \mathrm{~K}$ relatively and $\pm 2 \mathrm{~K}$ absolutely. The lowest sample temperature achieved in experiments is $\sim 84$ $\mathrm{K}$ with I- $\mathrm{N}_{2}$ cooling only, and $\sim 72 \mathrm{~K}$ with the He bubbling technique. 


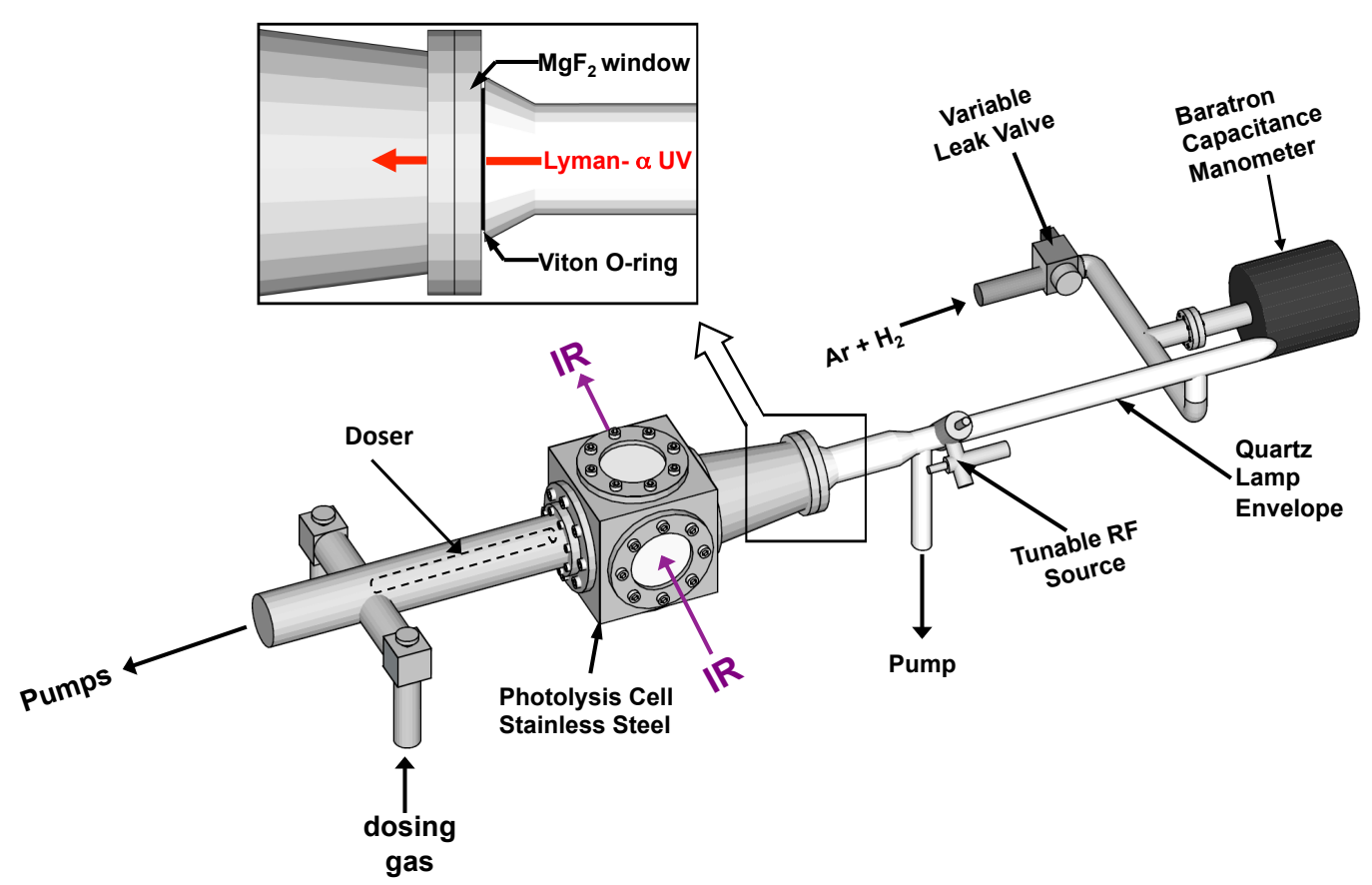

Figure 1.1: Schematic of the vacuum chamber and Lyman- $\alpha$ lamp.

Two $\mathrm{KBr}$ windows with differential pumping are mounted to the left and right sides of the chamber, to allow the infrared beam to pass through. The differential pumping prevents air leaking through the O-ring seals around the $\mathrm{KBr}$ windows. A doser made with a copper tube (0.25" diameter) is placed at the back side of the chamber. Gas phase molecules can be introduced into the chamber through the doser through a leak valve, which accurately $\left(10^{-6} \mathrm{ML} \mathrm{s}^{-1}\right)$ controls the dosing rate. The sample is placed with $45^{\circ}$ incidence to the Lyman- $\alpha$ light and the infrared beam (as shown in Figure 1.2), and it can be rotated to face the doser during the dosing process. 


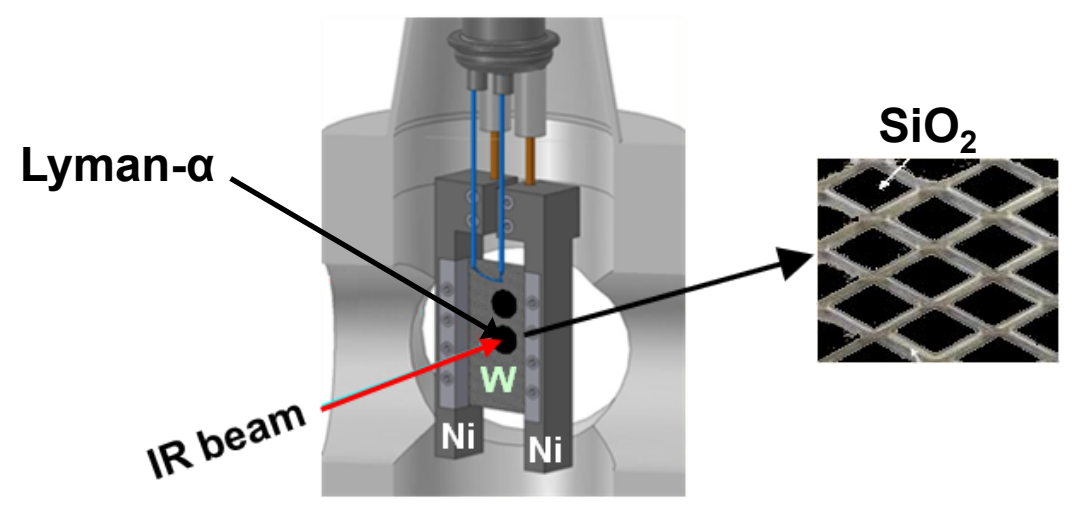

Figure 1.2: Schematic of the sample, grid, thermocouple and sample holders.

The base pressure of this vacuum chamber is about $1 \times 10^{-8}$ Torr. The main residual gas components in the chamber are $\mathrm{H}_{2}$ and $\mathrm{CO}$, with a trace amount of $\mathrm{H}_{2} \mathrm{O}, \mathrm{O}_{2}$ and $\mathrm{CO}_{2}$. The pumping speeds for $\mathrm{CO}$ and $\mathrm{CO}_{2}$ were measured experimentally. $\mathrm{CO}$ and $\mathrm{CO}_{2}$ gases were introduced in the chamber at a certain pressure. After quickly switching off the gas source, an exponential decay for pressure was recorded by the mass spectrometer. The time constants of the pressure curves give pumping speeds of $0.21 \mathrm{~L} \mathrm{~s}^{-1}$ for $\mathrm{CO}$, and $0.19 \mathrm{~L} \mathrm{~s}^{-1}$ for $\mathrm{CO}_{2}$. The accurate measurement of the pumping speeds for specific gases enables us to determine the absolute number of gas molecules evolved from ice surfaces in various experiments.

\subsubsection{Infrared Spectroscopy}

Infrared spectroscopy is a widely used tool in identifying the molecules in the ISM. Most molecules have absorption features in the infrared, except some diatomic molecules (such as $\mathrm{O}_{2}$ ) which do not have a permanent dipole moment. The infrared spectra can also tell the environment interactions around those molecules, by observing the peak frequency shift or the shape change of an absorbance band.

In our laboratory experiments, infrared spectroscopy is a powerful tool in the study of reaction processes. It is used to identify different molecules, monitor the 
product formation, and quantitatively measure the amount of each molecule using the integrated absorbance. The infrared spectrum of the sample is measured by a Bruker Tensor 27 Fourier Transform infrared spectrometer, with a liquid $\mathrm{N}_{2}$ cooled MCT detector. The measured wavenumbers usually range from 4000-500 $\mathrm{cm}^{-1}$, with a resolution of $2 \mathrm{~cm}^{-1}$. Each spectrum is averaged from 100-250 different scans, depending on the signal-to-noise ratio requirement of different measurements.

\subsubsection{Mass Spectrometry}

Mass spectrometry is a very sensitive and molecule-specific way to measure the reaction products in the desorption studies. It not only helps to identify the molecules which are hard to detect by infrared spectroscopy, but also measures the partial pressure of each gas component desorbing into the dynamically pumped vacuum system, which can be converted to the desorption rate using the known pumping speed.

A quadrupole mass spectrometer (QMS) is mounted to the bottom of the chamber in a second pumping line not shown in Figure 1.1. The ionization filament is about 2" away from the sample. The mass spectrometer (RGA 200) can work with a Faraday Cup or an electron multiplier with a gain up to $10^{6}$. Thus the detection limit of this mass spectrometer can reach as low as $10^{-13}$ Torr.

The QMS provides extraordinary sensitivity in our experimental measurements, which allow us to measure the desorption behavior of molecules from the outermost surface layer. For example, in our measurement of $\mathrm{CO}$ desorption from $\mathrm{CO}_{2}$ ice (Chapters 2 and 5), we have a sensitivity of the order of $10^{-7} \mathrm{ML} \mathrm{s}^{-1}$ or $\sim 10^{12}$ molecules $\mathrm{m}^{-2} \mathrm{~s}^{-1}$, whereas measurements of the photochemical rate of production of a molecule in the ice bulk, using the widelyapplied IR measurement method, have a sensitivity of only $\sim 10^{17}$ molecules $\mathrm{m}^{-2}$ $\mathrm{s}^{-1}$ if a strong vibrational mode is measured. 


\subsubsection{The Lyman- $\alpha$ Lamp}

Lyman- $\alpha$ light $(10.2 \mathrm{eV}, 121.6 \mathrm{~nm})$ is a ubiquitous type of UV irradiation in the Universe, because $\mathrm{H}$ is the most abundant element. In our laboratory, we produce Lyman- $\alpha$ light from a hydrogen discharge lamp. The lamp is made of a quartz tube, and is pumped by a mechanical pump, as shown in Figure 1.3. We use a $10 \% \mathrm{H}_{2} / 90 \%$ Ar pre-mixed gas flow through the lamp, with an incoming pressure of $1.000 \pm 0.010$ Torr. The use of constantly renewed $\mathrm{H}_{2} / \mathrm{Ar}$ gas leads to continuous replenishment of the gas and to sweeping gas impurities away. By using the $\mathrm{H}_{2} / \mathrm{Ar}$ mixed gas instead of pure $\mathrm{H}_{2}$ gas, we could eliminate the molecular excitation lines around 150-180 nm [43]. The lamp is excited by a microwave discharge power controller and the normal operation power is $60 \mathrm{~W}$. The lamp is sealed to a $\mathrm{MgF}_{2}$ window on the chamber by a Viton O-ring compressed between the lamp and the window. The cutoff for this window is at $1100 \AA$ [44], effectively removing possible Ar emission lines.

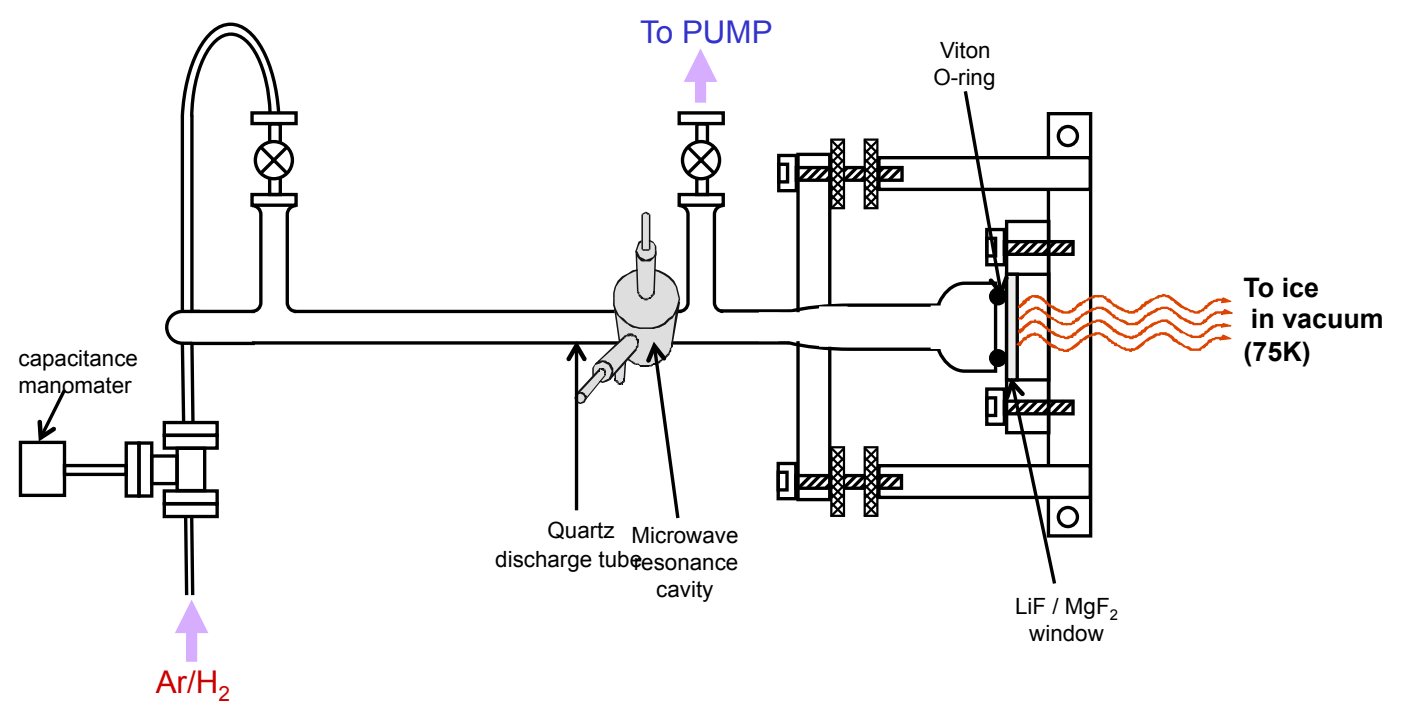

Figure 1.3: Structural design of the Lyman- $\alpha$ lamp.

The total flux of this lamp at normal operating condition is $(1.28 \pm 0.36) \times 10^{15}$ photons $\mathrm{cm}^{-2} \mathrm{~s}^{-1}$, measured by a chemical actinometer method using $\mathrm{N}_{2} \mathrm{O}$ gas 
[45]. The lamp flux is very stable over 500 hours operation in several years, and no defect or color center in the $\mathrm{MgF}_{2}$ window are observed. Two $\mathrm{N}_{2} \mathrm{O}$ actinometry measurements made 3 years apart showed that the lamp flux was stable to $10 \%$. An internal shutter allows the Lyman-a radiation to be turned on and off.

The Lyman- $\alpha$ light flux at the sample will be lower than measured near the $\mathrm{MgF}_{2}$ window using $\mathrm{N}_{2} \mathrm{O}$ actinometry. A moveable thermopile with a high angular acceptance angle was placed inside the cell at the sample position shown to measure the spatial distribution of radiation emitted by Lyman- $\alpha$ lamp. Experiments were done in pure flowing $\mathrm{N}_{2}$ at 1 atm. An approximately $\cos ^{300} \theta$ empirical angular distribution was measured in the vertical plane, and the light distribution in the horizontal plane was similar. The reflected radiation enhances lamp intensity at the sample. The empirical $\cos ^{300} \theta$ function includes significant contributions from inner reflections in the apparatus. We assume that the Lyman$\alpha$ radiation exhibits a similar angular distribution to the power distribution measured by the thermopile. As shown in Figure 1.4, the distribution of radiation is extremely forward focused. This directionality is due to light focusing in both the lamp and inside the cell as a result of reflections at interior surfaces. The sample if normal to the Lyman- $\alpha$ source would receive $1.16 \times 10^{14}$ photons $\mathrm{s}^{-1}$ within the angular distribution measured. Since for all photochemical measurements, the sample is mounted at $45^{\circ}$ to both the Lyman-a source and the IR beam, the corrected Lyman- $\alpha$ intensity received by the sample is $0.82 \times 10^{14}$ photons $\mathrm{s}^{-1}$. The flux of Lyman- $\alpha$ radiation at the $0.3 \mathrm{~cm}^{2}$ sample is therefore $2.7 \times 10^{14}$ photons $\mathrm{cm}^{-2} \mathrm{~s}^{-1}$. 


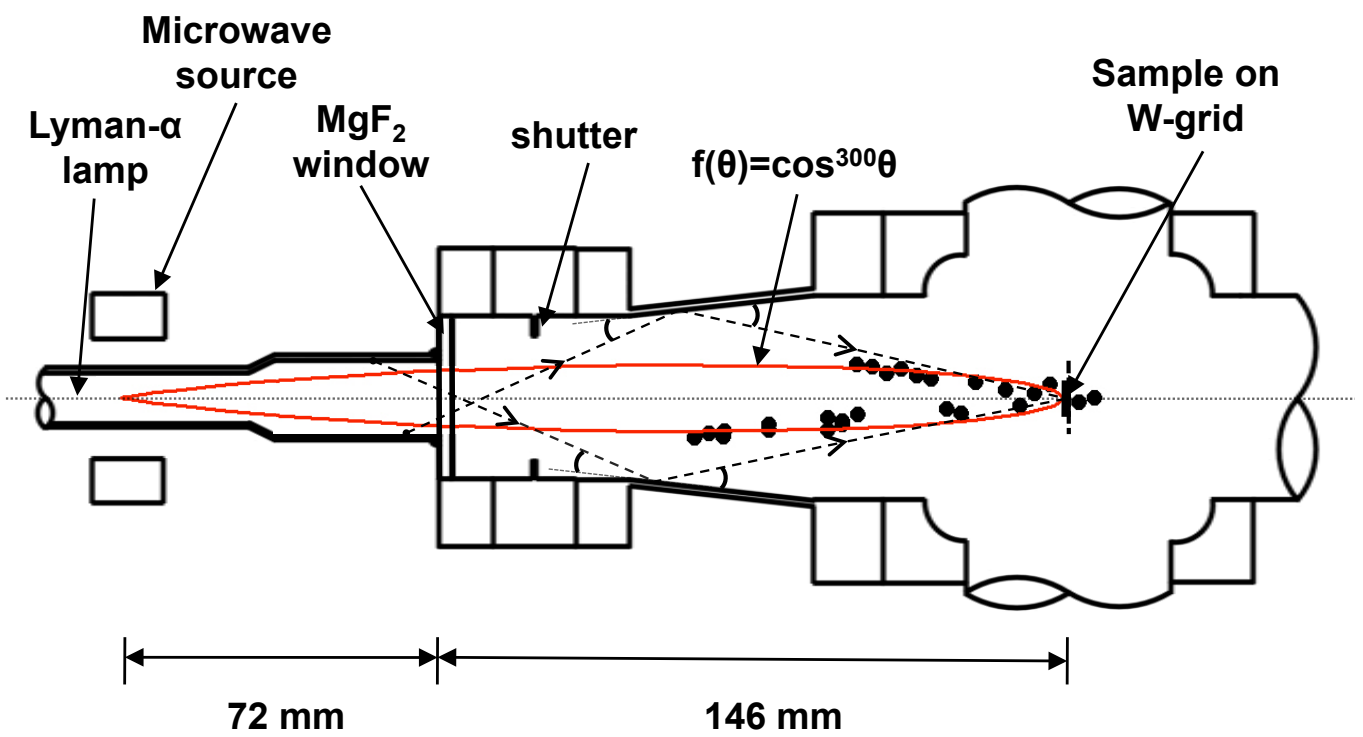

Figure 1.4: Measured distribution of emitted power from Lyman- $\alpha$ source using a thermopile. The distribution shown by the black points is very strongly peaked in the forward direction and estimated to be $\cos ^{300} \theta$, as shown by the red angular distribution.

\subsection{This Thesis}

This thesis describes our laboratory studies of photochemical effects and reactions on both astronomical ices and dust grains. Chapters 2-5 show a comprehensive study of the life cycle of $\mathrm{CO}_{2}$ ice, from its formation pathways to its photodissociation and photodesorption mechanisms; Chapters 6-7 show the photo-induced surface reactions of $\mathrm{N}_{2} \mathrm{O}$ molecules adsorbed on $\mathrm{SiO}_{2}$ grain surfaces. The Appendix includes a study of physical adsorption on $\mathrm{C}_{60}$ film as well as details of our measurement methods. 


\subsubsection{Photochemical Effects on the Life Cycle of Interstellar $\mathrm{CO}_{2}$ Ices}

$\mathrm{CO}_{2}$ ice was first detected in the ISM in 1989 by d'Hendecourt \& de Muizon [46]. Since then, it has been observed in numerous surveys of different interstellar objects $[29,30,37]$. One of the key findings is that the abundance of $\mathrm{CO}_{2}$ in the ice form is much higher than that in the gas phase, suggesting a solid phase formation route to produce $\mathrm{CO}_{2}$ ice [32]. The desorption and destruction processes of $\mathrm{CO}_{2}$ ice is also important to explain the observed gas phase $\mathrm{CO}_{2}$ abundance and the formation of other molecules. In this thesis, we focus on the study of photochemical effects on the formation, dissociation and desorption mechanisms of $\mathrm{CO}_{2}$ ice.

Experimental studies have proposed many different reaction pathways. Loeffler et al. [47] irradiated $\mathrm{CO}$ ices with $200 \mathrm{keV}$ protons and observed $\mathrm{CO}_{2}$ formation by $\mathrm{CO}$ reacting with an excited $\mathrm{CO}$ molecule. They also compared the amount of $\mathrm{CO}_{2}$ produced with that generated using Lyman-a photons. Jamieson et al. [48] irradiated $\mathrm{CO}$ ices with energetic electrons and observed the formation of carbon dioxide, as well as other carbon oxide species.

The formation of $\mathrm{CO}_{2}$ has also been found by $\mathrm{CO}$ reacting with atomic oxygen. Roser et al. [49] studied the reactivity of $\mathrm{CO}$ toward $\mathrm{O}$ atoms and detected the formation of $\mathrm{CO}_{2}$ using temperature programmed desorption. Madzunkov et al. [50] also studied the production of $\mathrm{CO}_{2}$ by depositing an $\mathrm{O}$ beam onto a CO-ice surface. However, several theoretical investigations show that the $\mathrm{CO}+\mathrm{O}$ pathway has a high activation barrier [51-53], and it is generally believed that this reaction is not efficient in the ISM. Mennella et al. [54] produced $\mathrm{CO}$ and $\mathrm{CO}_{2}$ molecules from water ice covered carbon grains and exposed to energetic particles and Lyman-a photons. The results they obtained are in good agreement with observation. 
Reaction pathways associated with the formation of $\mathrm{CO}_{2}$ from $\mathrm{CO}$ and $\mathrm{OH}$ radicals have been studied both experimentally and theoretically. Gas-phase reaction between dilute $\mathrm{CO}$ and $\mathrm{OH}$ radicals has been widely studied since this reaction is important in the atmosphere [55, 56]. Oba et al. [57] and Zins et al. [58] studied this reaction in solid phase using non-energetic $\mathrm{OH}$ radicals, because $\mathrm{OH}$ radicals are likely to be present in their ground state, especially in dense molecular clouds. The reactivity of $\mathrm{CO}$ and $\mathrm{OH}$ radicals may play a crucial role in the formation of interstellar $\mathrm{CO}_{2}$, as suggested by Ruffle \& Herbst [53] based on computational modeling.

It should be noted that all of the previous experimental studies concerned solid phase CO reactions. However, in the very low temperature environment in the ISM, both $\mathrm{CO}$ and $\mathrm{OH}$ are mostly immobile, due to their relatively high diffusion barrier [59]. Therefore, a different reaction mechanism is desired. In Chapter 2, we propose a new Eley-Rideal type surface reaction mechanism, which is kinetically different from previously studied solid phase reactions.

The Eley-Rideal mechanism describes a process where an incoming species from the gas phase directly collides with a surface-bound species to produce a product. It was first proposed as the mechanism of a catalysis reaction on a metal surface by Eley and Rideal on 1930s [60-62]. In Chapter 2 we show the experimental study of $\mathrm{CO}$ gas molecules reacting with photochemicallygenerated surface $\mathrm{OH}$ species on water ice surfaces. This study demonstrates a new mechanism to form $\mathrm{CO}_{2}$ in the ISM, which may contribute to the modeling of $\mathrm{CO}$ and $\mathrm{CO}_{2}$ abundances in space.

$\mathrm{CO}_{2}$ (ice) formed on grain surfaces may be subjected to strong UV irradiation in the ISM. UV photons can break the $\mathrm{C}-\mathrm{O}$ bond and form $\mathrm{CO}$ and $\mathrm{O}$, and further form $\mathrm{CO}_{3}$ and $\mathrm{O}_{3}[13,63,64]$. In Chapter 3 we thoroughly studied the $\mathrm{CO}_{2}$ (ice) photodissociation process and we focus on the kinetic isotope effect which is seen during the photodissociation. The structure and isotopic composition of 
molecules detected in interstellar space provide a method for categorizing the origin of the molecules found. The study of the relative abundance of UVprocessed isotopomers is important for us to understand the stellar nucleosynthesis and the chemical evolution of molecules in interstellar space and in the solar system [65-68].

We report that the Lyman-a photodissociation of the $\mathrm{CO}_{2}$ molecule in $\mathrm{CO}_{2}$ (ice) occurs with a significantly larger $\mathrm{C}$-O bond dissociation rate for the ${ }^{12} \mathrm{C}$ isotopomer of $\mathrm{CO}_{2}$ compared to ${ }^{13} \mathrm{CO}_{2}$. This kinetic isotope effect $(\mathrm{KIE})$ is postulated to occur as a result of isotopic differences in the probability of dissociating the $\mathrm{C}-\mathrm{O}$ bond in electronically-excited $\mathrm{CO}_{2}{ }^{*}$ molecules when in close contact with the ice matrix. The ice matrix supplies electronic quenching routes for the excited molecule. This leads to a mass-dependent rate of photodissociation favoring ${ }^{12} \mathrm{CO}_{2}$ over ${ }^{13} \mathrm{CO}_{2}$. Electronic quenching of the two isotopomers of the excited state of $\mathrm{CO}_{2}$ occurs with different probabilities by energy transfer to the $\mathrm{CO}_{2}$ matrix as the electronically-excited $\mathrm{CO}_{2}{ }^{*}$ molecule begins to dissociate along the upper-state repulsive potential curve associated with the excited state. Such effects are well known for molecules adsorbed on surfaces and may be understood in ices in the same mechanistic framework as developed for surfaces.

The desorption of molecules from ices in interstellar space to produce the gas molecules is also an important process in the molecular evolution. Two processes which drive desorption from ices are thermal desorption and radiationdriven desorption (photodesorption). In Chapters 4 and 5 we mainly deal with the photodesorption of $\mathrm{CO}_{2}$ ice induced by Lyman- $\alpha$ radiation.

Chapter 4 shows the dependence of photodesorption efficiency on the ice components. We demonstrate how within the $\mathrm{CO}_{2}$ ice matrix, the matrixmediated conversion of electronically-excited $\mathrm{CO}_{2}$ back to highly vibrationallyexcited $\mathrm{CO}_{2}$ subsequently results in two main kinetic channels: (1). The 
vibrational relaxation of the excited molecule as it is retained in the ice matrix, and (2). The escape of the vibrationally-excited $\mathrm{CO}_{2}$ molecule by desorption from the ice. By varying the vibrational coupling between the excited molecule and the ice matrix, we find that the efficiency of $\mathrm{CO}_{2}$ photodesorption is strongly correlated inversely to the efficiency of $\mathrm{CO}_{2}$ vibrational relaxation in the ice.

Chapter 5 shows how the interaction between high energy photons and $\mathrm{aCO}_{2}$ ice lattice affects the desorption efficiency. The destructive interaction between extreme UV radiation and ices has been shown to greatly enhance the rate of photodesorption; it is believed that irradiation with photons or electrons will cause photosensitive ices to become more porous and to exhibit weaker intermolecular bonding as increasing crystal disorder is produced. Radiation-induced changes in the ice structure influence the photodesorption rate of molecules as well as the trapping of photoproducts.

In studies of Lyman- $\alpha$ induced $\mathrm{CO}_{2}$ photodesorption, Öberg et al. [40] observed a delayed photodesorption of $\mathrm{CO}$ from crystalline $\mathrm{CO}_{2}$ (ice) at $40-60 \mathrm{~K}$, but this delay did not occur for amorphous ice deposited at $18 \mathrm{~K}$. They also compared the photodesorption yield of thermally-annealed $\mathrm{CO}_{2}$ (ice) with unannealed ice at $18 \mathrm{~K}$, finding that the former yield is $\sim 0.4$ times that of the latter. This indicates that the $\mathrm{CO}$ photodesorption yield is dependent on the ice structure and that annealing of amorphous $\mathrm{CO}_{2}$ (ice) reduces the $\mathrm{CO}$ desorption rate. Similar observations were made by Bahr et al. [69]

In Chapter 5 we show that large structural changes in the $\mathrm{CO}_{2}$ (ice) occur during Lyman-a induced photochemistry. In a $50 \mathrm{~nm} \mathrm{CO}$ ice film at $75 \mathrm{~K}$, radiation damage increases the $\mathrm{CO}$ photodesorption rate by $\sim 90$ fold and the $\mathrm{CO}_{2}$ photodesorption rate by $\sim 8$ fold. These effects are only observed readily using a type of photodesorption measurement particularly sensitive to the kinetics of desorption from the ice surface. We demonstrate that radiation damage, causing crystal structure disorder to ices, produces new more efficient molecular 
transport pathways which are accessed by photoexcitation processes. Such radiation damage effects will therefore have to be considered in models concerned with the gas phase composition of regions of the ISM where photodesorption from ices is a major factor in supplying gas phase products.

\subsubsection{Surface Reactions on Silicate Dust Grains}

On grain surfaces in the ISM, it is generally accepted that the recombination of molecules or radicals will occur preferentially on surfaces (compared to the gas phase) to produce more complex molecules. Experimental evidence for enhanced combination kinetics for $\mathrm{H}$ atoms near $10 \mathrm{~K}$ on silicate and graphite surfaces has been obtained by Vidali et al. [70-72] and surface combination of $\mathrm{H}$ atoms is deemed to be responsible for the high $\mathrm{H}_{2}$ abundance found in the interstellar medium where $\mathrm{H}_{2}$ destruction by cosmic rays in the gas phase competes with $\mathrm{H}_{2}$ formation on surfaces. Fragment combination processes in the dilute gas phase are expected to be very slow due to the need for a third body to carry off excess energy generated when bonds form between fragment species to produce more complex combination products.

Despite the belief that surfaces promote molecular fragment combination, there are no experimental reports quantitatively comparing molecular fragment combination rates on surfaces to rates in the gas phase for any species other than atomic $\mathrm{H}$. The experiment described in Chapter 6 compares a photochemical process in the gas phase to the same process within the pores of a silica powder containing adsorbed molecules, where surface effects will be highly amplified compared to those in the gas phase. We report that more complex products from photochemically-generated radical fragment combination reactions are selectively enhanced by the proximity of the silica surface.

By comparing the rate of the association reactions on a silica surface to those in the gas phase, it has been found that the surface kinetically favors the 
association products by a factor of $\sim 4$ and $\sim 20$ respectively for $\mathrm{NO}_{2}$ and $\mathrm{N}_{2} \mathrm{O}_{4}$ products made from the photodecomposition of adsorbed $\mathrm{N}_{2} \mathrm{O}$ on $\mathrm{SiO}_{2}$. This indicates the importance of the surface, acting as a third-body, in mediating associative recombination.

In addition to the general third-body effect on enhancing association reactions on surfaces, the reaction rate may be also affected by the functional groups present on the surface. The association reaction can be negatively influenced by a cage effect, in which functional groups on the surface impede radical-radical reactions by molecular shielding; Alternatively, the surface may promote a negative effect on reaction kinetics by quenching the primary photochemical reaction by the removal of electronic energy from the excited state. Functional groups, acting as a spacer layer, would be expected to remove or reduce such quenching effects. An indirect photochemical process may also occur and enhance the reaction rate by the production of unstable temporary negative ions due to electron transfer from the surface caused by electron-hole pair formation in the $\mathrm{SiO}_{2}$ under Lyman-a excitation.

In Chapter 7, the experiments are focused on the production of $\mathrm{N}_{2} \mathrm{O}$ photoproducts made from adsorbed $\mathrm{N}_{2} \mathrm{O}$ on $\mathrm{SiO}_{2}$ and chemically-modified $\mathrm{SiO}_{2}$ surfaces upon excitation with Lyman-a radiation at low temperatures. The experiment compares the rate of association product formation for Lyman-a induced $\mathrm{N}_{2} \mathrm{O}$ photodissociation on five types of $\mathrm{SiO}_{2}$ surfaces, either unfunctionalized bare $\mathrm{SiO}_{2}$ (called $\mathrm{Si}-\mathrm{O}-\mathrm{Si}$ ), or surfaces containing $\mathrm{Si}-\mathrm{OH}$ functionalities, $\mathrm{Si}-\mathrm{OCH}_{3}$ functionalities, $\mathrm{Si}-\mathrm{O}-\mathrm{SiCl}_{3}$ functionalities, or $\mathrm{SiO}_{2}$ surfaces containing hundreds of monolayers of amorphous $\mathrm{H}_{2} \mathrm{O}$ ice.

It is shown that four types of surface functionalization of the $\mathrm{SiO}_{2}$ surface cause $\mathrm{a} \sim 50 \%$ depletion in the rate of association product ( $\mathrm{NO}$ and $\mathrm{NO}_{2}$ ) formation at $75 \mathrm{~K}$ compared to reaction on the unfunctionalized $\mathrm{SiO}_{2}$ surface. The studies are inconsistent with the notion that preferential quenching of 
electronically excited surface species occurs on the unfunctionalized, bare $\mathrm{SiO}_{2}$ surface.

\subsection{Reference}

1. Tielens, A.G.G.M., The Physics and Chemistry of the Interstellar Medium, 2005, Cambridge, UK: Cambridge University Press.

2. Lequeux, J., E. Falgarone, and C. Ryter, The Interstellar Medium, 2005:

Astronomy and Astrophysics Library (Springer).

3. Tielens, A.G.G.M. and W. Hagen, Model calculations of the molecular composition of interstellar grain mantles. Astron. Astrophys., 1982. 114: p. 245.

4. Ruffle, D.P. and E. Herbst, New models of interstellar gas-grain chemistry - II. Surface photochemistry in quiescent cores. Monthly Notices of the Royal Astronomical Society, 2001. 322: p. 770.

5. Ruffle, D.P. and E. Herbst, New models of interstellar gas-grain chemistry - I. Surface diffusion rates. Monthly Notices of the Royal Astronomical Society, 2000. 319: p. 837.

6. Snow, T.P. and B.J. McCall, Annu. Rev. Astron. Astrophys., 2006. 44: p. 367.

7. Muller, C.A. and J.H. Oort, Nature, 1951. 168: p. 357.

8. Woon, D.E., Interstellar and Circumstellar Molecules. http://www.astrochymist.org/astrochymist ism.html.

9. $\quad$ Clayton, R.N., Nature, 2002. 415: p. 860.

10. Swings, P., Astrophys. J., 1942. 95: p. 270.

11. Molecules in Space, Cologne Database. Available from: https://www.astro.unikoeln.de/cdms/molecules.

12. Mathis, J.S., P.G. Mezger, and N. Panagia, Astron. Astrophys., 1983. 128: p. 212.

13. Gerakines, P.A., W.A. Schutte, and P. Ehrenfreund, Ultraviolet processing of interstellar ice analogs. I. Pure ices. Astron. Astrophys., 1996. 312: p. 289.

14. Sternberg, A., A. Dalgarno, and S. Lepp, Cosmic-ray-induced photodestruction of interstellar molecules in dense clouds. Astrophys. J., 1987. 320: p. 676. 
15. van Dishoeck, E.F., B. Jonkheida, and M.C. van Hemert, Photoprocesses in protoplanetary disks. Faraday Discussions, 2006. 133: p. 231.

16. Lee, L.C. and M. Suto, Chem. Phys., 1986. 110: p. 161.

17. Watanabe, N., T. Horii, and A. Kouchi, Measurements of $D_{2}$ yields from amorphous D2O ice by ultraviolet irradiation at $12 \mathrm{~K}$. Astrophys. J., 2000. 541(2): p. 772.

18. van Dishoeck, E.F. and A. Dalgarno, Icarus, 1984. 59: p. 305.

19. Yoshino, K., et al., Absorption cross section measurements of carbon dioxide in the wavelength region 118.7-175.5 $\mathrm{nm}$ and the temperature dependence. J. Quant. Spectrosc. Radiat. Transfer, 1996. 55: p. 53.

20. Yuan, C. and J.T. Yates, Jr., Isotope Effect in the Photochemical Decomposition of $\mathrm{CO}_{2}$ (ice) by Lyman-a Radiation. J. Chem. Phys., 2013. 138: p. 154302.

21. Rajappan, M., C. Yuan, and J.T. Yates, Jr., Lyman-a driven molecule formation on $\mathrm{SiO}_{2}$ surfaces - connection to astrochemistry on dust grains in the interstellar medium. J. Chem. Phys., 2011. 134: p. 064315.

22. Nee, J.B., M. Suto, and L.C. Lee, Chem. Phys., 1985. 98: p. 147.

23. Lee, L.C. and C.C. Chiang, Chem. Phys., 1983. 78: p. 688.

24. Snow, T.P. and A.N. Witt, Astrophys. J., 1996. 468: p. L65.

25. Hasegawa, T.I., E. Herbst, and C.M. Leung, Models of gas-grain chemistry in dense interstellar clouds with complex organic molecules. Astrophysical Journal Supplement Series 1992. 82: p. 167.

26. Hollenback, D., M.W. Werner, and E.E. Salpeter, Astrophys. J., 1971. 163: p. 165.

27. Gillett, F.C. and W.J. Forrest, Astrophys. J., 1973. 179: p. 483.

28. Pontoppidan, K.M., et al., A 3-5 $\mu \mathrm{m}$ VLT spectroscopic survey of embedded young low mass stars: I - Structure of the CO ice Astron. Astrophys., 2003. 408: p. 981.

29. Pontoppidan, K.M., et al., The c2d Spitzer Spectroscopic Survey of Ices Around Low-Mass Young Stellar Objects. II. CO . Astrophys. J., 2008. 678: p. 1005.

30. Öberg, K.I., et al., The Spitzer Ice Legacy: Ice Evolution from Cores to Protostars. Astrophys. J., 2011. 740: p. 109.

31. Gibb, E.L., et al., Interstellar Ice: The Infrared Space Observatory Legacy. Astrophysical Journal Supplement Series, 2004. 151: p. 35. 
32. van Dishoeck, E.F., et al., A search for interstellar gas-phase $\mathrm{CO}_{2}$. Gas: solid state abundance ratios. Astronomy and Astrophysics, 1996. 315: p. L349.

33. Dartois, E., et al., Carbon dioxide-methanol intermolecular complexes in interstellar grain mantles. Astron. Astrophys., 1999. 351: p. 1066.

34. Falk, M., Amorphous solid carbon dioxide. J. Chem. Phys., 1987. 86: p. 560.

35. Escribano, R.M., et al., Crystallization of $\mathrm{CO}_{2}$ ice and the absence of amorphous $\mathrm{CO}_{2}$ ice in space. Proceedings of the National Academy of Sciences of the United States of America, 2013. 110(32): p. 12899.

36. Sandford, S. and L. Allamandola, The physical and infrared spectral properties of $\mathrm{CO}_{2}$ in astrophysical ice analogs. Astrophys. J., 1990. 355: p. 357.

37. Gerakines, P.A., et al., Observations of Solid Carbon Dioxide in Molecular Clouds with the Infrared Space Observatory. Astrophys. J., 1999. 522: p. 357.

38. Öberg, K.I., et al., Photodesorption of CO ice. Astrophys. J., 2007. 662: p. L23.

39. Oberg, K.I., et al., Photodesorption of ices II: $\mathrm{H}_{2} \mathrm{O}$ and $\mathrm{D}_{2} \mathrm{O}$. Astrophys. J., 2009. 693: p. 1209.

40. Oberg, K.I., E.F. van Dishoeck, and H. Linnartz, Photodesorption of ices I: CO, $\mathrm{N}_{2}$, and $\mathrm{CO}_{2}$. Astron. Astrophys., 2009. 496: p. 281.

41. Westley, M.S., et al., Photodesorption from low-temperature water ice in interstellar and circumsolar grains. Nature, 1995. 373: p. 405.

42. Xu, J., H.J. Jansch, and J.T. Yates, Jr., Cryogenic trick for enhanced cooling using liquid nitrogen. J. Vac. Sci. Technol. A, 1993. 11: p. 726.

43. Okabe, H., J. Opt. Soc. Am. , 1964. 54: p. 478.

44. Duncanson, A. and R.W.H. Stevenson, Proc. Phys. Soc., London, 1958. 72: p. 1001.

45. Rajappan, M., et al., Photochemical Decomposition of $\mathrm{N}_{2} \mathrm{O}$ by Lyman-a Radiation: Scientific Basis for a Chemical Actinometer. J. Phys. Chem. A, 2010. 114: p. 3443.

46. d'Hendecourt, L.B. and M.J. de Muizon, Astron. Astrophys., 1989. 223: p. L5.

47. Loeffler, M.J., et al., Astron. Astrophys., 2005. 435: p. 587.

48. Jamieson, C.S., A.M. Mebel, and R.I. Kaiser, Astrophysical Journal Supplement Series, 2006. 163: p. 184.

49. Roser, J.E., et al., Astrophys. J., 2001. 555: p. L61.

50. Madzunkov, S., et al., Phys. Rev. A, 2006. 73: p. 020901. 
51. Goumans, T.P.M., A.U. Madeeha, and A.B. Wendy, Formation of $\mathrm{CO}_{2}$ on a carbonaceous surface: a quantum chemical study. Monthly Notices of the Royal Astronomical Society, 2008. 384: p. 1158.

52. Grim, R.J.A. and L.B. d'Hendecourt, Astron. Astrophys., 1986. 167: p. 161.

53. Ruffle, D. and E. Herbst, New models of interstellar gas-grain chemistry, III. Solid $\mathrm{CO}_{2}$. Monthly Notices of the Royal Astronomical Society, 2001. 324: p. 1054.

54. Mennella, V., M.E. Palumbo, and G.A. Baratta, Astrophys. J., 2004. 615: p. 1073.

55. Lester, M.I., et al., J. Chem. Phys., 2000. 113: p. 9889.

56. Li, J., et al., Quantum and quasi-classical dynamics of the $\mathrm{OH}+\mathrm{CO} \rightarrow \mathrm{H}+\mathrm{CO}_{2}$ reaction on a new permutationally invariant neural network potential energy surface. J. Chem. Phys., 2014. 140: p. 044327.

57. Oba, Y., et al., Experimental Study of $\mathrm{CO}_{2}$ Formation by Surface Reactions of Non-Energetic OH Radicals With CO Molecules. Astrophys. J., 2010. 712: p. L174.

58. Zins, E.-L., P.R. Joshi, and L. Krim, Reactivity Between a CO-Containing Ice and Non-Energetic OH Radicals. Astrophys. J., 2011. 738: p. 175.

59. Karssemeijer, L.J., et al., Dynamics of CO In Amorphous Water-Ice Environments. Astrophys. J., 2014. 781: p. 16.

60. Rideal, E.K., Proc. Cambridge Phil. Soc, 1938. 35: p. 130.

61. Eley, D.D., Adv. Catal. , 1948. 1: p. 157.

62. Eley, D.D. and E.K. Rideal, Nature, 1940. 146: p. 401.

63. Bennett, C.J., et al., Untangling the formation of the cyclic carbon trioxide isomer in low temperature carbon dioxide ices. Phys. Chem. Chem. Phys., 2004. 6: p. 735.

64. Moll, N.G., D.R. Clutter, and W.E. Thompson, Carbon trioxide: its production, infrared spectrum, and structure studied in a matrix of solid $\mathrm{CO}_{2}$. J. Chem. Phys., 1966. 45: p. 4469.

65. Rubin, R.H., et al., $12 \mathrm{C} / 13 \mathrm{C}$ ratio in planetary nebulae from the iue archives. Astrophys. J., 2004. 605: p. 784.

66. Vanysek, V. and J. Rahe, The ${ }^{12} \mathrm{C} /{ }^{13} \mathrm{C}$ Isotope Ratio in Comets, Stars and Interstellar Matter. Earth, Moon, and Planets, 1978. 18: p. 441.

67. Wyckoff, S., et al., The C-12/C-13 abundance ratio in Comet Halley. Astrophys. J., 1989. 339: p. 488. 
68. Hartogh, P., et al., Ocean-like water in the Jupiter-family comet 103P/Hartley 2. Nature, 2011. 478: p. 218.

69. Bahr, D.A. and R.A. Baragiola, Photodesorption of solid $\mathrm{CO}_{2}$ by Lya. Astrophys. J., 2012. 761: p. 36.

70. Pirronello, V., et al., Measurement of Molecular Hydrogen Formation on Carbonaceous Grains. Astron. Astrophys. , 1999. 344: p. 681.

71. Pirronello, V., et al., Laboratory Synthesis of Molecular Hydrogen on Surfaces of Astrophysical Interest". Astrophys. J., 1997. 475: p. L69.

72. Vidali, G., et al., Catalytic Activity of Interstellar Grains: Formation of Molecular Hydrogen on Amorphous Silicates. Adv. Space. Res. , 2009. 43: p. 1291. 


\section{Chapter 2: A New Source of $\mathrm{CO} 2$ in the Universe: A Photoactivated Eley-Rideal Surface Reaction on Ices}

Modified from manuscript submitted.

\subsection{Introduction}

The high abundance of $\mathrm{CO}_{2}$ in ices in the interstellar medium (ISM) greatly exceeds that in the gas phase[1]. This suggests that a solid phase $\mathrm{CO}_{2}$ synthesis process is probably involved, but the $\mathrm{CO}_{2}$ formation mechanism has not been identified. Recent observations indicate that $\mathrm{CO}_{2}$ (ice) formation is linked to $\mathrm{H}_{2} \mathrm{O}$ formation[2, 3]. Theoretical and laboratory studies suggested an efficient $\mathrm{CO}+$

$\mathrm{OH} \rightarrow \mathrm{CO}_{2}+\mathrm{H}$ reaction in $\mathrm{CO} / \mathrm{H}_{2} \mathrm{O}$ mixed ices due to its low activation energy barrier[4-9], however the limited mobility of $\mathrm{CO}$ and $\mathrm{OH}$ species at low temperatures inhibits this reaction probability. Carbon monoxide is the second most abundant gas species after hydrogen in the (ISM)[10]. It may well be the parent molecule for the synthesis of $\mathrm{CO}_{2}$, as well as for many organic molecules in space[11]. Here we report an efficient surface reaction pathway for gas phase $\mathrm{CO}$ conversion to solid $\mathrm{CO}_{2}$ on amorphous solid water (ASW) films or crystalline water ice films at $76 \mathrm{~K}$, activated by ice absorption of Lyman-a (10.2 eV) radiation. A direct Eley-Rideal process[12-14] which does not involve prior energetic accommodation of $\mathrm{CO}$ by the ice surface is observed, when the incident $\mathrm{CO}$ molecules interact with surface-bound $\mathrm{OH}$ radicals that are produced by water photodissociation. The surface reaction is kinetically separate from the previously-studied solid phase $\mathrm{CO}+\mathrm{OH}$ reaction. This finding of a significant new process for $\mathrm{CO}_{2}$ production will influence kinetic models involving $\mathrm{CO}(\mathrm{g})$ consumption and $\mathrm{CO}_{2}$ (ice) formation in molecular clouds[4, 15] or in the atmospheres of icy moons in the solar system[16]. More generally, similar Eley- 
Rideal surface reactions on ice films which contain surface-bound radical species are likely to occur for other gas molecules in space.

Photochemical processes are known to drive molecular synthesis in the ISM, especially inside thin ice films condensed on dust grains[17-19]. During photodissociation of mixed $\mathrm{CO} / \mathrm{H}_{2} \mathrm{O}$ ices, hydroxyl radicals are produced that can interact with $\mathrm{CO}$. This reaction has been studied in the laboratory by hydrogenation of $\mathrm{CO} / \mathrm{O}_{2}$ binary ices[6,7] and by $\mathrm{CO}$ reaction with non-energetic $\mathrm{OH}$ radicals[8,9]. In contrast to these studies of bulk ices containing condensed $\mathrm{CO}$ molecules, here we report that gas phase $\mathrm{CO}$ has a remarkable reactivity with photochemically-generated $\mathrm{OH}$ radicals on the surface of $\mathrm{H}_{2} \mathrm{O}$ ice. This finding reveals a significant new kinetic process for $\mathrm{CO}_{2}$ (ice) formation in space, especially in the star forming regions of the ISM before $\mathrm{CO}$ freeze-out[20].

The classical Eley-Rideal (E-R) mechanism for a surface reaction involves, in its simplest form, the direct collision of an incoming species from the gas phase with a surface-bound species to produce a product molecule and was originally proposed as the mechanism for a metal-catalyzed surface reaction[12]. This type of reaction occurs with reactive incoming species such as atomic $H$ [21]. In space, it is proposed that molecular $\mathrm{H}_{2}$ formation by $\mathrm{H}+\mathrm{H}$ recombination in high temperature ISM regions occurs efficiently through the E-R mechanism[22]. A modification of the classical direct Eley-Rideal reaction involves an incoming species which partially accommodates on the surface as a mobile precursor and, in its subsequent diffusive motion, encounters an immobile reactive partner which then participates with the mobile species in a chemical reaction [23]. We present kinetic evidence that the $\mathrm{CO}+\mathrm{OH}$ (on water ice) reaction occurs by a direct EleyRideal process not involving CO surface mobility. 


\subsection{Experimental}

Figure 2.1 shows an experiment which demonstrates the photochemicallyinduced reactivity toward $\mathrm{CO}(\mathrm{g})$ of a $20 \mathrm{~nm}$ thick amorphous solid water (ASW) film. The $\mathrm{H}_{2} \mathrm{O}$ film is deposited at $76 \mathrm{~K}$ on a $0.3 \mathrm{~cm}^{2}$ disk of pressed $\mathrm{KBr}$ powder held on a tungsten grid in a high vacuum stainless steel cell of $1.9 \times 10^{-3} \mathrm{~m}^{3}$ volume[24]. In addition, crystalline ice films were made by heating deposited ASW to $170 \mathrm{~K}$ until the phase transition is completed and then cooling to $76 \mathrm{~K}$ for similar studies. Lyman-a radiation $\left(2.7 \times 10^{18}\right.$ photons $\left.\mathrm{m}^{-2} \mathrm{~s}^{-1}\right)$ [25, 26], passing through a LiF window and an internal aperture, is incident at $45^{\circ}$ to the ice film. Transmission IR spectroscopy at $45^{\circ}$ incidence angle to the ice surface is performed during the irradiation to observe the formation of condensed $\mathrm{CO}_{2}$ product. A quadrupole mass spectrometer (QMS) senses the pressure of flowing $\mathrm{CO}(\mathrm{g})$ before, during and after irradiation. $\mathrm{A}$ mixture of $\mathrm{C}^{16} \mathrm{O}$ and $\mathrm{C}^{18} \mathrm{O}$ is employed with a mole fraction, $X_{C 180}$. We measured the $C^{18} O(m / z=30)$ behavior and converted it to total $\mathrm{CO}$ pressure as shown in Figure 2.1. The CO partial pressure is established over the ice film using a measured $\mathrm{CO}$ pumping speed of $2 \times 10^{-4} \mathrm{~m}^{3} \mathrm{~s}^{-1}$ [27]. When the UV light is admitted, CO begins to be consumed immediately and a steady state CO pressure is reached in about $300 \mathrm{~s}$. When the UV light is extinguished, the CO pressure rises back to the original value in about $300 \mathrm{~s}$. This behavior is observed at many CO pressures and the experiment closely resembles the well-known King-Wells method used in surface science to measure adsorption kinetics[28]. The change of $C O$ pressure $\left(\Delta \mathrm{P}_{C O}\right)$ is found to be similar $( \pm 20 \%)$ with both thick and thin ASW films, as well as on a crystalline ice film, indicating the reaction is occurring on the ice surface and that the ice structure is not an important factor. 


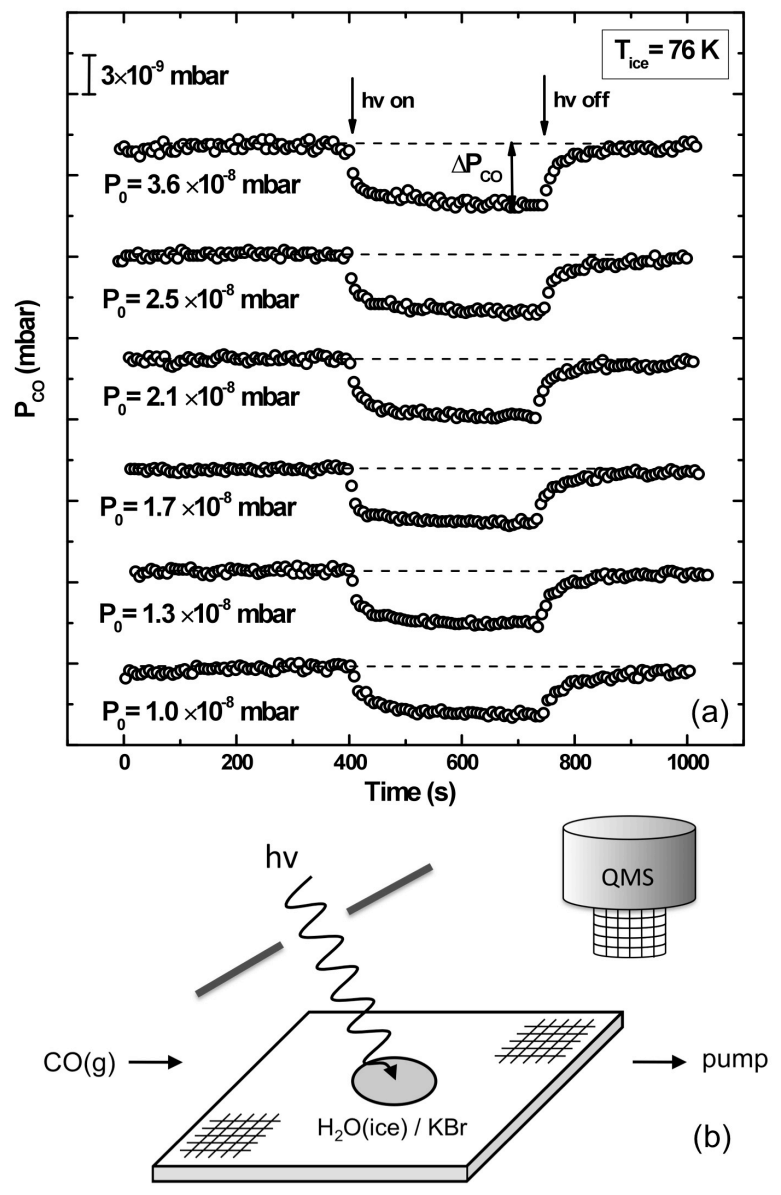

Figure 2.1. (a) $\mathrm{CO}(\mathrm{g})$ consumption during experiments at various $\mathrm{CO}$ pressures at $76 \mathrm{~K}$. (b) Schematic of $\mathrm{CO}(\mathrm{g})$ consumption measurement procedure on irradiated ASW.

Figure 2.2 shows the photochemical production of condensed $\mathrm{CO}_{2}$ isotopomers during the experiment where $\mathrm{X}_{\mathrm{C} 180}=\mathrm{X}_{\mathrm{C} 160}=0.50 \pm 0.05$. The strong $\mathrm{v}_{3}$ modes for $\mathrm{C}^{16} \mathrm{O}_{2}$ and $\mathrm{C}^{18} \mathrm{O}^{16} \mathrm{O}$ are observed. Their frequencies are close to the typical frequencies of pure $\mathrm{CO}_{2}$ isotopomer ices[29, 30], showing that the solid $\mathrm{CO}_{2}$ product is formed on the outer $\mathrm{H}_{2} \mathrm{O}$ ice surface. It is seen that the two $\mathrm{CO}_{2}$ absorption bands grow monotonically. Plots of their integrated absorbance over time display the same slope, indicating that any ${ }^{18} \mathrm{O}$ isotope effect for $\mathrm{CO}$ is negligible[24]. 


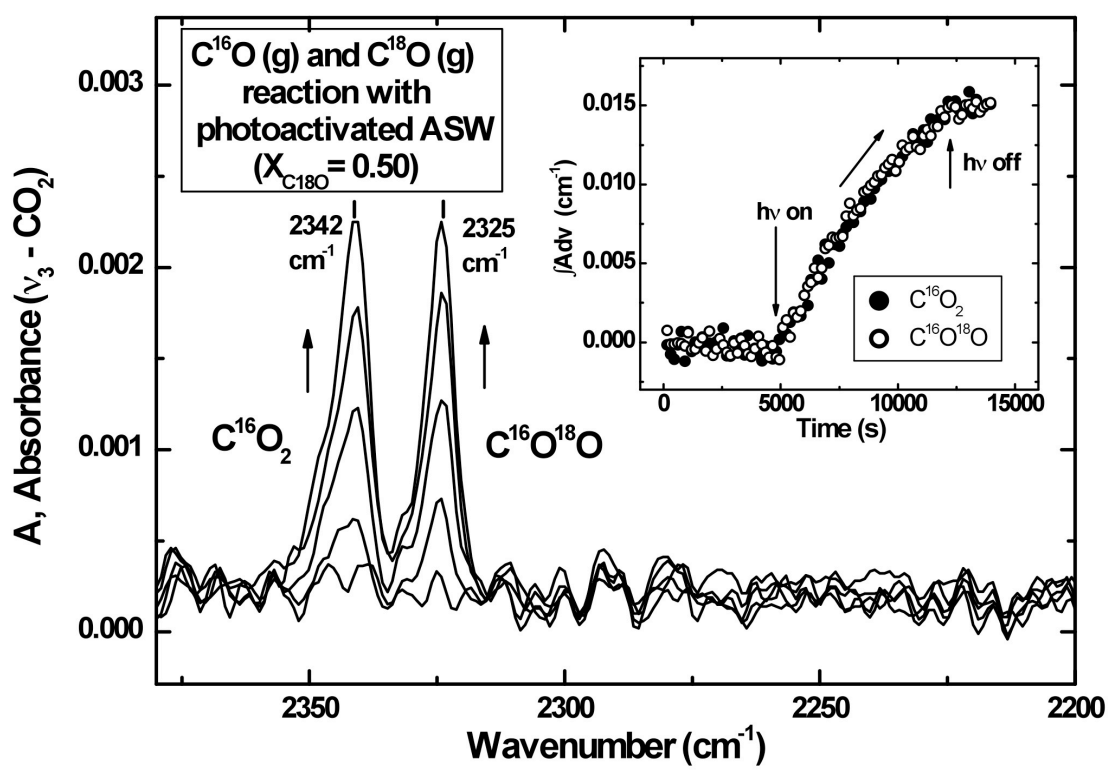

Figure 2.2. IR spectra of $\mathrm{C}^{16} \mathrm{O}_{2}$ and $\mathrm{C}^{16} \mathrm{O}^{18} \mathrm{O}$ isotopomer ices produced during $\mathrm{C}^{16} \mathrm{O}(\mathrm{g})$ and $\mathrm{C}^{18} \mathrm{O}(\mathrm{g})$ reaction on irradiated $\mathrm{ASW}$. The inset shows the growth of integrated absorbance of $\mathrm{C}^{16} \mathrm{O}_{2}$ and $\mathrm{C}^{16} \mathrm{O}^{18} \mathrm{O}$ isotopomers vs. time.

\subsection{Results and Discussion}

The rate of $\mathrm{CO}(\mathrm{g})$ consumption, $\mathrm{R}_{\mathrm{CO}}$ (molecules $\mathrm{m}^{-2} \mathrm{~s}^{-1}$ ), can be found by measuring the change of $C O$ pressure $\left(\Delta \mathrm{P}_{\mathrm{CO}}\right)$ during $\mathrm{UV}$ irradiation. The $\Delta \mathrm{P}_{\mathrm{CO}}$ is directly proportional to $R_{c o}$ in a rapidly pumped system[31], as shown in eqn (2.1):

$$
R_{C O}=\frac{\Delta P_{C O} \cdot S_{C O}}{A \cdot k_{B} T_{g}}
$$

where $S_{C O}$ is the measured pumping speed of $\mathrm{CO}(\mathrm{g}), A$ is the irradiated sample area, $\mathrm{k}_{\mathrm{B}}$ is the Boltzmann constant, and $\mathrm{T}_{\mathrm{g}}$ is the $\mathrm{CO}$ gas temperature. At any $\mathrm{CO}$ 
pressure, the $\mathrm{CO}$ consumption rate during irradiation is proportional to the accumulated surface $\mathrm{OH}(\mathrm{a})$ coverage, $\mathrm{N}_{\mathrm{OH}}$. When the reaction reaches steady state, the surface-bound $\mathrm{OH}(\mathrm{a})$ production rate is equal to its consumption rate, including the $\mathrm{OH}(\mathrm{a})$ reaction rate with $\mathrm{CO}(\mathrm{g})$, and the $\mathrm{OH}(\mathrm{a})$ recombination rate with $\mathrm{H}(\mathrm{a})$ or $\mathrm{OH}(\mathrm{a})$. Molecular dynamics studies show a large fraction of photoproduced $\mathrm{H}(\mathrm{a})$ desorbs from the ice surface because of its weak binding energy[32], leaving an $\mathrm{OH}(\mathrm{a})$-enriched surface. We therefore postulate that the $\mathrm{OH}(\mathrm{a})+\mathrm{OH}(\mathrm{a}) \rightarrow \mathrm{H}_{2} \mathrm{O}_{2} \rightarrow \mathrm{H}_{2} \mathrm{O}+\mathrm{O}$ reaction is the major process that consumes the excess $\mathrm{OH}(\mathrm{a})[33$ ]. After irradiation, the $\mathrm{OH}(\mathrm{a})$ species remaining on the surface continue to be consumed by $\mathrm{CO}(\mathrm{g})$ and by the recombination reactions, resulting in a decrease of $R_{C O}$. An empirical fit (see Supplementary Information) with second-order kinetics gives $N_{O H}^{S S}=(4 \pm 1) \times 10^{17} \mathrm{~m}^{-2}$ from the data in six measurements at steady state, corresponding to a fractional surface $\mathrm{OH}$ coverage of $\theta_{O H}^{S S}=0.05 \mathrm{ML}$ (monolayer) at steady state.

The incident flux $\mathrm{F}_{\mathrm{co}}$ of $\mathrm{CO}(\mathrm{g})$ on the ice surface is calculated using eqn (2.2):

$$
F_{C O}=\frac{P_{C O}^{0}}{\left(2 \pi m k_{B} T\right)^{1 / 2}}
$$

where $P_{C O}^{0}$ is the total pressure of $\mathrm{CO}(\mathrm{g})$ before irradiation, and $\mathrm{m}$ is the mass of the $\mathrm{CO}$ molecule. Therefore, the cross section, $\sigma_{\mathrm{CO}}$, for $\mathrm{CO}(\mathrm{g})$ reaction can be obtained as $d[C O] / d t=F_{C O} \cdot \sigma_{C O} \cdot N_{O H}=R_{C O}$. The reaction cross section, $\sigma_{C O}$, is found to be $6 \times 10^{-20} \mathrm{~m}^{2}$, which is of the order of a geometrical molecular cross section, indicating a direct Eley-Rideal type process.

When $\mathrm{CO}$ molecules strike the ice surface, their average residence time can be calculated from equation (2.3):

$$
t_{s}=\frac{1}{v} e^{E_{d e s} / k_{B} T}
$$


where $v$ is the desorption frequency factor $\approx 10^{12} \mathrm{~s}^{-1}, \mathrm{E}_{\text {des }}$ is the CO desorption energy from the $\mathrm{H}_{2} \mathrm{O}$ ice surface, and $\mathrm{T}$ is the ice temperature. The desorption energy is $E_{\text {des }}=0.125 \mathrm{eV}$ at low $\mathrm{CO}$ coverage[34]. Therefore at $\mathrm{T}=76 \mathrm{~K}$, the residence time for $\mathrm{CO}(\mathrm{g})$ on the $\mathrm{H}_{2} \mathrm{O}$ ice surface is $\sim 2 \times 10^{-4} \mathrm{~s}$. The fractional coverage of $\mathrm{CO}$ adsorbed on the $\mathrm{H}_{2} \mathrm{O}$ ice surface at a given $\mathrm{CO}(\mathrm{g})$ pressure is $\theta_{\mathrm{CO}}=\left(F_{\mathrm{CO}} \times t_{\mathrm{s}}\right) / \mathrm{N}_{\mathrm{H} 2 \mathrm{O}}$, where $\mathrm{N}_{\mathrm{H} 2 \mathrm{O}} \approx 9 \times 10^{18} \mathrm{~m}^{-2}$ is the surface density of $\mathrm{H}_{2} \mathrm{O}$ adsorption sites based on the density of ASW. Under our experimental conditions, the $\mathrm{CO}$ fractional coverage is only $\theta_{\mathrm{CO}}=1 \times 10^{-6} \mathrm{ML}$, i.e. 5 orders of magnitude smaller than the steady-state fractional $\mathrm{OH}$ coverage. This low coverage is consistent with the absence of the adsorbed CO IR absorption band. Thus it is not likely for the reaction to occur by a mechanism where $\mathrm{CO}$ adsorbs on the surface and translates to an $\mathrm{OH}(\mathrm{a})$ species.

The diffusion barrier $\left(\mathrm{E}_{\text {diff }}\right)$ for $\mathrm{CO}$ on the water ice surface is affected by ice surface structure and CO coverage. On ASW, $E_{\text {diff }}=0.10 \mathrm{eV}$ for the lowest CO coverage; the value drops to $0.050 \mathrm{eV}$ for crystalline ice[34], yielding a $\sim 50$ fold increase in the $\mathrm{CO}$ surface diffusion distance during its lifetime at $76 \mathrm{~K}$ for crystalline ice compared to ASW. In this work the observed CO reaction rates are similar on both ASW and crystalline ice, confirming that the involvement of a mobile $\mathrm{CO}$ precursor is unlikely. We conclude that the reaction mechanism is of the direct Eley-Rideal type, where a $\mathrm{CO}(\mathrm{g})$ molecule reacts efficiently upon collision with a surface-OH species.

Figure 2.3 shows the temperature dependence of the reaction rate. As temperature decreases the $\mathrm{CO}$ consumption rate increases. A negative apparent activation energy of $-0.058 \mathrm{eV}$ is found. This is additional evidence to support the Eley-Rideal reaction mechanism: the negative activation energy indicates that activated diffusion processes for the reactants are not kinetically significant at 76 $\mathrm{K}$ for the $\mathrm{CO}+\mathrm{OH}$ reaction. We postulate that this temperature effect is due to the increased loss of steady-state surface $\mathrm{OH}(\mathrm{a})$ concentration with increasing 
temperature, as the activated recombination reactions occur, competing with the $\mathrm{CO}(\mathrm{g})+\mathrm{OH}(\mathrm{a})$ reaction and lowering its rate. The formation of the stable intermediate compound $\mathrm{HOCO}$ at unobservable concentrations is probably involved[6, 35].

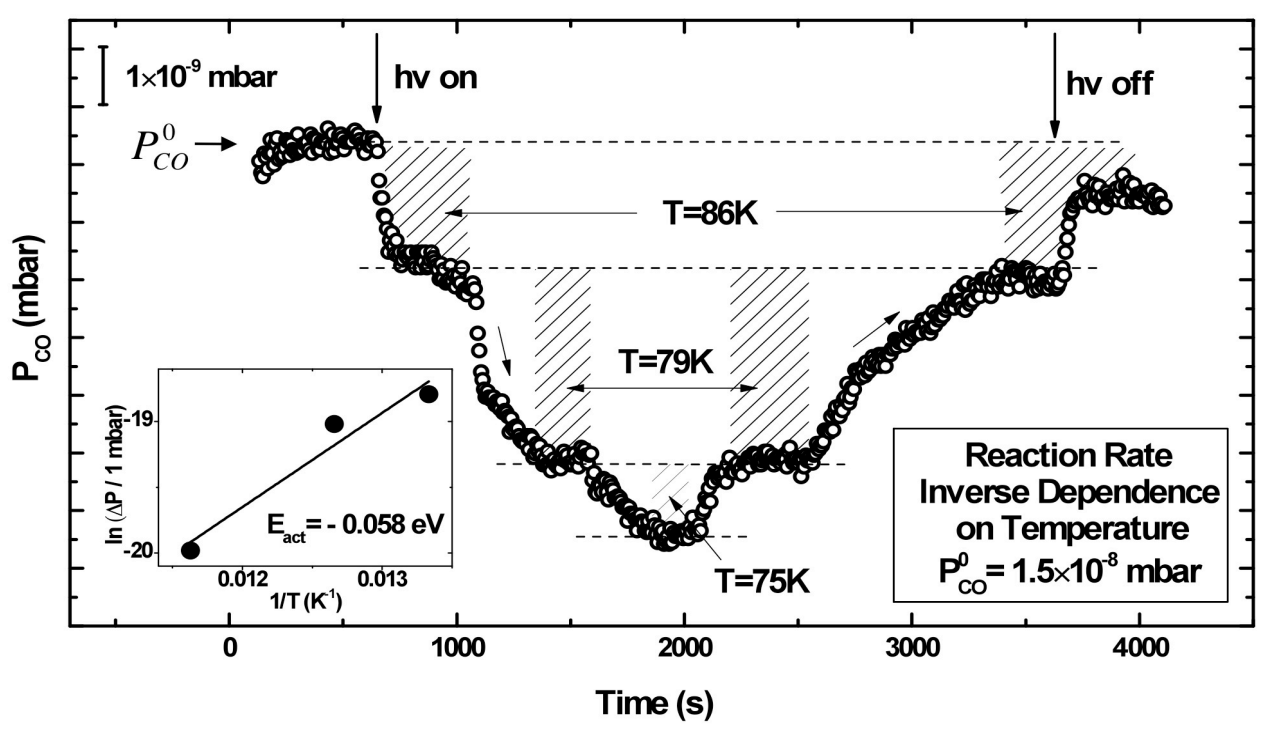

Figure 2.3. Temperature effect on $\mathrm{CO}(\mathrm{g})$ consumption rate between $75 \mathrm{~K}$ and $86 \mathrm{~K}$. The inset shows the Arrhenius plot giving a negative apparent activation energy, possibly caused by $\mathrm{OH}(\mathrm{a})$ consumption by recombination process.

\subsection{Modeling}

A kinetic model, described in the Supplemental Information, was used to fit the data of Figure 2.1 as shown in Figure 2.4. The model considers the flux of incident light, the cross section for $\mathrm{H}_{2} \mathrm{O}$ photodissociation, and the incoming flux of $\mathrm{CO}(\mathrm{g})$ in the system of known pumping speed to calculate the solid curve which is superimposed on the data for one measurement. It is found that the rate of the $\mathrm{OH}(\mathrm{a})+\mathrm{OH}(\mathrm{a})$ reaction on the irradiated ice is mainly responsible for the 
shape of the $\mathrm{P}_{\mathrm{CO}}$ curve, in agreement with the observation in Figure 2.1 that these curves are only weakly dependent on $P_{C O}^{0}$. A schematic for the reaction of photo-produced $\mathrm{OH}(\mathrm{a})$ with $\mathrm{CO}(\mathrm{g})$ via a direct Eley-Rideal process is shown in the inset of Figure 2.4.

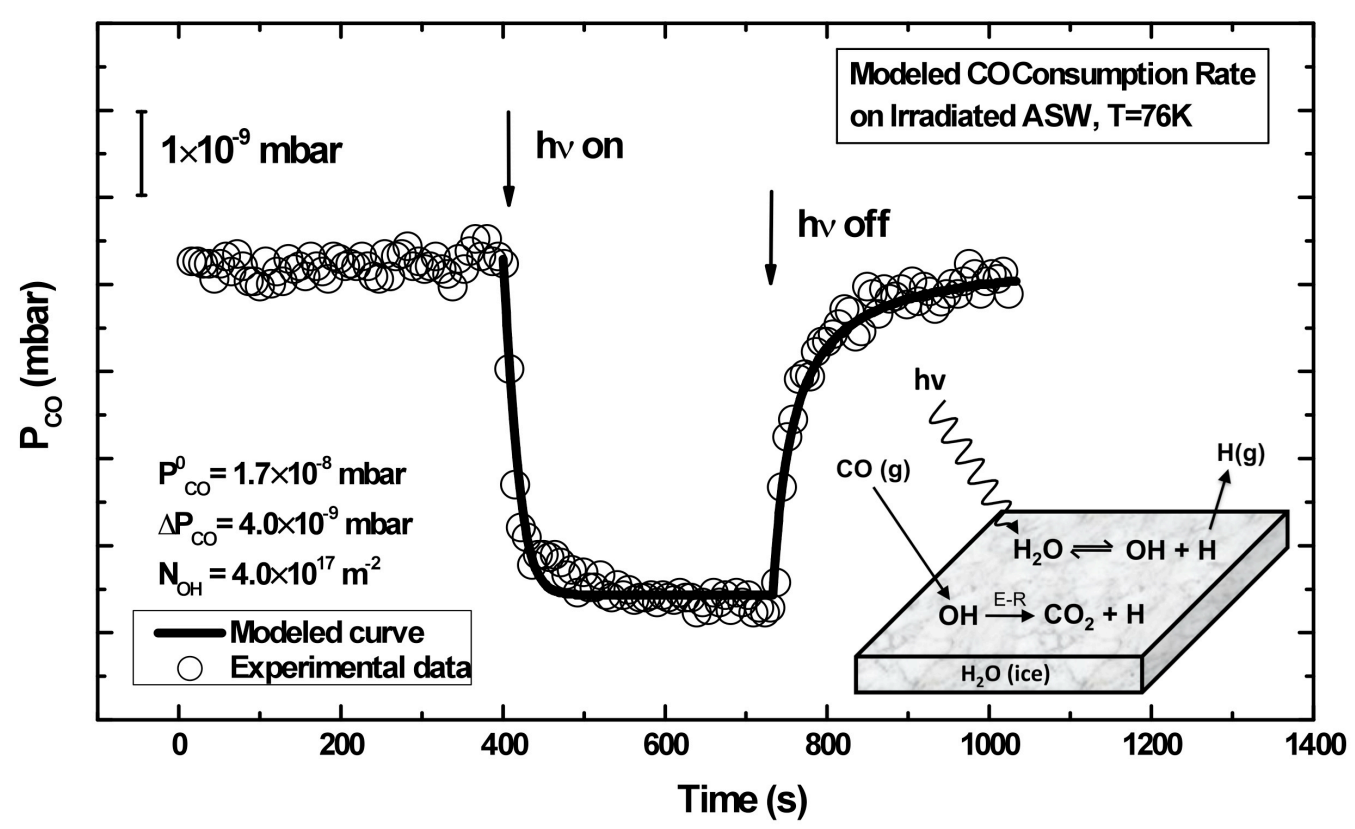

Figure 2.4. Modeled $\mathrm{CO}(\mathrm{g})$ consumption rate on irradiated $\mathrm{ASW}$ and its comparison to experimental data. The inset shows the schematic of the surface processes on the ice surface.

To study the kinetics of the $\mathrm{CO}$ consumption process, $\mathrm{CO}(\mathrm{g})+\mathrm{OH}(\mathrm{a}) \rightarrow$ $\mathrm{CO}_{2}$ (ice) $+\mathrm{H}$, we divide the experimental $\mathrm{CO}$ pressure curves (as shown in Figure 2.1) into two processes. Process I describes the CO pressure decay to steady state during irradiation and Process II describes the CO pressure recovery after irradiation ends.

As mentioned in the paper, the $\mathrm{CO}(\mathrm{g})$ consumption rate, $\mathrm{R}_{\mathrm{co}}$, is proportional to the initial $\mathrm{CO}$ pressure and the surface $\mathrm{OH}$ coverage, $\mathrm{N}_{\mathrm{OH}}$. 


$$
R_{C O}=\frac{\Delta P_{C O} \cdot S_{C O}}{A \cdot k_{B} T_{g}}=k_{1} P_{C O} N_{O H}
$$

Because of the observed weak dependence of $\mathrm{R}_{\mathrm{CO}}$ on $\mathrm{CO}$ pressure, $\mathrm{P}_{\mathrm{CO}}$, and the apparent negative activation barrier, we propose that the $\mathrm{CO}(\mathrm{g})+\mathrm{OH}(\mathrm{a})$ reaction only consumes a small portion of $\mathrm{OH}$ species on the surface. The majority of surface $\mathrm{OH}$ species are consumed by the competitive $\mathrm{OH}(\mathrm{a})+\mathrm{OH}(\mathrm{a})$ second-order recombination process. This assumption is confirmed by the following analysis.

In Process II, we assume the $\mathrm{OH}$ consumption rate is simply determined by the $\mathrm{CO}(\mathrm{g})+\mathrm{OH}(\mathrm{a})$ reaction rate and by the $\mathrm{OH}(\mathrm{a})+\mathrm{OH}(\mathrm{a})$ recombination rate, despite other complex processes that could occur in an irradiated water ice.

$$
-\frac{d N_{O H}}{d t}=R_{C O}+R_{O H+O H}=k_{1} P_{C O} N_{O H}+k_{2}\left(N_{O H}\right)^{2}
$$

Because the $\mathrm{CO}(\mathrm{g})+\mathrm{OH}(\mathrm{a})$ reaction rate is relatively slow, the $\mathrm{OH}$ consumption rate is approximately a second-order kinetic process. The empirical secondorder kinetic fits of $1 / R_{C O}$ versus time from the experimental data are consistent for a range of pressures, as seen in Figure 2.5. We use this simplified secondorder kinetics assumption in our further modeling. 


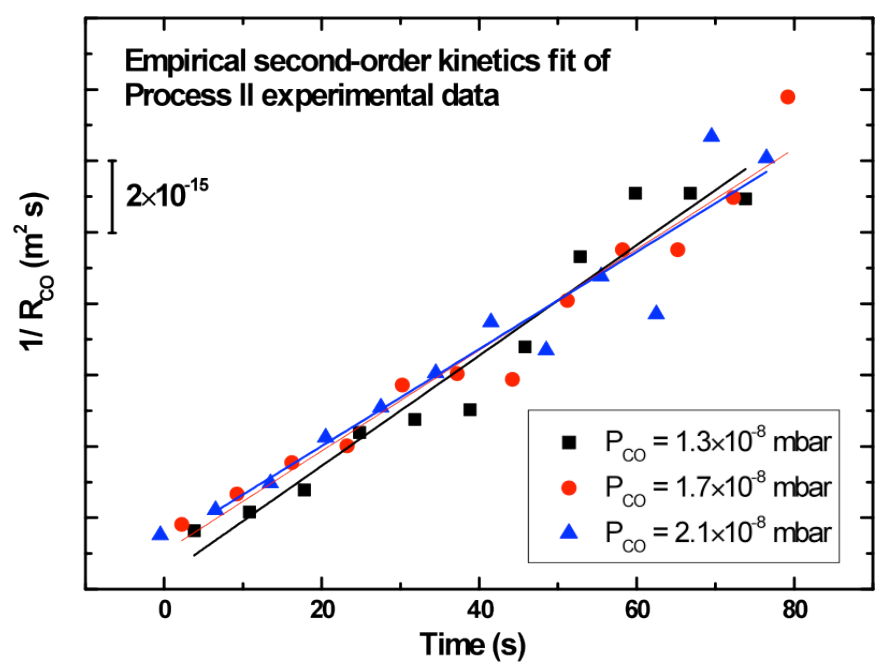

Figure 2.5. Empirical second-order kinetic fits of Process II experimental data.

By integrating equation (2.5):

$$
-\int_{N_{O H}^{S S}}^{N_{O H}} \frac{d N_{O H}}{k_{1} P_{C O} N_{O H}+k_{2}\left(N_{O H}\right)^{2}}=\int_{0}^{t} d t
$$

where $N_{O H}^{S S}$ is the $\mathrm{OH}$ coverage during irradiation at steady state, we get

$$
N_{O H}=\frac{k_{1} P_{C O} N_{O H}^{S S}}{k_{1} P_{C O} e^{k_{1} P_{C O} t}+k_{2} N_{O H}^{S S}\left(e^{k_{1} P_{C O} t}-1\right)}
$$

In Process I, the $\mathrm{OH}$ consumption rate is equilibrated with its production rate by irradiation. The kinetic equation is:

$$
\frac{d N_{O H}}{d t}=F_{h v} \sigma_{\mathrm{H}_{2} \mathrm{O}} N_{\mathrm{H}_{2} \mathrm{O}}-k_{1} P_{\mathrm{CO}} N_{\mathrm{OH}}-k_{2}\left(N_{\mathrm{OH}}\right)^{2}
$$

where $F_{h v}$ is the flux of photons, $\sigma_{\mathrm{H} 2 \mathrm{O}}=1 \times 10^{-21} \mathrm{~m}^{2}$ is the photodissociation cross section of $\mathrm{H}_{2} \mathrm{O}$ ice, and $\mathrm{N}_{\mathrm{H} 2 \mathrm{O}}$ is the surface density of $\mathrm{H}_{2} \mathrm{O}$ molecules. Here we 
assume that only the first layer of water ice contributes to the surface $\mathrm{OH}$ production. Integrating eqn (2.8):

$$
\int_{0}^{N_{O H}} \frac{d N_{O H}}{F_{h v} \sigma_{H_{2} O} N_{H_{2} O}-k_{1} P_{C O} N_{O H}-k_{2}\left(N_{O H}\right)^{2}}=\int_{0}^{t} d t
$$

we get

$$
N_{O H}=\frac{2 c\left(1-e^{\sqrt{k_{1}^{2} P_{C O}^{2}+4 k_{2} c} \cdot t}\right)}{k_{1} P_{C O}\left(1-e^{\sqrt{k_{1}^{2} P_{C O}^{2}+4 k_{2} c} \cdot t}\right)-\sqrt{k_{1}^{2} P_{C O}^{2}+4 k_{2} c}\left(1+e^{\sqrt{k_{1}^{2} P_{C O}^{2}+4 k_{2} c} \cdot t}\right)}
$$

where $c=F_{h v} \sigma_{H_{2} O} N_{H_{2} \mathrm{O}} \approx 2 \times 10^{16} \mathrm{~m}^{-2} \mathrm{~s}^{-1}$.

At steady state,

$$
F_{h v} \sigma_{H_{2} O} N_{H_{2} O}=k_{1} P_{C O} N_{O H}^{S S}+k_{2}\left(N_{O H}^{S S}\right)^{2}
$$

Here we assume a steady state surface $\mathrm{OH}$ concentration, $N_{O H}^{S S}$, so that the rate constants $k_{1}$ and $k_{2}$ can be calculated from eqn (2.4) and (2.11). These rate constants are used in eqn (2.7) and (2.10) to model the curves to best fit the experimental data.

The value of $N_{O H}^{S S}$ can be estimated from the empirical second-order kinetics fit in Figure 2.5, and then be slightly adjusted to best fit the experimental data. An optimum $N_{O H}^{S S}=(4 \pm 1) \times 10^{17} \mathrm{~m}^{-2}$ is obtained from the data of six measurements. The fitting result is shown in Figure 2.4 for one experiment. The modeled curve for Process II fits the experimental data well, while the curve for Process I has a small deviation from the data when $\mathrm{P}_{\mathrm{CO}}$ approaches the steady state. This may be rationalized by postulating a small contribution from slow diffusion of $\mathrm{OH}$ from the underlying layers of the water ice. Since the Lyman- $\alpha$ light penetrates into the ice, the diffusing $\mathrm{OH}$ species produced in the bulk may contribute to the surface 
$\mathrm{OH}$ concentration, causing a slightly increasing $\mathrm{CO}$ consumption rate in the plateau region of Figures 2.1 and 2.4.

With the calculated values of $\mathrm{k}_{1}$ and $\mathrm{k}_{2}$, the $\mathrm{OH}$ consumption rate by the $\mathrm{CO}(\mathrm{g})$ $+\mathrm{OH}(\mathrm{a})$ reaction and by the $\mathrm{OH}(\mathrm{a})+\mathrm{OH}(\mathrm{a})$ recombination are $3 \%$ and $97 \%$ of the total $\mathrm{OH}$ production rate by irradiation, respectively. Therefore our previous assumptions that the $\mathrm{CO}(\mathrm{g})+\mathrm{OH}(\mathrm{a})$ reaction rate is relatively small and that the $\mathrm{OH}$ consumption rate can be simplified as a second-order kinetic process are reasonable.

The use of a mass spectrometer to observe the rate of processes occurring at an ice surface provides a highly sensitive kinetic method. Measurements of the CO reaction rates, such as those shown here, have a sensitivity of the order of $10^{-7} \mathrm{ML} \mathrm{s}^{-1}$ or $\sim 10^{12}$ molecules $\mathrm{m}^{-2} \mathrm{~s}^{-1}$, whereas measurements of the photochemical rate of production of a molecule in the ice bulk, using the widelyapplied IR measurement method, have a sensitivity of only $\sim 10^{17}$ molecules $\mathrm{m}^{-2}$ $\mathrm{s}^{-1}$ if a strong vibrational mode is measured[24].

\subsection{Summary}

In summary we have observed the reaction between photochemicallyproduced surface-bound $\mathrm{OH}$ species and incident $\mathrm{CO}(\mathrm{g})$ molecules at water ice surfaces at $76 \mathrm{~K}$. The reaction is found to occur by a direct Eley-Rideal mechanism where a $\mathrm{CO}(\mathrm{g})$ molecule collides and reacts directly with a surface $\mathrm{OH}$ species to produce $\mathrm{CO}_{2}$ (ice). This observation may explain the origin of much of the $\mathrm{CO}_{2}$ (ice) observed in the dense ISM as well as the $\mathrm{CO}$ consumption in the atmospheres of icy moons. In our solar system, $\mathrm{CO}_{2}$ and $\mathrm{O}_{2}$ are observed in the exosphere of Saturn's satellite Rhea by the Cassini spaceflight[16]. Our experiments demonstrate an efficient process to convert $\mathrm{CO}(\mathrm{g})$ to $\mathrm{CO}_{2}$ by reaction with surface $\mathrm{OH}$ species on astronomical ices. 


\subsection{References:}

1. van Dishoeck, E.F., et al., A search for interstellar gas-phase $\mathrm{CO}_{2}$. Gas: solid state abundance ratios. Astronomy and Astrophysics, 1996. 315: p. L349.

2. Öberg, K.I., et al., The Spitzer Ice Legacy: Ice Evolution from Cores to Protostars. Astrophys. J., 2011. 740: p. 109.

3. Pontoppidan, K.M., et al., The c2d Spitzer Spectroscopic Survey of Ices Around Low-Mass Young Stellar Objects. II. CO . Astrophys. J., 2008. 678: p. 1005.

4. Ruffle, D. and E. Herbst, New models of interstellar gas-grain chemistry, III. Solid $\mathrm{CO}_{2}$. Monthly Notices of the Royal Astronomical Society, 2001. 324: p. 1054.

5. Garrod, R.T. and T. Pauly, On The Formation of $\mathrm{CO}_{2}$ and Other Interstellar Ices. Astrophys. J., 2011. 735: p. 15.

6. Ioppolo, S., et al., Surface formation of $\mathrm{CO}_{2}$ ice at low temperatures. Monthly Notices of the Royal Astronomical Society, 2011. 413: p. 2281.

7. Noble, J.A., et al., $\mathrm{CO}_{2}$ Formation in Quiescent Clouds: An Experimental Study of The $\mathrm{CO}+\mathrm{OH}$ Pathway. Astrophys. J., 2011. 735: p. 121.

8. Oba, Y., et al., Experimental Study of $\mathrm{CO}_{2}$ Formation by Surface Reactions of Non-Energetic OH Radicals With CO Molecules. Astrophys. J., 2010. 712: p. L174.

9. Zins, E.-L., P.R. Joshi, and L. Krim, Reactivity Between a CO-Containing Ice and Non-Energetic OH Radicals. Astrophys. J., 2011. 738: p. 175.

10. Tielens, A.G.G.M., The Physics and Chemistry of the Interstellar Medium, 2005, Cambridge, UK: Cambridge University Press.

11. Ehrenfreund, P. and S.B. Charnley, Organic Molecules in The Interstellar Medium, Comets, and Meteorites: A Voyage from Dark Clouds to the Early Earth. Annu. Rev. Astron. Astrophys., 2000. 38: p. 427.

12. Rideal, E.K., Proc. Cambridge Phil. Soc, 1938. 35: p. 130.

13. Eley, D.D., Adv. Catal. , 1948. 1: p. 157.

14. Eley, D.D. and E.K. Rideal, Nature, 1940. 146: p. 401. 
15. Whittet, D.C.B., P.F. Goldsmith, and J.L. Pineda, The Uptake of Interstellar Gaseous CO into Icy Grain Mantles in a Quiescent Dark Cloud. Astrophys. J., 2010. 720: p. 259.

16. Teolis, B., et al., Cassini finds an oxygen-carbon dioxide atmosphere at Saturn's icy moon Rhea. Science, 2010. 330(6012): p. 1813.

17. Gerakines, P.A., W.A. Schutte, and P. Ehrenfreund, Ultraviolet processing of interstellar ice analogs. I. Pure ices. Astron. Astrophys., 1996. 312: p. 289.

18. Ruffle, D.P. and E. Herbst, New models of interstellar gas-grain chemistry - II. Surface photochemistry in quiescent cores. Monthly Notices of the Royal Astronomical Society, 2001. 322: p. 770.

19. van Dishoeck, E.F., B. Jonkheida, and M.C. van Hemert, Photoprocesses in protoplanetary disks. Faraday Discussions, 2006. 133: p. 231.

20. Qi, C., et al., Imaging of the CO snow line in a solar nebula analog. Science, 2013. 341(6146): p. 630-632.

21. Cheng, C.C., et al., Atomic hydrogen-driven halogen extraction from silicon (100): Eley-Rideal surface kinetics. J. Am. Chem. Soc., 1992. 114: p. 1249.

22. Lemaire, J.L., et al., Competing Mechanisms of Molecular Hydrogen Formation In Conditions Relevant to The Interstellar Medium. Astrophys. J., 2010. 725: p. L156.

23. Harris, J. and B. Kasemo, On precursor mechanisms for surface reactions. Surface Science, 1981. 105: p. L281.

24. Yuan, C. and J.T. Yates, Jr., Isotope Effect in the Photochemical Decomposition of $\mathrm{CO}_{2}$ (ice) by Lyman-a Radiation. J. Chem. Phys., 2013. 138: p. 154302.

25. Rajappan, M., et al., Photochemical Decomposition of $\mathrm{N}_{2} \mathrm{O}$ by Lyman-a Radiation: Scientific Basis for a Chemical Actinometer. J. Phys. Chem. A, 2010. 114: p. 3443.

26. Rajappan, M., C. Yuan, and J.T. Yates, Jr., Lyman-a driven molecule formation on $\mathrm{SiO}_{2}$ surfaces - connection to astrochemistry on dust grains in the interstellar medium. J. Chem. Phys., 2011. 134: p. 064315.

27. Yuan, C. and J.T. Yates, Jr., Radiation Damage and Associated Phase Change Effect on Photodesorption Rates from Ices-Lya Studies of the Surface Behavior of $\mathrm{CO}_{2}$ (Ice). Astrophys. J., 2014. 780: p. 8. 
28. King, D.A. and M.G. Wells, Reaction Mechanism in Chemisorption Kinetics: Nitrogen on the $\{100\}$ Plane of Tungsten. Proc. R. Soc. Lond. A, 1974. 339: p. 245.

29. Falk, M., Amorphous solid carbon dioxide. J. Chem. Phys., 1987. 86: p. 560.

30. Escribano, R.M., et al., Crystallization of $\mathrm{CO}_{2}$ ice and the absence of amorphous $\mathrm{CO}_{2}$ ice in space. Proceedings of the National Academy of Sciences of the United States of America, 2013. 110(32): p. 12899.

31. Yates, J.T., Jr., The Thermal Desorption of Adsorbed Species, in Methods of Experimental Physics: Solid State Physics: Surfaces, R.L. Park and M.G. Lagally, Editors. 1985, Academic Press, Inc. p. 425.

32. Andersson, S. and E.F. van Dishoeck, Photodesorption of water ice: a molecular dynamics study. Astron. Astrophys., 2008. 491: p. 907.

33. Oba, Y., et al., Experimental studies of surface reactions among $\mathrm{OH}$ radicals that yield $\mathrm{H}_{2} \mathrm{O}$ and $\mathrm{CO}_{2}$ at 40-60 K. Phys. chem. chem. phys., 2011. 13: p. 15792.

34. Karssemeijer, L.J., et al., Dynamics of CO In Amorphous Water-Ice Environments. Astrophys. J., 2014. 781: p. 16.

35. Arasa, C., et al., Molecular dynamics simulations of $\mathrm{CO}_{2}$ formation in interstellar ices. J. Phys. Chem. A, 2013. 117(32): p. 7064. 


\section{Chapter 3: Isotope Effect in the Photochemical Decomposition of $\mathrm{CO}_{2}$ (ice) by Lyman-a Radiation}

Modified from manuscript published on J. Chem. Phys. 138, 154302 (2013)

\subsection{Introduction}

The photochemistry of molecules is often studied in frozen ice matrices where low temperatures suppress thermal reactions, allowing transient species to be preserved for study [1-3]. In the interstellar medium, mixed ices of molecules are present in the form of condensed layers held on the surface of the prevalent dust grains. Lyman-a radiation $(10.2 \mathrm{eV})$, originating in deep space from the recombination of $\mathrm{H}$ atoms and electrons, is a prominent form of radiation in space which drives photochemistry in ices, accompanied by the trapping of transient chemical species. As the ices are warmed at a later time in the history of the dust particles, photochemical products react and evaporate and the products may be detected in the gas phase by microwave and other spectroscopies. While thermal desorption from warming ices is responsible in some cases for the production of spectroscopically-observable gas phase molecules[4], recently the presence of these molecules in cold regions of interstellar space has been attributed to photodesorption processes from the ices by Oberg et al.[5]. The $\mathrm{CO}_{2}$ molecule, as well as its photodissociation products $\mathrm{CO}$ and $\mathrm{CO}_{3}$, have been widely observed in the space, and are believed to be important in interstellar chemistry [6-8].

The structure and isotopic composition of molecules detected in interstellar space is directly measured by microwave spectroscopy, and provides a method for categorizing the origin of the molecules found. Thus, as an example, the

${ }^{15} \mathrm{~N} /{ }^{14} \mathrm{~N}$ ratio is often measured for nitrogen-containing molecules such as $\mathrm{NH}_{3}$ and the ratio differs by a large amount for substances in the solar system 
compared to interstellar space [9]. The ${ }^{12} \mathrm{C} /{ }^{13} \mathrm{C}$ ratio was also investigated in comets, in the interstellar medium, and as well in different planetary nebulae. The cometary ratio, which is larger than 100 in some cases, is significantly higher than that observed in the interstellar clouds (ratio about 30-50). The study of the relative abundance of isotopes is important for us to understand the stellar nucleosynthesis and the chemical evolution of molecules in interstellar space and in the solar system [10-12].

We report that the Lyman-a $(10.2 \mathrm{eV})$ photodissociation of the $\mathrm{CO}_{2}$ molecule in $\mathrm{CO}_{2}$ (ice) occurs with a significantly larger $\mathrm{C}-\mathrm{O}$ bond dissociation rate for the ${ }^{12} \mathrm{C}$ isotopomer of $\mathrm{CO}_{2}$ compared to ${ }^{13} \mathrm{CO}_{2}$. This kinetic isotope effect (KIE) is postulated to occur as a result of isotopic differences in the probability of dissociating the $\mathrm{C}-\mathrm{O}$ bond in electronically-excited $\mathrm{CO}_{2}{ }^{*}$ molecules when in close contact with the ice matrix. The ice matrix supplies electronic quenching routes for the excited molecule. This leads to a mass-dependent rate of photodissociation favoring ${ }^{12} \mathrm{CO}_{2}$ over ${ }^{13} \mathrm{CO}_{2}$. Electronic quenching of the two isotopomers of the excited state of $\mathrm{CO}_{2}$ occurs with different probabilities by energy transfer to the $\mathrm{CO}_{2}$ matrix as the electronically-excited $\mathrm{CO}_{2}{ }^{*}$ molecule begins to dissociate along the upper-state repulsive potential curve associated with the excited state. Such effects are well known for molecules adsorbed on surfaces and may be understood in ices in the same mechanistic framework as developed for surfaces [13-17]. Similar quenching of electronically-excited molecules forms the basis for the establishment of the lifetime of a photoluminescent molecule in contact with its environment, and is a wellestablished relaxation phenomenon for electronically-excited molecules in many chemical environments [18]. 


\subsection{Experimental}

The apparatus used in this work has been described previously [19]. Briefly, a rf-excited quartz Lyman-a lamp using a $\mathrm{MgF}_{2}$ window and containing a flowing mixture of $10 \% \mathrm{H}_{2}$ and $90 \%$ Ar at $1.00 \pm 0.01$ Torr [20] is aimed into a stainless steel cell pumped by a turbopump (base pressure $=1 \times 10^{-8}$ Torr). The cell contains a reentrant Dewar. The Dewar supports a cryogenically-cooled W grid assembly (70\% transparent) containing $\mathrm{KBr}$ powder which has been compressed at 3000 psi into its openings as a $7 \mathrm{~mm}$ diameter disk. The $\mathrm{KBr}$ serves as an IRtransparent support for $\mathrm{CO}_{2}$ condensed from the gas to form a $\mathrm{CO}_{2}$ ice film. The grid, held between two Ni clamps, is shown in Figure 3.1a. The clamps supply efficient cooling from the reentrant Dewar and also provide electrical contact for ohmic heating of the sample by an electronic controller which senses the grid temperature from a type $\mathrm{K}$ thermocouple welded to the top center of the grid. The metal grid is in intimate contact with the small elements of compressed $\mathrm{KBr}$ as shown in Figure 3.1b. The grid supplies excellent heat transfer to the $\mathrm{KBr}$ compared to large $\mathrm{KBr}$ support plates often used for IR studies of condensed ices. Using $\mathrm{N}_{2}(\mathrm{I})$ which has been cooled by bubbling $\mathrm{He}[21], \mathrm{CO}_{2}$ ice films are condensed at $75 \mathrm{~K}$ on the $\mathrm{KBr}$ support through a doser that faces the front side of the $\mathrm{KBr}$ pressed disk. The ice has a thickness of $50 \mathrm{~nm}$ (nominal thickness measurement) as determined by the integrated IR absorbance of the $\mathrm{CO}_{2}\left(v_{1}+v_{3}\right.$ mode)[22]. The sample holder is mounted into the stainless steel IR cell containing differentially-pumped $\mathrm{KBr}$ windows for transmission IR measurements. The $7 \mathrm{~mm}$ diameter sample is accurately centered and oriented at $45^{\circ}$ to both the IR and the Lyman-a beams.

The cross section for Lyman- $\alpha$ radiation damage in the $\mathrm{CO}_{2}$ (ice) is an important factor in the estimation of photodepletion efficiency. Previous measurements present a large cross-section discrepancy and vary from $7.4 \times 10^{-}$ ${ }^{19} \mathrm{~cm}^{2}$ [23] to $4 \times 10^{-17} \mathrm{~cm}^{2}$ [24]. To verify the actual cross section in our situation, 
we measured the Lyman- $\alpha$ penetration depth by using a layered isotopomer ice. A $25 \mathrm{~nm}$ layer of ${ }^{12} \mathrm{CO}_{2}$ was deposited onto a $25 \mathrm{~nm}$ layer of ${ }^{13} \mathrm{CO}_{2}$ ice and from the decay of reaction rate of the bottom layer, we could estimate the Lyman- $\alpha$ penetration depth to be approximately $50 \mathrm{~nm}$, corresponding to a Lyman- $\alpha$ characteristic exponential absorption cross section of $9 \times 10^{-18} \mathrm{~cm}^{2}$. Therefore the $\mathrm{CO}_{2}$ ice at $50 \mathrm{~nm}$ thickness absorbs a significant fraction of the light intensity.
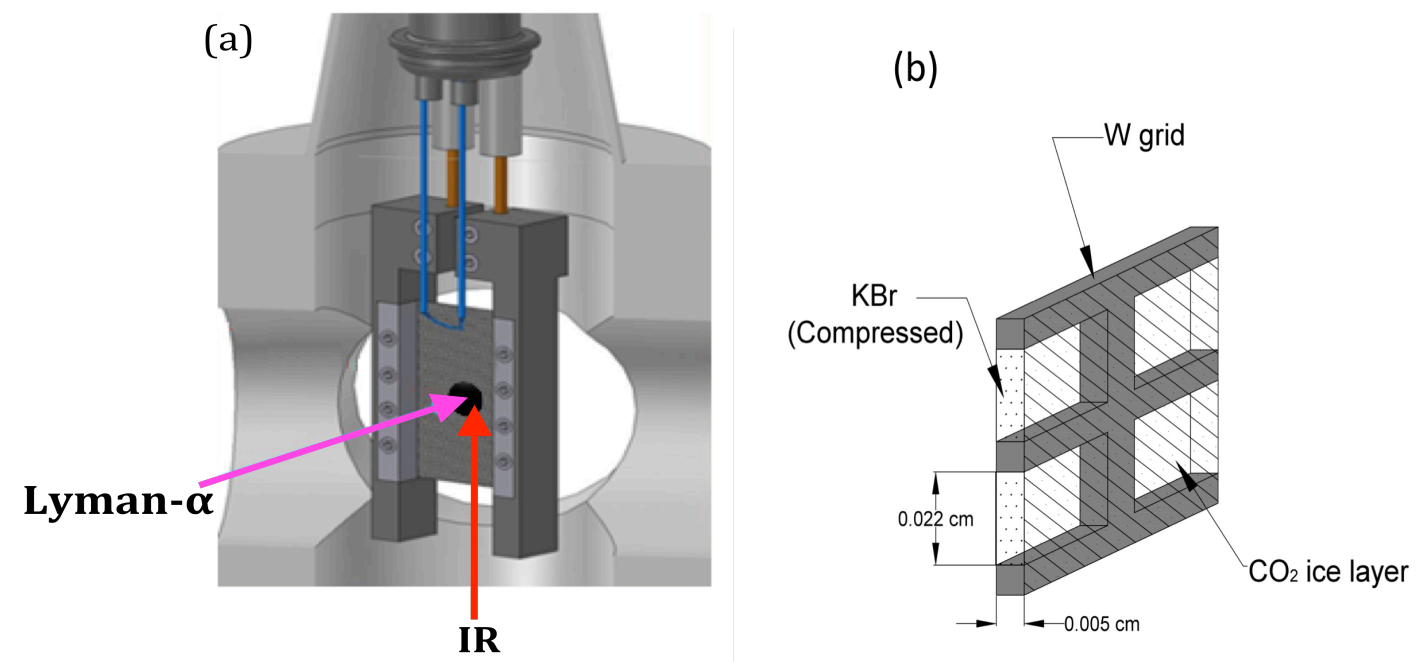

Figure 3.1. (a) Support of grid in Ni clamp with welded thermocouple, showing $90^{\circ}$ angle between Lyman- $\alpha$ and IR beam. (b) Side view of a section of the tungsten grid at high magnification showing pressed $\mathrm{KBr}$ support for ice condensation. Condensation occurs preferentially on one side of the $\mathrm{KBr}$ support as a result of dosing from a beam in the IR/UV cell.

The Lyman-a source delivers a flux of $2.7 \times 10^{14}$ photons $\cdot \mathrm{cm}^{-2} \cdot \mathrm{s}^{-1}$ of $10.2 \mathrm{eV}$ photons to the sample at the $45^{\circ}$ angle of incidence[19, 20]. The radiation is rather pure spectrally with a cutoff of the $\mathrm{MgF}_{2}$ window at $1100 \AA$, removing possible Ar emission lines. The lamp is very stable over months of operation as seen by the repeatability of the experimental measurements. 
The FT-IR spectrometer (Bruker Tensor 27) operates at $2 \mathrm{~cm}^{-1}$ resolution for product measurement and employs 200 scans to acquire a transmission IR spectrum in the range $500 \mathrm{~cm}^{-1}$ to $4000 \mathrm{~cm}^{-1}$. The spectrometer and the IR cell and external optics and detector are continuously purged with dry $\mathrm{CO}_{2}$-free air. An MCT-IR detector is employed operating at $77 \mathrm{~K}$.

$\mathrm{CO}_{2}$ is supplied by accurate volumetric dosing from a stainless steel gas handling system using Baratron capacitance manometers for pressure measurement. The gases are specified below: ${ }^{12} \mathrm{CO}_{2}--98.9 \%{ }^{12} \mathrm{C}, 1.1 \%{ }^{13} \mathrm{C}$; ${ }^{13} \mathrm{CO}_{2}--99 \%{ }^{13} \mathrm{C}, 1.0 \%{ }^{12} \mathrm{C},<3 \%{ }^{18} \mathrm{O}$, supplier: Sigma-Aldrich; ${ }^{12} \mathrm{C}^{18} \mathrm{O}_{2}-98.9 \%$ ${ }^{12} \mathrm{C}, 1.1 \%{ }^{13} \mathrm{C},<1 \%{ }^{16} \mathrm{O}$, supplier: Sigma-Aldrich. The accuracy of the isotopic compositions specified was verified by the quadrupole mass spectrometer in the vacuum system.

The infrared absorption bands of $\mathrm{CO}_{2}$ ices and their assignments are listed in Table 3.1. The intense $v_{3}$ mode shown in Figure 3.2 is broadened by lattice interactions with phonon modes in the ice[25]. Our studies of its behavior as a function of gas dose indicate that it exhibits non-linear behavior of integrated absorbance and absorbance related lineshape changes which make it inappropriate for kinetic measurements. In addition, during photolysis the lineshape is not constant. Therefore all $\mathrm{CO}_{2}$ kinetic measurements are made with the weaker $\left(v_{1}+v_{3}\right)$ mode shown in Figure 3.2 which exhibits an accurate linear integrated absorbance change with $\mathrm{CO}_{2}$ dose and constant lineshape as a function of integrated absorbance. 
Table 3.1. Infrared Absorptions of the Isotopic $\mathrm{CO}_{2}$ Ices and their Assignments.

\begin{tabular}{|c|c|c|c|}
\hline $\begin{array}{c}{ }^{12} \mathrm{CO}_{2} \text { Frequency } \\
\left(\mathrm{cm}^{-1}\right)\end{array}$ & $\begin{array}{c}{ }^{13} \mathrm{CO}_{2} \text { Frequency } \\
\left(\mathrm{cm}^{-1}\right)\end{array}$ & Assignment & Characterization \\
\hline 3710 & 3631 & $v_{1}+v_{3}$ & Combination mode \\
\hline 3603 & 3513 & $2 v_{2}+v_{3}$ & Combination mode \\
\hline 2345 & 2280 & $v_{3}$ & Asymmetric stretch \\
\hline 658 & 638 & $v_{2}$ & bending \\
\hline
\end{tabular}

A shutter within the cell is opened and closed to begin and end the photochemical experiments. IR spectra are recorded at 200 seconds intervals in the absorbance mode during irradiation and processed by subtracting the background spectra of the clean $\mathrm{KBr}$ and the $\mathrm{W}$ grid measured before $\mathrm{CO}_{2}$ is condensed. Integration of the IR spectra measured for photo-dissociation products was carried out for the kinetic measurements, where it was observed that the lineshape did not change significantly with absorbance either for $\mathrm{CO}_{2}$ dissociation products or for $\mathrm{CO}_{2}$. All spectral features measured during photolysis were normalized to the initial spectrum of the $\mathrm{CO}_{2}$ (ice) $\left(\int \mathrm{A}_{0} \mathrm{dv}\right)$ to yield measurements which were accurately comparable amongst the various experiments. By using a nominal 50\%-50\% mixture of the two isotopic species of $\mathrm{CO}_{2}$, accurate measurements of the relative rates of the kinetic processes could be made in order to determine the effect of ${ }^{12} \mathrm{C}$ - and ${ }^{13} \mathrm{C}$ - labeling in the $\mathrm{CO}_{2}$.

The possibility of the involvement of thermal radiant energy from the Lyman-a source was carefully checked by measuring the total radiant energy from the source with a bolometer placed in the cell at the sample position. When the Lyman-a source is operating a total emitted power of $75 \mu \mathrm{W} \cdot \mathrm{cm}^{-2}$ is measured. This is less than the known Lyman-a power emitted $\left(440 \mu \mathrm{W} \cdot \mathrm{cm}^{-2}\right)$ based on chemical actinometry as reported elsewhere[20]. An incandescent lamp, working 
at low output was substituted for the Lyman-a lamp and the rate of thermal desorption of a $50 \mathrm{~nm}$ thick layer of $\mathrm{CO}_{2}$ (ice) was carefully measured with white light operating at $375 \mu \mathrm{W} \cdot \mathrm{cm}^{-2}$ power output. The change in desorption rate at this power level was no more than $2.5 \%$ of the normal desorption rate obtained at $75 \mathrm{~K}$ in the dark, showing that the depletion of $\mathrm{CO}_{2}$ ice observed here by Lyman-a radiation is not significantly influenced by thermal effects from the Lyman-a lamp.

\subsection{Results}

Figure 3.2 shows the results for the photo-decomposition of a nominal 50\%$50 \%$ mixture of ${ }^{12} \mathrm{CO}_{2}$ and ${ }^{13} \mathrm{CO}_{2}$ condensed as a mixed ice on $\mathrm{KBr}$. The asymmetric stretching mode $\left(v_{3}\right)$ is shown for each isotopomer as well as the lower absorbance $\left(v_{1}+v_{3}\right)$ mode. Small $\mathrm{CO}_{2}$ isotopic impurity absorbances are indicated on the spectra. As photolysis occurs, the ${ }^{12} \mathrm{CO}_{2}$ and ${ }^{13} \mathrm{CO}_{2}$ absorbances disappear together and the infrared spectra of two isotopomer products, carbon monoxide ${ }^{12} \mathrm{CO}\left(2139 \mathrm{~cm}^{-1}\right)$ and ${ }^{13} \mathrm{CO}\left(2092 \mathrm{~cm}^{-1}\right)$, and carbon trioxide ${ }^{12} \mathrm{CO}_{3}(2045$ $\left.\mathrm{cm}^{-1}\right)$ and ${ }^{13} \mathrm{CO}_{3}\left(1990 \mathrm{~cm}^{-1}\right)$, are observed to form as reported by others in similar experiments[5, 26-28]. These products are produced by the breaking of a C-O bond in $\mathrm{CO}_{2}$, liberating $\mathrm{O}$ and $\mathrm{CO}$ which are trapped within the $\mathrm{CO}_{2}$ matrix and are designated $\mathrm{O}(\mathrm{Tr})$ and $\mathrm{CO}(\mathrm{Tr})$. Our observation of a significant ${ }^{12} \mathrm{C} /{ }^{13} \mathrm{C}$ isotope effect for both $\mathrm{CO}(\mathrm{Tr})$ and $\mathrm{CO}_{3}(\mathrm{Tr})$ formation lead to the mechanistic notion that the $\mathrm{O}(\mathrm{Tr})$ atom reacts with $\mathrm{CO}(\mathrm{Tr})$ to produce $\mathrm{CO}_{2}(\mathrm{Tr})$ which then reacts with a second $\mathrm{O}(\mathrm{Tr})$ atom to produce $\mathrm{CO}_{3}(\mathrm{Tr})$. Weak absorbance of $\mathrm{O}_{3}\left(1044 \mathrm{~cm}^{-1}\right)$ is also observed at $75 \mathrm{~K}$, as reported in lower temperature experiments [26]. 


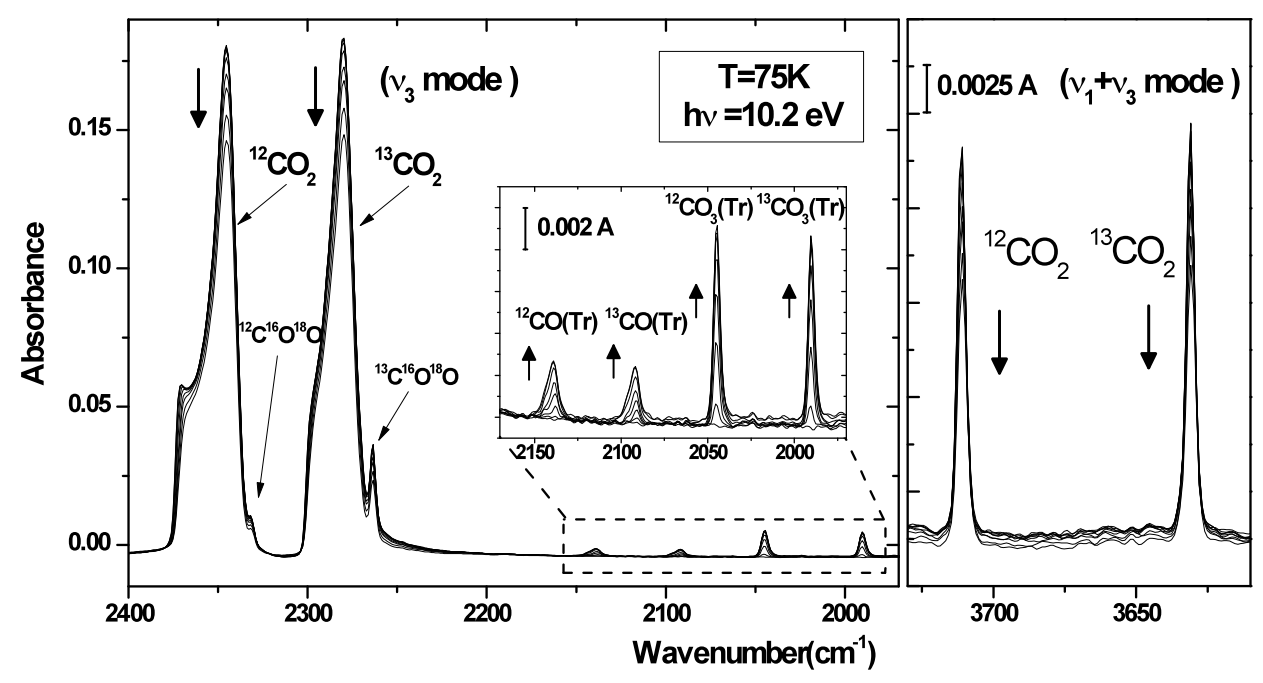

Figure 3.2. Photolysis of $50 \%-50 \%$ isotopomer $\mathrm{CO}_{2}$ (ice) by Lyman-a radiation at $75 \mathrm{~K}$.

It is seen in Figure 3.2 that as depletion of $\mathrm{CO}_{2}$ takes place, $\mathrm{CO}(\mathrm{Tr})$ and $\mathrm{CO}_{3}(\mathrm{Tr})$ species are produced. These species at small concentration exhibit sharp spectral lines, where the peak frequency and line shape for $\mathrm{CO}(\mathrm{Tr})$ and $\mathrm{CO}_{3}(\mathrm{Tr})$ remains nearly constant as their concentrations increase. In Figure 3.3, we use the integrated absorbance of these lines to measure their development in concentration during photochemical production. The rate of production of both ${ }^{12} \mathrm{CO}(\mathrm{Tr})$ and ${ }^{12} \mathrm{CO}_{3}(\mathrm{Tr})$ exceeds that of ${ }^{13} \mathrm{CO}(\mathrm{Tr})$ and ${ }^{13} \mathrm{CO}_{3}(\mathrm{Tr})$ and this rate difference represents a significant kinetic isotope effect. The initial growth of the absorbances has been fitted to an exponential function and the insets to Figure 3.3 show that the initial rate ratios, $\mathrm{m}_{12} / \mathrm{m}_{13}$, measured along the initial growth process, are near 1.13 and 1.12 for the two products, $\mathrm{CO}(\mathrm{Tr})$ and $\mathrm{CO}_{3}(\mathrm{Tr})$. Similar results have also been seen for $a{ }^{12} \mathrm{C}^{16} \mathrm{O}_{2}$ and ${ }^{12} \mathrm{C}^{18} \mathrm{O}_{2}$ mixed ice in the production of isotopomers of $\mathrm{CO}(\mathrm{Tr})$ where ${ }^{18} \mathrm{O}$-labeled products are produced more slowly than ${ }^{16} \mathrm{O}$-labeled products (not shown). 

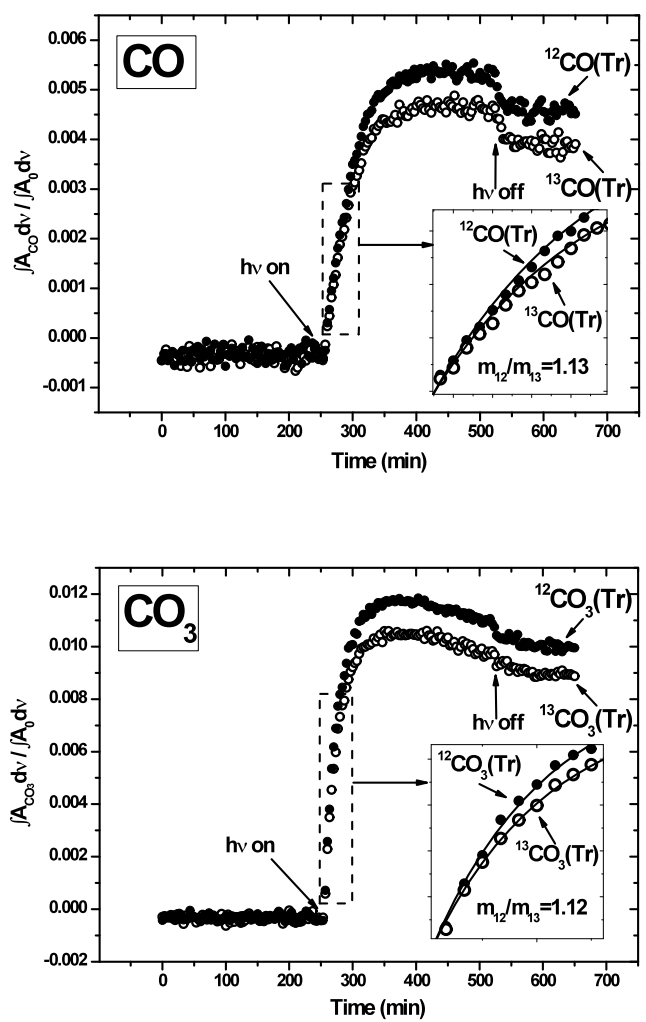

Figure 3.3. $\mathrm{CO}(\mathrm{Tr})$ and $\mathrm{CO}_{3}(\mathrm{Tr})$ product formation from $\mathrm{CO}_{2}(\mathrm{ice})-50 \%-50 \%$.

Figure 3.4 shows that the average integrated absorbance ratios over the entire photochemical process are for $\mathrm{CO}(\mathrm{Tr}),\left\langle\mathrm{A}_{12} / \mathrm{A}_{13}\right\rangle=1.170 \pm 0.007$ and for $\mathrm{CO}_{3}(\mathrm{Tr}),\left\langle\mathrm{A}_{12} / \mathrm{A}_{13}>=1.134 \pm 0.005\right.$ using the small trends in the average to estimate the error. These ratios represent an integration of the photochemical processes over the time of photolysis and resemble the initial rate ratios. As may be seen from Figure 3.3, for both $\mathrm{CO}(\mathrm{Tr})$ and $\mathrm{CO}_{3}(\mathrm{Tr})$, the photochemical production process is accompanied by a depletion process which becomes competitive as the extent of photolysis increases, causing the rate of product growth to come to a maximum and to then decrease. The depletion processes were not investigated and are probably the result of desorption or diffusion of the trapped species out of the radiation-damaged ice or, in the case of $\mathrm{CO}_{3}(\mathrm{Tr})$, its possible photochemical consumption. 
A small correction for the difference in extinction coefficient for trapped isotopomer $\mathrm{CO}$ and $\mathrm{CO}_{3}$ products should be considered. The extinction coefficient should scale as $1 / \mu$, where $\mu$ is the reduced mass associated with the vibrational mode being observed[29]. For $\mathrm{CO}$, this effect should cause the absorbance for ${ }^{12} \mathrm{CO}$ to be increased by $4.9 \%$ compared to ${ }^{13} \mathrm{CO}$; for $\mathrm{CO}_{3}$, the increase should be $5.6 \%$ for ${ }^{12} \mathrm{CO}_{3}$ compared to ${ }^{13} \mathrm{CO}_{3}$. In an attempt to observe the isotopic effect on absorbance, we measured $\varepsilon\left({ }^{12} \mathrm{CO}_{2}\right) / \varepsilon\left({ }^{13} \mathrm{CO}_{2}\right)$ for the $\left(\mathrm{v}_{1}+\mathrm{v}_{3}\right)$ mode of $\mathrm{CO}_{2}$ in the nominal $50 \%-50 \%$ ice. The measured ratio $=0.96 \pm 0.02$ (three separate measurements using peak absorbance), whereas the calculated isotopic effect following the $1 / \mu$ scaling should yield a ratio of 1.057 , with an opposite trend from unity to that measured. On the basis of these calculations and measurements, we estimate a lower value for the corrected $m_{12} / m_{13}$ for CO to be $(1.13-0.05)=1.08$; for $\mathrm{CO}_{3}$, the corrected value of $\mathrm{m}_{12} / \mathrm{m}_{13}=(1.12-0.06)$ $=1.06$. For the purpose of calculations, we employ a ratio of $m_{12} / m_{13}=1.1$ in this work for both molecules.

Figure 3.5 shows measurements of the simultaneous depletion of ${ }^{12} \mathrm{CO}_{2}$ and ${ }^{13} \mathrm{CO}_{2}$ in a $50 \%-50 \%$ mixture of the two isotopomers. The integrated absorbances of $\left(v_{1}+v_{3}\right)$ mode of each isotopomer are normalized to each other at the point where the photochemical decomposition experiments begin. There are three regions in this experiment. In the first region, characterized by the slope $m_{1}$, only $\mathrm{CO}_{2}$ thermal desorption at $75 \mathrm{~K}$ from the ice is observed. In the second region photochemical loss of $\mathrm{CO}_{2}$ is observed in addition to thermal depletion, characterized by the slope $m_{2}$. The decay in region $m_{2}$ is linear with photolysis time. The fractional rate of photochemical depletion is therefore given by $\left(m_{2}-\right.$ $\left.m_{1}\right)$. The third region occurs after Lyman-a irradiation has been interrupted and depletion in this region is governed again by thermal desorption of $\mathrm{CO}_{2}$ after photochemistry ceases. It may be seen that the two isotopomers, ${ }^{12} \mathrm{CO}_{2}$ and ${ }^{13} \mathrm{CO}_{2}$, behave almost identically in all three regions with closely overlapping 
points. The same is observed for $50 \%-50 \%{ }^{12} \mathrm{C}^{16} \mathrm{O}_{2}$ and ${ }^{12} \mathrm{C}^{18} \mathrm{O}_{2}$ (not shown). This lack of isotopic sensitivity for the photochemical depletion of the 50\%-50\% isotopically mixed $\mathrm{CO}_{2}$ ice is expected. A $10 \%$ isotopic difference in rates would yield only $2 \%$ difference in their intensities, which is below our errors in Figure 3.5. Based on the overall change in absorbance in Figure 3.5 from the initial point $\left(\mathrm{A} / \mathrm{A}_{0}=1.00\right)$, about $20 \%$ of the $\mathrm{CO}_{2}$ in the ice has been depleted in the photochemical experiment, when corrected during photodepletion for the extent $(4 \%)$ of thermal desorption.
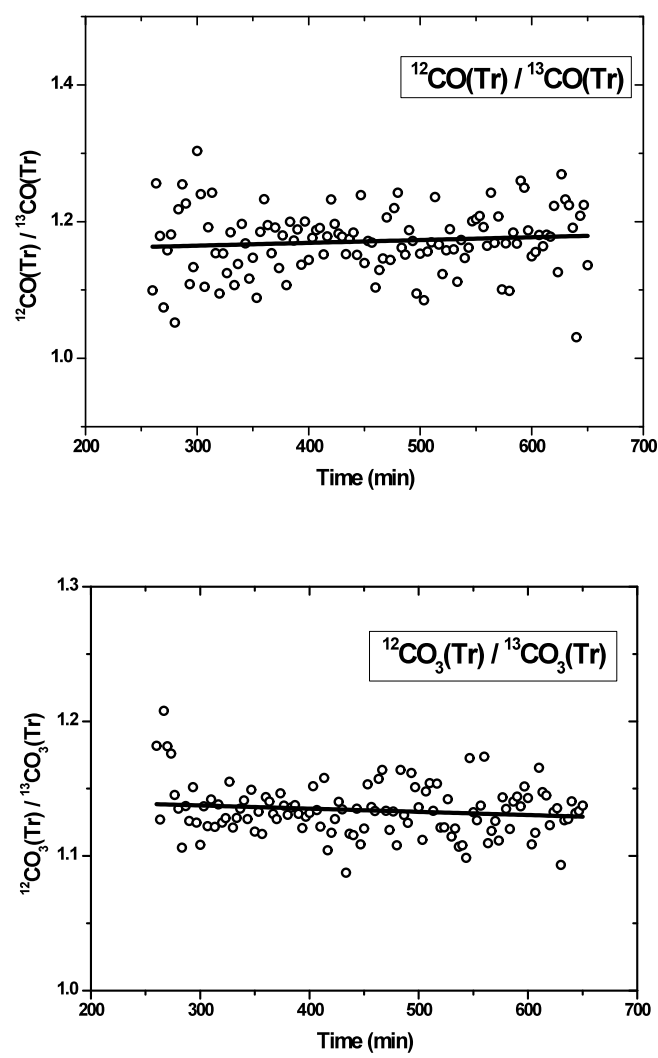

Figure 3.4. Product isotopic ratios during UV-irradiation 


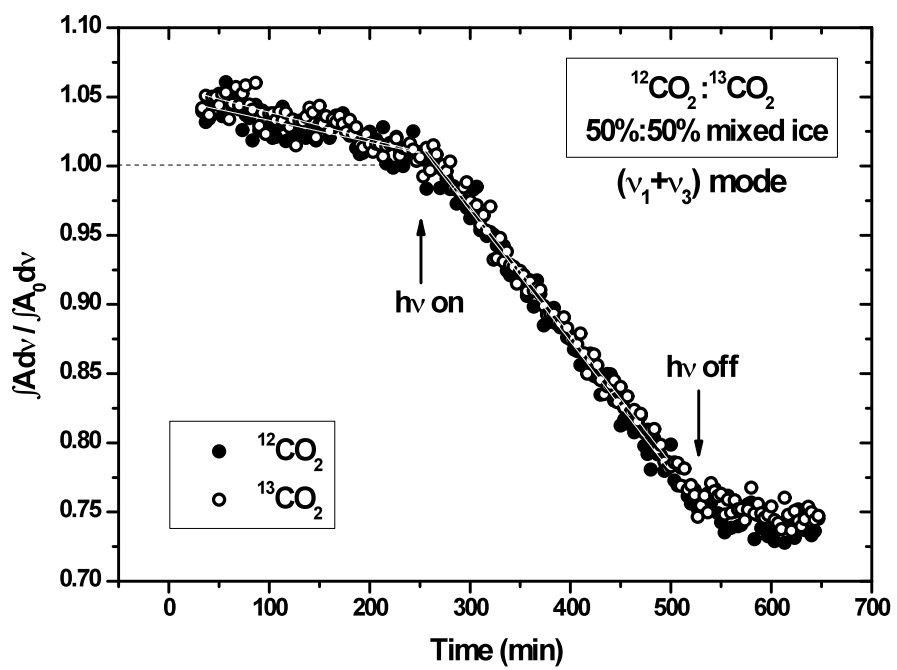

Figure 3.5. Relative rate of depletion of $50 \%-50 \%{ }^{12} \mathrm{CO}_{2}$ and ${ }^{13} \mathrm{CO}_{2}$ by UV-irradiation.

\subsection{Discussion}

\subsubsection{Origin of the Isotope Effect in Photoexcitation - Qualitative}

Figure 3.6 shows a schematic view of the sequence of processes which are thought to occur in $\mathrm{CO}_{2}$ ice upon excitation of the $\mathrm{CO}_{2}$ molecule by Lyman-a radiation. In Figure 3.6a, the $\mathrm{CO}_{2}$ molecule is directly excited by a $10.2 \mathrm{eV}$ photon into an electronically excited state which is repulsive in one $\mathrm{C}-\mathrm{O}$ bond of $\mathrm{CO}_{2}$. With $10.2 \mathrm{eV}$ photon energy, a $\mathrm{CO}_{2}$ molecule is excited from its ground state $\left(\mathrm{X}^{1} \Sigma_{\mathrm{g}}^{+}\right)$to ${ }^{1} \Pi_{\mathrm{g}},{ }^{1} \Sigma_{\mathrm{u}}$, or ${ }^{1} \Delta_{\mathrm{u}}$ excitation states, and yields energetic photodissociation products $\mathrm{CO}\left(\mathrm{X}^{1} \Sigma_{\mathrm{g}}^{+}\right)$and $\mathrm{O}\left({ }^{3} \mathrm{P},{ }^{1} \mathrm{D},{ }^{1} \mathrm{~S}\right)[30,31]$. This electronic excitation process may be described as a Franck-Condon transition to the excited potential energy surface and is followed by $\mathrm{C}-\mathrm{O}$ bond lengthening producing rapid nuclear motion. During this period, there is a probability that the excess electronic potential energy of the molecule in its $\mathrm{CO}_{2}$ (ice) cage will drain into the $\mathrm{CO}_{2}$ ice lattice (shown as $\Delta \mathrm{E}^{\star}$ ). Such a quenching process will return the dissociating $\mathrm{CO}_{2}$ molecule to its lower potential energy state and interrupt the 
bond dissociation process. The quenching probability will be smaller for the faster-moving ${ }^{12} \mathrm{CO}$ fragment and it will therefore have a higher probability of escape from the cage where it was formed to a nearby lattice site. Thus, the rate of formation of ${ }^{12} \mathrm{CO}(\mathrm{Tr})$ will be larger than ${ }^{13} \mathrm{CO}(\mathrm{Tr})$, as observed.

The formation of $\mathrm{CO}_{3}(\mathrm{Tr})$ with a kinetic preference for ${ }^{12} \mathrm{CO}_{3}(\mathrm{Tr})$ formation may be understood in Figure 3.6a as follows: By diffusion in the $\mathrm{CO}_{2}$ matrix, a $\mathrm{CO}$ product molecule and an $\mathrm{O}$ product atom encounter each other in an exothermic reaction to produce vibrationally-excited $\mathrm{CO}_{2}\left(\mathrm{~V}^{*}\right)$. The subsequent reaction of $\mathrm{CO}_{2}\left(\mathrm{~V}^{*}\right)$ with a second $\mathrm{O}(\mathrm{Tr})$ will be rapid and highly exothermic. Therefore the excess of ${ }^{12} \mathrm{CO}(\mathrm{Tr})$ originally produced in the photochemical decomposition will efficiently convert to an excess of ${ }^{12} \mathrm{CO}_{3}(\mathrm{Tr})$ and the original kinetic preference for ${ }^{12} \mathrm{CO}(\mathrm{Tr})$ formation will therefore be rapidly passed onto ${ }^{12} \mathrm{CO}_{3}(\mathrm{Tr})$. It is noted (Figure 3.3) that the relative isotope formation rates for ${ }^{12} \mathrm{C}$-species $/{ }^{13} \mathrm{C}$-species is nearly the same $(\sim 1.1)$ for both $\mathrm{CO}$ and $\mathrm{CO}_{3}$, indicating that the preference for the generation of the lighter isotopomer of $\mathrm{CO}_{3}$ is due to the original isotope effect producing an excess of ${ }^{12} \mathrm{CO}$ from $\mathrm{CO}_{2}{ }^{*}$. The postulate that two sequential reactions involving $\mathrm{O}(\mathrm{Tr})$ are necessary for $\mathrm{CO}_{3}(\mathrm{Tr})$ formation is supported by our observation of the formation of traces of $\mathrm{O}_{3}(\mathrm{Tr})$ which also must require multiple elementary steps involving $\mathrm{O}(\mathrm{Tr})$ species in the $\mathrm{CO}_{2}$ matrix. In another experiment (not shown), when the $\mathrm{CO}_{2}$ molecules are diluted in a $\mathrm{Xe}$ matrix $\left({ }^{12} \mathrm{CO}_{2}:{ }^{13} \mathrm{CO}_{2}: \mathrm{Xe}=1: 1: 8\right)$, little or no preference of ${ }^{12} \mathrm{CO}_{3}$ production is observed during photolysis, possibly because $\mathrm{CO}_{2}\left(\mathrm{~V}^{*}\right)$ produced by $\mathrm{CO}+\mathrm{O}$ recombination in the Xe-diluted matrix more extensively relaxes during its diffusion in the predominantly Xe lattice before encountering an $\mathrm{O}(\mathrm{Tr})$ molecule. As shown in Figure 3.6, the $\mathrm{CO}_{2}$ desorption process, $k_{d}$ occurs also from $\mathrm{CO}_{2}\left(\mathrm{~V}^{\star}\right)$ by a vibration-translation energy exchange process. In addition, $\mathrm{CO}(\mathrm{g})$ escapes from $\mathrm{CO}+\mathrm{O}$ following the primary $\mathrm{C}-\mathrm{O}$ bond dissociation process. 

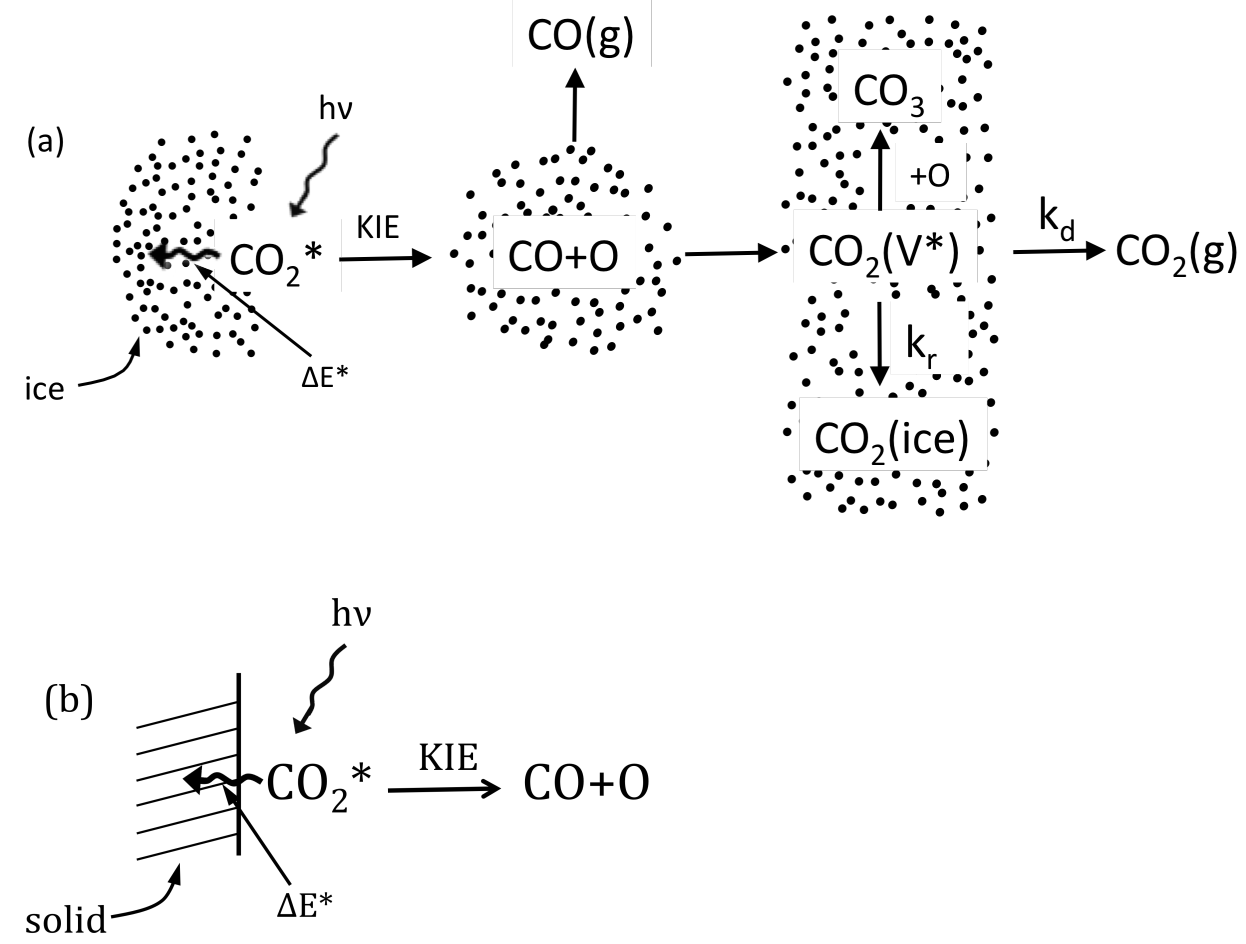

Figure 3.6. Comparison of photoexcitation of $\mathrm{CO}_{2}$ (ice) and adsorbed $\mathrm{CO}_{2}$ on surfaces. The matrix of the $\mathrm{CO}_{2}$ (ice) is represented by dots. In (a), the $\mathrm{CO}_{2}$ (ice) matrix participates in quenching the electronically-excited $\mathrm{CO}_{2}{ }^{*}$. In (b), the solid surface participates in quenching the adsorbed molecule following electronic excitation.

\subsubsection{Detailed Considerations of the Origin of the Isotope Effect- Connection to the Menzel-Gomer-Redhead Model of Electron Stimulated Desorption from Surfaces.}

The enrichment of ${ }^{12} \mathrm{CO}$ by the isotopic separation effect on the upper state is analogous to a well-known isotopic enrichment effect which occurs on surfaces where an adsorbed molecule is electronically excited. This is the Menzel-GomerRedhead (MGR) effect [13-15] schematically shown in Figure 3.6b. Here an adsorbed electronically-excited species liberates low mass isotopic ionic or neutral fragments faster than high mass fragments due to the preferential highmass surface quenching (or ion neutralization) effect influencing the excited species during its dissociation. For chemisorbed ${ }^{16} \mathrm{O} /{ }^{18} \mathrm{O}$ on a $\mathrm{W}(100)$ surface, 
the ${ }^{16} \mathrm{O}^{+} /{ }^{18} \mathrm{O}^{+}$ratio is $1.47 \pm 0.10$. The ratio of ${ }^{16} \mathrm{O} /{ }^{18} \mathrm{O}$ neutral desorption rates was found to be near 1.06 in the same study[17].

The preferential isotope quenching effect may be understood in more detail as shown below and in Figure 3.7. The total probability of molecular fragmentation of a chemical bond undergoing repulsive dissociation (bond breaking) on an excited state potential curve is given as $\mathrm{P}_{\mathrm{BB}}$.

$$
P_{B B}=\exp \left(-\int_{r_{0}}^{r_{c}} \frac{d r}{\tau v}\right)
$$

where $\tau$ is the distance-dependent lifetime of the bond and $v$ is the velocity of the isotopically-labeled fragment. The starting point of excitation is $r_{0}$. The limiting point for recapture and quenching is $r_{c}$, beyond which bond breaking will occur, leading to reaction.

The velocity of the photo-fragment $v$ at separation, $r$, may be expressed in terms of the potential energy lost,

$$
v=\left\{\frac{2}{m}\left[V\left(r_{0}\right)-V(r)\right]\right\}^{1 / 2}
$$

giving

$$
P_{B B}=\exp \left[-\left(\frac{m}{2}\right)^{1 / 2} \int_{r_{0}}^{r_{c}} \frac{d r}{\tau \sqrt{V\left(r_{0}\right)-V(r)}}\right]
$$

where the dependence on isotopic mass is introduced. The use of $\mathrm{m}$ rather than the reduced-mass for the dissociating $\mathrm{O} \cdots \mathrm{CO}$ bond assumes that the $\mathrm{O}$ atom is efficiently coupled, dynamically to the ice.

The total cross section for the breaking of an O‥CO bond is given by

$$
\sigma_{B B}=P_{B B} \cdot \sigma_{\text {excitation }}
$$

where $\sigma_{\text {excitation }}$ is the electronic excitation cross section. 
For chemical bond breaking, isotopic mass particles will experience almost identical repulsive potentials, $\mathrm{V}(\mathrm{r})$, and identical excitation cross sections,

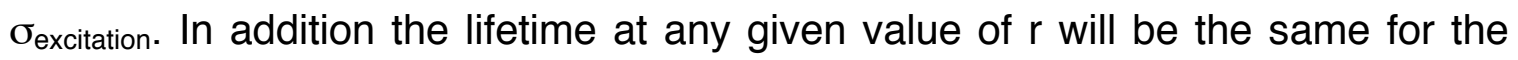
two isotopic masses.

From equations (3) and (4), therefore

$$
\frac{\sigma_{\text {light }}}{\sigma_{\text {heavy }}}=\exp \left\{\left[\left(m_{\text {heavy }}\right)^{1 / 2}-\left(m_{\text {light }}\right)^{1 / 2}\right] \cdot C\right\}
$$

where $C$ is a constant and $C=\frac{1}{\sqrt{2}} \int_{r_{0}}^{r_{c}} \frac{d r}{\tau \sqrt{V\left(r_{0}\right)-V(r)}}$.

Equation (5) may be rearranged to give :

$$
\frac{\sigma_{\text {light }}}{\sigma_{\text {heavy }}}=\left(\frac{\sigma_{\text {excitation }}}{\sigma_{\text {light }}}\right)^{\left[\sqrt{m_{\text {heayy }} / m_{\text {light }}}-1\right]}=\left(\frac{1}{P_{B B}}\right)^{\left[\sqrt{m_{\text {heavy }} / m_{\text {light }}}-1\right]}
$$

It may be seen from equation (6) that $\sigma_{\text {light }} / \sigma_{\text {heavy }}$ will depend on $1 / \mathrm{P}_{\mathrm{BB}}$. For a given mass ratio, the isotope effect, $\sigma_{\text {light }} / \sigma_{\text {heavy, }}$, will increase as $\mathrm{P}_{\mathrm{BB}}$ becomes smaller.

This formalism relating equation (5) to equation (6) was used in the work of Wolf et al. and Zhu et al.[32, 33] as applied to the isotopic fractionation of adsorbed $\mathrm{H}_{2} \mathrm{O} / \mathrm{D}_{2} \mathrm{O}$ on $\mathrm{Pd}(111)$, and is similar to earlier treatment[15, 17]. Here cross sections favoring $\mathrm{H}$-labeled species are about a factor of 2 greater than for D-labeled species in both photo-desorption and photo-dissociation of absorbed water molecules from $\mathrm{Pd}(111)$.

\subsubsection{Overall Kinetic Model for Photodissociation and Photodesorption of $\mathrm{CO}_{2}$ ice.}

Figure 3.7 schematically shows a pair of potential energy curves for a $\mathrm{CO}_{2}$ (ice) molecule and the electronically excited $\mathrm{CO}_{2}{ }^{*}$ state. Excitation by Lyman- 
a radiation leads to more rapid ${ }^{12} \mathrm{CO}_{2}$ dissociation than ${ }^{13} \mathrm{CO}_{2}$ dissociation on the upper curve. During $\mathrm{C}-\mathrm{O}$ bond dissociation, quenching will often return the $\mathrm{CO}_{2}{ }^{*}$ molecule to very highly vibrationally-excited states on the ground state curve. From this point the $\mathrm{CO}_{2}$ molecule can desorb (process $\mathrm{k}_{\mathrm{d}}$ ) or it can further relax vibrationally in the ice lattice (process $\mathrm{k}_{\mathrm{r}}$ ). On the upper state curve, evolution to larger $r_{\text {Co-o }}$ distances (beyond $r_{c}$ ) results in $\mathrm{CO}(\mathrm{Tr}$ ) production and in formation of $\mathrm{CO}_{3}(\mathrm{Tr})$ in the reaction channel, both species being trapped in the $\mathrm{CO}_{2}$ matrix. In addition, $\mathrm{CO}(\mathrm{Tr})$ can also desorb as $\mathrm{CO}(\mathrm{g})$.

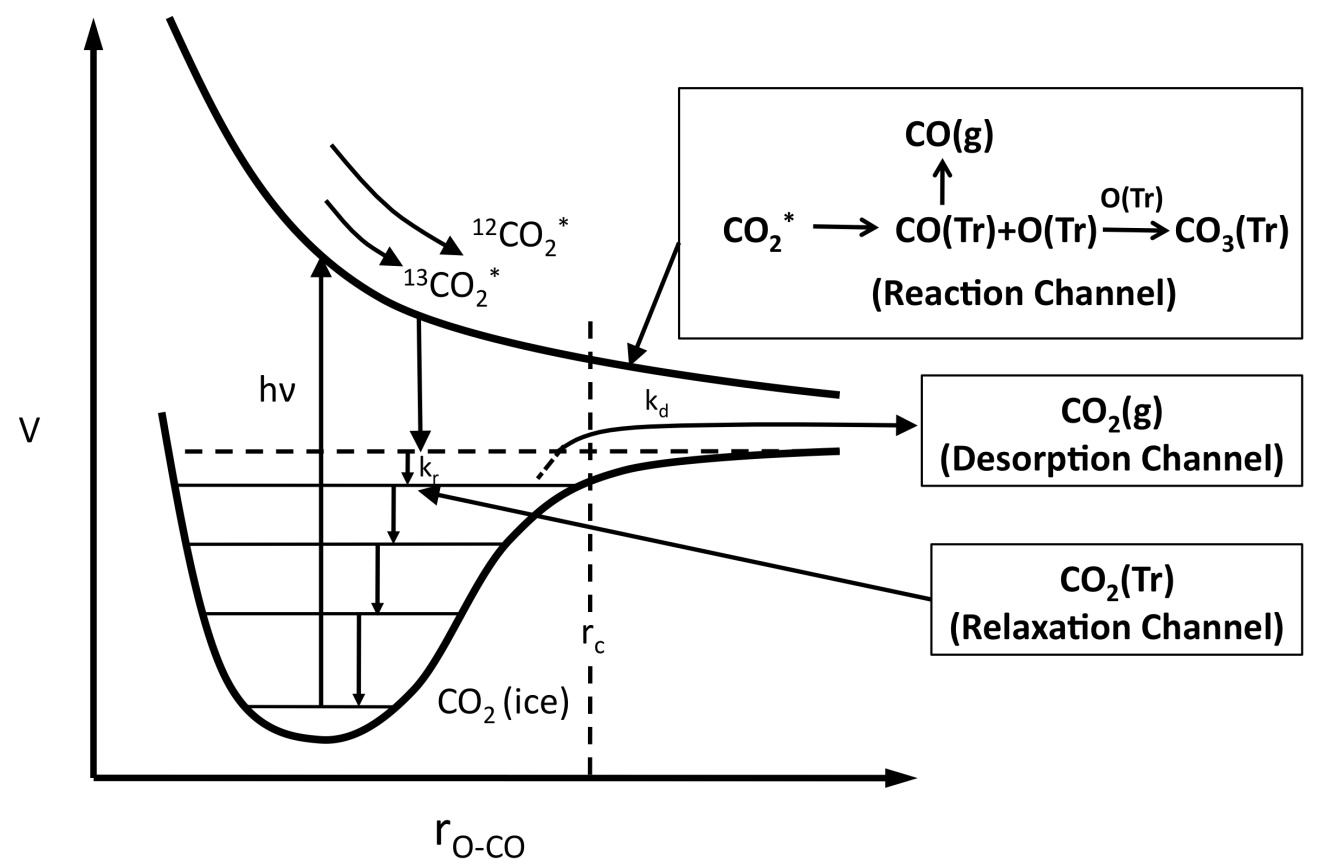

Figure 3.7. Reaction, desorption and vibrational relaxation channels in $\mathrm{CO}_{2}$ (ice) excited by UV-irradiation

The rate of loss of $\mathrm{CO}_{2}$ by Lyman-a excitation is, to within experimental error, the same for each isotopomer of $\mathrm{CO}_{2}$, as shown in Figure 3.5, when a 50\%-50\% ratio of ${ }^{12} \mathrm{CO}_{2}{ }^{-13} \mathrm{CO}_{2}$ is present in the matrix. The ${ }^{12} \mathrm{CO}_{2} /{ }^{13} \mathrm{CO}_{2}$ isotope effect is not observable in desorption since it is fractionally small compared to the concentration of $\mathrm{CO}_{2}$ (ice). 


\subsubsection{Efficiency of Photodesorption from $\mathrm{CO}_{2}$ ice at $75 \mathrm{~K}$ by Lyman-a} Radiation

As shown in Figure 3.5, the $\mathrm{CO}_{2}$ loss due to photo-excitation is approximately $20 \%$ during the 260 minutes of Lyman- $\alpha$ irradiation time. Using the lamp photon flux of $2.7 \times 10^{14}$ photons $\cdot \mathrm{cm}^{-2} \cdot \mathrm{s}^{-1}$, and the ice thickness of $50 \mathrm{~nm}$, we estimate the efficiency of photodepletion as $\sim 8.4 \times 10^{-3} \mathrm{CO}_{2}$ /photon, corresponding to a $\mathrm{CO}_{2}$ depletion cross section of $7.3 \times 10^{-20} \mathrm{~cm}^{2}$ for a $50 \%-50 \%{ }^{12} \mathrm{CO}_{2^{-}}{ }^{13} \mathrm{CO}_{2}$ mixed ice. For a pure ${ }^{12} \mathrm{CO}_{2}$ or ${ }^{13} \mathrm{CO}_{2}$ ice, we find that this photodepletion cross section decreases to $\sigma_{\text {depletion }} \sim 5 \times 10^{-20} \mathrm{~cm}^{2}$, corresponding to $\sim 6 \times 10^{-3} \mathrm{CO}_{2} /$ photon. This value is consistent with the experimental measurement by Oberg et al.[5], where they obtain $2.3 \times 10^{-3} \mathrm{CO}_{2}$ /photon at $18 \mathrm{~K}$. It should be noted that the photodesorption rate, $\mathrm{k}_{\mathrm{d}}$, of the $\mathrm{CO}_{2}$ molecule is highly dependent on the composition of the surrounding molecules in the ice, because of differing lattice relaxation efficiency for the vibrationally hot $\mathrm{CO}_{2}\left(\mathrm{~V}^{*}\right)$ molecule. The detuning of vibrational modes between $\mathrm{CO}_{2}\left(\mathrm{~V}^{*}\right)$ and neighboring lattice molecules will result in an increase in the photodesorption rate, $k_{d}$, as vibrational relaxation in the lattice diminishes. The details of the lattice relaxation effect for $\mathrm{CO}_{2}$ lattices are discussed in a following paper[34].

It is of interest to employ equation (6) to understand the overall efficiencies of the photo-induced processes in the ice. From equation (6), the probability of $\mathrm{O} \cdots \mathrm{CO}$ bond breaking $\left(\mathrm{P}_{\mathrm{BB}}\right)$ may be found. Assuming that the escaping fragment, $\mathrm{CO}$, determines the dynamics on the upper state curve, and that for the photoreaction processes, $\sigma_{\text {light }} / \sigma_{\text {heavy }}=1.1$ from the data in Figure 3.3 , we obtain $\mathrm{P}_{\mathrm{BB}}=$ 0.0046 . This implies that $\sigma_{\text {excitation }} / \sigma_{\text {light }}=218$. Therefore for every $\sim 200$ excitation events for a $\mathrm{CO}_{2}$ (ice) molecule, one reactive event occurs involving the breaking of a O‥CO bond and the formation of a trapped reaction product molecule and $99.5 \%$ of the excitation events transfer the $\mathrm{CO}_{2}\left(\mathrm{~V}^{*}\right)$ molecules back to the $\mathrm{CO}_{2}$ lattice. Since every excited $\mathrm{CO}_{2}$ molecule must either react, desorb, or return to 
the lattice as an unreacted $\mathrm{CO}_{2}$ molecule, we see that the reaction process is a minor $(0.5 \%)$ process compared to the recycling of $\mathrm{CO}_{2}$ in the ice by vibrational relaxation in the ice matrix.

Similar conclusions have been reached by Oberg, et al.[5]. They report that the reaction channel producing $\mathrm{CO}_{3}$ cannot exceed $5 \%$ of the desorption channel at $18 \mathrm{~K}$ and thus, in agreement with this work, reaction must be considered to be a minor process in the ice.

\subsubsection{Summary of Relative Cross Sections for All Processes}

The data obtained in this work allow an estimate to be made for all of the cross sections for the major photochemical processes operating when $\mathrm{CO}_{2}$ (ice) is irradiated by Lyman-a radiation at $75 \mathrm{~K}$. The quantum yield for the $10.2 \mathrm{eV}$ excitation of the gas phase process, $\mathrm{CO}_{2}+\mathrm{hv} \rightarrow \mathrm{CO}+\mathrm{O}$, has been measured [35] to be close to 1 . This allows the prediction of the excitation cross section from the $\mathrm{CO}_{2}$ absorption cross section of $\sigma_{\text {absorption }}=9 \times 10^{-18} \mathrm{~cm}^{2}$. Since, based on the isotope effect, $P_{\mathrm{BB}}$ from the excited state is $4.6 \times 10^{-3}$, the cross section for reaction is $9 \times 10^{-18} \mathrm{~cm}^{2} \times 4.6 \times 10^{-3}=4 \times 10^{-20} \mathrm{~cm}^{2}$. This is close to the measured cross section $\left(7.3 \times 10^{-20} \mathrm{~cm}^{2}\right.$ ) (Figure 3.5) for depletion of the $50 \%-50 \%$ mixed $\mathrm{CO}_{2}$ ice. We also find by mass spectrometric studies of the gas phase composition during Lyman- $\alpha$ irradiation that $\mathrm{CO}$ desorption is much faster than $\mathrm{CO}_{2}$ desorption, as also observed by Bahr et al. [36]. We could also use the final integrated absorbance of the $\mathrm{CO}(\mathrm{Tr})$ species $\left(\sim 0.020 \mathrm{~cm}^{-1}\right)$ and the extinction coefficient $\varepsilon=2.6 \times 10^{6} \mathrm{~cm} \cdot \mathrm{mol}^{-1}$ [37] to estimate that $4.6 \times 10^{15} \mathrm{CO}$ molecules per $\mathrm{cm}^{2}$ are trapped after photodissociation is complete. This corresponds to $\sigma_{\text {reaction }}=$ $3 \times 10^{-20} \mathrm{~cm}^{2}$ for $\mathrm{CO}(\mathrm{Tr})$ production. Since some $\mathrm{CO}(\mathrm{Tr})$ has desorbed at the end

of the photoreaction period (see Figure 3.3) we consider this estimation of $\sigma_{\text {reaction }}$ which is determined independently from $\mathrm{P}_{\mathrm{BB}}$ to be reasonable in comparison to $\sigma_{\text {reaction }}=4 \times 10^{-20} \mathrm{~cm}^{2}$ as determined by the isotope effect. 
Since we have an estimate of $\sigma_{\text {excitation }}=9 \times 10^{-18} \mathrm{~cm}^{2}$ as well as $\sigma_{\text {depletion }}=$ $7.3 \times 10^{-20} \mathrm{~cm}^{2}$, by difference we obtain $\sigma_{\text {relaxation }}=8.9 \times 10^{-18} \mathrm{~cm}^{2}$. The cross sections for all processes are listed below in Table 3.2:

Table 3.2. Cross Sections for Lyman-a Induced Photoprocesses in $50 \%-50 \%{ }^{12} \mathrm{CO}_{2}-$ ${ }^{13} \mathrm{CO}_{2}$ (ice) at $75 \mathrm{~K}$

\begin{tabular}{|c|c|c|}
\hline Process & Cross section $\left(\mathrm{cm}^{2}\right)$ & Notes and references \\
\hline$\sigma_{\text {absorption }}$ & $9 \times 10^{-18}$ & This work \\
\hline$\sigma_{\text {excitation }}$ & $9 \times 10^{-18}$ & $\begin{array}{l}\text { Based on quantum yield in gas phase } \\
\text { [35] }\end{array}$ \\
\hline$\sigma_{\text {depletion }}$ & $7.3 \times 10^{-20}$ & This work \\
\hline$\sigma_{\text {reaction }}$ & $4 \times 10^{-20} ; 3 \times 10^{-20}$ (for CO) & $\begin{array}{l}\text { This work (from isotope effect); (from } \\
\left.\int A_{c o d} d v\right)\end{array}$ \\
\hline$\sigma_{\text {desorption }}$ & $3.3 \times 10^{-20}$ & This work \\
\hline$\sigma_{\text {relaxation }}$ & $8.9 \times 10^{-18}$ & This work \\
\hline
\end{tabular}

A schematic diagram of the contribution of all of the processes leading to reaction, desorption and relaxation of Lyman-a excited $\mathrm{CO}_{2}$ ice is shown in Figure 3.8, based on the cross sections listed in Table 3.2. 


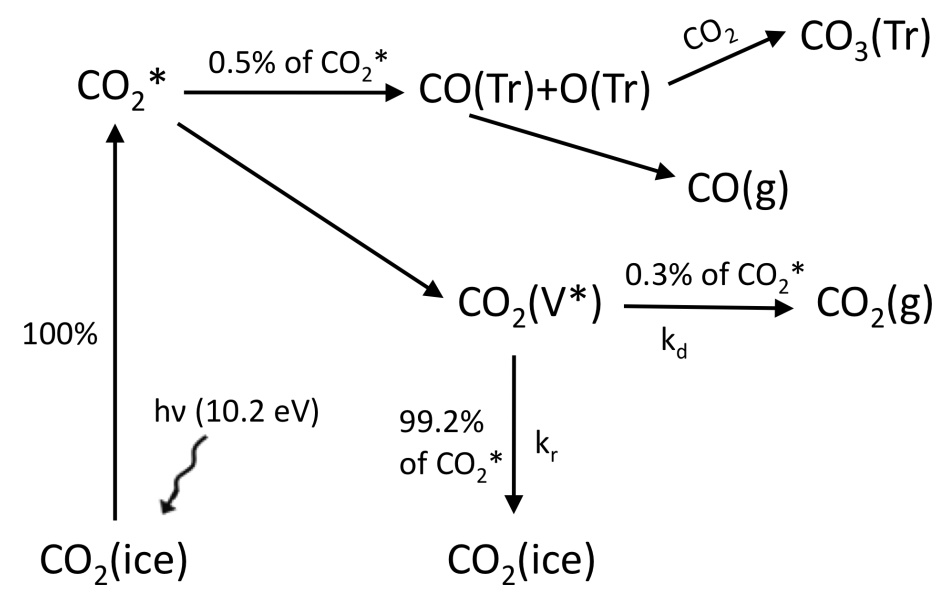

Figure 3.8. Schematic diagram of $\mathrm{CO}_{2}$ photoprocesses for Lyman- $\alpha$ excitation of $\mathrm{CO}_{2}$ (ice).

\subsection{Summary}

We have employed Lyman-a radiation and transmission IR spectroscopy to measure all of the main photoprocesses which occur in a $50 \mathrm{~nm}$ thick film of $\mathrm{CO}_{2}$ (ice) at $75 \mathrm{~K}$. The following phenomena have been observed:

1. The photodecomposition and photodesorption of $\mathrm{CO}_{2}$ (ice) have been observed to occur simultaneously. Photodecomposition yields trapped-CO species and trapped- $\mathrm{CO}_{3}$ species in the $\mathrm{CO}_{2}$ matrix.

2. About $15 \%$ of the $50 \mathrm{~nm} \mathrm{CO}_{2}$ film is photodepleted in $1.6 \times 10^{4} \mathrm{~s}$ irradiation time, with a yield of $6 \times 10^{-3} \mathrm{CO}_{2} /$ photon for a pure ${ }^{12} \mathrm{CO}_{2}$ ice, which is essentially constant throughout the time period of the experiment.

3. A significant ${ }^{12} \mathrm{C} /{ }^{13} \mathrm{C}$ isotope effect is observed in the photochemical rate of formation of both $\mathrm{CO}$ and $\mathrm{CO}_{3}$ species. We measure for the photo-reaction process that $\sigma_{\text {light }} / \sigma_{\text {heavy }} \cong 1.1$ for both reactions. The cross section for the reaction process for the production of $\mathrm{CO}$ and $\mathrm{CO}_{3}$ photoproducts is a minor one, and corresponds to $\sim 0.5 \%$ conversion of electronically-excited $\mathrm{CO}_{2}{ }^{*}$ to $\mathrm{CO}+\mathrm{O}$. The 
cross section for the reaction process for $\mathrm{CO}_{2}$ desorption is $\sigma_{\text {desorption }}=3.3 \times 10^{-20}$ $\mathrm{cm}^{2}$. This corresponds to $\sim 0.3 \%$ conversion of electronically-excited $\mathrm{CO}_{2}{ }^{*}$ to $\mathrm{CO}_{2}(\mathrm{~g})$.

4. The isotope effect seen in the reaction process is caused by the different quenching rates for the bond dissociation process, $\mathrm{CO}_{2}+\mathrm{hv} \rightarrow \mathrm{CO}_{2}{ }^{*} \rightarrow \mathrm{CO}+\mathrm{O}$, where all species are confined in the $\mathrm{CO}_{2}$ ice matrix. Electronic quenching of the $\mathrm{CO}_{2}{ }^{*}$ state by its interaction with the $\mathrm{CO}_{2}$ matrix involves quenching rates which are smaller for the light isotopic particle escaping from the breaking bond, and resembles similar isotopically-governed quenching processes for electronicallyexcited chemisorbed molecules on surfaces.

5. Measurements of the isotope effect for the photo-reaction processes yielding $\mathrm{CO}$ and $\mathrm{CO}_{3}$ allow all of the cross sections for excitation, desorption, reaction, and vibrational relaxation in the $\mathrm{CO}_{2}$ lattice to be evaluated. The measurement of the isotope effect allows $\sigma_{\text {reaction }}=4 \times 10^{-20} \mathrm{~cm}^{2}$ to be determined without knowing the IR extinction coefficient of a photochemical product.

6. The reaction between $\mathrm{CO}$ species and $\mathrm{O}$ species trapped in the $\mathrm{CO}_{2}$ ice lattice occurs by recombination to produce vibrationally-excited $\mathrm{CO}_{2}$ which quickly reacts with additional trapped $\mathrm{O}$ species to produce trapped $\mathrm{CO}_{3}$. This allows the isotope effect causing the preferential production of ${ }^{12} \mathrm{CO}$ to govern the incorporation of excess ${ }^{12} \mathrm{CO}$ into ${ }^{12} \mathrm{CO}_{3}$.

7. The observation of a significant ${ }^{12} \mathrm{C} /{ }^{13} \mathrm{C}$ isotope effect in the Lyman- $\alpha$ induced photodecomposition of $\mathrm{CO}_{2}$ in an ice environment suggests that in interstellar chemistry, where bond-breaking photo-processes are operative in ices, such processes can significantly influence the isotopic composition of reaction product species. Transfer of isotopically enriched species in ices to the gas phase during warming followed by condensation elsewhere can occur repeatedly over astronomical times. Hence the historical information derived from 
spectroscopically-determined isotopic ratios in space may be distorted by this effect due to isotopic fractionation during photochemical bond breaking in ices. In general, based on the model employed and our experimental results, it is expected that such photodissociative isotopic enrichment effects will favor the production of the light isotopomer reaction products.

\subsection{References:}

1. Pimentel, G.C., Formation and Trapping of Free Radicals, 1960, New York, Academic Press Inc.

2. Andrews, L., Infrared Spectra of Free Radicals and Chemical Intermediates in Inert Matrices. Annual Review of Physical Chemistry, 1971. 22: p. 109.

3. Watanabe, N. and A. Kouchi, Ice surface reactions: A key to chemical evolution in space. Prog. Surf. Sci., 2008. 83: p. 439.

4. Viti, S., et al., Evaporation of ices near massive stars: models based on laboratory temperature programmed desorption data. Mon. Not. R. Astron. Soc. , 2004. 354: p. 1141.

5. Oberg, K.I., E.F. van Dishoeck, and H. Linnartz, Photodesorption of ices I: CO, $\mathrm{N}_{2}$, and $\mathrm{CO}_{2}$. Astron. Astrophys., 2009. 496: p. 281.

6. Fedorova, A.A., et al., Remote sounding of the Martian dust from ISO

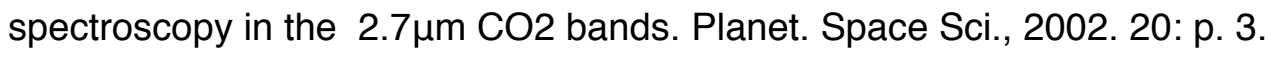

7. Fraser, H.J., M.R.S. McCoustra, and D.A. Williams, The molecular universe. Astron. Geophys., 2002. 43: p. 2.10.

8. Ehrenfreud, P. and W.A. Schutte, Astrochemistry - From Molecular Clouds to Planetary Systems2000, San Francisco: ASP Publishing.

9. Lis, D.C., et al., Nitrogen isotopic fractionation in interstellar ammonia. Astrophys. J., 2010. 710: p. L49.

10. Rubin, R.H., et al., $12 \mathrm{C} / 13 \mathrm{C}$ ratio in planetary nebulae from the iue archives. Astrophys. J., 2004. 605: p. 784.

11. Vanýsek, V. and J. Rahe, The12C/13C isotope ratio in comets, stars and interstellar matter. Earth, Moon, and Planets, 1978. 18(4): p. 441. 
12. Wyckoff, S., et al., The C-12/C-13 abundance ratio in Comet Halley. Astrophys. J., 1989. 339: p. 488.

13. Menzel, D. and R. Gomer, Desorption from Metal Surfaces by Low-Energy Electrons. J. Chem. Phys., 1964. 41: p. 3311.

14. Redhead, P.A., Interaction of Slow Electrons with Chemisorbed Oxygen. Can. J. Phys., 1964. 42: p. 886.

15. Ramsier, R.D. and J.T. Yates, Jr., Electron-stimulated desorption: Principles and applications. Surface Science Reports, 1991. 12: p. 246.

16. Zhu, X.Y. and J.M. White, Vibration-Mediated UV Photodesorption: Ammonia on GaAs. Phys. Rev. Lett., 1992. 68(22): p. 3359.

17. Madey, T.E., et al., Isotope Effect in Electron Stimulated Desorption: Oxygen Chemisorbed on Tungsten. J. Chem. Phys. , 1970. 52: p. 5215.

18. Lakowicz, J.R., Principles of Fluorescence Spectroscopy, 3rd Edition, 2006: Springer.

19. Rajappan, M., C. Yuan, and J.T. Yates, Jr., Lyman-a driven molecule formation on $\mathrm{SiO}_{2}$ surfaces - connection to astrochemistry on dust grains in the interstellar medium. J. Chem. Phys., 2011. 134: p. 064315.

20. Rajappan, M., et al., Photochemical Decomposition of $\mathrm{N}_{2} \mathrm{O}$ by Lyman-a Radiation: Scientific Basis for a Chemical Actinometer. J. Phys. Chem. A, 2010. 114: p. 3443.

21. Xu, J., H.J. Jansch, and J.T. Yates, Jr., Cryogenic trick for enhanced cooling using liquid nitrogen. J. Vac. Sci. Technol. A, 1993. 11: p. 726.

22. Gerakines, P.A., et al., The infrared band strength of $\mathrm{H}_{2} \mathrm{O}, \mathrm{CO}$ and $\mathrm{CO}_{2}$ in laboratory simulations of astrophysical ice mixtures. Astron. Astrophys., 1995. 296: p. 810.

23. Mason, N.J., et al., VUV spectroscopy and photo-processing of astrochemical ices: an experimental study. Faraday Discuss., 2006. 133: p. 311.

24. Warren, S.G., Optical constants of carbon dioxide ice. Applied Optics,, 1986. 25: p. 2650.

25. Ovchinnikov, M.A. and C.A. Wight, Inhomogeneous broadening of infrared and Raman spectral bands of amorphous and polycrystalline thin films. J. Chem. Phys., 1993. 99: p. 3374.

26. Gerakines, P.A., W.A. Schutte, and P. Ehrenfreund, Ultraviolet processing of interstellar ice analogs. I. Pure ices. Astron. Astrophys., 1996. 312: p. 289. 
27. Moll, N.G., D.R. Clutter, and W.E. Thompson, Carbon trioxide: its production, infrared spectrum, and structure studied in a matrix of solid $\mathrm{CO}_{2}$. J. Chem. Phys., 1966. 45: p. 4469.

28. Bennett, C.J., et al., Untangling the formation of the cyclic carbon trioxide isomer in low temperature carbon dioxide ices. Phys. Chem. Chem. Phys., 2004. 6: p. 735.

29. Singh, S., Infrared Band Intensities: Transition Moment Matrix Eiements For Fundamentals And Overtones, Part III. Isotope effect. J. Molec. Structure, 1985. 127: p. 203.

30. Knowles, P.J. and P. Rosmus, On the assignment of the electronically excited single tstates in linear $\mathrm{CO}_{2}$. Chem. Phys. Lett., 1988. 146: p. 230.

31. Okabe, H., Photochemistry of Small Molecules1978: John Wiley \& Sons Inc.

32. Wolf, M., et al., Dynamics of The Ultraviolet Photochemistry of Water Adsorbed on Pd(111). J. Chem. Phys., 1991. 94: p. 4609.

33. Zhu, X.Y., et al., Laser induced interaction of ammonia with GaAs(100). II. Desorption dynamics. J. Chem. Phys., 1992. 97: p. 5868.

34. Yuan, C. and J.T. Yates, Jr., Lyman-a Photodesorption from $\mathrm{CO}_{2}$ (ice) at $75 \mathrm{~K}$ : Role of $\mathrm{CO}_{2}$ Vibrational Relaxation on Desorption Rate. J. Chem. Phys., 2013. 138: p. 154303.

35. Slanger, T.G. and G. Black, $\mathrm{CO}_{2}$ photolysis revisited. J. Chem. Phys., 1978. 68: p. 1844.

36. Bahr, D.A. and R.A. Baragiola, Photodesorption of solid $\mathrm{CO}_{2}$ by Lya. Astrophys. J., 2012. 761: p. 36.

37. Kim, K., The integrated intensity of the carbon monoxide fundamental band. J. Quant. Spectrosc. Radiat. Transfer, 1983. 30: p. 413. 


\section{Chapter 4: Lyman-a Photodesorption from $\mathrm{CO}_{2}$ (ice) at $75 \mathrm{~K}$ : Role of $\mathrm{CO}_{2}$ Vibrational Relaxation on Desorption Rate}

Modified from manuscript published on J. Chem. Phys. 138, 154303 (2013)

\subsection{Introduction}

The desorption of molecules from ices in interstellar space produces a part of the gas molecules observed by microwave spectroscopy [1, 2]. More than 180 molecules have so far been observed in interstellar space [3].Two processes which drive desorption from ices are thermal desorption[4] and radiation-driven desorption (photodesorption) [1]. Lyman-a radiation (10.2 eV) is a major component of radiation in space, originating widely from the recombination of electrons with atomic- $\mathrm{H}$ [5]. In a previous paper [6], we have shown that a $50 \mathrm{~nm}$ layer of $\mathrm{CO}_{2}$ (ice, $75 \mathrm{~K}$ ), upon photoexcitation by Lyman-a radiation, desorbs with an efficiency of about $\sim 0.008 \mathrm{CO}_{2}$ molecules/photon with a cross section of $\sim 7.3 \times 10^{-20} \mathrm{~cm}^{2}$ when desorption occurs from an ice containing a $50 \%-50 \%$ mixture of ${ }^{12} \mathrm{CO}_{2}$ and ${ }^{13} \mathrm{CO}_{2}$. The cross section for photo reaction of $\mathrm{CO}_{2}$ to

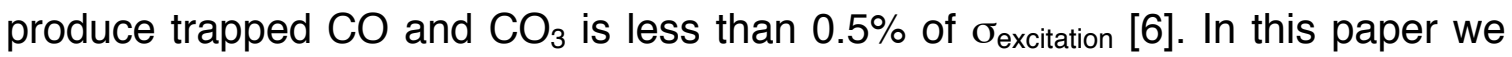
demonstrate how within the $\mathrm{CO}_{2}$ ice matrix, the matrix-mediated conversion of electronically-excited $\mathrm{CO}_{2}$ back to highly vibrationally-excited $\mathrm{CO}_{2}$ subsequently results in two main kinetic channels: (1). The vibrational relaxation of the excited molecule as it is retained in the ice matrix, and (2). The escape of the vibrationally-excited $\mathrm{CO}_{2}$ molecule by desorption from the ice. By varying the vibrational coupling between the excited molecule and the ice matrix, we find that the efficiency of $\mathrm{CO}_{2}$ photodesorption is strongly correlated inversely to the efficiency of $\mathrm{CO}_{2}$ vibrational relaxation in the ice. 


\subsection{Experimental}

The apparatus used in this work has been described in detail previously [6-8]. Briefly, a stainless steel high-vacuum IR cell ( $\mathrm{KBr}$ windows) equipped to study ice photo-desorption and photo-reaction by transmission IR spectroscopy, is used. The $\mathrm{CO}_{2}$ ice (nominal thickness $=50 \mathrm{~nm}$ ) is condensed at $75 \mathrm{~K}$ on a W grid whose openings are filled with compressed $\mathrm{KBr}$ powder. Exposure of the ice film to Lyman-a irradiation $\left(2.7 \times 10^{14}\right.$ photons $\left.\mathrm{cm}^{-2} \cdot \mathrm{s}^{-1}\right)$ is used to cause both photoreactions and photodesorption[6]. For the observation of small IR bands, the resolution of spectrometer may be decreased to $8 \mathrm{~cm}^{-1}$ to improve the signalto-noise ratio. The $\mathrm{CO}_{2}$ ice infrared absorption spectrum is characterized by the presence of inhomogeneously broadened (longitudinal) LO and (transverse) TO lattice modes $\left(v_{3}\right)$ [9]. The lineshape changes slightly as irradiation occurs, probably due to the disruption of the ice structure by irradiation [1]. Therefore we use the integrated absorbance of the $\left(v_{1}+v_{3}\right)$ combination mode, whose lineshape dose not change during irradiation, to follow the photodesorption of the $\mathrm{CO}_{2}$ ice. Pure ${ }^{12} \mathrm{CO}_{2}$ and ${ }^{13} \mathrm{CO}_{2}$ layers are separately studied and compared to mixed ices. A $50 \%-50 \%{ }^{12} \mathrm{CO}_{2}{ }^{-13} \mathrm{CO}_{2}$ isotopomer mixture and a $75 \%-25 \%{ }^{12} \mathrm{CO}_{2}$ ${ }^{13} \mathrm{CO}_{2}$ mixture are investigated to produce partial detuning of the vibrational spectrum of the $\mathrm{CO}_{2}$ isotopomers and their ice matrix. More extensive detuning due to oscillator dilution is achieved by using 1:1:8 $\mathrm{CO}_{2}$-Xe mixed ice with equimolar ${ }^{12} \mathrm{CO}_{2}$ and ${ }^{13} \mathrm{CO}_{2}$. Comparative studies of the $\mathrm{CO}_{2}$ isotopomer mixtures alone, or in Xe, provides an excellent basis for measurement of the effect of isotopic mass and of dilution effects on the photoprocesses.

\subsection{Results}

\subsubsection{Photodesorption from $50 \%-50 \%{ }^{12} \mathrm{CO}_{2}{ }^{13} \mathrm{CO}_{2}$ Mixed Ice}

Figure 4.1 shows the absorption spectrum of pure ${ }^{12} \mathrm{CO}_{2}$ and ${ }^{13} \mathrm{CO}_{2}$ ices of 50 $\mathrm{nm}$ thickness condensed on a $\mathrm{KBr}$ support at $75 \mathrm{~K}$. In addition, a nominal 50\%- 
$50 \%$ mixture of ${ }^{12} \mathrm{CO}_{2}$ and ${ }^{13} \mathrm{CO}_{2}$ is shown and also a $10 \%{ }^{12} \mathrm{CO}_{2}-10 \%{ }^{13} \mathrm{CO}_{2}$ mixture in $80 \% \mathrm{Xe}$ is shown. The absorbance scales are adjusted so that one can see the different relative rates of depletion of the $\mathrm{CO}_{2}$ isotopomers for each sample. Figure 4.1 shows the results of increasing the radiation dose on the four ices where photodepletion is the main process being observed. Only about $0.5 \%$ of the photo-induced processes involve the production of $\mathrm{CO}$ and $\mathrm{CO}_{3}$ species [6]. In Figure 4.1, it may be easily seen qualitatively by examining the $\mathrm{CO}_{2}$ peak intensity for each of the normalized absorption spectra that, during irradiation, making the matrix isotopically less pure causes a large increase in the relative rate of photodesorption for a constant fluence of $10.2 \mathrm{eV}$ radiation. Matrices of lower isotopic purity show a greater propensity for photodepletion. Xe dilution further enhances the relative rate of photodepletion of $\mathrm{CO}_{2}$.

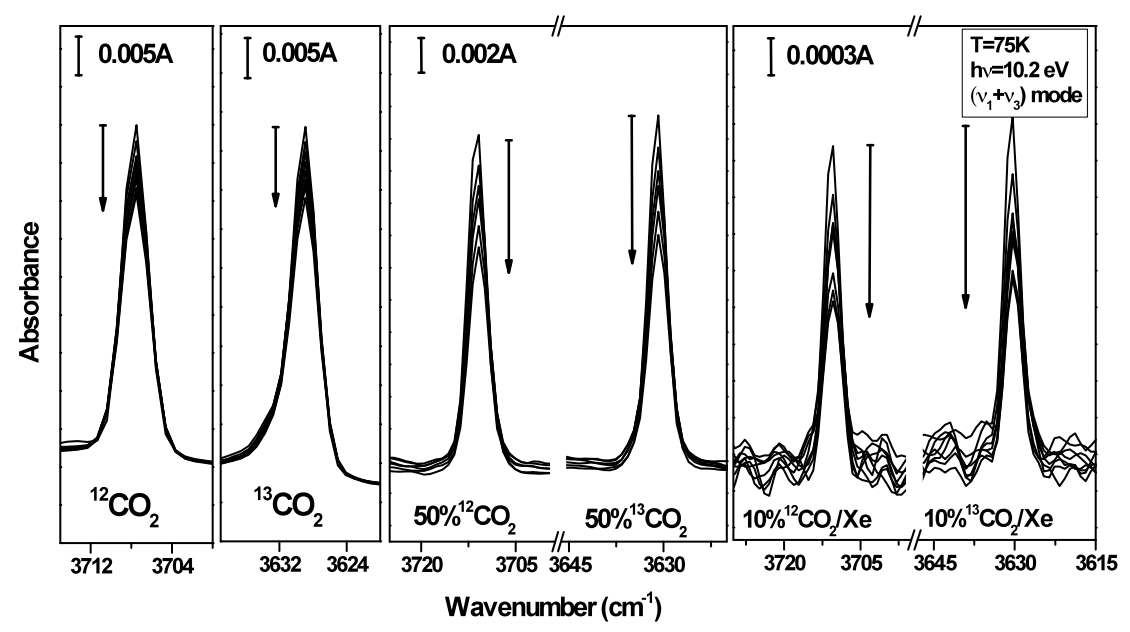

Figure 4.1. Photodepletion of $\mathrm{CO}_{2}$ from various ices by Lyman-a radiation at $75 \mathrm{~K}$. The Lyman- $\alpha$ fluence at the end of each experiment is $4.2 \times 10^{18}$ photons $\cdot \mathrm{cm}^{-2}$.

Figure 4.2 shows the comparison of the rates of photodepletion for the various pure and mixed ices studied. The curves are normalized at the point where the Lyman-a radiation is first admitted to the $\mathrm{CO}_{2}$ ices by opening an internal shutter. A correction for isothermal desorption at $75 \mathrm{~K}$ is made based on experiments made before the shutter is opened. Initially, before exposure to the UV radiation, 
the absorbance decreases slightly $\left(m_{1}\right)$ due to isothermal desorption at $75 \mathrm{~K}$. While thermal desorption is slow for the two $\mathrm{CO}_{2}$ matrices, for the $\mathrm{CO}_{2} / \mathrm{Xe}$ matrix, the thermal desorption $\left(m_{1}\right)$ is faster, indicating that the desorption activation energy for $\mathrm{CO}_{2}$ is diminished when $\mathrm{CO}_{2}$ neighbors are replaced by Xe neighbors in the ice. For all ices, the rate of photodesorption is measured by subtraction as $\left(m_{2}-m_{1}\right)$. In agreement with the behavior shown in Figure 4.1, one sees in Figure 4.2 that the photodesorption $\left(m_{2}-m_{1}\right)$ becomes more efficient as the isotopic dilution increases from isotopically pure $\mathrm{CO}_{2}$, to $50 \%-50 \%$ isotopic purity, to isotopic $\mathrm{CO}_{2}$ dilution in an $80 \%$ Xe matrix. The results of each measurement are summarized in Table 4.1, where the efficiency of $\mathrm{CO}_{2}$ desorption/photon is tabulated for the four types of matrix. For both $\mathrm{CO}_{2}$ isotopomers the efficiencies of desorption in the $50 \%-50 \%$ isotopic mixed ices are closely comparable for a given matrix.

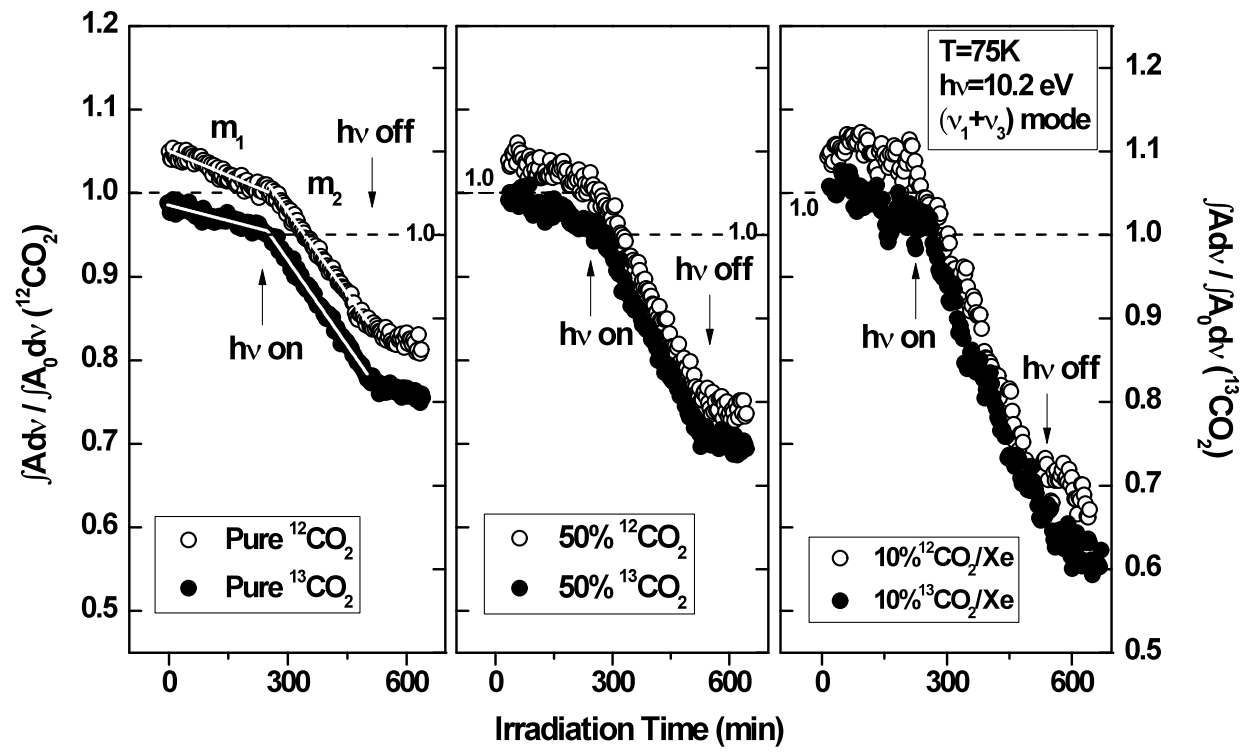

Figure 4.2. UV-induced Photodesorption of ${ }^{12} \mathrm{CO}_{2}$ and ${ }^{13} \mathrm{CO}_{2}$ in different ice matrices at $75 \mathrm{~K}$ 
Table 4.1. Comparison of the Efficiency of $\mathrm{CO}_{2}$ Photodesorption from Different Ice Matrices at $\mathrm{T}=75 \mathrm{~K}$

\begin{tabular}{|c||c|c||c|c||c|c||}
\hline $\begin{array}{c}\text { Rates of } \\
\text { Desorption }\end{array}$ & ${ }^{12} \mathrm{CO}_{2}$ (Pure) & ${ }^{13} \mathrm{CO}_{2}($ Pure $)$ & ${ }^{12} \mathrm{CO}_{2}(\mathbf{5 0} \%)$ & ${ }^{13} \mathrm{CO}_{2}(\mathbf{5 0} \%)$ & ${ }^{12} \mathrm{CO}_{2} / \mathrm{Xe}$ & ${ }^{13} \mathrm{CO}_{2} / \mathrm{Xe}$ \\
\hline $\begin{array}{c}\mathrm{m}_{1} \\
\left(\times 10^{-6}\right. \\
\text { fraction/s })\end{array}$ & $-(2.6 \pm 0.3)$ & $-(2.1 \pm 0.3)$ & $-(2.6 \pm 0.5)$ & $-(3.1 \pm 0.4)$ & $-(3.6 \pm 0.9)$ & $-(4.4 \pm 1.0)$ \\
\hline $\begin{array}{c}\mathrm{m}_{2} \\
\left(\times 10^{-6}\right. \\
\text { fraction/s })\end{array}$ & $-(10.9 \pm 0.1)$ & $-(11.3 \pm 0.1)$ & $-(15.6 \pm 0.2)$ & $-(15.4 \pm 0.2)$ & $-(22.8 \pm 0.4)$ & $-(20.8 \pm 0.4)$ \\
\hline $\begin{array}{c}\mathrm{m}_{2}-\mathrm{m}_{1} \\
\left(\times 10^{-6}\right. \\
\text { fraction/s })\end{array}$ & $-(8.3 \pm 0.4)$ & $-(9.2 \pm 0.4)$ & $-(13.0 \pm 0.7)$ & $-(12.3 \pm 0.6)$ & $-(19.2 \pm 1.3)$ & $-(16.4 \pm 1.4)$ \\
\hline $\begin{array}{c}\text { Efficiency } \\
\left(\mathrm{CO}_{2} / \text { photon }\right)\end{array}$ & $(5.6 \pm 0.3) \times 10^{-3}$ & $(6.2 \pm 0.3) \times 10^{-3}$ & $(8.8 \pm 0.5) \times 10^{-3}$ & $(8.4 \pm 0.4) \times 10^{-3}$ & $10^{-3}$ & $10^{-3}$ \\
\hline
\end{tabular}

\subsubsection{Photodesorption from $75 \%-25 \%{ }^{12} \mathrm{CO}_{2}{ }^{-13} \mathrm{CO}_{2}$ Mixed Ice}

The experiments described previously have employed either pure ${ }^{12} \mathrm{CO}_{2}$ or ${ }^{13} \mathrm{CO}_{2}$ ices or the symmetrical $50 \%-50 \%{ }^{12} \mathrm{CO}_{2}-{ }^{13} \mathrm{CO}_{2}$ ices. In the pure ice, a given vibrationally-excited isotopic $\mathrm{CO}_{2}$ molecule sees an identical isotopomer in all of its interactions with the matrix. Hence both pure ices should desorb with the same efficiency as shown in Table 4.1 . For $50 \%-50 \%$ isotopic ices, both $\mathrm{CO}_{2}$ isotopomers experience an environment involving $1 / 2 \mathrm{CO}_{2}$ molecules isotopically like themselves and $1 / 2 \mathrm{CO}_{2}$ molecules which are vibrationally dissimilar. Hence in the $50 \%-50 \%$ mixture the ${ }^{12} \mathrm{CO}_{2}$ should desorb with the same efficiency as the ${ }^{13} \mathrm{CO}_{2}$. But the desorption efficiencies for both ${ }^{12} \mathrm{CO}_{2}$ and ${ }^{13} \mathrm{CO}_{2}$ in the $50 \%-50 \%$ mixed ice is higher than in isotopically pure $\mathrm{CO}_{2}$. This is confirmed in Table 4.1.

We have performed a photodesorption study on a $75 \%-25 \%{ }^{12} \mathrm{CO}_{2}-{ }^{13} \mathrm{CO}_{2}$ mixed ice. Here, in contrast to the studies previously described, a vibrationally- 
excited ${ }^{12} \mathrm{CO}_{2}$ molecule is statistically more strongly coupled to ${ }^{12} \mathrm{CO}_{2}$ molecules in the matrix and a ${ }^{13} \mathrm{CO}_{2}$ molecule is statistically more weakly coupled to its ${ }^{13} \mathrm{CO}_{2}$ neighbors in the matrix. Hence, if vibrational interactions control the rate of energy exchange, the ${ }^{12} \mathrm{CO}_{2}$ molecule will be more rapidly relaxed by the lattice, and its desorption rate will therefore be diminished compared to the ${ }^{13} \mathrm{CO}_{2}$ molecule which cannot relax so efficiently. This expectation is in accord with experiment as shown in Figure 4.3. Here, the majority ${ }^{12} \mathrm{CO}_{2}$ isotopomer desorbs more slowly than the minority ${ }^{13} \mathrm{CO}_{2}$ isotopomer since ${ }^{12} \mathrm{CO}_{2}$ is more strongly relaxed by vibrational coupling to the matrix. The errors in this experiment is near the limit of sensitivity of our measurements, where the resolution of the spectrometer has been reduced to $8 \mathrm{~cm}^{-1}$ to gain more signal-to-noise ratio.

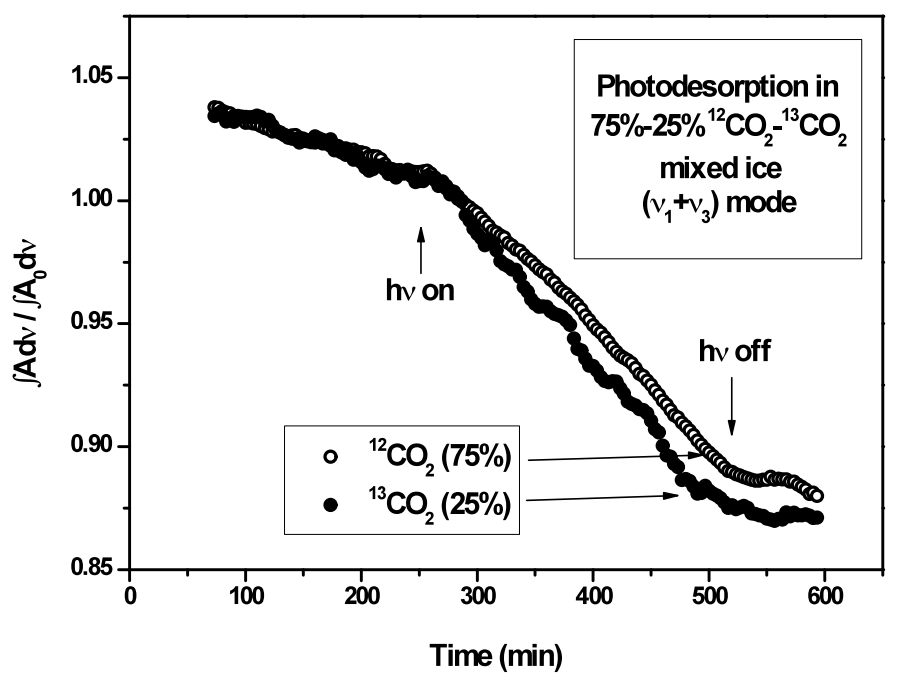

Figure 4.3. UV-Induced photodesorption of ${ }^{12} \mathrm{CO}_{2}$ and ${ }^{13} \mathrm{CO}_{2}$ in an unsymmetrical ${ }^{12} \mathrm{CO}_{2}(75 \%)$ and ${ }^{13} \mathrm{CO}_{2}(25 \%)$ mixed ice at $75 \mathrm{~K}$. Each of the ${ }^{13} \mathrm{CO}_{2}$ data points is averaged from 5 original spectra to reduce the noise. 


\subsection{Discussion}

\subsubsection{Photochemical Processes in $\mathrm{CO}_{2}$ (ice)-General}

Figure 4.4 illustrates the overall combination of reaction steps observed in this, and previous work $[1,6]$, when $\mathrm{CO}_{2}$ ice is irradiated by Lyman-a radiation at $75 \mathrm{~K}$. An initial electronic excitation process occurs to produce $\mathrm{CO}_{2}{ }^{*}$ species. These species are characterized by the lengthening of a $\mathrm{C}-\mathrm{O}$ bond on the upper repulsive potential curves corresponding to the behavior of electronically excited $\mathrm{CO}_{2}{ }^{*}$ in the ice. As shown previously, a distinct kinetic isotope effect (KIE) is associated with this dissociation step [6], where the isotopic product, ${ }^{12} \mathrm{CO}$, is favored by about $10 \%$ over ${ }^{13} \mathrm{CO}$ in the dissociation process. On the right hand side of Figure 4.4, a pair of reactions, involving $\mathrm{CO}$ and $\mathrm{O}$ trapped in the $\mathrm{CO}_{2}$ matrix, produce $\mathrm{CO}_{3}$, as well as traces of $\mathrm{O}_{3}$ (not shown). The $\mathrm{CO}, \mathrm{CO}_{3}$ and $\mathrm{O}_{3}$ species are produced and trapped in the ice, and are detected by IR spectroscopy [6]. The primary KIE is governed by deexcitation probability effects where $\mathrm{CO}_{2}{ }^{*}$ exchanges electronic energy with the ice matrix, as $\Delta \mathrm{E}^{*}$, during the CO-O bond stretching process [6]. This quenching reaction sensitively controls the isotopic ratio of ${ }^{12} \mathrm{CO}$ and ${ }^{13} \mathrm{CO}$ produced in the excitation step on the upper $\mathrm{CO}_{2}{ }^{*}$ potential energy [6]. 


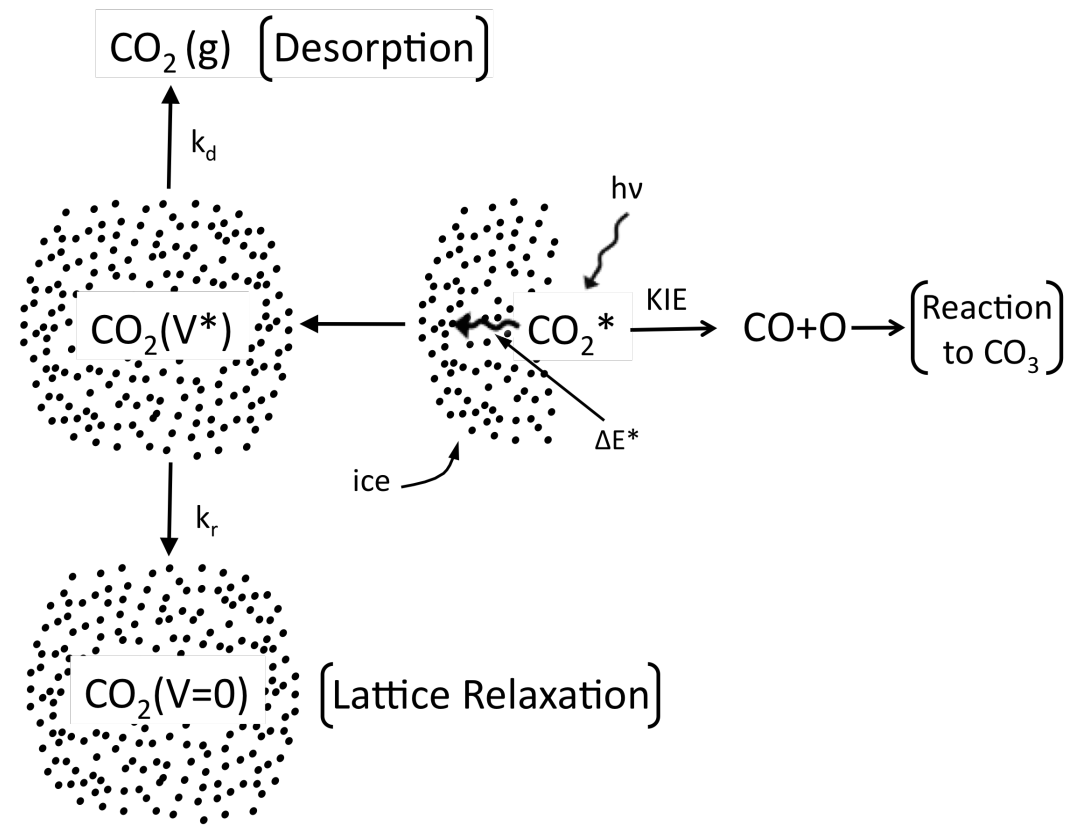

Figure 4.4. Kinetic pathways of confined $\mathrm{CO}_{2}$ in $\mathrm{CO}_{2}$ (ice)

After $\mathrm{CO}_{2}{ }^{*}$ is electronically quenched in its $\mathrm{CO}_{2}$ cage, a highly vibrationallyexcited $\mathrm{CO}_{2}\left(\mathrm{~V}^{*}\right)$ molecule is produced in the ice matrix. It can choose to desorb via process, $k_{d}$, or to vibrationally quench by the relaxation process, $k_{r}$. As is experimentally shown in this investigation, the vibrational relaxation process is strongly sensitive to the composition of the matrix surrounding $\mathrm{CO}_{2}\left(\mathrm{~V}^{*}\right)$ and to the ability of the matrix to efficiently absorb energy from $\mathrm{CO}_{2}\left(\mathrm{~V}^{*}\right)$. The magnitude of the vibrational relaxation process in the lattice subsequently governs the rate of the desorption process, $\mathrm{k}_{\mathrm{d}}$, with faster vibrational relaxation, $\mathrm{k}_{\mathrm{r}}$, being associated with slower desorption. Although we present $k_{r}$ and $k_{d}$ as rate constants, this is highly schematic, as the postulate of thermal equilibrium is inappropriate for the highly excited $\mathrm{CO}_{2}\left(\mathrm{~V}^{*}\right)$ species as it returns in its matrix from the upper electronically-excited $\mathrm{CO}_{2}{ }^{*}$ state. 


\subsubsection{Vibrational Tuning of the Ice Matrix to Control $\mathrm{CO}_{2}\left(\mathrm{~V}^{*}\right)$ Relaxation and Desorption}

Figure 4.5 shows a schematic potential energy diagram where electronicallyexcited $\mathrm{CO}_{2}{ }^{*}$ species are produced and evolve. The production of $\mathrm{CO}_{2}\left(\mathrm{~V}^{*}\right)$ by deexcitation from $\mathrm{CO}_{2}{ }^{*}$ occurs at high levels of vibrational excitation. The matrix surrounding $\mathrm{CO}_{2}\left(\mathrm{~V}^{*}\right)$ possesses identical vibrational states to the $\mathrm{CO}_{2}\left(\mathrm{~V}^{*}\right)$ species when the isotopic composition of $\mathrm{CO}_{2}\left(\mathrm{~V}^{*}\right)$ matches the matrix in the two experiments with isotopically pure $\mathrm{CO}_{2}$. This is postulated to lead to good vibrational coupling of $\mathrm{CO}_{2}\left(\mathrm{~V}^{*}\right)$, leading to good energy transfer to the $\mathrm{CO}_{2}$ lattice. As shown in Table 4.1, efficient coupling for the pure isotopomers leads to the lowest efficiencies of $\mathrm{CO}_{2}$ desorption (see ${ }^{12} \mathrm{CO}_{2}$ (pure) and ${ }^{13} \mathrm{CO}_{2}$ (pure) efficiencies of 5.8 and $6.2 \times 10^{-3} \mathrm{CO}_{2} /$ photon, respectively).

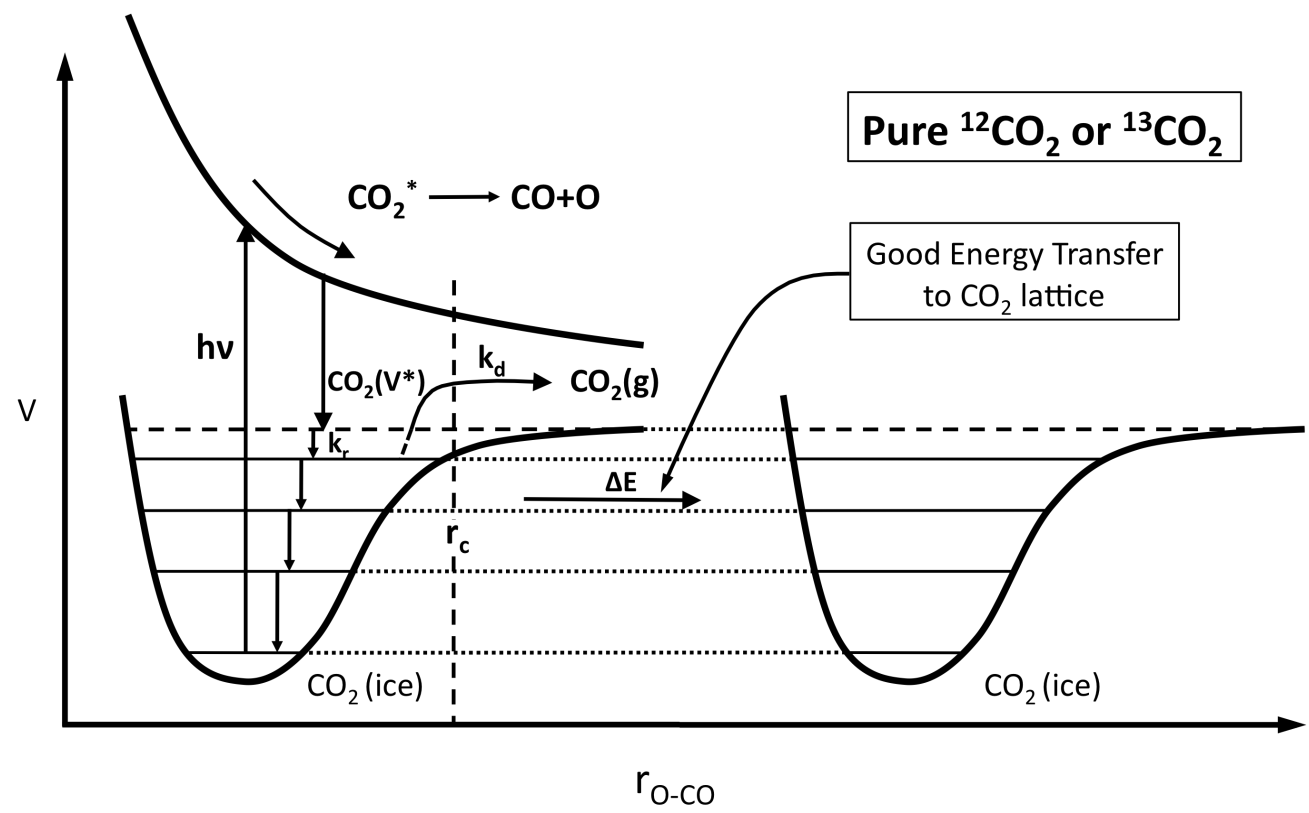

Figure 4.5. Role of vibrational relaxation in lattice ice in depleting $\mathrm{CO}_{2}\left(\mathrm{~V}^{*}\right)-$ Good coupling between pure $\mathrm{CO}_{2}$ molecules. When $\mathrm{O} \cdots \mathrm{CO}$ separation beyond a critical distance, $r_{c}$, occurs, photoreaction takes place.

Figure 4.6 shows a similar situation to that in Figure 4.5, except the lattice is vibrationally miss-matched to the $\mathrm{CO}_{2}\left(\mathrm{~V}^{*}\right)$ species, where either ${ }^{12} \mathrm{CO}_{2}\left(\mathrm{~V}^{*}\right)$ 
species is shown as partially decoupled to ${ }^{13} \mathrm{CO}_{2}$ matrix species, or vice versa. This poor vibrational coupling occurs in the 50\%-50\% mixed $\mathrm{CO}_{2}$ ice. Here photodesorption occurs with a higher efficiency as the lattice relaxation process is disfavored. As shown in Table $4.1,{ }^{12} \mathrm{CO}_{2}$ and ${ }^{13} \mathrm{CO}_{2}$ exhibit photodesorption efficiencies of 8.8 and $8.4 \times 10^{-3} \mathrm{CO}_{2} /$ photon respectively in the $50 \%-50 \%{ }^{12} \mathrm{CO}_{2}$ and ${ }^{13} \mathrm{CO}_{2}$ mixed ice, about $50 \%$ greater than that seen for the isotopically-pure $\mathrm{CO}_{2}$.

Further experiments were carried out with a $50 \%-50 \%$ isotopic mixture of $\mathrm{CO}_{2}$ isotopomers diluted with $80 \%$ xenon. This experiment tests the role of the average distance between $\mathrm{CO}_{2}$ oscillators separated by Xe spacer atoms. Here we see how the vibrational relaxation of the $50 \%-50 \% \mathrm{CO}_{2}$ ice is further diminished by increasing the $\mathrm{CO}_{2}-\mathrm{CO}_{2}$ intermolecular distance, leading to enhanced photo-desorption. As shown in Table 4.1, for both $\mathrm{CO}_{2}$ isotopomers in the Xe matrix, the efficiency of photodesorption is about $12 \times 10^{-3} \mathrm{CO}_{2} /$ photon, about 1.5 times that observed for the undiluted $50 \%-50 \%$ isotopic mixture.

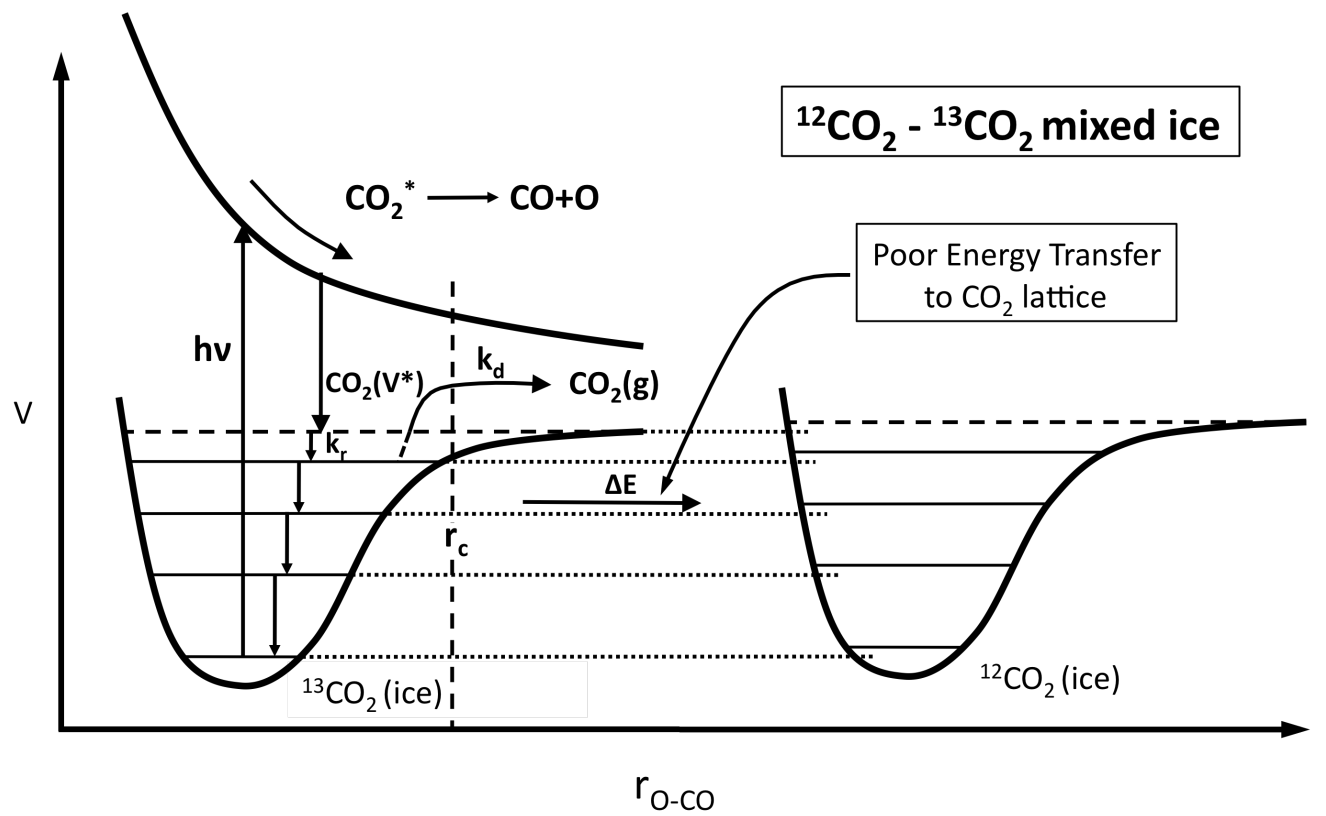

Figure 4.6. Role of vibrational relaxation in lattice ice in depleting $\mathrm{CO}_{2}\left(\mathrm{~V}^{*}\right)-$ Poor coupling between ${ }^{12} \mathrm{CO}_{2}$ and ${ }^{13} \mathrm{CO}_{2}$ molecules. 
Figure 4.3 shows another way of witnessing the importance of vibrational coupling in the relaxation of vibrationally-excited $\mathrm{CO}_{2}$ molecules in the matrix. By working with an unsymmetrical mixture of ${ }^{12} \mathrm{CO}_{2}(75 \%)$ and ${ }^{13} \mathrm{CO}_{2}(25 \%)$ we reasoned that the majority species would vibrationally relax in the matrix more rapidly than the minority, causing the majority species to desorb less rapidly than the minority species. This is observable in Figure 4.3, where the relative rates of majority and minority species photodesorption are in the ratio 0.8:1.0.

The experiments taken together show that vibrational mismatching (detuning) and increased $\mathrm{CO}_{2}-\mathrm{CO}_{2}$ intermolecular distances both enhance $\mathrm{CO}_{2}$ photodesorption efficiency as poor energy transfer conditions are built into the matrix surrounding a $\mathrm{CO}_{2}\left(\mathrm{~V}^{*}\right)$ molecule, controlling whether $\mathrm{CO}_{2}$ desorbs or relaxes vibrationally in the matrix.

\subsubsection{Details of Vibrational Relaxation and Photodesorption in $\mathrm{CO}_{2}$ Ice}

The predominant Lyman-a driven photoprocess in $\mathrm{CO}_{2}$ ice involves the return of the electronically excited molecule to the ground state as highly vibrationallyexcited $\mathrm{CO}_{2}$. The production of a vibrationally-excited $\mathrm{CO}_{2}\left(\mathrm{~V}^{*}\right)$ species in the $\mathrm{CO}_{2}$ (ice) results either in $\mathrm{CO}_{2}$ desorption or in the vibrational relaxation and capture of $\mathrm{CO}_{2}\left(\mathrm{~V}^{*}\right)$ as it interacts with the matrix. Our studies of the dependence of the desorption rate on the composition of the matrix indicates that coupling of $\mathrm{CO}_{2}$ vibrational states in the matrix is strongly correlated (inversely) with the $\mathrm{CO}_{2}$ desorption rate. Strong vibrational relaxation leads to slower desorption.

The coupling of a vibrationally-excited molecule to a matrix in which it is embedded has been widely studied for example in Refs. [10-13]. It is believed that two long range dipole-dipole processes are mainly responsible for energy transfer from the molecule to the matrix. If there is vibrational frequency matching between the excited molecule and those of the matrix, then a resonance-type of energy transfer occurs without any change in vibrational level for either molecule. 
This is termed energy migration and such energy migration strongly enhances the range of the interaction and vibrational energy transfer throughout the matrix [10]. Energy migration is diminished even if two isotopically-matched molecules are affected by the presence of a third nearby isotopically mismatched molecule [10]. The energy migration phenomenon is similar to a Förster process for electronic energy levels. It should have an $r^{-6}$ distance dependence [10]. If vibrational energy matching is not present, then energy transfer can occur by less efficient steps in the ice via phonon-assisted processes where the phonons either add or subtract energy to facilitate the exchange of vibrational energy. The role of phonons is well illustrated by the fact that mismatching of vibrational energy in ice by isotopic labeling can result in the IR fluorescence of the mismatched molecule when its mismatched isotopic counterpart is excited directly [10]. Energy relaxation from highly-excited polyatomic molecules may occur by cascade processes within the molecule as well as for the polyatomic molecules in the matrix to which energy has been transferred [14]. Energy transfer for polyatomic molecules is expected to be rapid compared to that observed for diatomic molecules [13] due to the higher density of vibrational states. Energy can flow from an excited molecule to the matrix when the vibrational energy is much higher than $\mathrm{k}_{\mathrm{B}} \mathrm{T}$ as is true for $\mathrm{CO}_{2}$ in these studies. For lower vibrational energies where the vibrational energy is $\sim k_{B} T$ or lower, energy can flow both ways [14].

Figures 5 and 6 schematically illustrate vibrationally-matched and mismatched molecules in an ice matrix. For vibrationally-matched $\mathrm{CO}_{2}$ molecules, as in our experiments on almost isotopically pure ${ }^{12} \mathrm{CO}_{2}$ and ${ }^{13} \mathrm{CO}_{2}$, as shown in the two left panels of Figure 4.1, optimized coupling is expected for two reasons: (1). The frequencies of the neighbor $\mathrm{CO}_{2}$ oscillators are matched; (2). The intermolecular distance between coupled oscillators is lowest. The photodesorption efficiency for both ${ }^{12} \mathrm{CO}_{2}$ (pure) and ${ }^{13} \mathrm{CO}_{2}$ (pure) is lowest among these experiments, namely about $6 \times 10^{-3} \mathrm{CO}_{2}$ /photon as seen in Table 4.1. The middle panel in Figure 4.1 corresponds to the $50 \%-50 \%$ mixture of ${ }^{12} \mathrm{CO}_{2}$ and ${ }^{13} \mathrm{CO}_{2}$. Here, vibrational 
coupling is reduced from that for the isotopically-pure ices; as well the average distance between isotopomers with the same mass is slightly increased. The photodesorption efficiency is increased to $8.6 \times 10^{-3} \mathrm{CO}_{2} /$ photon for both isotopomers as seen in Table 4.1.

Additionally, when the $50 \%-50 \%$ isotopic mixture is diluted by 1:1:8 using Xe as a diluent, the photodesorption efficiency is increased to its highest values in these experiments, about $12 \times 10^{-3} \mathrm{CO}_{2}$ /photon as seen in Table 4.1. The $\mathrm{Xe}$ experiments involving a $50 \%-50 \%$ isotopic mixture of $\mathrm{CO}_{2}$ molecules, compared to the undiluted $50 \%-50 \%$ isotopic mixture, show that a distance effect operates also in this system. In the case of the Xe mixture with $\mathrm{CO}_{2}$, the $\mathrm{CO}_{2}-\mathrm{CO}_{2}$ intermolecular distance is about 1.89 of that in the $50 \%-50 \%$-isotopic $\mathrm{CO}_{2}$ mixture.

And finally, as shown in Figure 4.3, when the matrix is made isotopically unsymmetrical, the dominant isotopomer exhibits the lower photodesorption rate compared to the minority isotopomer because the dominant isotopomer is more readily relaxed by more efficient vibrational coupling in the mixed ice.

In the studies in reference [6], the cross sections for desorption and relaxation from the $50 \%-50 \%$ CO isotopic mixture were measured as: $\sigma_{\text {relaxation }}=8.9 \times 10^{-18}$ $\mathrm{cm}^{2} ; \sigma_{\text {desorption }}=3.3 \times 10^{-20} \mathrm{~cm}^{2}$. For the range of matrices studied here (Table 4.1), the efficiency of $\mathrm{CO}_{2}$ photodesorption varies over a factor of about 2 implying that small fractional variation of relaxation efficiency will greatly change the efficiency of photodesorption.

\subsubsection{Efficiency of Vibrational Relaxation in $\mathrm{CO}_{2}$ (ice)}

Theoretical investigations have shown that when an oscillator molecule is embedded in a monoatomic ice matrix, the efficiency of the exchange of vibrational energy with the phonon bath is poor $[15,16]$. Measurements made at $5-10 \mathrm{~K}$ yield long vibrational lifetimes in the $10^{-3} \mathrm{~s}$ range, as experimentally demonstrated for $\mathrm{CO}$ in an Ar matrix in the temperature range of $6-18 \mathrm{~K}$ (see 
Table 4.1 and III in Ref. 15). For $\mathrm{O}_{2}$ in an Ar matrix, vibrational relaxation times of the order of 100's of seconds are found for $v=4$ [13]. The great mismatch between the $\mathrm{O}_{2}$ vibrational frequency and the frequency distribution in the lattice phonons causes the slow relaxation. As the temperature is raised the vibrational lifetime will decrease by several orders of magnitude, all other things being equal [15].

For our experiments where neighbor $\mathrm{CO}_{2}$ oscillators are present in all cases, vastly smaller vibrational lifetimes are involved at $75 \mathrm{~K}$. Thus, if Lyman-a photons are incident at a flux of $2.7 \times 10^{14}$ photons $\cdot \mathrm{cm}^{-2} \cdot \mathrm{s}^{-1}$, and if 0.63 of this radiation is absorbed in the $50 \mathrm{~nm} \mathrm{CO}_{2}$ (ice) film [6] and then essentially converted either to desorption or lattice relaxation, the time constant for relaxation is $\tau \approx 10^{-12} \mathrm{~s}$ [17], about 6 orders of magnitude faster relaxation than expected in a monoatomic matrix at $75 \mathrm{~K}$. This shows the importance for vibrational energy transfer to the matrix of having high frequency vibrational modes in the matrix where the energy gap between the excited oscillator and its neighbors is small compared to that for phonon modes in a monoatomic atom matrix.

Our experiments show that the desorption rate is inversely correlated to the vibrational relaxation rate. For the $\mathrm{CO}_{2}$ lattices and for the $\mathrm{CO}_{2}$-Xe lattice investigated here, we are in a regime of relatively high vibrational relaxation efficiency. Should lattice composition conditions move into the regime of slower vibrational relaxation, an increase in photodesorption rate would occur. For astrochemical conditions, where impure ices generally exist containing molecules with many vibrational modes, the effect of different ice compositions would be expected to exhibit only a small effect on the photodesorption rate due to the broad spectrum of molecular oscillator frequencies in the mixed ice.

We have shown that in an isotopically unsymmetrical ice, such as $75 \%{ }^{12} \mathrm{CO}_{2}$ : $25 \%{ }^{13} \mathrm{CO}_{2}$ the minority isotopomer will photodesorb with the highest rate because its coupling to the ice lattice in diminished compared to the majority isotopomer. This observation implies that all other things being equal, photodesorption of isotopically-labeled species from more chemically- 
homogeneous ices will be governed by the degree of vibrational matching in the lattice. In this case, photodesoption would favor the minority mismatched molecule, and its enhanced desorption would cause the degree of isotopic purity of the ice to increase.

\subsection{Summary of Results}

The following results have been obtained in this investigation:

1. The Lyman-a photodesorption of $\mathrm{CO}_{2}$ from a $\mathrm{CO}_{2}$ ice lattice is strongly mediated by the relaxation of vibrationally-hot $\mathrm{CO}_{2}$ molecules which evolve from the deexcitation of electronically-excited $\mathrm{CO}_{2}$ molecules.

2. The rate of vibrational relaxation depends on the composition of the matrix enclosing the vibrationally-excited $\mathrm{CO}_{2}$ molecule. Matrices containing $\mathrm{CO}_{2}$ molecules which are vibrationally in-tune with the excited $\mathrm{CO}_{2}$ molecule are most efficient in the relaxation process. As the matrix is detuned by isotopic dilution or by dilution in $\mathrm{Xe}$, vibrational relaxation decreases.

3. The efficiency of vibrational relaxation of $\mathrm{CO}_{2}$ mediates the photodesorption of $\mathrm{CO}_{2}$. Less efficient vibrational relaxation is associated with more efficient photodesorption, such that the cross sections for desorption and relaxation, summed together, are constant.

4. Vibrational relaxation in ices is thought to involve resonant interactions between oscillators of the same frequency as well as less efficient phononassisted relaxation which compensates for vibrational mismatch.

5. Highly excited $\mathrm{CO}_{2}\left(\mathrm{~V}^{*}\right)$ species in the $\mathrm{CO}_{2}$ ices studied here at $75 \mathrm{~K}$ have a vibrational lifetime of $\tau \approx 10^{-12} \mathrm{~s}$, many orders of magnitude below the lifetime expected for the excited $\mathrm{CO}_{2}$ molecule in a monoatomic inert gas matrix, where energy exchange is inhibited by the large energy gap with the phonon modes.

6. The results show that under astronomical conditions where ices are being photodesorbed in interstellar space and vibrational lifetimes in the impure ice matrices are short, the rate of photodesorption will depend on the ice composition 
and the ice's ability to drain excess vibrational energy from hot molecules produced by electronic quenching effects in the ice matrix. Effects such as matrix density and composition will mediate photodesorption processes in the interstellar medium, and for isotopomers in more pure ices, the minority isotopic species will desorb more rapidly.

\subsection{References:}

1. Oberg, K.I., E.F. van Dishoeck, and H. Linnartz, Photodesorption of ices I: CO, $\mathrm{N}_{2}$, and $\mathrm{CO}_{2}$. Astron. Astrophys., 2009. 496: p. 281.

2. Boogert, A.C.A. and P. Ehrenfreund, Interstellar Ices, in Astrophysics of Dust, ASP Conference Series, A.N. Witt, G.C. Clayton, and B.T. Draine, Editors. 2004. p. 547.

3. Woon, D.E., Interstellar and Circumstellar Molecules. http://www.astrochymist.org/astrochymist ism.html.

4. Viti, S., et al., Evaporation of ices near massive stars: models based on laboratory temperature programmed desorption data. Mon. Not. R. Astron. Soc. , 2004. 354: p. 1141.

5. Weinberg, D.H., et al., The Lyman-a Forest as a Cosmological Tool, in American Institute of Physics Conference Series, 2003. p. 157.

6. Yuan, C. and J.T. Yates, Jr., Isotope Effect in the Photochemical Decomposition of $\mathrm{CO}_{2}$ (ice) by Lyman-a Radiation. J. Chem. Phys., 2013. 138: p. 154302.

7. Rajappan, M., et al., Photochemical Decomposition of $\mathrm{N}_{2} \mathrm{O}$ by Lyman- $\mathrm{a}$ Radiation: Scientific Basis for a Chemical Actinometer. J. Phys. Chem. A, 2010. 114: p. 3443.

8. Rajappan, M., C. Yuan, and J.T. Yates, Jr., Lyman-a driven molecule formation on $\mathrm{SiO}_{2}$ surfaces - connection to astrochemistry on dust grains in the interstellar medium. J. Chem. Phys., 2011. 134: p. 064315.

9. Ovchinnikov, M.A. and C.A. Wight, Inhomogeneous broadening of infrared and Raman spectral bands of amorphous and polycrystalline thin films. J. Chem. Phys., 1993. 99: p. 3374. 
10. Dubost, $H$. and R. Charneau, Laser studies of vibrational energy transfer and relaxation of CO trapped in solid neon and argon. Chem. Phys., 1976. 12: p. 407.

11. Fong, F.K., ed. Topics in Applied Physics. Radiationless Processes in Molecules and Condensed Phases. Vol. 15. 1976, Springer: New York.

12. Englman, R., Non-Radiative Decay of lons and Molecules in Soilds1979, New York: North-Holland.

13. Nitzan, A., Chemical Dynamics in Condensed Phases2006: Oxford University Press.

14. Tokmakoff, A. Time-Dependent Quantum Mechanics: 10.1 Vibrational Relaxation (course note). 2009 [cited 2012 Oct.]; Available from: Massachusetts Institute of Technology: MIT OpenCouseWare, http://ocw.mit.edu. License: Creative Commons BY-NC-SA.

15. Diestler, D.J., Nonradiative vibrational relaxation of diatomic molecules isolated in solid rare-gas matrices. J. Chem. Phys., 1974. 60: p. 2692.

16. Nitzan, A., S. Mukamel, and J. Jortner, Some features of vibrational relaxation of a diatomic molecule in a dense medium. J. Chem. Phys., 1974. 60: p. 3928.

17. Baggen, M. and A. Lagendijk, Time resolved study of vibrational relaxation in solid CO2 at high pressures. Chemical Physics Letters, 1991. 177: p. 361. 


\section{Chapter 5: Radiation Damage and Associated Phase Change Effect on Photodesorption Rates from Ices - Lyman- $\alpha$ Studies of the Surface Behavior of $\mathrm{CO}_{2}$ (ice)}

Modified from manuscript published on The Astrophysical Journal. 780, 8 (2014)

\subsection{Introduction}

Photodesorption from ices is thought to be a source of gas phase molecules in the interstellar medium (ISM) [1-4]. The destructive interaction between extreme ultraviolet (EUV) radiation and ices has been shown to greatly enhance the rate of photodesorption; it is believed that irradiation with photons or electrons will cause photosensitive ices to become more porous and to exhibit weaker intermolecular bonding as crystal disorder is produced [5]. However in contrast, ices subject to ion bombardment or cosmic ray damage in space, become compacted due to momentum transfer effects from the incoming particle to the ice crystal $[6,7]$. The multiple origins of EUV radiation in the ISM have been discussed by Gerakines et al. (1996).

It has been observed in several studies of photodesorption from ices that an induction period occurs at the beginning of irradiation [8,9]. This delayed onset of the rapid evolution of photoproducts is thought to be due to photon-induced ice disordering related to the photodecomposition and desorption of a fraction of the molecules composing the ice. Radiation induced changes in the ice structure influences the photodesorption rate of molecules as well as the trapping of photoproducts.

In studies of Lyman- $\alpha$ induced $\mathrm{CO}_{2}$ photodesorption, Öberg et al. (2009b) observed a delayed photodesorption of $\mathrm{CO}$ from crystalline $\mathrm{CO}_{2}$ (ice) at $40-60 \mathrm{~K}$, 
but this delay did not occur for amorphous ice deposited at $18 \mathrm{~K}$. They also compared the photodesorption yield of thermally-annealed $\mathrm{CO}_{2}$ (ice) with unannealed ice at $18 \mathrm{~K}$, finding that the former yield is $\sim 0.4$ times that of the latter. This indicates that the $\mathrm{CO}$ photodesorption yield is dependent on the ice structure and that annealing of amorphous $\mathrm{CO}_{2}$ (ice) reduces the $\mathrm{CO}$ desorption rate. Similar observations were made by Bahr et al. [9]. In this report we show that large structural changes in the $\mathrm{CO}_{2}$ (ice) occur during Lyman-a induced photochemistry. In a $50 \mathrm{~nm} \mathrm{CO}$ ice film at $75 \mathrm{~K}$, radiation damage increases the $\mathrm{CO}$ photodesorption rate by $\sim 90$ fold and the $\mathrm{CO}_{2}$ photodesorption rate by $\sim 8$ fold. These effects are only observed readily using a type of photodesorption measurement particularly sensitive to the kinetics of desorption from the ice surface. We demonstrate that radiation damage, causing crystal structure disorder to ices, produces new more efficient molecular transport pathways which are accessed by photoexcitation processes.

Here we focus on the initial stages (first $8 \%$ ) of the Lyman-a photodepletion from $\mathrm{CO}_{2}$ (ice), directly measuring both $\mathrm{CO}_{2}(\mathrm{~g})$ and $\mathrm{CO}(\mathrm{g})$ evolution. We observe changes both inside the ice and outside the ice (gas phase) by combining transmission IR spectroscopy through the ice film with simultaneous gas phase analysis of desorbing gases using a quadrupole mass spectrometer (QMS) in a pumped vacuum system. It is observed that $\mathrm{CO}$ (and $\mathrm{CO}_{3}$ ) are formed and trapped inside the $\mathrm{CO}_{2}$ (ice) matrix immediately upon irradiation. Continued irradiation causes the ice structure to disorder extensively. Thus as radiationinduced ice damage occurs, more and more $\mathrm{CO}_{2}$ and trapped $\mathrm{CO}$ molecules are delivered per unit time at constant Lyman-a flux from the ice surface into the gas phase as additional facile transport pathways are provided for molecular escape from the ice. Such radiation damage effects will therefore have to be considered in models concerned with the gas phase composition of regions of the ISM where photodesorption from ices is a major factor in supplying gas phase products. This 
paper is concerned with the Lyman- $\alpha$ radiation damage process in pure $\mathrm{CO}_{2}$ ice, and serves as a baseline for understanding the behavior of more complex mixed ices likely to be found in the ISM.

\subsection{Experimental}

The experiment was carried out in a stainless steel high-vacuum cell with a base pressure of $1 \times 10^{-8}$ Torr, as described in detail previously [10-12]. Transmission IR spectroscopy is employed to measure the change of the $\mathrm{CO}_{2}$ (ice) absorption spectra and the formation of new species during irradiation. The Lyman-a irradiation $\left(10.2 \mathrm{eV} ;(2.7 \pm 0.7) \times 10^{14}\right.$ photons $\left.\mathrm{cm}^{-2} \cdot \mathrm{s}^{-1}\right)$ is produced by an rf-activated hydrogen discharge lamp and transmitted through a $\mathrm{MgF}_{2}$ window $[10,13]$. The Lyman-a exposure is controlled by a shutter in vacuum placed in front of the sample. In a typical measurement, a nominal $50 \mathrm{~nm} \mathrm{CO} \mathrm{CO}_{2}(\sim 135$ monolayers $(\mathrm{ML})$ assuming the layer density is $8 \times 10^{14}$ molecules $(\mathrm{ML})^{-1}$ based on the ice density at $75 \mathrm{~K}$ ) ice film is condensed at $75 \mathrm{~K}$ as a crystalline ice [14] onto a large number of neighboring $\mathrm{KBr}$ platlets, each of $0.022 \times 0.022 \mathrm{~cm}^{2}$ dimension, produced by compression of $\mathrm{KBr}$ powder into a $\mathrm{W}$ grid using 3000 psi. The compressed $\mathrm{KBr}$ disk of $0.7 \mathrm{~cm}$ diameter exposes $\sim 0.35 \mathrm{~cm}^{2}$ of the ice film on $\mathrm{KBr}$ to Lyman-a radiation arriving at a $45^{\circ}$ angle. This method of support for the ice gives very efficient thermal contact between the grid, the $\mathrm{KBr}$ support platlets and the ice film as well as excellent IR transmission. The $\mathrm{CO}_{2}$ film can be deposited with a thickness reproducibility of $\pm 10 \%$. No evidence for $\mathrm{CO}_{2}$ penetration into the $\mathrm{KBr}$ supporting material is seen by IR spectroscopy. No evidence for $\mathrm{H}_{2} \mathrm{O}$ or other impurity condensation on the $\mathrm{CO}_{2}$ film is seen by careful IR measurements. The characteristic (1/e) penetration depth of Lyman-a photons in $\mathrm{CO}_{2}$ (ice) is measured to be approximately $50 \mathrm{~nm}$ [11]; therefore about $2 / 3$ of the incident light irradiation is absorbed by the ice film. The freshly- 
prepared ice film at $75 \mathrm{~K}$ is kept in vacuum for 3-4 hours before the Lyman- $\alpha$ radiation is admitted in order to stabilize the film properties. Liquid $\mathrm{N}_{2}$, sometimes cooled below $77 \mathrm{~K}$ by He bubbling, is used as the refrigerant [15]. We find that the ice film temperature on the grid is $5 \mathrm{~K}$ higher than the reentrant Dewar temperature.

The cell is always open to a turbo molecular pump and an ion pump with a combined constant pumping speed, $S$ (for $\mathrm{CO}, \mathrm{S}_{\mathrm{CO}}=0.21 \mathrm{~L} \cdot \mathrm{S}^{-1}$; for $\mathrm{CO}_{2}$, $\left.\mathrm{S}_{\mathrm{CO}_{2}}=0.19 \mathrm{~L} \cdot \mathrm{s}^{-1}\right)$, as directly measured in the cell of $1.9 \mathrm{~L}$ volume, with the Dewar at $77 \mathrm{~K}$. This accurate direct measurement of pumping speed in the cell allows us to use partial pressure measurements during irradiation to quantitatively measure the photodesorption rates as a function of exposure of the ice film to radiation.

The infrared absorption spectrum of $\mathrm{CO}_{2}$ (ice) features the strong asymmetric stretching mode $\left(v_{3}\right)$. This absorption band contains inhomogeneouslybroadened LO (longitudinal) and TO (transverse) lattice phonon modes which are related to the ice crystal structure [16]. The $v_{3}$ lineshape changes slightly during irradiation, consistent with the disruption of the ice structure, and is therefore unsatisfactory for quantitative kinetic measurements. Therefore to quantitatively follow the depletion of $\mathrm{CO}_{2}$ (ice), we use the integrated absorbance of the weaker $\left(v_{1}+v_{3}\right)$ mode whose narrower lineshape does not change during irradiation. The formation of photoproducts was monitored by transmission IR spectroscopy for the ice film $[10,13]$ and by measurement of the partial pressure of desorbing gases using a QMS operating at $70 \mathrm{eV}$ electron ionization energy and using a channeltron multiplier. The relatively small cell volume and small pumping speed of our apparatus yields very high sensitivity for desorption kinetic measurements. We studied ${ }^{13} \mathrm{CO}_{2}$ (99.0\% isotopic purity) and its photoproducts in order to eliminate any possible background effect that may be caused by adsorbed ${ }^{12} \mathrm{C}$ species on the cell walls. Transmission IR spectra are acquired for 200 scans at $2 \mathrm{~cm}^{-1}$ resolution using a purged Bruker Tensor-27 IR spectrometer operating 
with an MCT detector. All the integrated IR absorbances are normalized to the initial integrated absorbance of ${ }^{13} \mathrm{CO}_{2}$ (ice) $\left(\int \mathrm{A}_{0} \mathrm{dv}\right) .{ }^{13} \mathrm{CO}_{2}$ is condensed on the $\mathrm{KBr}$ support (as well as on the reentrant Dewar in the system) from a dosing tube facing one side of the support grid. Upon irradiation of the ${ }^{13} \mathrm{CO}_{2}$ (ice) film, some scattered Lyman- $\alpha$ radiation reaches the condensed ${ }^{13} \mathrm{CO}_{2}$ (ice) layer on the Dewar surface and on the grid support clamps. $\mathrm{CO}_{2}$ (ice) on these extraneous surfaces also produces ${ }^{13} \mathrm{CO}_{2}(\mathrm{~g})$ and ${ }^{13} \mathrm{CO}(\mathrm{g})$ and other trace gaseous photoproducts sensed by the QMS. The fraction of the ${ }^{13} \mathrm{CO}(\mathrm{g})$ pressure rise caused by this effect was measured to be $70 \pm 10 \%$ by using flash heat to remove $\mathrm{CO}_{2}$ ice, and all of our analysis excludes this effect.

Using incandescent light of the same measured power density (bolometer) as the Lyman-a lamp we determined that the effects observed here are not due to heating by the lamp. The experiments at $75 \mathrm{~K}$ were checked at $82 \mathrm{~K}$. The CO desorption rate was 1.3 times as great, confirming that thermally-activated $\mathrm{CO}$ transport is mixed with photoactivation effects. The $\mathrm{CO}$ evolution is 2 orders of magnitude larger than is measured from $\mathrm{CO}_{2}$ cracking at $70 \mathrm{eV}$ ionization energy, showing that the $\mathrm{CO}$ behavior is due to $\mathrm{CO}$ desorption from the ice and not to $\mathrm{CO}_{2}$ cracking in the mass spectrometer. Care was taken using transmission IR measurements to insure that the observation of an induction period in this work was not caused by the presence of a foreign condensed layer (such as possible condensation of $\mathrm{H}_{2} \mathrm{O}$ ) on the ${ }^{13} \mathrm{CO}_{2}$ (ice) film.

\subsection{Results}

\subsubsection{Kinetics of Processes in $\mathrm{CO}_{2}$ (ice) Induced by Lyman- $\alpha$ Irradiation}

The photodissociation of ${ }^{13} \mathrm{CO}_{2}$ ice produces ${ }^{13} \mathrm{CO},{ }^{13} \mathrm{CO}_{3}$ and other trapped products, as described elsewhere [11]. Photoexcitation also produces gas phase 
$\mathrm{CO}_{2}$, $\mathrm{CO}$ and $\mathrm{O}_{2}$ molecules which are detected by the QMS. Transport of $\mathrm{O}_{2}$ is not investigated here. $\mathrm{O}_{3}$ is not detected by the mass spectrometer even though it is seen at small intensity in the IR spectra in agreement with Gerakines et al. (1996). It is found in this work that the QMS is extremely sensitive to the desorption processes, easily able to measure a desorption rate of $<10^{9}$ molecules $\cdot \mathrm{cm}^{-2} \cdot \mathrm{s}^{-1}$. In contrast transmission IR can detect the loss of about $5 \times 10^{11}$ molecules $\cdot \mathrm{cm}^{-2} \cdot \mathrm{s}^{-1}$ and is mainly an integrative measurement of changes in the bulk of the $\mathrm{CO}_{2}$ (ice) film. The QMS measurements in the gas phase are therefore highly sensitive to kinetic processes at the very outermost surface of the ice and, combined with IR measurements, provide a sensitive probe of kinetic behavior at the surface and in the bulk of the ice film during photochemistry.

Figure 5.1(a) shows the rate of production of ${ }^{13} \mathrm{CO}(\mathrm{g})$ vs. time, using the QMS. In a rapidly pumped vacuum system, the pressure measured at any time is almost exactly proportional to the rate of photodesorption (molecules $\cdot \mathrm{cm}^{-2} \cdot \mathrm{s}^{-1}$ ) at that time [17] as discussed later in eqn. 1. When the shutter is opened, the partial pressure of ${ }^{13} \mathrm{CO}(\mathrm{g})$ immediately jumped up by a small amount. This is called Process I. Process I is accompanied by a slow growth in ${ }^{13} \mathrm{CO}(\mathrm{g})$ pressure in the induction period and more rapid growth beyond called Process II. An increasingly rapid rate of evolution of ${ }^{13} \mathrm{CO}(\mathrm{g})$ is detected as Process II develops beyond the induction period. However, as shown in Figure 5.1(b) and (c), the production of trapped ${ }^{13} \mathrm{CO}$ and ${ }^{13} \mathrm{CO}_{3}$ molecules $\left[{ }^{13} \mathrm{CO}(\mathrm{Tr})\right.$ and $\left.{ }^{13} \mathrm{CO}_{3}(\mathrm{Tr})\right]$ in the matrix as measured by IR occurs by entirely different kinetics: ${ }^{13} \mathrm{CO}(\mathrm{Tr})$ and ${ }^{13} \mathrm{CO}_{3}(\mathrm{Tr})$ begin to form promptly in a process which is initially linear with radiation time, and with no observable induction period. These trapped species in the bulk ice grow to maximum mole fraction after $\sim 100$ minutes irradiation. Figure 5.1(d) shows the photodepletion of ${ }^{13} \mathrm{CO}_{2}$ (ice) as measured by IR spectroscopy. The ${ }^{13} \mathrm{CO}_{2}$ photodepletion rate is almost linear with respect to radiation time over the whole range and no significant induction period is observed. In 145 minutes of 
irradiation time, the fractional loss of ${ }^{13} \mathrm{CO}_{2}$ due to photodepletion is about $8 \%$, equivalent to $\sim 11 \mathrm{ML}$ of $\mathrm{CO}_{2}$. Because of the relative low sensitivity of the IR measurements compared to the QMS measurements, details revealed in the gas phase measurements are invisible in the IR.

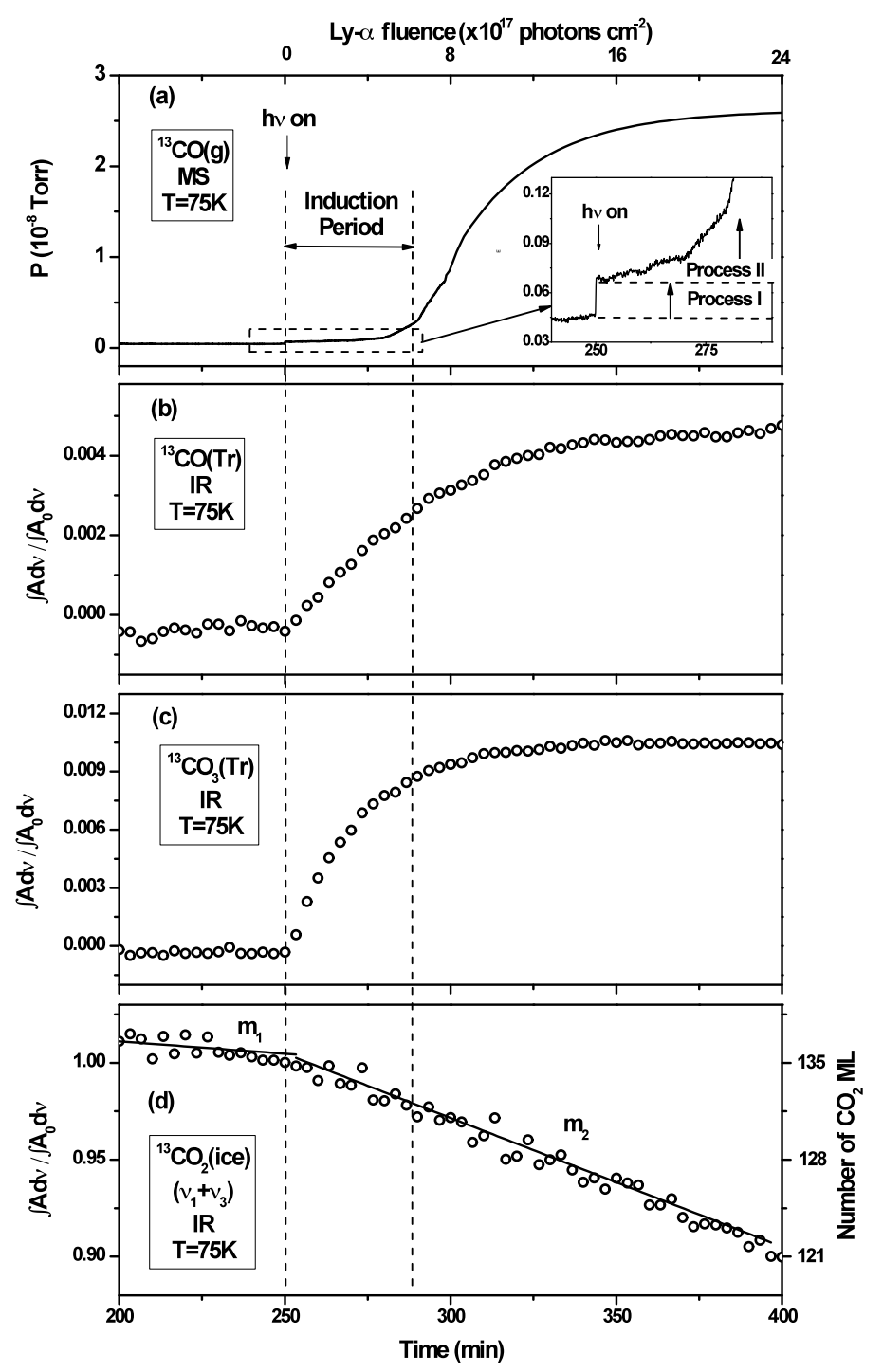

Figure 5.1. (a) Partial pressure of gas phase ${ }^{13} \mathrm{CO}$ during irradiation. The insert shows Process I and Process II with an enlarged vertical scale. The induction period corresponds to an arbitrary time where the desorption rate is a small fraction of the maximum rate. (b) ${ }^{13} \mathrm{CO}$ (Tr) formation in the ice matrix by IR spectroscopy. (c) ${ }^{13} \mathrm{CO}_{3}(\mathrm{Tr})$ 
formation in the ice matrix by IR spectroscopy. (d) Depletion of ${ }^{13} \mathrm{CO}_{2}$ (ice) by IR spectroscopy. The symbol ( $\mathrm{Tr}$ ) refers to species trapped in the ${ }^{13} \mathrm{CO}_{2}$ matrix. Under the pumping speed condition used here the pressure of ${ }^{13} \mathrm{CO}(\mathrm{g})$ is essentially proportional to the rate of desorption. Panel (d) shows that about $8 \%$ of the ice film has been lost due to photodepletion after 145 minutes irradiation. This is equivalent to the loss of about $11 \mathrm{ML}$ of ${ }^{13} \mathrm{CO}_{2}$ after correction for thermal depletion. The slope $\mathrm{m}_{1}$ is related to thermal depletion (sublimation) from the undamaged ice and the slope $m_{2}$ is due to combined photo- and thermal-depletion from the ice during exposure to Lyman- $\alpha$ radiation. The value of $\left(\mathrm{m}_{1}-\mathrm{m}_{2}\right)$ corresponds to the $\mathrm{CO}_{2}$ photodepletion rate.

The observation of a significant induction period corresponding to a photon fluence of $\sim 5.5 \times 10^{17}$ photons $\mathrm{cm}^{-2}$ at $75 \mathrm{~K} \mathrm{CO}$ (ice) temperature agrees with Öberg et al. (2009b) who observed an induction period corresponding to a fluence of $\sim 2 \times 10^{17}$ photons $\mathrm{cm}^{-2}$ at $60 \mathrm{~K}$.

The rate of desorption versus radiation time was derived from pressureversus-time data like that shown in Figure 5.1a. The evolution of $\mathrm{CO}(\mathrm{g})$ and the pumping away of the $\mathrm{CO}(\mathrm{g})$ are considered in the mass balance Equation (1).

$$
\begin{aligned}
\frac{d P(t)}{d t}= & \frac{k_{B} T}{V} \cdot A \cdot \frac{d N(t)}{d t}-\frac{S \cdot P(t)}{V} \\
& {\left[\begin{array}{l}
\text { proportional to } \\
\text { molecules evolved }
\end{array}\right] \quad\left[\begin{array}{l}
\text { proportional to } \\
\text { molecules pumped }
\end{array}\right] }
\end{aligned}
$$

where $\mathrm{P}(\mathrm{t})$ is the pressure of $\mathrm{CO}(\mathrm{g}), \mathrm{dN}(\mathrm{t}) / \mathrm{dt}$ is the number of $\mathrm{CO}$ molecules evolved per unit area and unit time, $\mathrm{S}$ is the pumping speed, $\mathrm{V}$ is the volume of the cell, $A$ is the surface area of the ice, $k_{B}$ is the Boltzmann constant, $T$ is the gas temperature in $\mathrm{K}$ and $\mathrm{t}$ is irradiation time. For the conditions of our experiment, the $\mathrm{dP}(\mathrm{t}) / \mathrm{dt}$ term is always small compared to the $\mathrm{S} \cdot \mathrm{P}(\mathrm{t}) / \mathrm{V}$ term. Thus $\mathrm{dN}(\mathrm{t}) / \mathrm{dt}$ is essentially proportional to $\mathrm{S} \cdot \mathrm{P}(\mathrm{t}) / \mathrm{N}$. 
It is observed that over the experimental period at $75 \mathrm{~K}$, a cumulative amount of only about $1 \mathrm{ML}$ of ${ }^{13} \mathrm{CO}(\mathrm{g})$ is evolved. At the same time, about $11 \mathrm{ML}$ of ${ }^{13} \mathrm{CO}_{2}$ (ice) has been photochemically destroyed as shown in Figure 5.1(d).

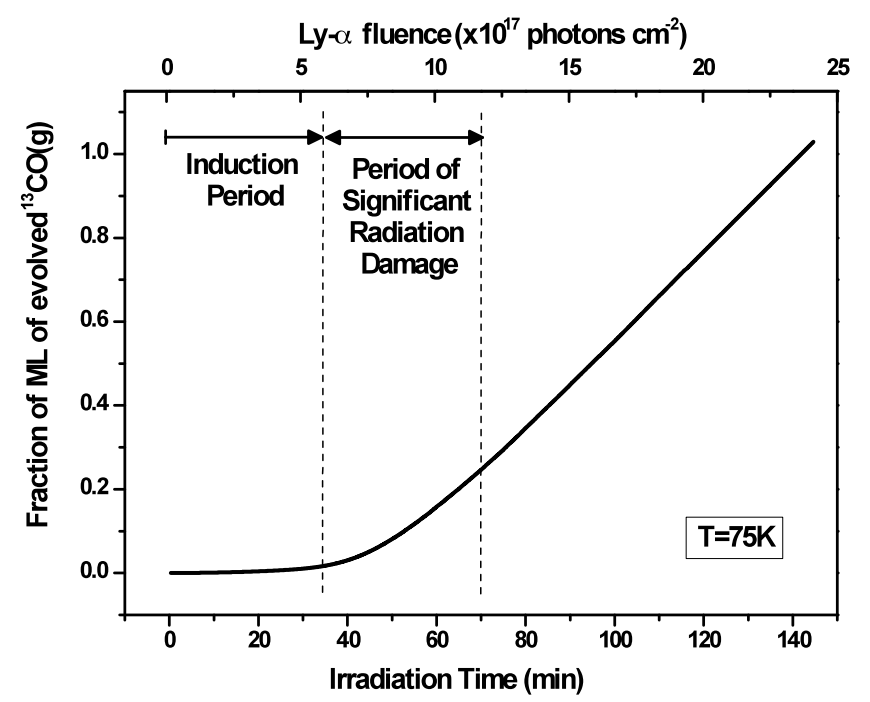

Figure 5.2. Extent of evolution of ${ }^{13} \mathrm{CO}$ into gas phase during photolysis, measured from partial pressure behavior. It is noted that only about $1 \mathrm{ML}$ of desorbing ${ }^{13} \mathrm{CO}$ is measured over the entire desorption period involving Process I and Process II. Only $0.02 \mathrm{ML}$ of ${ }^{13} \mathrm{CO}$ is evolved in the induction period. These measurements have been adjusted for photodesorption from the Dewar and support surfaces. The period of significant radiation damage corresponds to an irradiation time where rapid acceleration of photodesorption occurs as a superlinear process. In this period each $\mathrm{CO}_{2}$ (ice) molecule absorbs about 3 Lyman- $\alpha$ photons on average.

A relatively low rate of desorption of ${ }^{13} \mathrm{CO}_{2}(\mathrm{~g})$ relative to ${ }^{13} \mathrm{CO}(\mathrm{g})$, as observed by others [9], is confirmed in Figure 5.3, which compares the partial pressure of ${ }^{13} \mathrm{CO}(\mathrm{g})$ and ${ }^{13} \mathrm{CO}_{2}(\mathrm{~g})$ during irradiation. The ${ }^{13} \mathrm{CO}(\mathrm{g})$ partial pressure (and therefore its rate of desorption) after the achievement of steady state is about 30 times larger than that of ${ }^{13} \mathrm{CO}_{2}(\mathrm{~g})$. The ${ }^{13} \mathrm{CO}_{2}(\mathrm{~g})$ pressure behavior versus time of irradiation is similar to that of ${ }^{13} \mathrm{CO}(\mathrm{g})$, but the initial development of ${ }^{13} \mathrm{CO}_{2}(\mathrm{~g})$ 
pressure during the first part of Process I occurs gradually in comparison to ${ }^{13} \mathrm{CO}(\mathrm{g})$, which is evolved promptly at the beginning of Process I. Figure 5.3 shows that both ${ }^{13} \mathrm{CO}$ evolution and ${ }^{13} \mathrm{CO}_{2}$ evolution occur by means of two processes, I and II. The similarity of kinetic behavior for both desorbing gases suggests that the physical meaning of Process I and Process II applies generally to both ${ }^{13} \mathrm{CO}$ and ${ }^{13} \mathrm{CO}_{2}$ evolution.

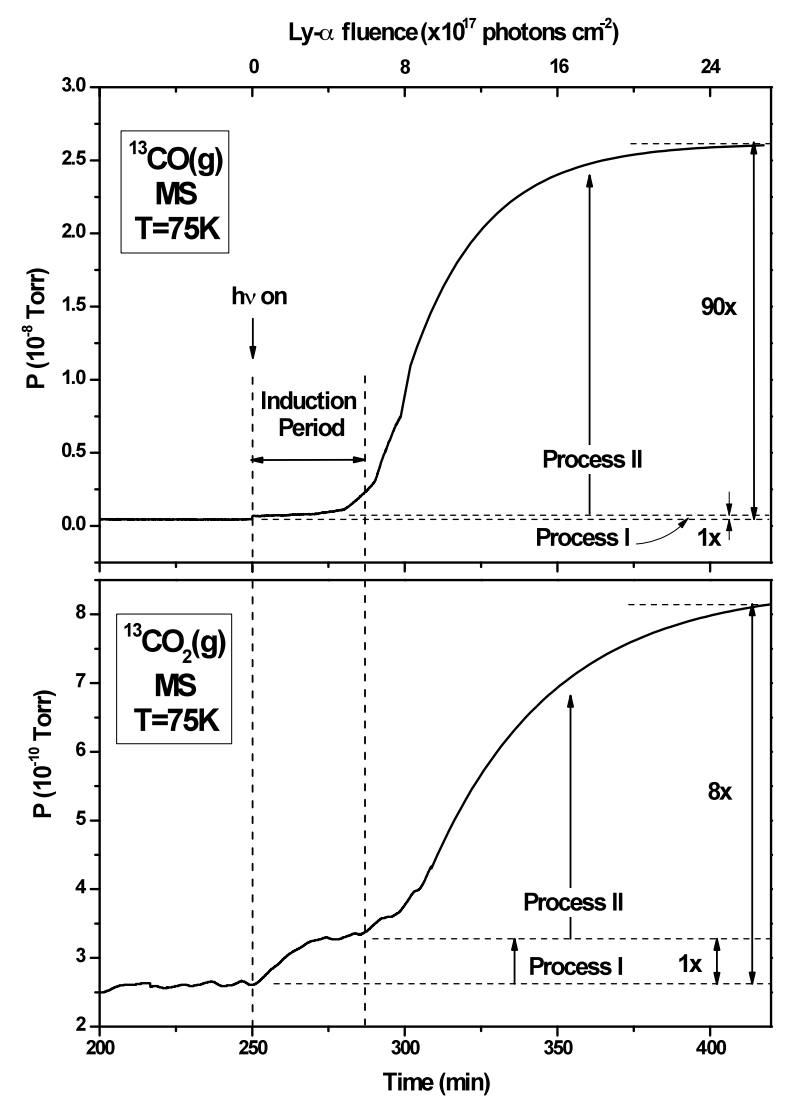

Figure 5.3. Comparison of gas phase ${ }^{13} \mathrm{CO}$ and ${ }^{13} \mathrm{CO}_{2}$ during irradiation at $75 \mathrm{~K}$.

Figure 5.4 shows the effect in annealing the ${ }^{13} \mathrm{CO}_{2}$ ice at $75 \mathrm{~K}$ for various times by interruption of the Lyman- $\alpha$ radiation. The unstippled regions indicate the annealing periods in the dark at $75 \mathrm{~K}$ for various times, $t_{1}, t_{2}$, and $t_{3}$. The growth and decay plots have been fit with exponential curves. When the Lyman- $\alpha$ 
shutter is closed, the gas phase ${ }^{13} \mathrm{CO}$ pressure (proportional to the ${ }^{13} \mathrm{CO}$ desorption rate) drops slowly by a significant fraction during each annealing period at $75 \mathrm{~K}$ when Lyman- $\alpha$ irradiation is turned off. The fact that the ${ }^{13} \mathrm{CO}$ pressure drop occurs over a long time period compared to the time constant for pumping $\left(\tau_{\text {pump }}=\mathrm{V} / \mathrm{S}=9.0 \mathrm{~s}\right.$ for $\left.\mathrm{CO}(\mathrm{g})\right)$ indicates that a thermally-activated desorption process at $75 \mathrm{~K}$ is also occurring from the radiation-damaged ice after interruption of the Lyman- $\alpha$ irradiation. In addition, recovery of the maximum photodesorption rate occurs only slowly after the shutter is opened. Meanwhile, during the annealing period the IR integrated intensity of ${ }^{13} \mathrm{CO}(\mathrm{Tr})$, shown in Figure 5.4(b), drops by only $5-10 \%$, showing that most of the produced ${ }^{13} \mathrm{CO}$ molecules are still trapped inside the ice bulk and that gas phase measurements using the QMS primarily sample the kinetics of desorption from the surface and near-surface region of the ice, as it is fed by ${ }^{13} \mathrm{CO}$ diffusion through the bulk. 


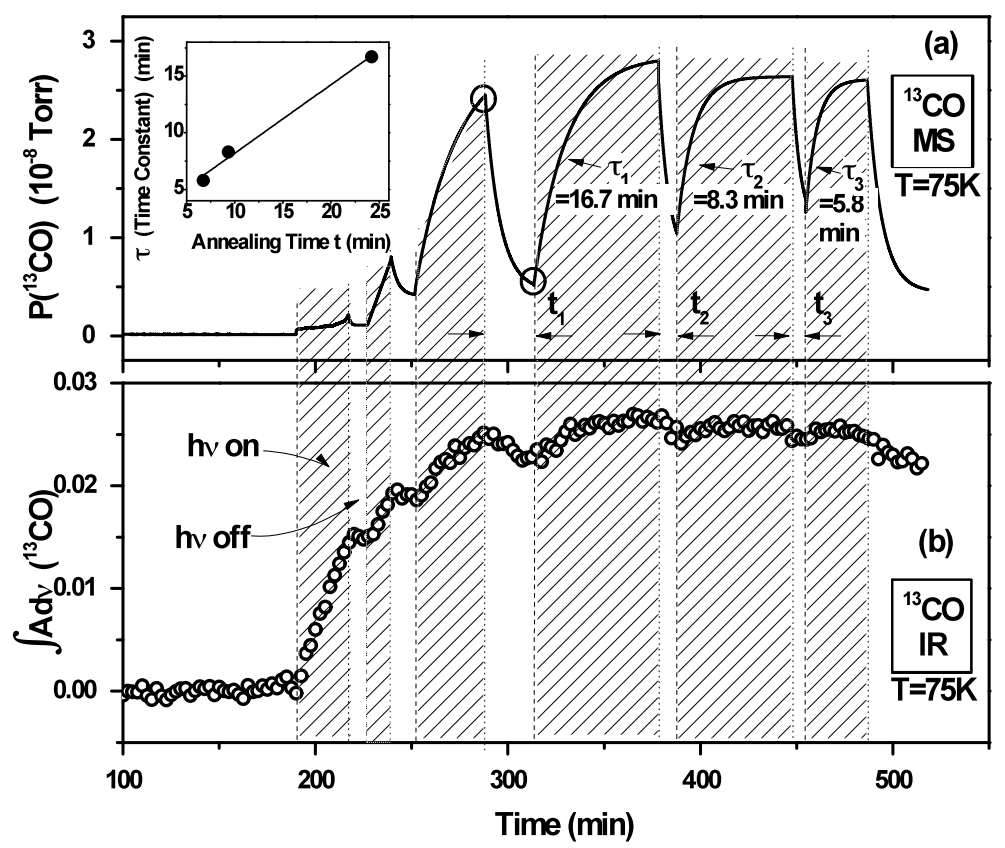

Figure 5.4. Effect of annealing at $75 \mathrm{~K}$ for various times on the rate of recovery of the ${ }^{13} \mathrm{CO}$ photodesorption rate. The shutter is open in the cross-hatched regions causing photodesorption and radiation damage to the ice, and then closed to cause ice annealing in the absence of radiation in the clear regions. Inset: Dependence of the time constant for recovery of the ${ }^{13} \mathrm{CO}$ desorption rate after various annealing times at $75 \mathrm{~K}$, indicating that longer annealing in the dark at $75 \mathrm{~K}$ reduces the rate of recovery of the ice to the fast desorption condition.

It is observed in the inset in Figure 5.4(a) that the time constant, $\tau$, for recovery of the photodesorption rate to its maximum during irradiation increases monotonically with the preannealing time at $75 \mathrm{~K}$. This clearly indicates that the radiation damage process which accelerates desorption can be partially reversed by annealing at $75 \mathrm{~K}$ in the dark. A longer irradiation time to produce more extensive damage to the $\mathrm{CO}_{2}$ (ice) leads to an enhancement of ${ }^{13} \mathrm{CO}$ desorption rate. Annealing causes a slow reduction in the ${ }^{13} \mathrm{CO}$ photodesorption rate as seen by the reduction in ${ }^{13} \mathrm{CO}$ pressure when comparing the rate of ${ }^{13} \mathrm{CO}$ evolution at the end of an irradiation period with the ${ }^{13} \mathrm{CO}$ evolution rate for the 
annealed $\mathrm{CO}_{2}$ (ice) which is achieved immediately upon turning on the Lyman- $\alpha$ radiation at the end of an annealing period. This difference may be seen for example by comparing the two circled points in Figure 5.4(a). It is also noted in Figure 5.4(b) that measurements in the bulk by transmission IR also clearly indicate that only a small fraction of trapped- ${ }^{13} \mathrm{CO}$ thermal desorption is observed in the dark during each $75 \mathrm{~K}$-annealing period. These results are indicative of the combined action in Process II of partial ${ }^{13} \mathrm{CO}(\mathrm{Tr})$ production during irradiation at $75 \mathrm{~K}$ combined with ${ }^{13} \mathrm{CO}(\mathrm{Tr})$ thermal desorption and photodesorption during irradiation at $75 \mathrm{~K}$. Thermal desorption continues when the previously irradiated and damaged ice is stored in the dark at $75 \mathrm{~K}$. Thus, ${ }^{13} \mathrm{CO}$ and ${ }^{13} \mathrm{CO}_{2}$ photodepletion at $75 \mathrm{~K}$ involves a combination of photodesorption and thermally controlled diffusion and desorption of both ${ }^{13} \mathrm{CO}$ and ${ }^{13} \mathrm{CO}_{2}$ in the damaged $\mathrm{CO}_{2}$ (ice). The efficiency of the thermal processes increases very significantly as radiation damage to the ice film increases.

\subsubsection{Spectroscopic Observations of ${ }^{13} \mathrm{CO}_{2}$ (ice) Damage by Lyman- $\alpha$ Radiation}

As shown in Figure 5.5 the strong $v_{3}$ mode of ${ }^{13} \mathrm{CO}_{2}$ (ice) displays evidence for the inhomogeneous broadening of this molecular mode with the appearance of LO $\left(2314 \mathrm{~cm}^{-1}\right)$ and TO $\left(2280 \mathrm{~cm}^{-1}\right)$ phonon modes from the lattice vibrations of the ice [16]. The lattice-related LO spectral band consists of a shoulder on the high wavenumber side of the TO-mode. The absorbance in this shoulder is related to the ${ }^{13} \mathrm{CO}_{2}$ crystallite size, crystalline shape and crystalline order in the ice as well as to changes due to $\mathrm{CO}_{2}$ depletion. When the crystallite size is made small compared to the wavelength of the IR light, the absorption is intensified near the LO lattice mode[16]. Increasing the lattice damage in the crystallites is expected to cause a decrease of the relative intensity of the absorption in the region near the LO mode since vibrational coupling between $\mathrm{CO}_{2}$ molecules will diminish in the disordered ice [16]. Figure 5.5 shows spectral changes in the $v_{3}$ 
region where spectra are shown during Lyman-a irradiation at $75 \mathrm{~K}$. It is seen that ${ }^{13} \mathrm{CO}_{2}$ photodepletion leads to losses in intensity in both the LO and TO spectral regions.

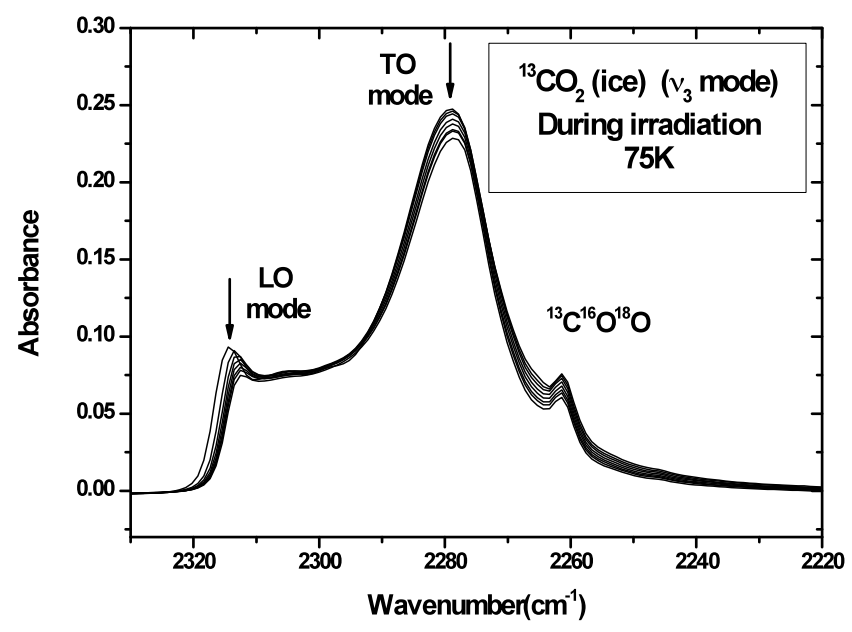

Figure 5.5. Spectral changes in the $\mathrm{v}_{3}$ region for ${ }^{13} \mathrm{CO}_{2}$ (ice) during Lyman-a irradiation at $75 \mathrm{~K}$.

Figure 5.6 shows the difference spectra in the $v_{3}$ region when the ice is first irradiated and then annealed in the dark at $75 \mathrm{~K}$. Here the difference spectra clearly show the reverse trend in absorbance for the LO mode as ice crystallinity reforms from the damaged crystal lattice upon annealing. The negative difference feature at $2314 \mathrm{~cm}^{-1}$ in Figure 5.6(a) is caused by the combination of lattice damage and $\mathrm{CO}_{2}$ depletion. As shown in Figure 5.6(b), the LO-mode $\mathrm{CO}_{2}$ absorbance at $2314 \mathrm{~cm}^{-1}$ is restored on annealing in the dark at $75 \mathrm{~K}$, while some ${ }^{13} \mathrm{CO}_{2}$ depletion by a thermally activated process at $75 \mathrm{~K}$ continues as seen by the decrease in absorbance of the $2280 \mathrm{~cm}^{-1}$ and $2274 \mathrm{~cm}^{-1}$ features. This observation indicates that the $2314 \mathrm{~cm}^{-1} \mathrm{LO}$ mode integrated absorbance may be used to observe ${ }^{13} \mathrm{CO}_{2}$ crystal damage during irradiation and that its partially reversible behavior upon annealing at $75 \mathrm{~K}$ in the dark is related to the reformation of crystalline order in the damaged ice. Thus the radiation-induced 
$\mathrm{CO}_{2}$ lattice disordering and ordering effects shown in Figure 5.4, caused by alternating radiation damage and annealing at $75 \mathrm{~K}$, are confirmed in another way in Figure 5.6 when one observes the LO phonon mode behavior, where annealing at $75 \mathrm{~K}$ leads to ordering of a photochemically-disordered lattice. A purely optical explanation of the LO-TO lineshape is given by [18] and [19], where the relation of optical properties to lattice damage in crystallites is not explicitly recognized.

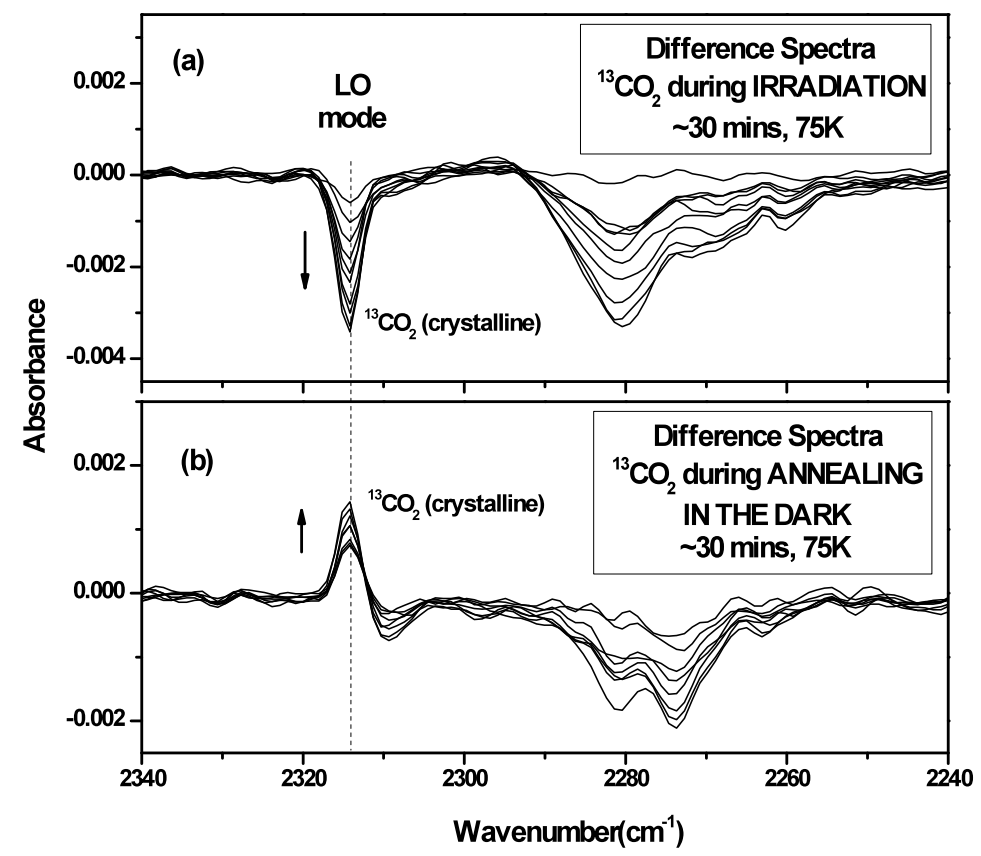

Figure 5.6. Difference spectra during irradiation of ${ }^{13} \mathrm{CO}_{2}$ (ice) followed by annealing in the dark at $75 \mathrm{~K}$.

Figure 5.7 shows the change of the $v_{2}$ bending mode spectrum of $\mathrm{CO}_{2}$ (ice) during irradiation. The $v_{2}$ mode is also a characteristic mode for distinguishing crystalline and amorphous $\mathrm{CO}_{2}$ (ice). In crystalline ${ }^{13} \mathrm{CO}_{2}$ (ice) there are two sharp bands at 641 and $636 \mathrm{~cm}^{-1}$, while amorphous ice exhibits only one broad band at around $640 \mathrm{~cm}^{-1}$ [14]. In Figure 5.7, during irradiation, the crystalline double bands decrease during irradiation, and a new band related to the 
formation of amorphous $\mathrm{CO}_{2}$ (ice) increases in between them. This observation combined with the spectra shown in Figure 5.5 and 6 show that Lyman- $\alpha$ irradiation changes crystalline $\mathrm{CO}_{2}$ (ice) into an amorphous phase. Similar radiation-induced phase transitions have also been observed in water ices [5].

From the effects shown in Figures 5, 6 and 7, it is unfortunately not possible to determine the extent of lattice damage due to Lyman- $\alpha$ radiation since the $\mathrm{CO}_{2}$ (ice) LO mode absorbances are not simply related quantitatively to the extent of damage. An isotopically layered $\mathrm{CO}_{2}$ (ice) study provides further support to the long-range damage model, as discussed below.

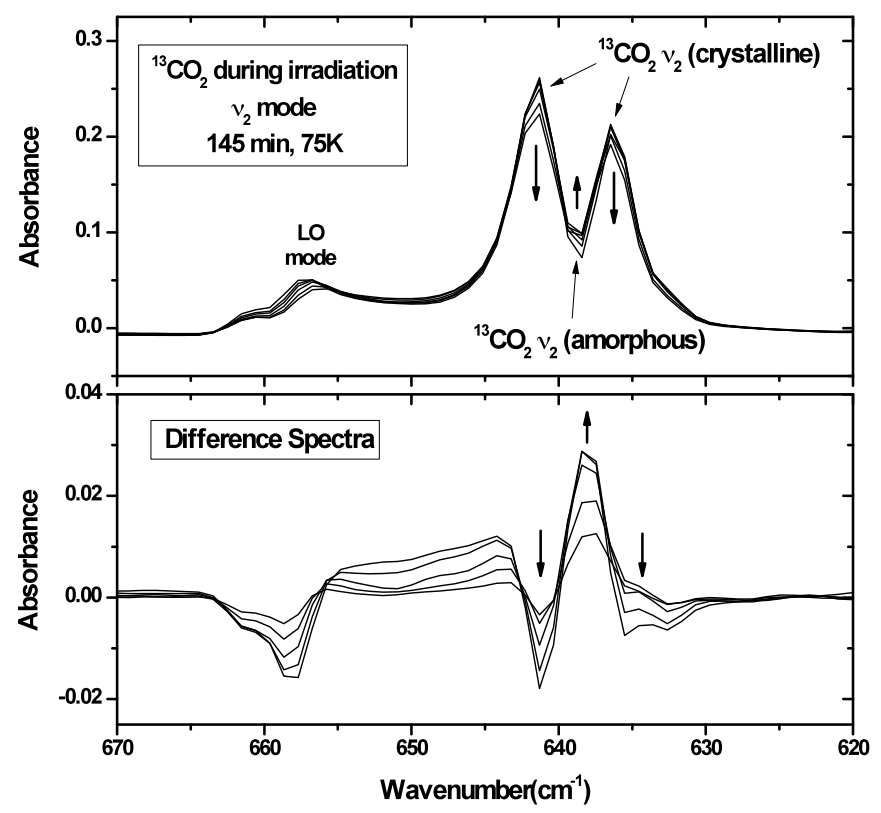

Figure 5.7. The $v_{2}$ mode of ${ }^{13} \mathrm{CO}_{2}$ (ice) during irradiation at $75 \mathrm{~K}$. The total time of irradiation is $145 \mathrm{~min}$. 


\subsubsection{Detection of the Depth of Radiation Damage in ${ }^{13} \mathrm{CO}_{2}$ (ice) by Lyman- a Radiation}

Figure 5.8 shows a schematic diagram of a sandwich structure of ${ }^{12} \mathrm{CO}_{2}$ (ice) on top of ${ }^{13} \mathrm{CO}_{2}$ (ice) made by sequential deposition of $25 \mathrm{~nm}$ of each isotopomer at $75 \mathrm{~K}$. This ice film structure was produced to test whether radiation damage of the ice will extend deeply into the film, causing the underlayer of ${ }^{13} \mathrm{CO}_{2}$ (ice) to be photodissociated and whether the ${ }^{13} \mathrm{CO}$ photoproduct made only in the underlayer will be delivered efficiently to the surface causing its desorption. Two observations in Figure 5.8 may be made for the sandwich structure: (1). Process $\mathrm{I}$ is essentially absent from the sandwich ice structure for ${ }^{13} \mathrm{CO}$ evolution as a result of the presence of a $25 \mathrm{~nm}{ }^{12} \mathrm{CO}_{2}$ capping layer; (2). Process II efficiently delivers ${ }^{13} \mathrm{CO}$ from the under layer to the gas phase for the radiation-damaged $\mathrm{CO}_{2}$ (ice). The second observation strongly indicates that deep radiation damage is occurring during Process II and that this damage extends over the $50 \mathrm{~nm}$ depth of the film, even in the early stages of Process II. 


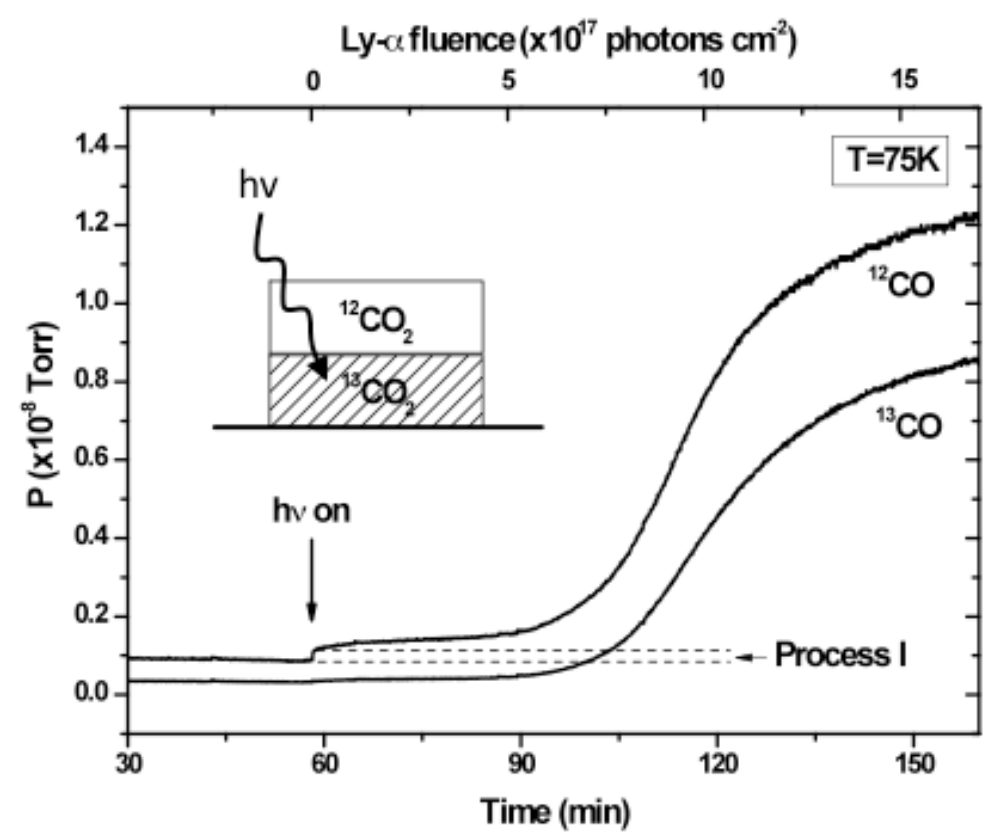

Figure 5.8. Comparison of ${ }^{12} \mathrm{CO}$ and ${ }^{13} \mathrm{CO}$ evolution from a $25 \mathrm{~nm}$ sandwich film of ${ }^{12} \mathrm{CO}_{2}$ (ice) on top of a $25 \mathrm{~nm}$ film of ${ }^{13} \mathrm{CO}_{2}$ (ice) during irradiation with Lyman-a radiation at $75 \mathrm{~K}$.

Figure 5.9 displays the mole fraction, $\mathrm{X}_{13 \mathrm{co}}$, of ${ }^{13} \mathrm{CO}$ (from the underlayer ice in the sandwich film structure) being evolved as desorbing gas during Process I and Process II. It may be seen that only a small fraction of ${ }^{13} \mathrm{CO}(\mathrm{g})$ is evolved in Process I whereas as Process II develops, the mole fraction of ${ }^{13} \mathrm{CO}$ increases significantly as radiation damage is accumulated deep in the ice film. Thus in Process II, ${ }^{13} \mathrm{CO}$ transport occurs readily throughout the thickness of the ${ }^{13} \mathrm{CO}_{2}$ (ice) film. 


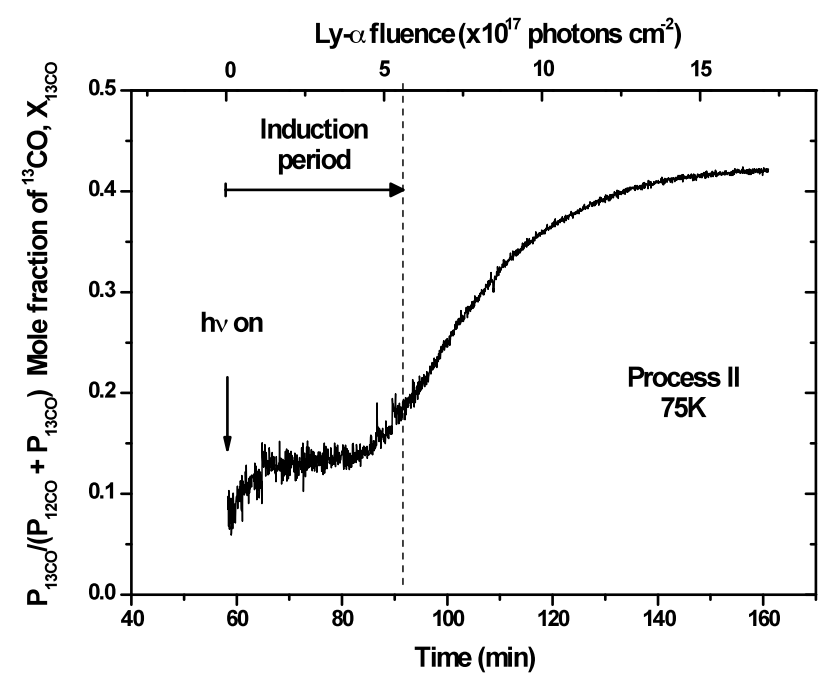

Figure 5.9. Isotopic composition of $\mathrm{CO}$ evolution in isotopic sandwich ice structure as radiation damage increases at $75 \mathrm{~K}$. The value of $\mathrm{X}_{13 c 0}$ approaches its theoretical maximum of 0.5 as deep radiation damage occurs in the $50 \mathrm{~nm} \mathrm{CO}$ (ice) film.

\subsection{Discussion of Results}

\subsection{1 $\mathrm{CO}$ and $\mathrm{CO}_{2}$ Photo- and Thermal-Desorption from $\mathrm{CO}_{2}$ (ice) at $75 \mathrm{~K}$}

Process I, shown in Figure 5.1(a) and Figure 5.3, is, for CO, a prompt process which develops to completion immediately when the $\mathrm{CO}_{2}$ (ice) surface first receives Lyman-a photons. This process is caused by the photodecomposition of $\mathrm{CO}_{2}$ molecules accompanied by fast loss of $\mathrm{CO}$ to the gas phase. The promptness of Process I and its small magnitude ( $<2 \%$ of a $M L$ evolved during the induction period) suggests that it does not involve thermallyactivated $\mathrm{CO}$ transport steps but is a direct photoprocess originating from the outermost surface-bound $\mathrm{CO}_{2}$ molecules which are photodissociated on a time scale of order $10^{-15} \mathrm{~s}$. As shown in Figure 5.8 , Process I can be significantly attenuated by the adsorption of another crystalline isotopic $\mathrm{CO}_{2}$ (ice) layer on the surface. 
When $\mathrm{CO}$ is photochemically-produced in $\mathrm{CO}_{2}$ (ice), the rate of desorption of $\mathrm{CO}$ is observed in Process II to increase as the mole fraction of trapped CO increases in the ice; Process II begins to develop (in a super-linear manner) from the beginning, as shown in the inset to Figure 5.1(a) and in Figure 5.2 where the superlinear radiation damage process continues to occur. During the initial part of Process II, the superlinear development of the CO desorption rate is accompanied only by a linear increase in the absorbance of $\mathrm{CO}(\mathrm{Tr})$ as $\mathrm{CO}_{2}$ photodecomposition occurs inside the ice (see Figure 5.1(b), initial region). Hence, we postulate that Process II involves a CO desorption process enhanced strongly by a non-linear buildup of radiation damage in the $\mathrm{CO}_{2}$ (ice). This leads to an enhancement of the rate of $\mathrm{CO}(\mathrm{g})$ evolution beyond the linear rate expected from the linear growth of $\mathrm{CO}(\mathrm{Tr})$ absorbance during photodecomposition of $\mathrm{CO}_{2}$ (ice). Öberg et al. (2009b) have also reported that the mean free path of $\mathrm{CO}$ through $\mathrm{CO}_{2}$ (ice) can be very long, corresponding to the deep radiation damage observed here.

The photoactivation of $\mathrm{CO}_{2}($ ice $)$ at $75 \mathrm{~K}$ produces both $\mathrm{CO}$ and $\mathrm{CO}_{2}$ gaseous products. At $75 \mathrm{~K}$, even in the absence Lyman-a radiation, slow thermal desorption (sublimation) of $\mathrm{CO}_{2}$ (ice) occurs (shope $=\mathrm{m}_{1}$ ) as may be seen in Figure $5.1(d)$ where a slight decrease in the $\left(v_{1}+v_{3}\right)$ mode absorbance occurs during $\mathrm{CO}_{2}$ (ice) storage in the dark at $75 \mathrm{~K}$ prior to initiating photochemistry. The observation of a superlinear behavior for $\mathrm{CO}$ during Process II may be explained by a radiation-damage process in the $\mathrm{CO}_{2}$ lattice leading to a significantly enhanced $\mathrm{CO}$ transport process through the ice and thermally-activated release from the ice surface into vacuum. A similar radiation-damage enhancement of $\mathrm{CO}_{2}(\mathrm{~g})$ desorption also occurs in Process II. 
Table I. Comparison of Lyman- $\alpha$ Photodesorption Yield with Literature Reports

\begin{tabular}{|l|l|l|l|}
\hline Reference & This work & Öberg et al. (2009b) & Bahr et al. (2012) \\
\hline Method & $\begin{array}{l}\text { Transmission } \\
\text { IR }+ \text { mass } \\
\text { spectrometry } \\
\text { measurement }\end{array}$ & $\begin{array}{l}\text { Reflection } \\
\text { Absorption IR } \\
\text { measurement + } \\
\text { QMS measurement }\end{array}$ & $\begin{array}{l}\text { Microbalance } \\
\text { measurement }\end{array}$ \\
\hline $\mathrm{CO}_{2}$ (ice) thickness & $135 \pm 10 \mathrm{ML}$ & $11 \mathrm{ML}$ & $420 \mathrm{ML}$ \\
\hline $\mathrm{h} v$ flux (photons $\left.\mathrm{cm}^{-2} \mathrm{~s}^{-1}\right)$ & $(2.7 \pm 0.7) \times 10^{14}$ & $2.3 \times 10^{13}$ & $10^{14} \sim 10^{15}$ \\
\hline Temperature & $75 \pm 0.5 \mathrm{~K}$ & $60 \mathrm{~K}$ & $60 \mathrm{~K}$ \\
\hline $\begin{array}{l}\mathrm{CO}_{2} \text { desorption yield } \\
(\text { molecule/photon) }\end{array}$ & $(1.0 \pm 0.5) \times 10^{-3}$ & $2 \times 10^{-3}$ & 0.2 \\
\hline
\end{tabular}

Footnote to Table I: The photodesorption yield is obtained by dividing the number of evolved molecules by the absorbed photon fluence. Table I compares three recent measurements of the Lyman- $\alpha$ photodesorption yield of $\mathrm{CO}_{2}$ (ice). Our measured desorption yield of $(1.0 \pm 0.3) \times 10^{-3} \mathrm{CO}_{2}$ molecule/photon (in Process II region) is in agreement with Öberg et al. (2009b) who used IR+QMS measurements, even though the ice thickness and photon flux and measurement methods are different. This yield is 100 times smaller than the microbalance measurement by Bahr et al. (2012). The reason for this discrepancy is unclear.

\subsubsection{Gas Phase Kinetic Measurements versus Bulk IR Measurements in $\mathrm{CO}_{2}$ (ice)-Separating Surface Processes from Bulk Processes}

The use of the QMS to measure gas desorption from an ice in these experiments provides about 1000 times the sensitivity to the kinetics of molecular transport from the ice surface compared to the sensitivity of transmission IR measurements through the ice. Interesting surface processes therefore become visible from the gas phase kinetics measurements which cannot be discerned by 
IR. Thus effects having to do with changes in desorption rates due to lattice radiation damage can be studied effectively by use of gas phase measurements. This is shown by the observation with the QMS of the enhancement of the CO desorption rate during Process II. As shown in Figure 5.2, the total evolution of gas phase $\mathrm{CO}$ is only about $1 \mathrm{ML}$ over the period of the experiment, whereas $\mathrm{CO}_{2}$ depletion in the bulk involves the loss of about $11 \mathrm{ML}$ of $\mathrm{CO}_{2}$. Thus, the $\mathrm{CO}_{2}$ depletion measured by IR is mainly due to photodecomposition of $\mathrm{CO}_{2}$ and not to desorption of $\mathrm{CO}$ or $\mathrm{CO}_{2}$. The desorption of $\mathrm{CO}$ is a minor process compared to $\mathrm{CO}_{2}$ photodecomposition. The desorption of $\mathrm{CO}_{2}$ is an even more minor process compared to $\mathrm{CO}_{2}$ photodecomposition. Hence, the IR measurements of $\mathrm{CO}_{2}$ behavior in the ice will have low sensitivity to the $\mathrm{CO}$ and $\mathrm{CO}_{2}$ desorption process.

\subsubsection{Deep Defect Formation in $\mathrm{CO}_{2}$ (ice) by Lyman-a Radiation- The Provision of New Transport Pathways to the Ice Surface}

The enormous enhancement of the $\mathrm{CO}$ (and $\mathrm{CO}_{2}$ ) desorption rate during Process II is related to radiation-induced lattice damage to the $\mathrm{CO}_{2}$ lattice, in which embedded $\mathrm{CO}_{2}$ molecules are photodissociated to produce excited $\mathrm{CO}$ and $\mathrm{O}$ species. These fragment species carry several $\mathrm{eV}$ of kinetic energy and cause long range damage to crystalline $\mathrm{CO}_{2}$ (ice), producing amorphous phase $\mathrm{CO}_{2}$ (ice). The conversion of crystalline $\mathrm{CO}_{2}$ (ice) to amorphous ice is postulated on the basis of the vibrational spectral changes observed as radiation damage builds up as shown in Figures 5-7. In the superlinear period each $\mathrm{CO}_{2}$ (ice) molecule has absorbed on average 3 Lyman- $\alpha$ photons. This leads one to conclude that massive ice damage is occurring in this experiment. A similar energy relaxation upon electronic excitation process has been studied by [20]. The radiation-induced lattice damage process is accompanied by lattice healing due to annealing at $75 \mathrm{~K}$, and radiation damage may therefore be reversed to some degree by annealing in the dark at $75 \mathrm{~K}$, as seen in Figure 5.5 and 5.6 as 
well as in Figure 5.4. The fact that no induction period is observed for all annealing experiments shown in Figure 5.4 (beyond the initial induction period seen in Figures 1-3) suggests that the dispersal of $\mathrm{CO}$ into the amorphous $\mathrm{CO}_{2}$ phase may reduce the reversibility of the amorphous-to-crystalline $\mathrm{CO}_{2}$ (ice) annealing process.

Figures 8 and 9 show that an isotopically layered $\mathrm{CO}_{2}$ (ice) sandwich may be used to understand the details of Process I and Process II. For Process I, Figure 5.8 shows that it occurs primarily from $\mathrm{CO}_{2}$ molecules on the outer surface of the $\mathrm{CO}_{2}$ (ice). A second layer of ${ }^{12} \mathrm{CO}_{2}$ on a ${ }^{13} \mathrm{CO}_{2}$ underlayer suppresses Process I significantly from the underlayer. However, as Process II grows in importance, the rate of delivery of ${ }^{13} \mathrm{CO}$ molecules from photodecomposition of the ${ }^{13} \mathrm{CO}_{2}$ underlayer to vacuum increases dramatically as a result of spatially extensive radiation damage to the ice crystal. This is seen in Figure 5.9 by the plot of the mole fraction of desorbing underlayer- ${ }^{13} \mathrm{CO}$ which approaches its theoretical maximum of 0.5 when extensive lattice damage has occurred on the isotopic layers of equal thickness, but when only $8 \%$ of the $\mathrm{CO}_{2}$ (ice) has been permanently dissociated.

One might suppose that long range damage causes complete spatial mixing of ${ }^{12} \mathrm{CO}_{2}$ and ${ }^{13} \mathrm{CO}_{2}$ molecules of the isotopically-layered ice, but Figure 5.10 shows that this does not occur. As lattice damage increases, measurements of the rate of loss of labeled $\mathrm{CO}_{2}$ molecules from both layers show that the $\mathrm{CO}_{2}$ loss rates remain almost constant (linear curves) over long irradiation times. Had complete place mixing of $\mathrm{CO}_{2}$ molecules occurred, the two isotopic $\mathrm{CO}_{2}$ depletion rates (slopes) would have become almost equal, since the difference in photodepletion rates due to the decrease of Lyman- $\alpha$ intensity as depth increases would have been removed. From the point of view of the labeled $\mathrm{CO}_{2}$ lattice molecules, the basic $\mathrm{CO}_{2}$ layer structure remains intact even though extensive lattice damage to produce an amorphous phase has occurred. In 
contrast, from the point of view of the more mobile $\mathrm{CO}$ molecules, the ${ }^{12} \mathrm{CO}$ and ${ }^{13} \mathrm{CO}$ concentration gradients in the amorphous $\mathrm{CO}_{2}$ (ice) phase are, through long-range rapid diffusion, quickly minimized as the deep radiation damage occurs, and their rates of desorption become nearly equal from heavily damaged ice.

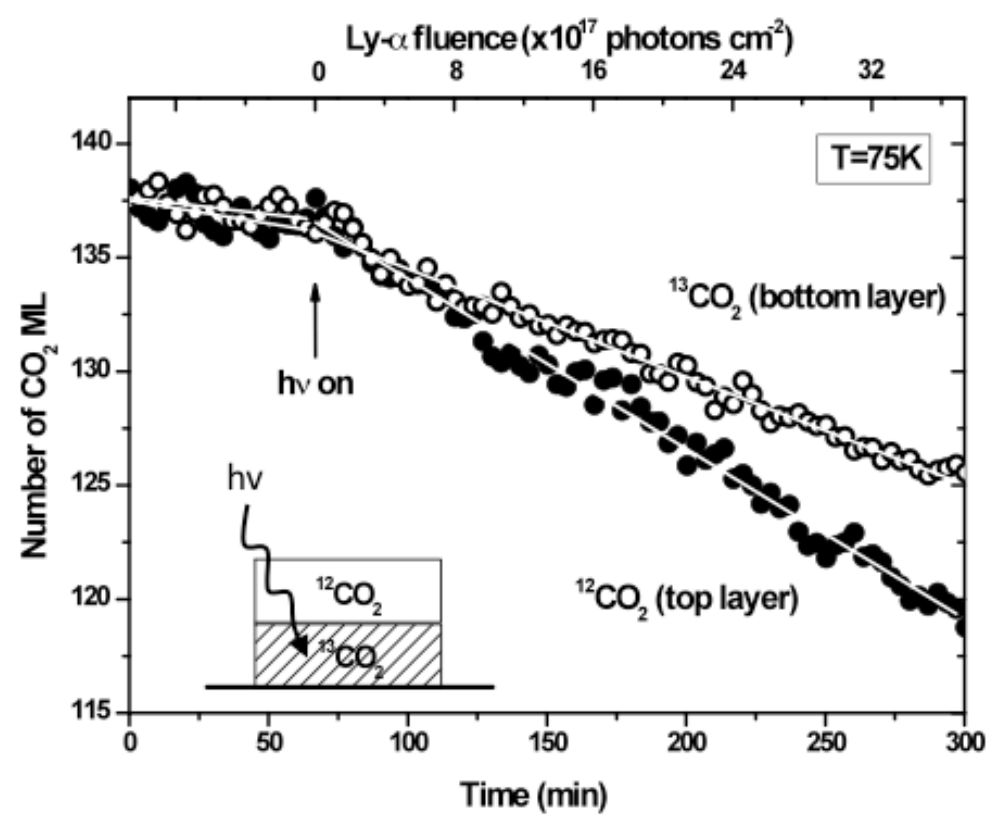

Figure 5.10. Rate of ${ }^{12} \mathrm{CO}_{2}$ and ${ }^{13} \mathrm{CO}_{2}$ depletion in a $25 \mathrm{~nm}-{ }^{12} \mathrm{CO}_{2}$ layer on top of a 25 $\mathrm{nm}-{ }^{13} \mathrm{CO}_{2}$ ice layer. Because of Lyman- $\alpha$ absorption in the ice layer the slopes differ in accordance with an absorption coefficient in $\mathrm{CO}_{2}$ (ice) of Lyman- $\alpha$ radiation of $20 \mu \mathrm{m}^{-1}$ giving a (1/e) penetration depth of $50 \mathrm{~nm}$. The continued linearity of both depletion curves is observed throughout the radiation damage time. This indicates that most isotopically-labeled $\mathrm{CO}_{2}$ (ice) lattice molecules remain localized in their own deposited layers even though extensive lattice damage has occurred throughout the depth of the ice film due to Lyman- $\alpha$ irradiation at $75 \mathrm{~K}$.

A model in which diffusing $\mathrm{CO}$ molecules sense long-range lattice damage will involve localized lattice damage processes which couple together into connected amorphous regions to give a large range for highly mobile $\mathrm{CO}$ molecules in the 
damaged region of the $\mathrm{CO}_{2}$ (ice). The coupling together of small radiationdamaged amorphous $\mathrm{CO}_{2}$ (ice) regions, to give long range transport is likely to produce superlinear transport behavior as observed for $\mathrm{CO}$ and $\mathrm{CO}_{2}$. Figure 5.11 shows a schematic diagram of $\mathrm{CO}_{2}$ (ice) in which interconnected amorphous regions are produced by radiation damage, forming spatially extensive and efficient channels for rapid $\mathrm{CO}$ transport throughout the ice thickness. As these channels penetrate the $\mathrm{CO}_{2}$ (ice) and reach the ice surface, rapid desorption of $\mathrm{CO}$ occurs from the channel ends and the $\mathrm{CO}$ desorption rate is enhanced by orders of magnitude as a result of exit channel formation, delivering mobile $\mathrm{CO}$ molecules from sites in the bulk to the surface. $\mathrm{CO}$ molecules produced by $\mathrm{CO}_{2}$ photodissociation and entering an amorphous region in the depth of the $\mathrm{CO}_{2}$ ice film will quickly transport outward through interconnected amorphous regions. The high mobility of $\mathrm{CO}$ in these regions wipes out the memory of their position upon origination by photochemical dissociation of $\mathrm{CO}_{2}$ molecules and causes them to slowly approach almost equal outward fluxes as observed (Figure 5.9) in the approach to a mole fraction of 0.5 in the desorbing ${ }^{12} \mathrm{CO}$ and ${ }^{13} \mathrm{CO}$. Since Process II behavior is also seen for desorbing $\mathrm{CO}_{2}$ molecules (Figure 5.3) and $\mathrm{O}_{2}$ molecules (not shown), the production of an amorphous $\mathrm{CO}_{2}$ phase by radiation damage in $\mathrm{CO}_{2}$ (ice) must also, to some degree, favor $\mathrm{CO}_{2}$ and $\mathrm{O}_{2}$ transport through the ice and then to vacuum. A similar effect of enhanced diffusion in amorphous solid water vs. crystalline ice has been observed, where amorphouswater ice exhibits $\sim 10^{6}$ greater $\mathrm{H}_{2} \mathrm{O}$ diffusivity than crystalline ice near $155 \mathrm{~K}$ [21].

The temperature of the phase transition from amorphous to crystalline $\mathrm{CO}_{2}$ (ice) is about $35 \mathrm{~K}$ [14]. It is likely that far below $35 \mathrm{~K}$, amorphous ice will be preserved during radiation damage, and radiation damage effects therefore will not be so spectacular in supplying rapid molecular escape pathways from the bulk to the surface. The lack of fast transport through amorphous ice at such low temperatures is probably limited by the low thermal diffusivity of both $\mathrm{CO}(\mathrm{Tr})$ and $\mathrm{CO}_{2}(\mathrm{Tr})$ at low temperatures. This is consistent with others' experimental results 
showing that the delayed onset of $\mathrm{CO}$ evolution is only observed for $\mathrm{CO}_{2}$ ices from $40-60 \mathrm{~K}$, but not observed at $18 \mathrm{~K}$ [8]. The enhancement of molecular transport through amorphous regions is attenuated at low temperatures, even though amorphous ice possesses favorable internal geometry for rapid molecular transport.

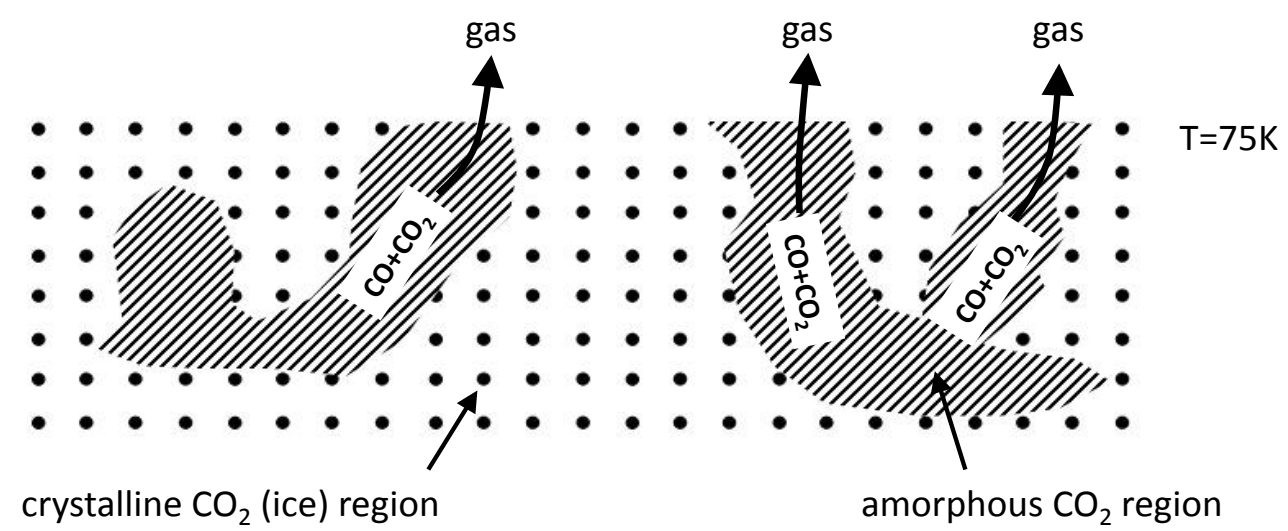

Figure 5.11. Schematic cross section of crystalline $\mathrm{CO}_{2}$ (ice) containing large interconnected radiation-induced amorphous regions which strongly favor $\mathrm{CO}$ mobility and desorption over a wide spatial range. Similar considerations apply also to $\mathrm{CO}_{2}$ molecules which enter the amorphous $\mathrm{CO}_{2}$ (ice) regions. The amorphous ice regions provide efficient exit channels for $\mathrm{CO}$ and $\mathrm{CO}_{2}$ molecules by thermally-activated transport processes at $75 \mathrm{~K}$.

\subsubsection{Synopsis of Photo Processes in Radiation-Damaged $\mathrm{CO}_{2}$ (ice)}

In the study presented here, a $\mathrm{CO}_{2}$ (ice) film of $135 \mathrm{ML}$ thickness at $75 \mathrm{~K}$ is irradiated for 145 minutes. During this time of irradiation, about $11 \mathrm{ML}$ of $\mathrm{CO}_{2}$ (ice) is destroyed and $\sim 1 \mathrm{ML}$ of $\mathrm{CO}(\mathrm{g})$ is liberated by desorption. Thus products associated with the photodissociation of $\sim 10 \mathrm{ML}$ of $\mathrm{CO}_{2}$ (ice) remain in the $\mathrm{CO}_{2}$ (ice) film, trapped as $\mathrm{CO}(\mathrm{Tr}), \mathrm{CO}_{3}(\mathrm{Tr})$ and possibly as other species. Concomitantly, only about $0.05 \mathrm{ML}$ of $\mathrm{CO}_{2}(\mathrm{~g})$ desorbs. While Process I for $\mathrm{CO}$ desorption occurs promptly, its total yield throughout the experiment is only a 
very small fraction of the $\mathrm{CO}(\mathrm{g})$ yield by Process II. The cross sections for each process are listed in Table II:

Table II. Cross Sections for Lyman-a Induced Processes in $\mathrm{CO}_{2}$ (ice) at $75 \mathrm{~K}$.

\begin{tabular}{|c|c|c|}
\hline Process & Cross section $\left(\mathrm{cm}^{2}\right)$ & Notes and references \\
\hline $\boldsymbol{\sigma}_{\text {absorption }}$ & $9 \times 10^{-18}$ & (1) \\
\hline$\sigma_{\text {excitation }}$ & $9 \times 10^{-18}$ & $\begin{array}{l}\text { Based on quantum yield in gas phase } \\
\text { (2) }\end{array}$ \\
\hline $\begin{array}{l}\sigma_{\text {total }} \mathrm{CO2} \\
\text { depletion }\end{array}$ & $(5.5 \pm 1) \times 10^{-20 *}$ & $\begin{array}{l}\text { This work; this is in agreement with our } \\
\text { previous measurement of } 5.6 \times 10^{-20} \mathrm{~cm}^{2} \\
\text { at } 75 \mathrm{~K} \text { for isotopically-pure }{ }^{13} \mathrm{CO}_{2} .(1)\end{array}$ \\
\hline$\sigma_{\mathrm{CO} \text { trapped }}$ & $(5 \pm 1) \times 10^{-20}$ * & This work \\
\hline$\sigma_{\text {CO desorption }}$ & $\begin{array}{l}(2 \pm 1) \times 10^{-22} \text { (Process I) } \\
(1 \pm 0.5) \times 10^{-20} \text { (Process } \\
\text { II) }\end{array}$ & This work \\
\hline$\sigma_{\mathrm{CO} 2 \text { desorption }}$ & $\begin{array}{l}(4 \pm 2) \times 10^{-23} \text { (Process I) } \\
(3 \pm 1.5) \times 10^{-22} \text { (Process } \\
\text { II) }\end{array}$ & $\begin{array}{l}\text { This work; this direct cross section } \\
\text { measurement for isotopically-pure } \\
\mathrm{CO}_{2} \text { (ice) is smaller than our previous } \\
\text { indirect measurement of } 1.6 \times 10^{-20} \mathrm{~cm}^{2} \\
\text { (based on analysis of the isotope } \\
\text { effect.) (3) }\end{array}$ \\
\hline
\end{tabular}

References: (1) Yuan \& Yates 2013a; (2) Slanger \& Black 1978; (3) Yuan \& Yates 2013b.

* based on average rates measured over the time period where crystalline ice is being coverted to amorphous ice in these experiments at $75 \mathrm{~K}$.

The primary finding from this work is that the desorption of $\mathrm{CO}$ and $\mathrm{CO}_{2}$ from the ice is strongly enhanced by the ice conversion from crystalline ice to amorphous ice by radiation damage. Since the photodissociation of $\mathrm{CO}_{2}$ 
molecules is the primary photochemical process occurring in the ice, it is likely that kinetically-excited $\mathrm{CO}$ and $\mathrm{O}$ product molecules within the ice result in the destruction of the nearby crystalline phase and the formation of an amorphous phase. The observation that $\mathrm{CO}$ and $\mathrm{CO}_{2}$ transport occurs over large distances during the early stage of the radiation damage process suggests that the amorphous regions of $\mathrm{CO}_{2}$ quickly coalesce at $75 \mathrm{~K}$ to make structures which extend $50 \mathrm{~nm}$ or more through the ice, providing facile transport channels for the $\mathrm{CO}, \mathrm{O}$ and $\mathrm{CO}_{2}$ species. Desorption from the emerging outer surfaces of the amorphous regions is rapid and largely governed by facile thermal transport processes through the amorphous ice as also observed by Bahr et al. (2012). The amorphous regions, as they connect to the external ice surface and to vacuum, provide "portholes" for efficient desorption as schematically shown in Figure 5.10. The molecular transport process in the ice, connecting to an amorphous-type surface desorption site, is fed by thermally-activated diffusion mainly through amorphous $\mathrm{CO}_{2}$ (ice), which provides a greatly enhanced bulk diffusion coefficient for both $\mathrm{CO}$ and $\mathrm{CO}_{2}$ molecules. The transporting molecules are initially present in the amorphous ice. A fraction may also transport from the crystalline phase across interior boundaries joining the amorphous phase to the crystalline phase. Cross sections involving molecular transport and desorption are temperature and medium dependent, with transport through crystalline material being much slower than through amorphous material in agreement with similar conclusions for enhanced transport of $\mathrm{H}_{2} \mathrm{O}$ molecules through amorphous ice compared to crystalline ice [21]. The long mean free path observed for $\mathrm{CO}$ in radiation-damaged $\mathrm{CO}_{2}$ (ice) by Öberg et al. (2009b) is also consistent with this concept. It is therefore likely that the main property governing photodesorption at higher temperatures in astronomical ices will be rapid-thermally-activated diffusion through the amorphous ice phase produced by radiation damage. 


\subsubsection{Astronomical Implications}

The photodesorption of molecules from ices in interstellar clouds is considered to be an important process in the understanding of the gas phase abundance of molecules such as $\mathrm{CO}_{2}, \mathrm{CO}$, and $\mathrm{H}_{2} \mathrm{O}[8,22,23]$. We have found that amorphous $\mathrm{CO}_{2}$ ice, produced by radiation damage, can exhibit a strongly enhanced photodesorption yield compared to crystalline ice. The UV fluence required to reach a significant level of damage in the crystalline $\mathrm{CO}_{2}$ ice is $\sim 5.5 \times$ $10^{17}$ photons $\mathrm{cm}^{-2}$, corresponding to $\sim 10^{6}$ years in typical clouds surrounding cores [24]. In relatively hot cores, it will take only hundreds of years for the radiation damage effect to occur. For other ices it is expected that similar radiation damage effects seen for $\mathrm{CO}_{2}$ ice will be discovered.

\subsection{Summary of Results}

1. For $\mathrm{CO}_{2}$ (ice) at $75 \mathrm{~K}$, an induction period for the development of the full $\mathrm{CO}$ photo-desorption rate is observed. Here mainly $\mathrm{CO}_{2}$ (ice) photodissociation from surface $\mathrm{CO}_{2}$ molecules occurs, yielding $\mathrm{CO}(\mathrm{g})$ and $\mathrm{O}(\mathrm{g})$ by a prompt process (Process I).

2. As radiation damage in the $\mathrm{CO}_{2}$ (ice) develops, a rapid $\mathrm{CO}$ photodesorption process (Process II) is observed to develop and at $75 \mathrm{~K}$ to increase the rate of $\mathrm{CO}$ desorption by about 90 -fold over Process I.

3. A similar division of the photodesorption processes for $\mathrm{CO}_{2}$ is seen, with Process I preceeding Process II.

4. The great enhancement of the $\mathrm{CO}$ and $\mathrm{CO}_{2}$ desorption rate from the $\mathrm{CO}_{2}$ (ice) surface in Process II is shown to be due to radiation damage deep in the $\mathrm{CO}_{2}$ (ice). Amorphous $\mathrm{CO}_{2}$ (ice) production, leading to an interconnected amorphous phase, provides extremely facile pathways for mobile $\mathrm{CO}$ and $\mathrm{CO}_{2}$ molecules to exit from the surface, compared to transport through crystalline ice. 
5. Lattice damage by radiation is partially reversible by annealing at $75 \mathrm{~K}$.

6. Photodesorption of $\mathrm{CO}$ and $\mathrm{CO}_{2}$ should be regarded as a combination of a photoprocess which feeds thermal transport through $\mathrm{CO}_{2}$ (ice) at $75 \mathrm{~K}$.

7. Lattice damage by radiation can be observed by measuring the intensity of the LO lattice mode seen as a shoulder on the high frequency edge of the $\mathrm{v}_{3}-\mathrm{CO}_{2}$ stretching mode. Lattice damage reduces the LO mode intensity due to the negative role of disorder in lattice coupling in the ice. Observation of the line shape behavior of the $v_{2}$ mode during irradiation and annealing also supports the concept of amorphous $\mathrm{CO}_{2}$ (ice) formation by radiation damage.

8. By using the measurement of gas desorption kinetics, and comparison to spectroscopic measurements of the behavior of molecules in the interior of the ice during irradiation, it is possible to sensitively delineate radiation-induced lattice damage processes leading to facile bulk transport and leading to desorption of both $\mathrm{CO}$ and $\mathrm{CO}_{2}$. The radiation damage process is almost invisible if only bulk kinetic measurements are made.

\subsection{References:}

1. Willacy, K. and W.D. Langer, The Importance of Photoprocessing in Protoplanetary Disks. Astrophys. J., 2000. 544: p. 903.

2. Öberg, K.I., et al., Photodesorption of CO ice. Astrophys. J., 2007. 662: p. L23.

3. Hogerheijde, M.R., et al., Detection of the Water Reservoir in a Forming Planetary System. Science, 2011. 334: p. 338.

4. Caselli, P., et al., First Detection of Water Vapor In a Pre-Stellar Core. Astrophys. J., 2012. 759: p. L37.

5. Leto, G. and G.A. Baratta, Ly-a photon induced amorphization of Ic water ice at 16 Kelvin. Astron. Astrophys., 2003. 397: p. 7. 
6. Palumbo, M.E., Formation of compact solid water after ion irradiation at $15 \mathrm{~K}$. Astron. Astrophys., 2006. 453: p. 903.

7. Raut, U., et al., Cosmic Ray Compaction of Porous Interstellar Ices. Astrophys. J., 2008. 687: p. 1070.

8. Oberg, K.I., E.F. van Dishoeck, and H. Linnartz, Photodesorption of ices I: CO, $\mathrm{N}_{2}$, and $\mathrm{CO}_{2}$. Astron. Astrophys., 2009. 496: p. 281.

9. Bahr, D.A. and R.A. Baragiola, Photodesorption of solid $\mathrm{CO}_{2}$ by Lya. Astrophys. J., 2012. 761: p. 36.

10. Rajappan, M., C. Yuan, and J.T. Yates, Jr., Lyman-a driven molecule formation on $\mathrm{SiO}_{2}$ surfaces - connection to astrochemistry on dust grains in the interstellar medium. J. Chem. Phys., 2011. 134: p. 064315.

11. Yuan, C. and J.T. Yates, Jr., Isotope Effect in the Photochemical Decomposition of $\mathrm{CO}_{2}$ (ice) by Lyman-a Radiation. J. Chem. Phys., 2013. 138: p. 154302.

12. Yuan, C. and J.T. Yates, Jr., Lyman-a Photodesorption from $\mathrm{CO}_{2}$ (ice) at $75 \mathrm{~K}$ : Role of $\mathrm{CO}_{2}$ Vibrational Relaxation on Desorption Rate. J. Chem. Phys., 2013. 138: p. 154303.

13. Rajappan, M., et al., Photochemical Decomposition of $\mathrm{N}_{2} \mathrm{O}$ by Lyman- $\mathrm{a}$ Radiation: Scientific Basis for a Chemical Actinometer. J. Phys. Chem. A, 2010. 114: p. 3443.

14. Falk, M., Amorphous solid carbon dioxide. J. Chem. Phys., 1987. 86: p. 560.

15. Xu, J., H.J. Jansch, and J.T. Yates, Jr., Cryogenic trick for enhanced cooling using liquid nitrogen. J. Vac. Sci. Technol. A, 1993. 11: p. 726.

16. Ovchinnikov, M.A. and C.A. Wight, Inhomogeneous broadening of infrared and Raman spectral bands of amorphous and polycrystalline thin films. J. Chem. Phys., 1993. 99: p. 3374.

17. Yates, J.T., Jr., The Thermal Desorption of Adsorbed Species, in Methods of Experimental Physics: Solid State Physics: Surfaces, R.L. Park and M.G. Lagally, Editors. 1985, Academic Press, Inc. p. 425.

18. Baratta, G.A. and M.E. Palumbo, Infrared optical constants of $\mathrm{CO}$ and $\mathrm{CO}_{2}$ thin icy films. J. Opt. Soc. Am. A, 1998. 15: p. 3076.

19. Palumbo, M.E., et al., The profile of the $2140 \mathrm{~cm}-1$ solid $\mathrm{CO}$ band on different substrates. Phys. Chem. Chem. Phys., 2006. 8: p. 279. 
20. Bertin, M., et al., UV photodesorption of interstellar $\mathrm{CO}$ ice analogues: from subsurface excitation to surface desorption. Phys. Chem. Chem. Phys., 2012. 14: p. 9929.

21. Smith, R.S., C. Huang, and B.D. Kay, Evidence for Molecular Translational Diffusion during the Crystallization of Amorphous Solid Water. J. Phys. Chem. B, 1997. 101: p. 6123.

22. Oberg, K.I., et al., Photodesorption of ices II: $\mathrm{H}_{2} \mathrm{O}$ and $\mathrm{D}_{2} \mathrm{O}$. Astrophys. J., 2009. 693: p. 1209.

23. Hassel, G.E., E. Herbst, and E.A. Bergin, Beyond the pseudo-time-dependent approach: chemical models of dense core precursors. Astron. Astrophys., 2010. 515: p. A66.

24. Gerakines, P.A., W.A. Schutte, and P. Ehrenfreund, Ultraviolet processing of interstellar ice analogs. I. Pure ices. Astron. Astrophys., 1996. 312: p. 289. 


\section{Chapter 6: Lyman-a Driven Molecule Formation on $\mathrm{SiO}_{2}$ Surfaces-Connection to Astrochemistry on Dust Grains in the Interstellar Medium.}

Modified from manuscript published on J. Chem. Phys. 134, 064315 (2011)

\subsection{Introduction}

It is generally accepted that the combination of molecular fragments, made by photodecomposition of parent molecules, will occur preferentially on surfaces (compared to the gas phase) to produce more complex molecules. Thus, laboratory experiments involving active species generation in the gas phase by photochemical and other methods must be done under conditions to minimize wall effects[1] inside of reaction cells, and empirical methods to reduce wall effects are widespread. On grain surfaces in the interstellar medium (ISM) it is generally accepted that surface reactions can also influence the composition on the surface as well as the very low density gas phase in contact with the grain surfaces[2-5]. The experiment to be described here compares a photochemical process in the gas phase to the same process within the pores of a silica powder containing adsorbed molecules, where surface effects will be highly amplified compared to those in the gas phase. We report that more complex products from photochemically generated radical fragment combination reactions are selectively enhanced by the proximity of the silica surface.

Experimental evidence for enhanced combination kinetics for $\mathrm{H}$ atoms near $10 \mathrm{~K}$ on silicate and graphite surfaces has been obtained by Vidali, et al.[3-5], and surface combination of $\mathrm{H}$ atoms is deemed to be responsible for the high $\mathrm{H}_{2}$ abundance found in the interstellar medium[6,7], where $\mathrm{H}_{2}$ destruction by cosmic rays in the gas phase competes with $\mathrm{H}_{2}$ formation on surfaces. Fragment 
combination processes (for $\mathrm{H}$ atoms and to some extent for other molecular fragments) in the dilute gas phase are expected to be very slow due to the need for a third body to carry off excess energy generated when bonds form between fragment species to produce more complex combination products. At low gas densities, radiative association reactions may dominate in producing combination products[8]. Alternatively, ion-molecule reactions in the gas phase have been proposed as an additional source of complex molecules in the interstellar medium[9-11].

Kinetic studies of the photodecomposition and photodesorption of ice layers made mostly from organic ices have been carried out using reflection IR spectroscopy and temperature programmed desorption by Oberg[12, 13] and by Watanabe[14, 15]. These measurements relate to surface chemistry on icecovered grains at temperatures near $10 \mathrm{~K}$ where molecular condensation into ices predominates. The work to be reported here relates to interstellar grains at higher temperatures, where ices will have evaporated leaving adsorbed species behind.

Despite the belief that surfaces promote molecular fragment combination, there are no experimental reports quantitatively comparing molecular fragment combination rates on surfaces to rates in the gas phase for any species other than atomic $\mathrm{H}[3-5]$.

The experiment to be described here involves the photodissociation of adsorbed $\mathrm{N}_{2} \mathrm{O}$ at $71 \mathrm{~K}$ followed by the combination of the photo-fragments to produce other nitrogen oxides, such as $\mathrm{NO}_{2}$ and $\mathrm{N}_{2} \mathrm{O}_{4}$. The $\mathrm{N}_{2} \mathrm{O}$ molecule is one of the approximately 160 molecular species identified at the present time in the interstellar medium $[16,17]$ and serves as a model for other more complex molecules of astrochemical importance. The gas phase photochemistry of $\mathrm{N}_{2} \mathrm{O}$ has been well studied [18-22] using Lyman-a (10.2 eV) radiation, and this work forms a basis for understanding a portion of its photochemistry in the interstellar 
medium. The primary photodissociation process can occur in the $\mathrm{N}_{2} \mathrm{O}$ molecule either at the N-O bond or at the N-N bond as shown in equations (1) and (2), below:

$$
\begin{aligned}
& \mathrm{N}_{2} \mathrm{O}+\mathrm{hv}(10.2 \mathrm{eV}) \rightarrow \mathrm{N}_{2}+\mathrm{O} \\
& \mathrm{N}_{2} \mathrm{O}+\mathrm{hv}(10.2 \mathrm{eV}) \rightarrow \mathrm{NO}+\mathrm{N}
\end{aligned}
$$

The fragment species produced photochemically may exist in different electronic states.

Fragments from the primary photochemical process may undergo a number of reactions with $\mathrm{N}_{2} \mathrm{O}$, or with each other, to produce secondary products such as $\mathrm{N}_{2}, \mathrm{O}_{2}, \mathrm{NO}, \mathrm{NO}_{2}$ and $\mathrm{N}_{2} \mathrm{O}_{4}$. We term these products "combination products". There are two types of combination reactions: those involving a radical species combining with the parent $\mathrm{N}_{2} \mathrm{O}$ molecule, and those involving the combination of two radical species. We note that $\mathrm{NO}$ is both a primary photodecomposition product as well as a combination product. A sequence of combination steps is shown in equations (3)-(7).

$$
\begin{aligned}
& \mathrm{O}+\mathrm{N}_{2} \mathrm{O} \rightarrow \mathrm{N}_{2}+\mathrm{O}_{2} \\
& \mathrm{O}+\mathrm{N}_{2} \mathrm{O} \rightarrow 2 \mathrm{NO} \\
& \mathrm{NO}+\mathrm{O} \rightarrow \mathrm{NO}_{2} \\
& \mathrm{NO}_{2}+\mathrm{O} \rightarrow \mathrm{NO}_{3} \\
& \mathrm{NO}_{2}+\mathrm{NO}_{2} \rightarrow \mathrm{N}_{2} \mathrm{O}_{4}
\end{aligned}
$$

Because of the role of silicate surfaces (dust grains) in chemistry in the interstellar medium[23, 24], we have chosen to study the kinetics and selectivity of $\mathrm{N}_{2} \mathrm{O}$ photochemistry (Lyman-a driven) on amorphous $\mathrm{SiO}_{2}$ surfaces. The high $\mathrm{SiO}_{2}$ surface area (Aerosil 200 - Degussa: $200 \mathrm{~m}^{2} / \mathrm{g}$ ) ensures that a large number of adsorbed $\mathrm{N}_{2} \mathrm{O}$ molecules are observed giving high spectroscopic sensitivity. Transmission IR spectroscopy is employed to follow the $\mathrm{N}_{2} \mathrm{O}$ decomposition as 
well as the development of combination products on the $\mathrm{SiO}_{2}$ surface. Using exactly the same ultrahigh vacuum cell, infrared spectrometer and Lyman-a lamp, we compare the $\mathrm{N}_{2} \mathrm{O} / \mathrm{SiO}_{2}$ studies with $\mathrm{N}_{2} \mathrm{O}$ gas phase photodecomposition at 10 Torr pressure. It is shown that the relative fragment combination rates (compared to $\mathrm{N}_{2} \mathrm{O}$ photo-depletion rates) are greater on $\mathrm{SiO}_{2}$ surfaces than in the gas phase, suggesting that the role of surfaces in promoting the synthesis of complex molecules in the astrochemical environment can be significant.

\subsection{Experimental}

\subsubsection{Lyman- $\alpha$ flux at $\mathrm{SiO}_{2}$ sample}

Figure 6.1 shows the quartz Lyman- $\alpha$ lamp coupled to a bakeable ultrahigh vacuum infrared cell via a $\mathrm{MgF}_{2}$ window. The Lyman- $\alpha$ lamp is sealed to the window by a Viton O-ring. The base pressure in the $1.9 \mathrm{~L}$ bakeable stainless steel cell is $1 \times 10^{-8}$ Torr during high speed pumping with both a turbo pump and an ion pump. The Lyman- $\alpha$ lamp has been calibrated using $\mathrm{N}_{2} \mathrm{O}(\mathrm{g})$ as an actinometer and the details of the lamp design, calibration and operation are given elsewhere[25]. The Lyman- $\alpha$ flux into the IR cell at the $1 \mathrm{~cm}^{2} \mathrm{MgF}_{2}$ window is $1.3 \times 10^{15}$ photons $\mathrm{s}^{-1} \mathrm{~cm}^{-2}$ and has been shown to be constant to a few percent over more than 100 hours of operation. The Lyman- $\alpha$ light flux at the $\mathrm{SiO}_{2}$ sample will be lower than measured near the $\mathrm{MgF}_{2}$ window using $\mathrm{N}_{2} \mathrm{O}$ actinometry. A thermopile with a high angular acceptance angle was placed inside the cell shown in Figure 6.2 to measure the spatial distribution of radiation emitted by the lamp at the sample position. Experiments were done in pure flowing $\mathrm{N}_{2}$ at $1 \mathrm{~atm}$. An approximately $\cos ^{300} \theta$ empirical angular distribution was measured in the vertical plane, and the light distribution in the horizontal plane was similar. We assume that the Lyman- $\alpha$ radiation exhibits a similar angular distribution to that of the mixture of wavelengths produced by the discharge in the 
$\operatorname{Ar}(90 \%)-\mathrm{H}_{2}(10 \%)$ mixture. As shown in Figure 6.2, the distribution of radiation is extremely forward focused. This directionality is due to light focusing in both the lamp and inside the cell as a result of reflections at interior surfaces. From the wide plasma source region, examples of ray tracing of reflected light to the sample are shown in Figure 6.2. The reflected radiation enhances lamp intensity at the sample. The empirical $\cos ^{300} \theta$ function includes significant contributions from inner reflections in the apparatus. The $\mathrm{SiO}_{2}$ sample if normal to the Lyman- $\alpha$ source, would receive $1.16 \times 10^{14}$ photons $s^{-1}$ within the angular distribution measured. Since for all photochemical measurements, the sample is mounted at $45^{\circ}$ to both the Lyman- $\alpha$ source and the IR beam, the corrected Lyman- $\alpha$ intensity received by the sample is $0.82 \times 10^{14}$ photons $\mathrm{s}^{-1}$. The flux of Lyman- $\alpha$ radiation at the $0.3 \mathrm{~cm}^{2}$ sample is therefore $2.7 \times 10^{14}$ photons $\mathrm{s}^{-1} \mathrm{~cm}^{-2}$. An internal shutter allows the Lyman- $\alpha$ radiation to be turned on and off. Color center formation in the $\mathrm{MgF}_{2}$ window is not observed after more than 100 hours of operation.

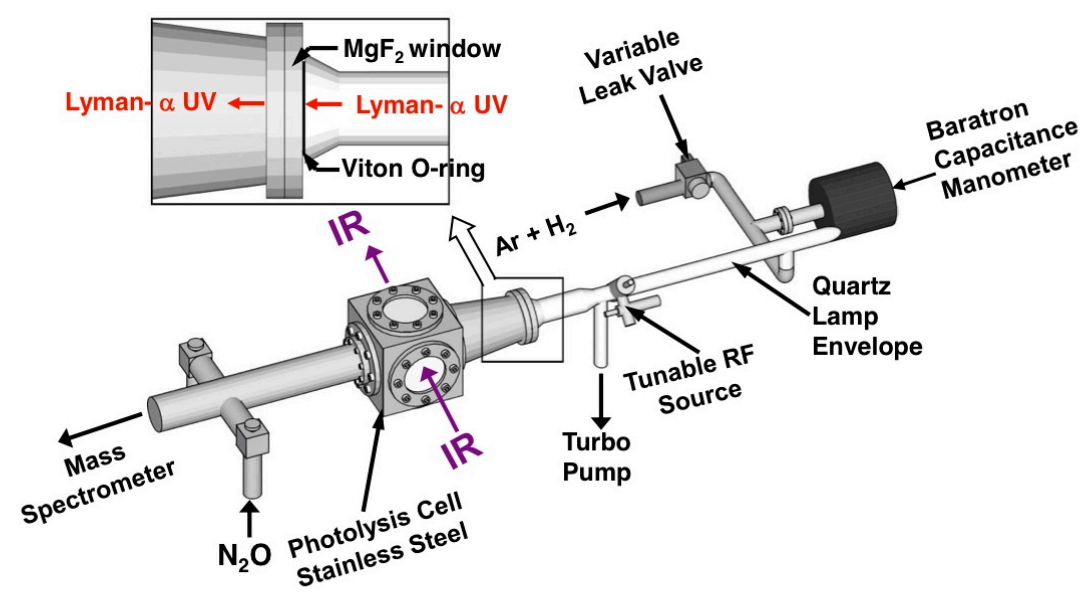

Figure 6.1: Quartz Lyman- $\alpha$ lamp coupled to the infrared cell via a $\mathrm{MgF}_{2}$ window. The QMS is located as shown. 


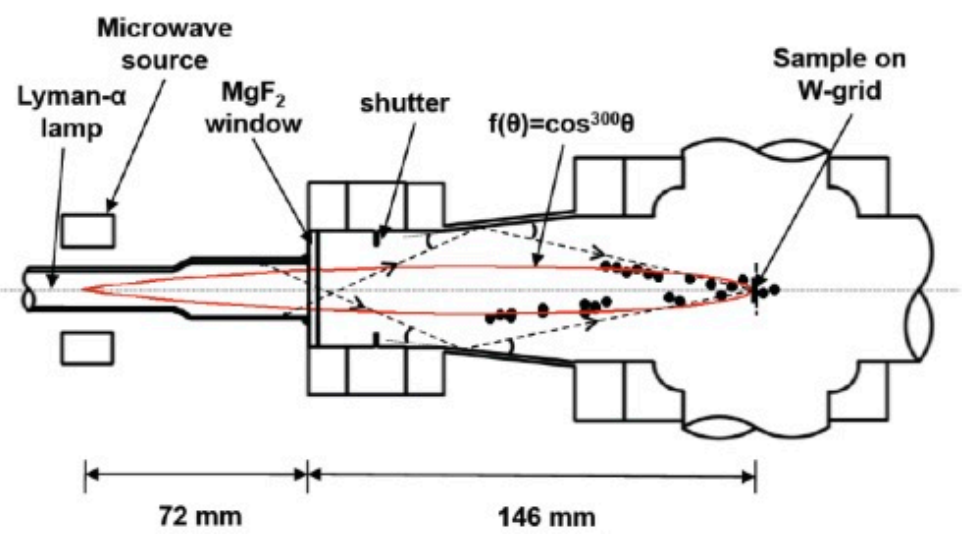

Figure 6.2: Measured distribution of emitted power from Lyman- $\alpha$ source using a thermopile. The distribution shown by the black points is very strongly peaked in the forward direction and estimated to be $\cos ^{300} \theta$, as shown by the red angular distribution.

\subsection{2 $\mathrm{SiO}_{2}$ Sample Preparation and Mounting}

As shown in Figure 6.3, the $\mathrm{SiO}_{2}$ sample (Aerosil 200, containing $2.8 \mathrm{Si}-\mathrm{OH}$ groups per $\mathrm{nm}^{2}$ of surface area)[26] is pressed into a tungsten grid $(0.005 \mathrm{~cm}$ thick) which has $22 \mu \mathrm{m} \times 22 \mu \mathrm{m}$ openings and which passes about $60 \%$ of the incident IR radiation. A $0.7 \mathrm{~cm}$ diameter $\mathrm{SiO}_{2}$ spot (containing $0.3 \mathrm{~cm}^{2}$ geometrical area of powder in the grid opening) is pressed into the grid openings. The thickness of the $\mathrm{SiO}_{2}$ layer in the grid openings is $0.005 \mathrm{~cm}$. The $\mathrm{SiO}_{2}$ weighs $2.2 \times 10^{-3} \mathrm{~g}$ and therefore exposes a surface area of $4400 \mathrm{~cm}^{2}$. The tungsten grid is stretched between two Ni clamps which may be cooled by $\mathrm{I}-\mathrm{N}_{2}$ contained inside a central reentrant rotatable Dewar. Grid temperatures of $71 \mathrm{~K}$ may be achieved by bubbling a fast stream of $\mathrm{He}(\mathrm{g})$ through the $\mathrm{I}-\mathrm{N}_{2}$ as described elsewhere[27]. The grid may be heated electrically and the temperature program may be controlled electronically by a Labview program to $0.1 \mathrm{~K}$ in the range $\sim 100 \mathrm{~K}-\sim 1000 \mathrm{~K}$ using a type $\mathrm{K}$ thermocouple welded to the grid. Should incident radiation cause small heating of the grid, the feedback 
circuit will adjust the applied electrical grid power downward to achieve a stable set point temperature in about $2 \mathrm{~s}$.

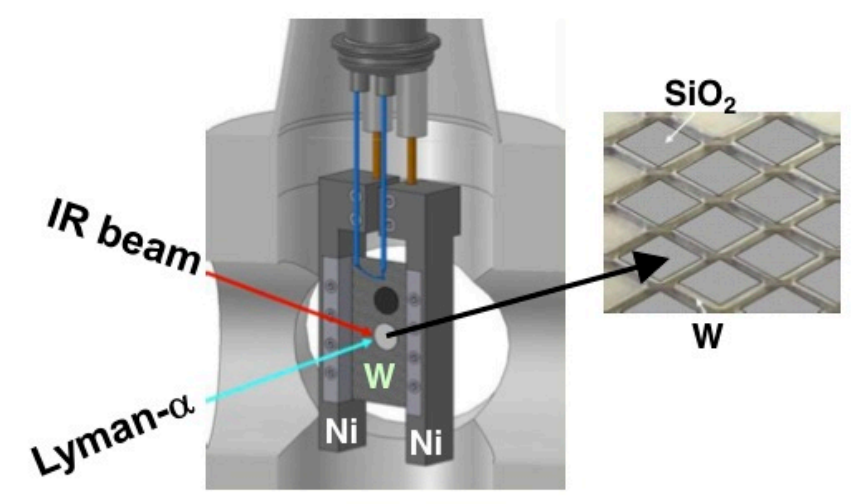

Figure 6.3: $\mathrm{SiO}_{2}$ sample pressed into a tungsten grid stretched between two $\mathrm{Ni}$ clamps which are cooled by $I-N_{2}$. The grid is at $45^{\circ}$ to Lyman- $\alpha$ radiation and to the incident IR radiation.

\subsubsection{Control Experiments - Heating by Lyman- $\alpha$ Lamp}

We measured whether the Lyman- $\alpha$ lamp caused appreciable warming of the $\mathrm{SiO}_{2}$ sample leading to thermal desorption of adsorbed $\mathrm{N}_{2} \mathrm{O}$ by adjusting the emitted radiation output of an incandescent lamp placed at the $\mathrm{MgF}_{2}$ window to match that of the Lyman- $\alpha$ lamp, both measured by a thermopile at the $\mathrm{SiO}_{2}$ sample position. Careful measurements by IR spectroscopy of the rate of adsorbed $\mathrm{N}_{2} \mathrm{O}$ loss due to incandescent lamp heating at $71 \mathrm{~K}$ were made. These measurements showed that the rate of thermal desorption caused by the Lyman$\alpha$ lamp was less than $25 \%$ the rate of adsorbed $\mathrm{N}_{2} \mathrm{O}$ loss caused by Lyman- $\alpha$ radiation. Compared to the systematic errors present in this experiment, this possible effect may be neglected. 


\subsubsection{Gas Phase $\mathrm{N}_{2} \mathrm{O}$ Photochemistry}

For measurements of the gas phase $\mathrm{N}_{2} \mathrm{O}$ photolysis, the $\mathrm{SiO}_{2}$ sample is removed from the cell. The chamber is filled with $\mathrm{N}_{2} \mathrm{O}$ gas at room temperature to a pressure of 10 Torr. At this pressure, a gas mixing time of $34 \mathrm{~s}$ along the longest dimension of the cell is estimated, and because spectral acquisition times of $600 \mathrm{~s}$ were employed it was assumed that complete mixing occurs. The gas volume sampled by the convergent IR beam is $12.5 \mathrm{~cm}^{3}$.

\subsection{5 $\mathrm{N}_{2} \mathrm{O}$ Gas Adsorption}

Gas adsorption is carried out by dosing $\mathrm{N}_{2} \mathrm{O}$ gas over periods of several minutes from a gas dosing tube of $0.6 \mathrm{~cm}$ diameter which faces the front side of the $\mathrm{SiO}_{2}$ sample at $71 \mathrm{~K}$. Diffusion of $\mathrm{N}_{2} \mathrm{O}$ into the porous sample is achieved by briefly warming to $90 \mathrm{~K}$ after dosing. The absence of $\mathrm{N}_{2} \mathrm{O}$ ice in these experiments was assured by the observation that small characteristic ice bands of $\mathrm{N}_{2} \mathrm{O}$ were removed by this annealing procedure.

The $\mathrm{SiO}_{2}$ sample used in this work has been briefly heated to $1000 \mathrm{~K}$ in vacuum prior to $\mathrm{N}_{2} \mathrm{O}$ adsorption and contained only isolated $\mathrm{Si}-\mathrm{OH}$ groups exhibiting a sharp $\mathrm{O}-\mathrm{H}$ stretching mode at $3751 \mathrm{~cm}^{-1}$ as shown in Figure 6.4. Adsorbed water is found absent in the IR spectrum. The density of the $\mathrm{Si}-\mathrm{OH}$ groups has been measured to be $2.8 \mathrm{Si}-\mathrm{OH} \mathrm{nm}{ }^{-2} ; 26$ hence the $2.2 \times 10^{-3} \mathrm{~g}$ sample (4400 $\mathrm{cm}^{2}$ surface area) contains $\sim 1 \times 10^{18} \mathrm{Si}-\mathrm{OH}$ groups. Figure 6.4 shows the spectrum of the isolated $\mathrm{Si}-\mathrm{OH}$ groups, and the conversion of these groups to associated $\mathrm{Si}-\mathrm{OH}$ groups as $\mathrm{N}_{2} \mathrm{O}$ is adsorbed using several doses at $90 \mathrm{~K}$. By adding $\mathrm{N}_{2} \mathrm{O}$ to the sample until 0.65 of the isolated $\mathrm{Si}-\mathrm{OH}$ groups have been converted to associated $\mathrm{Si}-\mathrm{OH} \cdots \mathrm{ON}_{2}$ species, as judged by a $65 \%$ decrease in integrated absorbance of the isolated $\mathrm{Si}-\mathrm{OH}$ groups, we adjust the starting number of adsorbed $\mathrm{N}_{2} \mathrm{O}$ species to $6.5 \times 10^{17}$ in our photochemical decomposition experiments. The initial coverage of $\mathrm{Si}-\mathrm{OH} \cdots \mathrm{ON}_{2}$ species in our experiments is therefore about $1.5 \times 10^{14} \mathrm{~N}_{2} \mathrm{O} \mathrm{cm}$, which is equivalent to about 
0.3 monolayers of adsorbed $\mathrm{N}_{2} \mathrm{O}$ on the $\mathrm{SiO}_{2}$ surface.

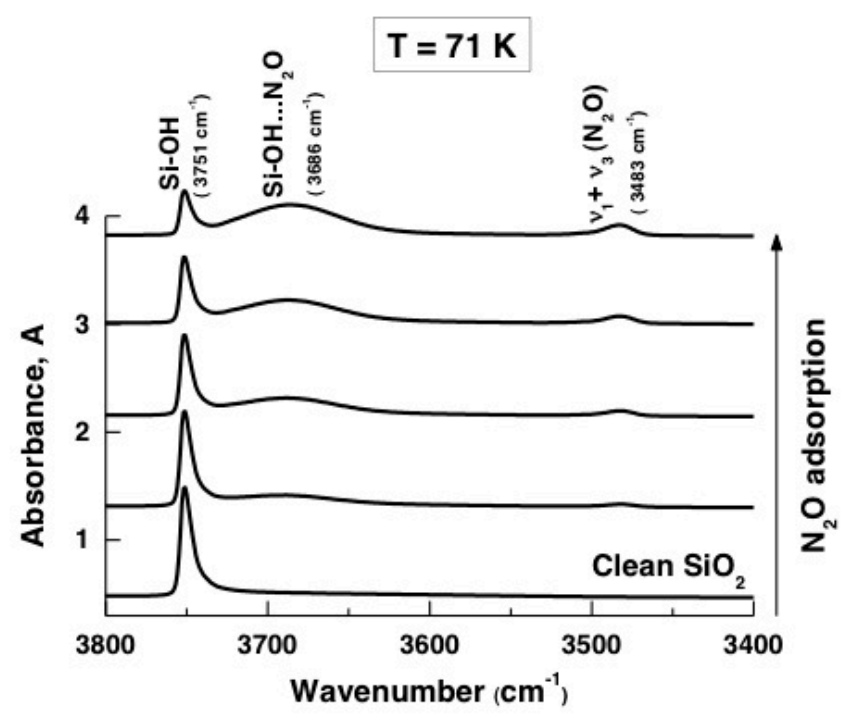

Figure 6.4: Evolution of associated $\mathrm{Si}-\mathrm{OH} \cdots \mathrm{ON}_{2}$ bands in the IR spectra during $\mathrm{N}_{2} \mathrm{O}$ adsorption on clean $\mathrm{Si}-\mathrm{OH}$.

It is likely that $\mathrm{Si}-\mathrm{OH}$ anchor groups provide relatively homogeneous adsorption sites for $\mathrm{N}_{2} \mathrm{O}$ compared to the inhomogeneous sites characteristic of most non- hydroxylated oxide surfaces. It is well known that other adsorbed molecules such as $\mathrm{CO}$ and $\mathrm{N}_{2}$ also preferentially bond to $\mathrm{SiO}_{2}$ surfaces by hydrogen bonding to $\mathrm{Si}-\mathrm{OH}$ groups, creating associated hydroxyl species at low temperatures[28], as found here for $\mathrm{N}_{2} \mathrm{O}$. Other mineral oxides containing surface $-\mathrm{OH}$ groups also exhibit the same selective adsorption on $\mathrm{OH}$ groups at cryogenic temperatures due to the polar nature of these groups[29, 30]. The hydrogen-bonding of small molecules to $\mathrm{OH}$ groups on the surface of water ices found on dust grains in the interstellar medium may also involve similar hydrogen bonding as found for $\mathrm{Si}-\mathrm{OH}$ groups on $\mathrm{SiO}_{2}$ surfaces studied here. And indeed for silicate interstellar grains, $\mathrm{Si}-\mathrm{OH}$ adsorption sites may also be present. 


\subsubsection{IR Spectral Measurements}

Transmission IR spectra through the $\mathrm{SiO}_{2}$ sample were measured using a purged Bruker Tensor 27 FTIR spectrometer. An external optical bench, also purged with dry- and $\mathrm{CO}_{2}$-free air, was used. Here IR radiation is focused on the $\mathrm{SiO}_{2}$ sample or for gas phase photochemical studies through the IR cell containing $\mathrm{N}_{2} \mathrm{O}(\mathrm{g})$. A I- $\mathrm{N}_{2}$ cooled $\mathrm{HgCdTe}$ detector was employed. Five hundred interferograms were averaged for each spectrum at $2 \mathrm{~cm}^{-1}$ resolution. The integrated absorbance, $\tilde{A}=\int A d n$, was measured for both gas phase species and adsorbed species in the various experiments, where $A=$ absorbance and $n=$ wavenumber. The background spectrum of the $\mathrm{SiO}_{2}$ sample was subtracted where appropriate. Three methods of integration of the infrared bands have been employed all yielding the same result within $\pm 5 \%$. The gas phase $\mathrm{N}_{2} \mathrm{O}$ calibrations of the integrated absorbance (ordinate) versus pressure yielded smooth linear curves, slightly convex downward at pressures below $\sim 0.8$ Torr (not shown). It is assumed in this work that the integrated absorbance measured for a particular vibrational mode is equally proportional to the number of molecules sampled by the IR beam in both the broadened spectrum in the gas phase and on the surface[31, 32].

An example of the Lyman- $\alpha$ induced decrease in $\int A d n$ for $\mathrm{N}_{2} \mathrm{O}(\mathrm{g})$ at 10 Torr pressure and $300 \mathrm{~K}$ is given in Figure 6.5, monitoring both the $2 v_{1}$ and the $\left(v_{1}+\right.$ $v_{3}$ ) modes. Here only $13 \%$ of the $\mathrm{N}_{2} \mathrm{O}(\mathrm{g})$ has been consumed in the experiment, and we can therefore consider the linear rate measured to be the initial rate. 


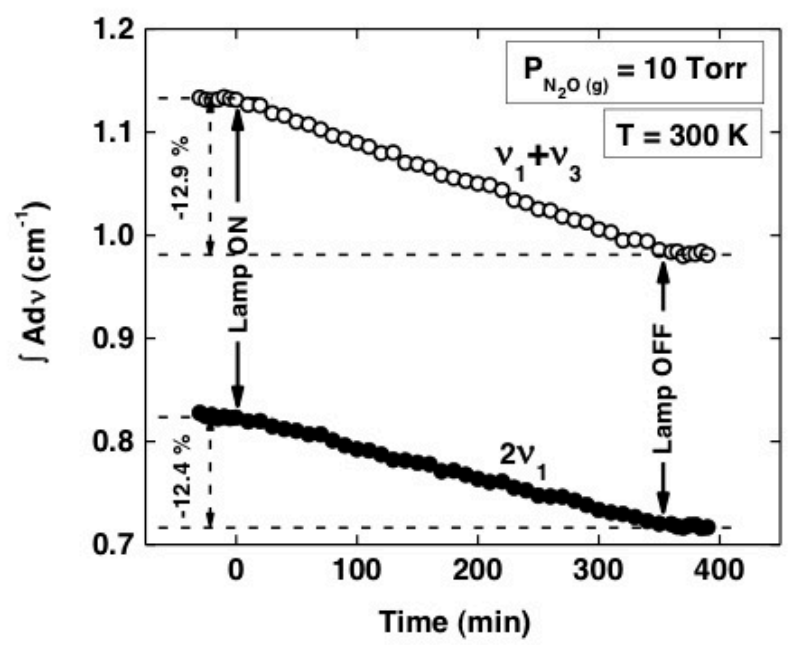

Figure 6.5: Lyman- $\alpha$ induced decrease in integrated absorbance for $\mathrm{N}_{2} \mathrm{O}(\mathrm{g})$ (10 Torr, 300K) modes $v_{1}+v_{3}$ and $2 v_{1}$. Here only $13 \%$ of $\mathrm{N}_{2} \mathrm{O}(\mathrm{g})$ has been consumed in the experiment.

\subsubsection{The Availability of Surface Sites for Photochemistry - Comparison of Gas Phase to Adsorbed Phase Measurements}

Our measurements of $\mathrm{N}_{2} \mathrm{O}$ photodecomposition in either the adsorbed layer or in the gas phase at 10 Torr are made under conditions where all incident Lyman- $\alpha$ photons are absorbed at the front of the sample. For the gas phase measurements, at 10 Torr, total absorption occurs in the first $\mathrm{cm}$ of pathlength, just behind the $\mathrm{MgF}_{2}$ window. It is likely that some surface recombination events occur on the $\mathrm{MgF}_{2}$ window producing a small yield of combination product molecules which are observed by IR. The geometrical availability of the $\mathrm{MgF}_{2}$ surface for recombination events is extremely small compared to the interior sites of the porous $\mathrm{SiO}_{2}$.

For the adsorbed phase we estimate that complete absorption or scattering of 
Lyman- $\alpha$ radiation occurs in the outer $1 / 5$ of the $\mathrm{SiO}_{2}$ sample depth. The estimate of the depth of activity of the Lyman- $\alpha$ radiation in the $\mathrm{SiO}_{2}$ is based on the observation that the photochemical depletion of adsorbed $\mathrm{N}_{2} \mathrm{O}$ ceases after about $20 \%$ depletion. Since the absorption length for Lyman- $\alpha$ radiation in $\mathrm{SiO}_{2}$ is about $10-100 \mathrm{~nm}$, the larger penetration depth observed here $\left(1 / 5\right.$ of $\mathrm{SiO}_{2}$ sample thickness $\approx 10^{4} \mathrm{~nm}$ ) must be caused by extensive light scattering in the powered $\mathrm{SiO}_{2}$.

\subsection{Results}

\subsubsection{Photo-stability of Si-OH Groups by Lyman- $\alpha$ Radiation}

To be certain that the photo-depletion of adsorbed $\mathrm{N}_{2} \mathrm{O}$ was not related to radiation damage of $\mathrm{Si}-\mathrm{OH}$ groups, a photodecomposition experiment using Lyman- $\alpha$ radiation was performed on the bare isolated $\mathrm{Si}-\mathrm{OH}$ groups. The results obtained over a 600 minute exposure are shown in Figure 6.6. Using the integrated $-\mathrm{OH}$ absorbance versus time, in which a barely detectable loss of $\mathrm{Si}$ $\mathrm{OH}$ is observed at $300 \mathrm{~K}$, we estimate that the cross section for Lyman- $\alpha$ photolysis of $\mathrm{Si}-\mathrm{OH}$ groups held in the geometry of this experiment is $\mathrm{Q}_{\mathrm{Si}-\mathrm{OH}} \approx$ $3.1 \times 10^{-21} \mathrm{~cm}^{2}$ assuming the outer $1 / 5$ of the $\mathrm{SiO}_{2}$ surface (penetration depth $=10^{4}$ $\mathrm{nm}$ ) receives Lyman- $\alpha$ radiation. This average $\mathrm{Si}-\mathrm{OH}$ depletion cross section is therefore negligible in our experiments compared to the cross section for $\mathrm{N}_{2} \mathrm{O}$ depletion, as shown in Table 6.1. The destruction of Si-OH groups by Lyman- $\alpha$ irradiation may therefore be neglected in the experiment with adsorbed $\mathrm{N}_{2} \mathrm{O}$. 


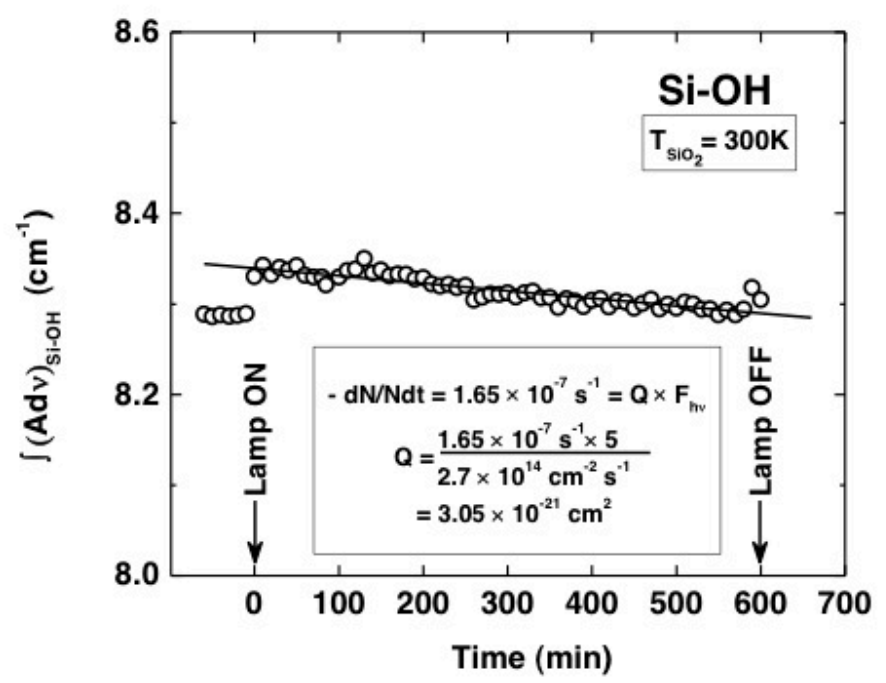

Figure 6.6: Lyman- $\alpha$ photolysis of clean $\mathrm{SiO}_{2}$ containing only isolated $\mathrm{Si}-\mathrm{OH}$ groups. The factor of 5 in the calculation is due to the approximation that only the outer $1 / 5$ of the $\mathrm{SiO}_{2}$ surfaces receives Lyman- $\alpha$ radiation due to strong absorption and scattering.

\subsubsection{Photodesorption of $\mathrm{N}_{2} \mathrm{O}$ from $\mathrm{Si}-\mathrm{OH}$ Sites}

Using a quadrupole mass spectrometer in the vacuum system, we found that photodesorption of $\mathrm{N}_{2} \mathrm{O}$ from $\mathrm{N}_{2} \mathrm{O}$ ice condensed on the sample support assembly at $71 \mathrm{~K}$, dominates when the shutter is opened due to receipt of scattered Lyman- $\alpha$ radiation inside the cell. Therefore an estimate of the cross section for photodesorption of $\mathrm{N}_{2} \mathrm{O}$ from the $\mathrm{SiO}_{2}$ cannot be made. Because most of the adsorbed $\mathrm{N}_{2} \mathrm{O}$ is contained deep in the porous $\mathrm{SiO}_{2}$, it is unlikely that extensive photodesorption will occur to deplete adsorbed $\mathrm{N}_{2} \mathrm{O}$, since readsorption will readily occur along the pore walls at $71 \mathrm{~K}$.

\subsubsection{Qualitative Comparison of Gas Phase and Surface Phase Photo- decomposition of $\mathrm{N}_{2} \mathrm{O}$}

Figure 6.7 shows a qualitative comparison of the photo-decomposition of $\mathrm{N}_{2} \mathrm{O}$ 
in the gas phase to $\mathrm{N}_{2} \mathrm{O}$ photo-decomposition on the $\mathrm{SiO}_{2}$ surface. The left hand panel shows the loss of $\mathrm{N}_{2} \mathrm{O}(\mathrm{g})$ through the observation of the decrease in absorbance of four prominent overtone or combination IR bands for the molecule. Unresolved vibration-rotation spectra are recorded. Over 6 hours of irradiation, the $\mathrm{NO}(\mathrm{g})$ stretching mode $\left(1876 \mathrm{~cm}^{-1}\right)$ and an $\mathrm{NO}_{2}(\mathrm{~g})$ stretching mode at 1617 $\mathrm{cm}^{-1}$ are observed to intensify as photo-decomposition followed by fragment reaction take place in the gas phase. For comparison, the same spectral regions are shown in the right-hand panel for adsorbed $\mathrm{N}_{2} \mathrm{O}$ on $\mathrm{SiO}_{2}$ subjected to Lyman$\alpha$ irradiation. It may be seen that for the $\mathrm{N}_{2} \mathrm{O}_{4}$ species produced by fragment combination, a large yield is observed on the $\mathrm{SiO}_{2}$ surface, while the gas phase measurements barely detect this molecule with a stretching frequency near 1739 $\mathrm{cm}^{-1}$. This observation qualitatively indicates that fragment combination effects to produce more complex molecules are favored in the adsorbed layer.
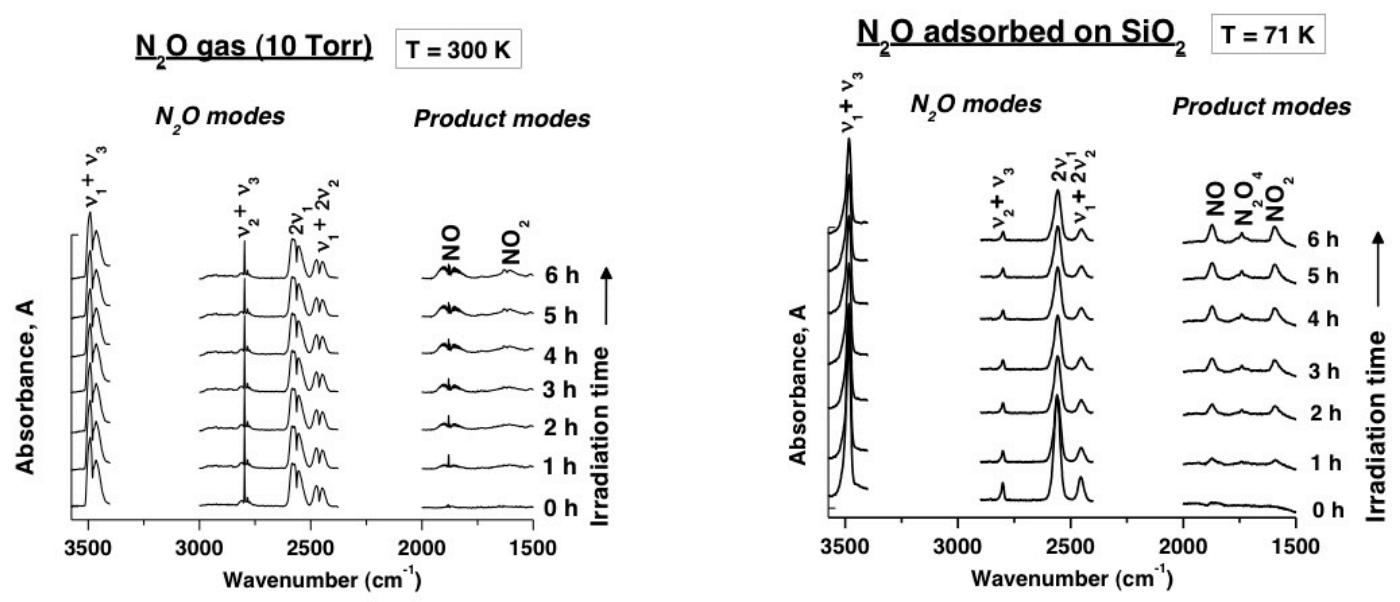

Figure 6.7: A qualitative comparison of the infrared spectral evolution during the photodecomposition of $\mathrm{N}_{2} \mathrm{O}$ in the gas phase $(300 \mathrm{~K})$ and in the adsorbed phase $(71 \mathrm{~K})$. $\mathrm{N}_{2} \mathrm{O}_{4}$ species produced by the fragment combination are observed on the $\mathrm{SiO}_{2}$ surface while the gas phase measurements barely detect this species. 


\subsubsection{Quantitative Measurement of the Photochemical Rates by Infrared Spectroscopy}

Figure 6.8 shows an example of quantitative measurements of the depletion of $\mathrm{N}_{2} \mathrm{O}$ on the $\mathrm{SiO}_{2}$ surface when irradiated, using various absorption bands at 71 $\mathrm{K}$ where the fractional $\mathrm{N}_{2} \mathrm{O}$ coverage is plotted. In these experiments, the shutter is alternatively opened and closed over a total experimental period of about 840 min. as loss of $\mathrm{N}_{2} \mathrm{O}$ absorbance occurs by two processes. When the shutter is closed, a slow thermal desorption process is observed at $71 \mathrm{~K}$, yielding a slope in integrated absorbance of $m_{0}$ for the initial segment (green line) just before the shutter is opened. When the shutter is opened, a steeper initial slope, $m_{1}$, is observed in the second segment (red line) of the experiment. This slope is the result of combined thermal desorption and photo-depletion of $\mathrm{N}_{2} \mathrm{O}$. The difference between the slopes, $\left[m_{1}-m_{0}\right]$, is a measure of the photo-depletion kinetics of $\mathrm{N}_{2} \mathrm{O}$, corrected for thermal desorption as measured from the slope $m_{0}$. In the experimental sequence shown, three separate $\mathrm{N}_{2} \mathrm{O}$ photo-depletion rates are measured as labeled Photodissociation I, II and III. As expected, these rates systematically decrease as $\mathrm{N}_{2} \mathrm{O}$ is lost from the outer depletion layer region of the $\mathrm{SiO}_{2}$ powder. 

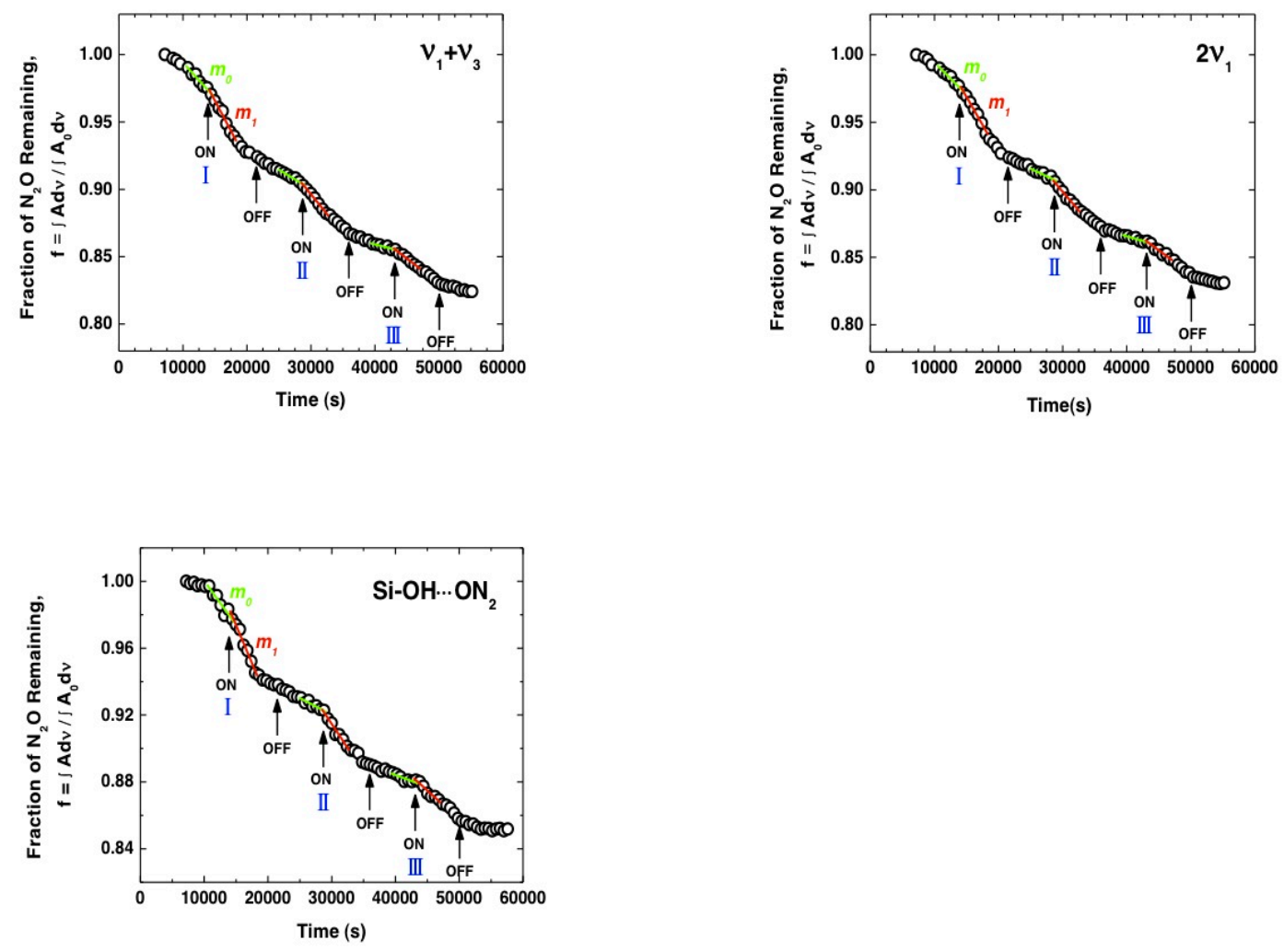

Figure 6.8: An example of quantitative measurements of the depletion of $\mathrm{N}_{2} \mathrm{O}$ on the $\mathrm{SiO}_{2}$ surface at $71 \mathrm{~K}$ during Lyman- $\alpha$ irradiation in three sequential experiments, I, II and III. The shutter is alternatively opened and closed thereby turning the light on and off. Loss of absorbance occurs in vacuum by two processes: thermal \& thermal + photodesorption of $\mathrm{N}_{2} \mathrm{O}$ (slopes $\mathrm{m}_{0}$ and $\mathrm{m}_{1}$ ).

The photodepletion of adsorbed $\mathrm{N}_{2} \mathrm{O}$ from $\mathrm{Si}-\mathrm{OH} \cdots \mathrm{ON}_{2}$ adsorbate species was also monitored by the measurements of the conversion of the broad Si$\mathrm{OH} \cdots \mathrm{ON}_{2}$ band to the uncomplexed isolated $\mathrm{Si}-\mathrm{OH}$ band. Figure 6.8 shows that as $\mathrm{N}_{2} \mathrm{O}$ is consumed photochemically, the $\mathrm{Si}-\mathrm{OH} \cdots \mathrm{ON}_{2}$ complex also decreases in coverage. Measurements of the fractional decrease in Si-OH$\cdots \mathrm{ON}_{2}$ coverage during the irradiation period in experiments I, II and III correlate well within $\pm 10 \%$ with measurements of the fractional decrease in $\mathrm{N}_{2} \mathrm{O}$ coverage, as based on measurements of absorbance changes for the adsorbed $\mathrm{N}_{2} \mathrm{O}$ molecules. 
Table 6.1 presents the data for the three sequential $\mathrm{N}_{2} \mathrm{O}$ photodecomposition experiments, I, II, and III.

\begin{tabular}{|c|c|c|c|}
\hline \multirow[t]{2}{*}{$\mathrm{N}_{2} \mathrm{O}$ mode } & \multicolumn{3}{|c|}{$-\mathrm{dN} / \mathrm{Ndt}=\Delta$ (fractional $\mathrm{N}_{2} \mathrm{O}$ change in total $\mathrm{SiO}_{2}$ volume) $\left(\mathrm{s}^{-1}\right)$} \\
\hline & Photodissociation I & Photodissociation II & Photodissociation III \\
\hline$v_{1}+v_{3}$ & $3.71 \times 10^{-6}$ & $3.23 \times 10^{-6}$ & $2.75 \times 10^{-6}$ \\
\hline $2 v_{1}$ & $3.53 \times 10^{-6}$ & $3.55 \times 10^{-6}$ & $2.41 \times 10^{-6}$ \\
\hline $\mathrm{SiOH} \cdots \mathrm{ON}_{2}$ & $3.85 \times 10^{-6}$ & $3.97 \times 10^{-6}$ & $2.35 \times 10^{-6}$ \\
\hline Average & $3.70 \times 10^{-6}$ & & \\
\hline \multirow[t]{2}{*}{$\mathrm{N}_{2} \mathrm{O}$ mode } & \multicolumn{3}{|c|}{ Cross Section for $\mathrm{N}_{2} \mathrm{O}$ depletion in outer $1 / 5$ of sample, $Q=5 \Delta / F_{h v}\left(\mathrm{~cm}^{2}\right)$} \\
\hline & Photodissociation I & Photodissociation II & Photodissociation III \\
\hline$v_{1}+v_{3}$ & $6.88 \times 10^{-20}$ & $5.98 \times 10^{-20}$ & $5.10 \times 10^{-20}$ \\
\hline $2 v_{1}$ & $6.53 \times 10^{-20}$ & $6.58 \times 10^{-20}$ & $4.47 \times 10^{-20}$ \\
\hline $\mathrm{SiOH} \cdots \mathrm{ON}_{2}$ & $7.13 \times 10^{-20}$ & $7.36 \times 10^{-20}$ & $4.35 \times 10^{-20}$ \\
\hline Average & $6.85 \times 10^{-20}$ & & \\
\hline \multirow[t]{2}{*}{$\mathrm{N}_{2} \mathrm{O}$ mode } & \multicolumn{3}{|c|}{ Quantum Yield, Q.Y. $\left(N_{2} O\right.$ molecules/photon $)=-[d N / d t] / F_{h v} A=-(\Delta \cdot N) / F_{h v} A$} \\
\hline & Photodissociation I & Photodissociation II & Photodissociation III \\
\hline$v_{1}+v_{3}$ & 0.006 & 0.005 & 0.004 \\
\hline $2 v_{1}$ & 0.006 & 0.006 & 0.004 \\
\hline $\mathrm{SiOH} \cdots \mathrm{ON}_{2}$ & 0.006 & 0.006 & 0.004 \\
\hline Average & 0.005 & & \\
\hline
\end{tabular}

In Table 6.1, the cross sections measured for $\mathrm{N}_{2} \mathrm{O}$ depletion by Lyman- $\alpha$ irradiation are shown for photodissociation experiments I, II, and III. These cross sections are averages calculated for depletion of $\mathrm{N}_{2} \mathrm{O}$ in the outer $1 / 5$ of the $\mathrm{SiO}_{2}$ thickness and the fractional change along each photodissociation segment is designated $\Delta$. A photodissociation cross section of $6.85 \times 10^{-20} \mathrm{~cm}^{2}$ is measured averaging over the three measurements made in Experiment I. Also, the quantum yields (Q.Y.) for the three sequential experiments are shown. An average initial Q.Y. for Experiment I of order 0.005 is also measured over the depletion depth.

The decrease in cross section and Q.Y. for sequential experiments I, II, and III may be simply understood by the enhanced effect of $\mathrm{SiO}_{2}$ absorption and 
scattering of Lyman- $\alpha$ radiation as the depth of remaining $\mathrm{N}_{2} \mathrm{O}$ increases in the three experiments. In the later experiments, more and more Lyman- $\alpha$ attenuation due to the $\mathrm{SiO}_{2}$ occurs in the region containing the majority of adsorbed $\mathrm{N}_{2} \mathrm{O}$.

Figure 6.9 shows an example of the production of $\mathrm{N}_{2} \mathrm{O}_{4}$ by photodecomposition of $\mathrm{N}_{2} \mathrm{O}$ adsorbed on $\mathrm{SiO}_{2}$. Here, in the sequential experiments (Photodissociation I, II and III) corresponding to those shown in Figure 6.8, three regions of photo- production of $\mathrm{N}_{2} \mathrm{O}_{4}$ may be discerned. The measured $\mathrm{N}_{2} \mathrm{O}_{4}$ formation rates, corresponding to the three decreasing rates of $\mathrm{N}_{2} \mathrm{O}$ photo-depletion in Figure 6.8, are observed to diminish as the experiment continues through three full cycles. Similar observations about $\mathrm{NO}$ and $\mathrm{NO}_{2}$ production have also been made.

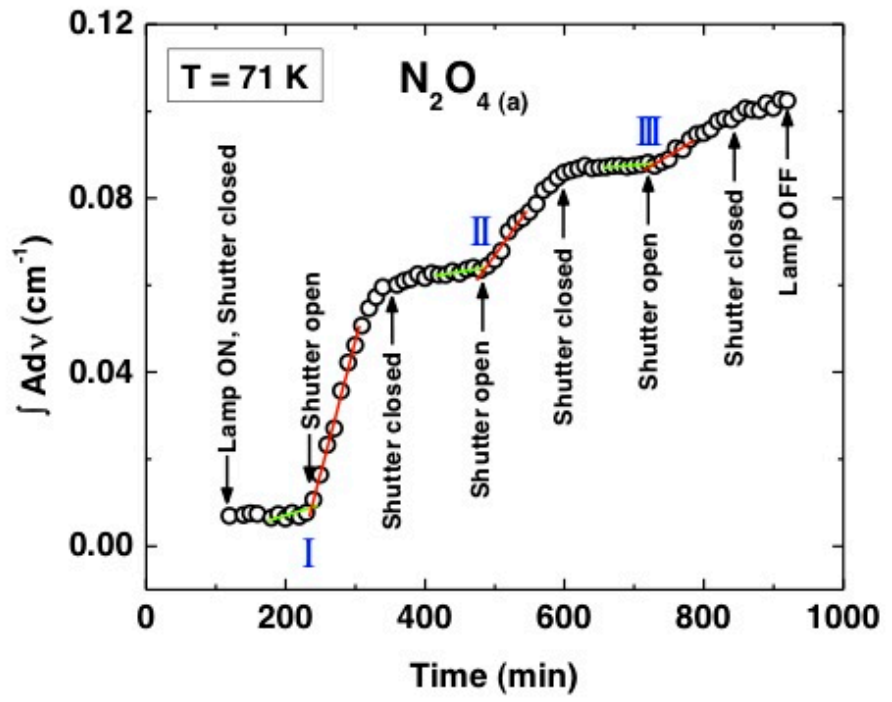

Figure 6.9: An example of the production of $\mathrm{N}_{2} \mathrm{O}_{4}$, one of the combination products of photodecomposition of $\mathrm{N}_{2} \mathrm{O}$ adsorbed on $\mathrm{SiO}_{2}$.

We assume that Beer's law and the IR integrated absorption coefficient, $\varepsilon$, apply for both the gas phase species and the adsorbed species for a particular 
vibrational mode. Measuring the integrated absorbance, $\tilde{A}=\int A d n$, we may write

$$
\mathrm{d} \tilde{\mathrm{A}} / \mathrm{dt}=\varepsilon \mathrm{d}(C D) / \mathrm{dt}
$$

where $(C D)=$ the column density (molecules $\mathrm{cm}^{-2}$ ) and $\varepsilon=\mathrm{IR}$ integrated absorption coefficient. For our studies, the ratio of the rate of production of a product to the rate of consumption of $\mathrm{N}_{2} \mathrm{O}$ has been measured. Thus for the gas phase we may write that the ratio of the rate of production of a product to the rate of consumption of $\mathrm{N}_{2} \mathrm{O}$ reactant is

$$
\left[\frac{1}{\varepsilon} d \tilde{A} / d t\right]_{\text {product }} /\left[\frac{1}{\varepsilon} d \tilde{A} / d t\right]_{\text {product }}=R_{g}
$$

$\mathrm{R}_{\mathrm{g}}$ will involve the ratio of integrated absorption coefficients, $\varepsilon_{\mathrm{N} 2 \mathrm{O}} / \varepsilon_{\text {product }}$. Likewise a ratio $R_{a}$ for the adsorbed species may be measured giving the ratio of the rate of production of a combination product in the adsorbed layer to the rate of $\mathrm{N}_{2} \mathrm{O}(\mathrm{a})$ consumption. These ratios, $R_{g}$ and $R_{a}$, both contain integrated $I R$ absorption coefficients of the product molecule and the $\mathrm{N}_{2} \mathrm{O}$ (either in the gas phase or in the absorbed phase). We assume that the individual absorption coefficients, $\varepsilon$, of $\mathrm{N}_{2} \mathrm{O}, \mathrm{NO}, \mathrm{NO}_{2}$ and $\mathrm{N}_{2} \mathrm{O}_{4}$ in the gas and in the adsorbed phases are closely similar for each molecule, resulting in a cancellation or near cancellation in the ratio $R_{a} / R_{g}=S_{a}$ [33]. The ratio $R_{a} / R_{g}=S_{a}$ is a measure of the enhancement factor or selectivity factor for the rate of a combination reaction on the $\mathrm{SiO}_{2}$ surface compared to that in the gas phase. Table 6.2 shows values of $S_{a}$ and $\bar{S}_{a}$ (averaged from two vibrational modes). Systematic behavior of $\bar{S}_{a}$ is evident: [ $\left.\bar{S}_{a}\left(\mathrm{~N}_{2} \mathrm{O}_{4}\right) \approx 20\right]>\left[\bar{S}_{a}\left(\mathrm{NO}_{2}\right) \approx 5\right]>\left[\bar{S}_{a}(\mathrm{NO}) \approx 1\right]$ for experiment I. Photodissociation II and III are not employed in our analysis for reasons to be discussed later. These values of $\bar{S}_{a}$ for the three photoproduct molecules, along with the error bar in the average over the three measurements of $\mathrm{N}_{2} \mathrm{O}$ depletion using three IR modes, are shown in Figure 6.10 [34]. The values of $\bar{S}_{a}$ measured here are independent of the attenuation of Lyman- $\alpha$ radiation by the $\mathrm{SiO}_{2}$ since $R_{a}$ and $R_{g}$ 
are each ratios of rates which are linearly dependent on light intensity. In other words, using the rate of loss of $\mathrm{N}_{2} \mathrm{O}$ in the gas and on the surface as a normalization factor leads to an independence of both $\mathrm{R}_{\mathrm{a}}$ and $\mathrm{R}_{\mathrm{g}}$ and $\bar{S}_{a}$ on light intensity.

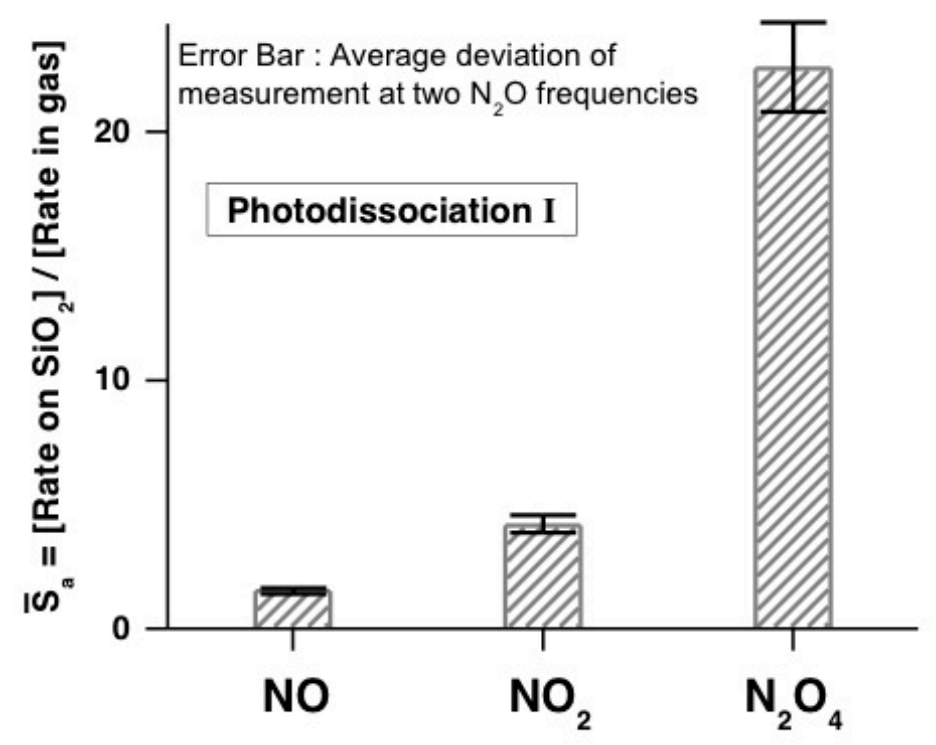

Figure 6.10: Variation of average complex product enhancement ratio, $\bar{S}_{a}$ with the complexity of the combination products.

\subsubsection{Quantum Efficiency of $\mathrm{N}_{2} \mathrm{O}$ Photodecomposition on High $\mathrm{Area}^{\mathrm{SiO}_{2}}$}

From Table 6.1 the quantum yield for $\mathrm{N}_{2} \mathrm{O}$ photodecomposition has been measured in three experiments involving $\left(2 v_{1}\right),\left(v_{1}+v_{3}\right)$, and $n\left(\mathrm{Si}-\mathrm{OH}^{\circ} \cdots \mathrm{ON}_{2}\right)$ vibrational modes. An average photodecomposition cross section based on Experiment I of $6.85 \times 10^{-20} \mathrm{~cm}^{2}$ is measured. Because of the low probability of photodesorption from the pore structure of the $\mathrm{SiO}_{2}$, we believe this measured cross section is closely similar to the photodissociation cross section for the geometry studied here. This low magnitude of the cross section for the adsorbed 
$\mathrm{N}_{2} \mathrm{O}$ suggests that strong absorption by, and possibly light scattering from the powdered $\mathrm{SiO}_{2}$ substrate, remove a large fraction of the Lyman- $\alpha$ radiation. The measured quantum yield on the $\mathrm{SiO}_{2}($ Q.Y. 0.005) differs significantly from that in the gas phase (Q.Y. $\left(\mathrm{N}_{2} \mathrm{O}(\mathrm{g})\right)=1.46 \pm 0.04 \mathrm{~N}_{2} \mathrm{O}$ /photon). [35] These results are consistent with the high absorption coefficient and scattering ability of the $\mathrm{SiO}_{2}$ particles for Lyman- $\alpha$ radiation[36] which removes much of the incident light. In

addition quenching of excited $\mathrm{N}_{2} \mathrm{O}$ species or fragments by contact with $\mathrm{SiO}_{2}$ may be responsible for part of the decrease in Q.Y.

\subsection{Discussion}

\subsubsection{Efficiency of Complex Molecule Formation from Lyman-a Photo- decomposition of $\mathrm{N}_{2} \mathrm{O}$}

The measurements reported here allow us to estimate the overall selectivity of certain secondary reactions from $\mathrm{N}_{2} \mathrm{O}$ photodecomposition resulting in combination reactions to produce products such as $\mathrm{NO}, \mathrm{NO}_{2}$ and $\mathrm{N}_{2} \mathrm{O}_{4}$. $A$ comparison is made of the relative rate, $\mathrm{R}$, of combination-product production to the rate of $\mathrm{N}_{2} \mathrm{O}$ consumption, either on the $\mathrm{SiO}_{2}$ surface $\left(\mathrm{R}_{\mathrm{a}}\right)$ or in the gas phase at 10 Torr pressure $\left(R_{g}\right)$. At the $\mathrm{N}_{2} \mathrm{O}$ coverage used here $(\sim 0.3 \mathrm{ML})$, the transit distance for a photofragment from a $\mathrm{N}_{2} \mathrm{O}$ (a) photodecomposition event to a neighboring adsorbed $\mathrm{N}_{2} \mathrm{O}$ (a) molecule is a few nanometers at most, since most of the adsorbate molecules exist in close proximity on the interior surface of internal pores of the $\mathrm{SiO}_{2}$. The depth of the photo-depletion region at the front of the sample of $\mathrm{SiO}_{2}$ is of order $10^{4} \mathrm{~nm}$, meaning that escape of either an $\mathrm{N}_{2} \mathrm{O}$ molecule or a molecular fragment to vacuum is very unlikely from a single photoexcitation event. In comparison, at 10 Torr pressure, the mean free path of an $\mathrm{N}_{2} \mathrm{O}$ molecule is $2.6 \times 10^{4} \mathrm{~nm}$. The rate of a termolecular reaction of a radical species with $\mathrm{N}_{2} \mathrm{O}$ is vanishingly small at 10 Torr compared to the bimolecular reactions observed, causing the gas phase process to be a good control 
involving essentially zero third body reactions. Fragments from the photodecomposition of $\mathrm{N}_{2} \mathrm{O}$ in the gas phase experiments will experience a similar mean free path as do $\mathrm{N}_{2} \mathrm{O}$ molecules in the gas. Thus, on both the surface and in the gas phase, collisions of photofragments and neighboring undissociated $\mathrm{N}_{2} \mathrm{O}$ molecules will occur predominantly for all fragments over a distance range from a few $\mathrm{nm}$ (surface) to $\sim 10^{4} \mathrm{~nm}$ (gas). Assuming rapid freeradical type reactivity for the fragment plus an $\mathrm{N}_{2} \mathrm{O}$ molecule, escape of the fragment from a single photoexcitation event from the $\mathrm{SiO}_{2}$ pores will be unlikely. Thus the loss of $\mathrm{N}_{2} \mathrm{O}$ can be attributed to its photodissociation combined with $\mathrm{N}_{2} \mathrm{O}$ reaction with photofragments of $\mathrm{N}_{2} \mathrm{O}$. Under these conditions of complete reaction of a fragment with $\mathrm{N}_{2} \mathrm{O}$, the average value $\bar{S}_{a}$ will then be a measure of the selectivity compared to the gas phase of a surface combination reaction beginning with a [fragment] $\cdots\left[\mathrm{N}_{2} \mathrm{O}\right]$ collision (ultimately producing a specific secondary product molecule). Values of the average selectivity factor $\bar{S}_{a}>1$ will indicate that the surface combination processes are favored.

Table 6.2 shows the values of the selectivity factors for surface combination reactions for several products of $\mathrm{N}_{2} \mathrm{O}$ photodecomposition. Values of $\mathrm{S}_{a}$ and $\bar{S}_{a}$ are shown for three combination products $\mathrm{NO}, \mathrm{NO}_{2}$ and $\mathrm{N}_{2} \mathrm{O}_{4}$. The values of $\mathrm{S}_{a}$ are measured for the three combination products using the two vibrational modes $\left(v_{1}+v_{3}\right)$ and $\left(2 v_{1}\right)$ for the measurement of the $\mathrm{N}_{2} \mathrm{O}$ photo-depletion rate. 
Table 6.2 Selectivity of Combination Processes

\begin{tabular}{|l|l|l|l|l|}
\hline \multirow{2}{*}{$\mathbf{N}_{\mathbf{2}} \mathbf{O}$ mode } & Photo-product & \multicolumn{3}{|c|}{$\mathbf{S}_{\mathbf{a}}=\mathbf{R}_{\mathbf{a}} / \mathbf{R}_{\mathbf{g}}$} \\
\cline { 3 - 5 } & & Photodissociation I & Photodissociation II & Photodissociation III \\
\hline \multirow{3}{*}{$v_{1}+v_{3}$} & $\mathrm{NO}$ & 1.76 & 0.73 & 0.64 \\
& $\mathrm{NO}_{2}$ & 4.86 & 2.68 & 1.26 \\
& $\mathrm{~N}_{2} \mathrm{O}_{4}$ & 26.15 & 12.57 & 3.54 \\
\hline \multirow{2}{*}{$2 v_{1}$} & $\mathrm{NO}$ & 1.27 & 0.40 & 0.52 \\
& $\mathrm{NO}_{2}$ & 3.44 & 1.49 & 1.41 \\
\hline Average from & $\mathrm{N}_{2} \mathrm{O}_{4}$ & 18.95 & 7.01 & 3.92 \\
two modes, $\overline{\mathrm{S}}_{\mathrm{a}}$ & $\mathrm{NO}$ & $1.51 \pm 0.24$ & $0.56 \pm 0.16$ & $0.58 \pm 0.06$ \\
& $\mathrm{NO}_{2}$ & $4.15 \pm 0.71$ & $2.08 \pm 0.59$ & $1.33 \pm 0.07$ \\
& $\mathrm{~N}_{2} \mathrm{O}_{4}$ & $22.55 \pm 3.60$ & $9.79 \pm 2.78$ & $3.73 \pm 0.19$ \\
\hline
\end{tabular}

It may be seen that the values of a systematically increase as the complexity of the photoproduct increases. Thus, as shown in Table 6.2 and Figure 6.10, the most complex photoproduct, $\mathrm{N}_{2} \mathrm{O}_{4}$, exhibits an average lower limit value of $\bar{S}_{a}=$ $22.5 \pm 3.6$, averaging over kinetic results obtained by the observation of two different vibrational modes of $\mathrm{N}_{2} \mathrm{O}$. Since $\mathrm{N}_{2} \mathrm{O}_{4}$ must be produced by several sequential radical combination reactions on the $\mathrm{SiO}_{2}$ surface, and if combination reactions are enhanced by the presence of the $\mathrm{SiO}_{2}$ surface, $\mathrm{N}_{2} \mathrm{O}_{4}$ should exhibit a large value of a compared to less complex products such as $\mathrm{NO}_{2}$ or $\mathrm{NO}$, as is observed. The relative rates of product formation shown in Figure 6.10 could also be influenced by photodecomposition of the products shown and is not addressed in this work. NO is also a primary photo-dissociation product (eqn. 2) as well as a radical combination product (eqn. 5), and would therefore be expected to exhibit the lowest value of $\bar{S}_{a}$, as is observed.

It should be mentioned that the enhanced combination rates on the $\mathrm{SiO}_{2}$ surface for $\mathrm{NO}_{2}$ and $\mathrm{N}_{2} \mathrm{O}_{4}$ will also be influenced by the lower temperature of the surface. Thus, Herbst has shown that unactivated three-body gas phase reactions will display in the range $100-300 \mathrm{~K} \mathrm{a}(1 / \mathrm{T})^{\mathrm{n}}$ power dependence where $\mathrm{n}$ is of order $1.3-3.5$ for different reactions and third bodies. This dependence is due to the involvement of different rotational partition functions for various three- 
body complexes and therefore to different rates of energy transfer to the third body at the time of bond formation[37]. The tendency for decreasing temperature to increase the rate of combination reactions is probably also involved in our measurements at $71 \mathrm{~K}$ for combination reactions on the $\mathrm{SiO}_{2}$ surface, acting as a third body.

\subsubsection{Decreasing Combination Reaction Rates as Photodecomposition Increases}

Table 6.2 reveals that for all three photoproducts, decreases systematically as the coverage of $\mathrm{N}_{2} \mathrm{O}$ decreases in the depletion layer in the top $1 / 5$ of the $\mathrm{SiO}_{2}$ powder layer where Lyman- $\alpha$ photons can reach adsorbed $\mathrm{N}_{2} \mathrm{O}$ molecules. Photodissociation segments I, II and III sample the photochemistry for decreasing coverages of $\mathrm{N}_{2} \mathrm{O}$ in the depletion region, accompanied by increasing photoproduct coverage. The reason for the systematic decrease in $\bar{S}_{a}$ for all three photoproduct molecules may be related to partial photodecomposition of product molecules as the $\mathrm{N}_{2} \mathrm{O}$ photodecomposition reaction proceeds, and as the coverage of product molecules increases in the depletion region. We therefore frame our discussion on photodissociation segment I where photodecomposition of products is minimized.

\section{The Role of the $\mathrm{SiO}_{2}$ Surface on the Rate of Combination Reactions}

Only for the $\mathrm{H}+\mathrm{H}$ recombination reaction has it been demonstrated experimentally that radical recombination (on olivine and graphite surfaces) is favored on the surface. The rate is essentially zero for this reaction in the gas phase in the interstellar medium because of the absence of three-body collisions needed to carry away the energy generated by the formation of the $\mathrm{H}-\mathrm{H}$ chemical bond. Our experiments demonstrate that for the model interstellar molecule, $\mathrm{N}_{2} \mathrm{O}$, surface combination reactions also contribute kinetic pathways leading to enhanced rates of complex nitrogen-oxide product formation. We believe that the 
ratios and trends observed in these experiments are best explained by the transfer of energy to the $\mathrm{SiO}_{2}$ substrate as a consequence of chemical bond formation processes as radical species exothermically convert into stable molecules. The pore structure of amorphous $\mathrm{SiO}_{2}$ enhances dramatically the role of surface combination processes. As more complex chemical species are observed by radio astronomy in the interstellar medium, it is likely that the role of surfaces in enhancing radical combination reactions (by transporting exothermicity away from combining molecules) will become a dominant model to explain the origin of complex molecules including those of importance in lifeforming processes.

\subsection{Summary of Results}

The following results have been obtained in this investigation:

1. It has been shown that the isolated hydroxyl groups on amorphous $\mathrm{SiO}_{2}$, present at a coverage of $\sim 2.8 \mathrm{Si}-\mathrm{OH} \mathrm{nm}{ }^{-2}$, act as hydrogen-bonding adsorption sites for $\mathrm{N}_{2} \mathrm{O}$ molecules at $71 \mathrm{~K}$. By using transmission IR spectroscopy, and observing the extent of conversion of the isolated groups to associated groups upon $\mathrm{N}_{2} \mathrm{O}$ adsorption, the coverage of hydroxyl-bound $\mathrm{N}_{2} \mathrm{O}$ molecules can be determined.

2. Lyman- $\alpha$ radiation, produced from a calibrated $\mathrm{Ar}-\mathrm{H}_{2}$ discharge lamp, has been used to compare the rate of the photo-decomposition of adsorbed $\mathrm{N}_{2} \mathrm{O}$ at a surface temperature of $71 \mathrm{~K}$ compared to $\mathrm{N}_{2} \mathrm{O}(\mathrm{g})$. Photoproducts such as $\mathrm{NO}$, $\mathrm{NO}_{2}$ and $\mathrm{N}_{2} \mathrm{O}_{4}$ have been monitored. It is observed that the ratio of product formation to $\mathrm{N}_{2} \mathrm{O}$ consumption is larger for $\mathrm{NO}_{2}$ and $\mathrm{N}_{2} \mathrm{O}_{4}$ combination products photochemically produced on the surface, compared to the gas phase.

3. The surface enhancement ratio, $S_{a}$, for the rate of formation of combination products from adsorbed $\mathrm{N}_{2} \mathrm{O}$ decomposition ranges from $\sim 1$ to $\sim 20$ for $\mathrm{NO}, \mathrm{NO}_{2}$ and $\mathrm{N}_{2} \mathrm{O}_{4}$ respectively. It is found that the enhancement ratio, $\mathrm{S}_{\mathrm{a}}$, increases with 
the product complexity. This indicates that products which require several radical recombination steps will be more enhanced in their yield by contact with the surface than will less complex combination products. It is proposed that the $\mathrm{SiO}_{2}$ surface enhances the rate of recombination of radical products by acting as a kinematic third body absorbing a portion of the exothermicity of the combination processes to make new chemical bonds.

4. The quantum yield (Q.Y.) for $\mathrm{N}_{2} \mathrm{O}$ photodecomposition has been measured for adsorbed $\mathrm{N}_{2} \mathrm{O}$ on high area $\mathrm{SiO}_{2}$ and is $\sim 0.005$. For $\mathrm{N}_{2} \mathrm{O}(\mathrm{g})$ the Q.Y. $=1.46$. The large decrease in the Q.Y. on $\mathrm{SiO}_{2}$ is attributed to Lyman- $\alpha$ absorption and scattering by the high area $\mathrm{SiO}_{2}$ powder.

5. The photodecomposition of isolated $\mathrm{Si}-\mathrm{OH}$ species on the $\mathrm{SiO}_{2}$ surface has been studied with Lyman- $\alpha$ irradiation, and a cross section of $3.1 \times 10^{-21} \mathrm{~cm}^{2}$ has been measured, assuming a radiation penetration depth of $104 \mathrm{~nm}$. This low Si$\mathrm{OH}$ damage cross section means that the kinetics of photodecomposition of adsorbed $\mathrm{N}_{2} \mathrm{O}$ held on $\mathrm{Si}-\mathrm{OH}$ sites is not significantly influenced by this small effect.

\subsection{References}

1. Benson, S.W., The Foundations of Chemical Kinetics 1960: McGraw Hill, New York.

2. Tielens, A.G.G.M., The Physics and Chemistry of the Interstellar Medium, 2005, Cambridge, UK: Cambridge University Press.

3. Vidali, G., et al., Catalytic Activity of Interstellar Grains: Formation of Molecular Hydrogen on Amorphous Silicates. Adv. Space. Res. , 2009. 43: p. 1291.

4. Pirronello, V., et al., Laboratory Synthesis of Molecular Hydrogen on Surfaces of Astrophysical Interest". Astrophys. J., 1997. 475: p. L69. 
5. Pirronello, V., et al., Measurement of Molecular Hydrogen Formation on Carbonaceous Grains. Astron. Astrophys. , 1999. 344: p. 681.

6. Hollenback, D., M.W. Werner, and E.E. Salpeter, Astrophys. J., 1971. 163: p. 165.

7. Hollenback, D. and E.E. Salpeter, Astrophys. J. , 1971. 163: p. 155.

8. Gerlich, D. and S. Horning, Chem. Rev., 1992. 92: p. 1509.

9. Puy, C.H.D., Pure Appl. Chem., 1989. 61: p. 693.

10. Garrod, R.T., S.L.W. Weaver, and E. Herbst, Astrophys. J., 1995. 682: p. 283.

11. Herbst, E. and E.F. van Dishoeck, Complex Organic Interstellar Molecules. Annu. Rev. Astron. Astrophys. , 2009. 47: p. 427.

12. Oberg, K.I., E.F. van Dishoeck, and H. Linnartz, Photodesorption of ices I: CO, $\mathrm{N}_{2}$, and $\mathrm{CO}_{2}$. Astron. Astrophys., 2009. 496: p. 281.

13. Oberg, K.I., et al., Formation rates of complex organics in UV irradiated $\mathrm{CH}_{3} \mathrm{OH}-$ rich ices: I. Experiments. Astron. Astrophys., 2009. 504(3): p. 891.

14. Watanabe, N., T. Shiraki, and A. Kouchi, The Dependence of $\mathrm{H}_{2} \mathrm{CO}$ and $\mathrm{CH}_{3} \mathrm{OH}$ Formation on the Temperature and Thickness of $\mathrm{H}_{2} \mathrm{O}-\mathrm{CO}$ Ice during the Successive Hydrogenation of CO. Astrophys. J., 2003. 588: p. L121.

15. Watanabe, N., et al., Dependence of the effective rate constants for the hydrogenation of $\mathrm{CO}$ on the temperature and composition of the surface. Planet Space Sci., 2006. 54: p. 1107.

16. Ziurys, L.M., et al., Detection of interstellar $\mathrm{N}_{2} \mathrm{O}$ : A new molecule containing an $\mathrm{N}$ O bond. Astrophysical Journal, 1994. 436(2): p. L181-L184.

17. Woon, D.E., Interstellar and Circumstellar Molecules. http://www.astrochymist.org/astrochymist_ism.html, 2012.

18. Zelikoff, M., K. Watanabe, and E.C.Y. Inn, Absorption Coefficients of Gases in the Vacuum Ultraviolet. Part II. Nitrous Oxide. J. Chem. Phys., 1953. 21: p. 1643.

19. Greiner, N.R., Photochemistry of N2O Essential to a Simplified Vacuum-Ultraviolet Actinometer. J. Chem. Phys., 1967. 47: p. 4373.

20. Black, G., et al., Quantum yields for the production of $\mathrm{O}(1 \mathrm{~S}), \mathrm{N}(2 \mathrm{D})$, and $\mathrm{N} 2(\mathrm{~A} 3 \Sigma+\mathrm{u})$ from the vacuum uv photolysis of $\mathrm{N}_{2}$ O. J. Chem. Phys., 1975. 62: p. 4266.

21. Lambert, H.M., et al., Photodissociation channels for $\mathrm{N}_{2} \mathrm{O}$ near $130 \mathrm{~nm}$ studied by product imaging. J. Chem. Phys., 2005. 122: p. 174304. 
22. Okabe, H., J. Chem. Phys., 1967. 47: p. 101.

23. Kroto, H.W., et al., Astrophys. J., 1987. 314: p. 352.

24. Blanco, A., et al., Astrophys. J., 1996. 472: p. 419.

25. Rajappan, M., et al., Photochemical Decomposition of $\mathrm{N}_{2} \mathrm{O}$ by Lyman-a Radiation: Scientific Basis for a Chemical Actinometer. J. Phys. Chem. A, 2010. 114: p. 3443.

26. Mueller, R., et al., $\mathrm{OH}$ surface density of $\mathrm{SiO}_{2}$ and $\mathrm{TiO}_{2}$ by thermogravimetric analysis. Langmuir 2003. 19: p. 160.

27. Xu, J., H.J. Jansch, and J.T. Yates, Jr., Cryogenic trick for enhanced cooling using liquid nitrogen. J. Vac. Sci. Technol. A, 1993. 11: p. 726.

28. Beebe, T.P. and J.T. Yates, Jr., Surf. Sci. , 1985. 148: p. 526.

29. Kiselev, A.V. and V.I. Lygin, Infrared Spectra of Surface Compounds1975: Wiley, New York.

30. Miller, T.M. and V.H. Grassian, Catal. Lett., 1997. 46: p. 213.

31. The adherence to Beer's law of the gas phase integrated absorbance $\int A d v$ has been verified for $\mathrm{N}_{2} \mathrm{O}$ in our experiments at $\approx 10$ Torr pressure (Ref. 25). Small differential changes in partial pressure of $\mathrm{N}_{2} \mathrm{O}$ may be accurately related to d $\int A d v$. Beer's law behavior is also found for $\mathrm{N}_{2} \mathrm{O}(\mathrm{g})$ mixed with $\mathrm{N}_{2}(\mathrm{~g})$ at high $\mathrm{N}_{2} / \mathrm{N}_{2} \mathrm{O}$ ratios to achieve line broadening. (a) [D. E. Burch and D. Williams, Appl. Opt. 1, 473 (1962)]; (b) in $\mathrm{N}_{2} \mathrm{O}$ ices of various measured thicknesses [D. Fulvio, B. Sivaraman, G. A. Baratta, M. E. Plumbo, and N. J. Mason, Spectrochim. Acta, Part A 72, 1007 (2009)].

32. E. V. Kondratieva, O. V. Manoilova, and A. A. Tsyganenko, Kinet. Catal. 49, 451 (2008). This paper compares the integrated infrared absorbances for $\mathrm{CO}(\mathrm{g})$ and $\mathrm{CO}(\mathrm{a})$ on $\mathrm{Si}-\mathrm{OH}$ groups on high area $\mathrm{SiO}_{2}$ similar to that used here. The two IR absorption coefficients differ by $\sim 40 \%$.

33. This is based on essentially full broadening of the rotational fine structure for the gas phase species at total pressures near 10 Torr and the assumption that rotational fine structure is absent for the adsorbed species at $71 \mathrm{~K}$.

34. In the measurement of $\mathrm{Sa}\left(\mathrm{N}_{2} \mathrm{O}_{4}\right)$, we could not measure any $\mathrm{N}_{2} \mathrm{O}_{4}$ formation in the gas phase. Therefore, the noise limit measured at the $\mathrm{N}_{2} \mathrm{O}_{4}$ mode frequency in the gas phase was used as an upper limit to estimate the relative rate of $\mathrm{N}_{2} \mathrm{O}_{4}$ formation. $\mathrm{Sa}\left(\mathrm{N}_{2} \mathrm{O}_{4}\right)$ therefore is a lower limit.

35. Groth, W.E. and H. Schierholz, Planet Space Sci., 1959. 1: p. 353. 
36. Tan, G.-L., M.F. Lemon, and R.H. French, J. Am. Chem. Soc., 2003. 86: p. 1885.

37. Herbst, E., J. Chem. Phys., 1979. 70: p. 2201. 


\title{
7 Chapter 7: Photochemical Decomposition of Adsorbed $\mathrm{N}_{2} \mathrm{O}$ on Simulated Silicate Interstellar Dust Grains using Lyman- $\alpha$ (10.2eV) Irradiation-The Role of Surface Functionalization of the $\mathrm{SiO}_{2}$ Substrate
}

\author{
Unpublished Manuscript
}

\subsection{Introduction}

Silicate-type dust grains are one of the most important types of dust grains in space[1]. It is generally accepted that the composition of the grain surface will have an influence on surface reactions as well as on the adsorption of molecules and ices[2, 3]. Lyman-a radiation $(10.2 \mathrm{eV})$ is a dominant source of radiation damage on grain surfaces in the astrophysical environment, causing molecules on surfaces and in ices to undergo chemical bond scission[4] to produce radical fragments which then undergo radical-radical or radical-molecule association reactions leading to new products. It is the production of these association products which carries part of the story of the evolution of higher molecular weight molecules in the interstellar medium.

In the case of the photochemical excitation of molecules on surfaces, leading to association reactions involving radical fragments, it is believed that these reactions are favored in adsorbed layers by the third-body effect in which excess momentum and energy of the combining radicals is absorbed by the surface at the moment of the associative formation of new chemical bonds [5]. By comparing the rate of the association reactions on a silica surface to those in the gas phase, it has been found that the surface kinetically favors the association products by a factor of $\sim 4$ and $\sim 20$ respectively for $\mathrm{NO}_{2}$ and $\mathrm{N}_{2} \mathrm{O}_{4}$ products made 
from the photodecomposition of adsorbed $\mathrm{N}_{2} \mathrm{O}$ on $\mathrm{SiO}_{2}$ [6]. This indicates the importance of the surface, acting as a third-body, in mediating associative recombination.

In addition to the general third-body effect on enhancing association reactions on surfaces there are three possible additional effects envisioned for photochemical decomposition of adsorbed molecules and the concomitant radical-radical association reactions on functionalized surfaces: (1). The association reaction can be negatively influenced by a cage effect, in which functional groups on the surface impede radical-radical reactions by molecular shielding[7, 8]; (2). Alternatively, the surface may promote a negative effect on reaction kinetics by quenching the primary photochemical reaction by the removal of electronic energy from the excited state prior to conversion of electronic energy into nuclear motion along the dissociation reaction coordinate for the excited molecule. Functional groups, acting as a spacer layer, would be expected to remove or reduce such quenching effects. (3). The primary direct photochemical dissociation process may also be enhanced by the production of unstable temporary negative ions due to electron transfer from the surface caused by electron-hole pair formation in the $\mathrm{SiO}_{2}$ under Lyman-a excitation. This is an indirect photochemical process[9]. Both the quenching effect and the indirect photochemical activation processes should depend on the ability of a surface, in contact with an adsorbed molecule, to exchange electronic energy or charge efficiently with the adsorbed molecule, causing the surface to produce either a negative effect on rate (quenching) or a positive effect on rate (indirect surface mediated photochemical process). In summary: (1). Surface functionalization would sterically impede association reactions (cage effect); (2).Surface functionalization would enhance photochemistry normally quenched by the clean surface; (3). Surface functionalization would impede photochemistry taking place via an indirect excitation process. 
The experiments to be described here are focused on the production of $\mathrm{N}_{2} \mathrm{O}$ photoproducts made from adsorbed $\mathrm{N}_{2} \mathrm{O}$ on $\mathrm{SiO}_{2}$ and chemically-modified $\mathrm{SiO}_{2}$ surfaces upon excitation with Lyman-a radiation at low temperatures. $\mathrm{N}_{2} \mathrm{O}$ is in fact one of the $\sim 170$ molecules discovered in interstellar space by radioastronomers [10]. It represents a model molecule for astrochemical studies. $\mathrm{N}_{2} \mathrm{O}$-Lyman-a photochemistry in the gas phase is well studied and relatively simple, and begins with the photochemical breaking of either the N-N bond or the $\mathrm{N}-\mathrm{O}$ bond[11, 12]. The fragments left behind react together to form $\mathrm{NO}, \mathrm{NO}_{2}$ and $\mathrm{N}_{2} \mathrm{O}_{4}$ by association reactions ( $\mathrm{NO}$ is also produced by direct dissociation). Studies of these processes have recently been reported, where the $\mathrm{N}_{2} \mathrm{O}$ photoreaction on a silica surface has been kinetically compared to the $\mathrm{N}_{2} \mathrm{O}$ photoreaction in the gas phase[6], finding the combination processes are enhanced on the $\mathrm{SiO}_{2}$ surface.

The experiment to be described here compares the rate of association product formation for Lyman-a induced $\mathrm{N}_{2} \mathrm{O}$ photodissociation on five types of $\mathrm{SiO}_{2}$ surfaces, either unfunctionalized bare $\mathrm{SiO}_{2}$ (called $\mathrm{Si}-\mathrm{O}-\mathrm{Si}$ ), or surfaces containing Si-OH functionalities, $\mathrm{Si}-\mathrm{OCH}_{3}$ functionalities, $\mathrm{Si}-\mathrm{O}-\mathrm{SiCl}_{3}$ functionalities, or $\mathrm{SiO}_{2}$ surfaces containing hundreds monolayers of amorphous $\mathrm{H}_{2} \mathrm{O}$ ice. Three scenarios may be contemplated in these experiments. (1): The presence of functionalities on the $\mathrm{SiO}_{2}$ surface kinetically impedes the association reactions, causing a reduction in the relative rate of associationproduct formation by steric screening ; (2): The $\mathrm{SiO}_{2}$ surface acts as a quencher for excited states, and functionalization of the $\mathrm{SiO}_{2}$ substrate should result in enhancement of the rate of association-product production, since quenching of the excitation process will be diminished as a result of the placement of a spacer layer between the adsorbed species and the surface; (3): The presence of functionalities on the $\mathrm{SiO}_{2}$ surface decouples the $\mathrm{N}_{2} \mathrm{O}$ from the surface, reducing the rate of charge transfer processes which result from electron-hole pair formation in the $\mathrm{SiO}_{2}$, thereby impeding the primary indirect excitation process for 
$\mathrm{N}_{2} \mathrm{O}$ and the concomitant association reactions. Our results suggest that model (2) is invalid and that either model (1) or model (3) are operative.

\subsection{Experimental}

\subsubsection{Apparatus}

The experiments are performed in an ultrahigh vacuum stainless steel cell (1.9 $\mathrm{L})$, which is pumped by a turbo pump and an ion pump, as discussed previously in Chapter 6 and shown in Figure 6.1. The base pressure of the mildly-baked cell is $1 \times 10^{-8}$ Torr. The $\mathrm{SiO}_{2}$ sample is pressed into a tungsten grid (using a laboratory hydraulic press with 5000 psi pressure) and then mounted on two $\mathrm{Ni}$ clamps, which may be cooled by liquid $\mathrm{N}_{2}$ in the reentrant rotatable support Dewar. By using the method of bubbling He gas through liquid $\mathrm{N}_{2}$, the grid could be cooled down to $75 \mathrm{~K}[13]$. The grid may also be heated electrically and the temperature is controlled to $\pm 0.1 \mathrm{~K}$ using a LabView program with high accuracy and reproducibility by monitoring the output of a type $\mathrm{K}$ thermocouple welded onto the upper central edge of the grid. The grid is mounted in the cell at $45^{\circ}$ to both Lyman- $\alpha$ light and the IR beam in order to measure the spectral changes during irradiation (Figure 6.3). Transmission IR spectra of the sample were taken by a Bruker Tensor 27 FTIR spectrometer with a dry-air purged external optical system and a liquid $\mathrm{N}_{2}$ cooled MCT detector. All spectra were measured at $2 \mathrm{~cm}^{-1}$ resolution using 256 scans and the background was automatically subtracted.

\subsubsection{Lyman- $\alpha$ Source}

The Lyman- $\alpha$ light is produced from a quartz microwave discharge lamp, using $1.00 \pm 0.02$ Torr of flowing $\mathrm{Ar}+\mathrm{H}_{2}\left(90 \% \mathrm{Ar}, 10 \% \mathrm{H}_{2}\right)$. The lamp was held by compression a Viton-O ring seal to a $\mathrm{MgF}_{2}$ cell window, which has an optical cutoff at $1100 \AA$ to remove Ar emission lines. The lamp has been actinometrically 
calibrated and the flux of photons is $2.7 \times 10^{14}$ photons $\mathrm{cm}^{-2} \mathrm{~s}^{-1}$ at the sample surface at $45^{\circ}[14]$.

\subsection{3 $\mathrm{SiO}_{2}$ Surface Modifications and $\mathrm{N}_{2} \mathrm{O}$ Adsorption}

(1) $\mathrm{Si}-\mathrm{OH}$ surface: The $\mathrm{SiO}_{2}$ sample (Aerosil 200, containing $2.8 \mathrm{Si}-\mathrm{OH}$ groups per $\mathrm{nm}^{2}$ of surface area) [15] is pressed into a round spot on the tungsten grid with a diameter of $0.7 \mathrm{~cm}$ and a thickness of $0.005 \mathrm{~cm}$. The $\mathrm{SiO}_{2}$ weighs $2.5 \times 10^{-3}$ $\mathrm{g}$ and the surface area is about $4400 \mathrm{~cm}^{2}$. Before experiments, the sample is preheated to $1000 \mathrm{~K}$ at a rate of $2 \mathrm{~K} / \mathrm{s}$ to remove possible impurities adsorbed on the surface.

(2) $\mathrm{Si}-\mathrm{OCH}_{3}$ surface: The $\mathrm{Si}-\mathrm{OH}$ surface above is methoxylated by reacting with methanol in situ [16]. In a typical procedure, 30 Torr of gas phase methanol is introduced into the cell, and then the sample is heated to $800 \mathrm{~K}$ at $1 \mathrm{~K} / \mathrm{s}$, and held at the temperature for $30 \mathrm{~min}$. The sample is then cooled down to room temperature and the cell is evacuated. After repeating this procedure 3 times, more than $98 \%$ of the $\mathrm{Si}-\mathrm{OH}$ groups are reacted to form $\mathrm{Si}-\mathrm{OCH}_{3}$ groups.

(3) Si-O-Si surface: The $\mathrm{SiOCH}_{3}$ surface above is heated to $1100 \mathrm{~K}$ to perform a thermal decomposition process. All $\mathrm{Si}-\mathrm{OCH}_{3}$ groups are decomposed and $\mathrm{Si}$ $\mathrm{OH}, \mathrm{SiH}_{\mathrm{x}}$ and $\mathrm{Si}-\mathrm{O}-\mathrm{Si}$ sites are formed [16]. By further heating the sample up to $1450 \mathrm{~K}$ for 1 hour, nearly $99 \% \mathrm{Si}-\mathrm{OH}$ groups are converted to Si-O-Si sites.

(4) $\mathrm{Si}-\mathrm{O}-\mathrm{SiCl}_{3}$ surface: $\mathrm{A}$ new $\mathrm{SiO}_{2}$ sample is prepared in the cell as in procedure (1). The cell is then filled with $3 \times 10^{-3} \mathrm{Torr}^{\mathrm{SiCl}}{ }_{4}$ gas and the sample is heated to $800 \mathrm{~K}$ and reacted for $10 \mathrm{~min}[17,18]$. Then the sample is cooled to room temperature and the cell is evacuated. By treating the sample twice with $\mathrm{SiCl}_{4}$, nearly all $\mathrm{Si}-\mathrm{OH}$ groups are converted to $\mathrm{Si}-\mathrm{O}-\mathrm{SiCl}_{3}$ groups as well as to some amount of Si-Cl groups via a direct chlorination process [19]. 
(5) $\mathrm{H}_{2} \mathrm{O} / \mathrm{SiO}_{2}$ surface: The $\mathrm{SiO}_{2}$ sample as in procedure (1) is cooled to $83 \mathrm{~K}$ with liquid $\mathrm{N}_{2}$. Gas phase $\mathrm{H}_{2} \mathrm{O}$ is then introduced into the cell and condensed on the $\mathrm{SiO}_{2}$ surface to form amorphous solid water with a thickness of about $300 \mathrm{~nm}$ [20].

After preparation of each surface, the sample is cooled down to $75 \mathrm{~K}$ (except $\mathrm{H}_{2} \mathrm{O} / \mathrm{SiO}_{2}$ experiment) and an IR spectrum of each surface is measured (Figure 7.1). $\mathrm{N}_{2} \mathrm{O}$ gas is introduced into the cell through a dosing tube which faces the sample. The amount of $\mathrm{N}_{2} \mathrm{O}$ adsorbed on the sample varies from one surface to another because of their different adsorption properties. $\mathrm{N}_{2} \mathrm{O}$ ice is also produced on some surfaces during dosing and it is removed by quickly heating the sample $(10 \mathrm{~K} / \mathrm{s})$ to $95 \mathrm{~K}$ and cooling back to $75 \mathrm{~K}$. The initial integrated absorbance of $\mathrm{N}_{2} \mathrm{O}$ on each surface is measured by IR spectroscopy and used to normalize the reaction rates measured on these surfaces.

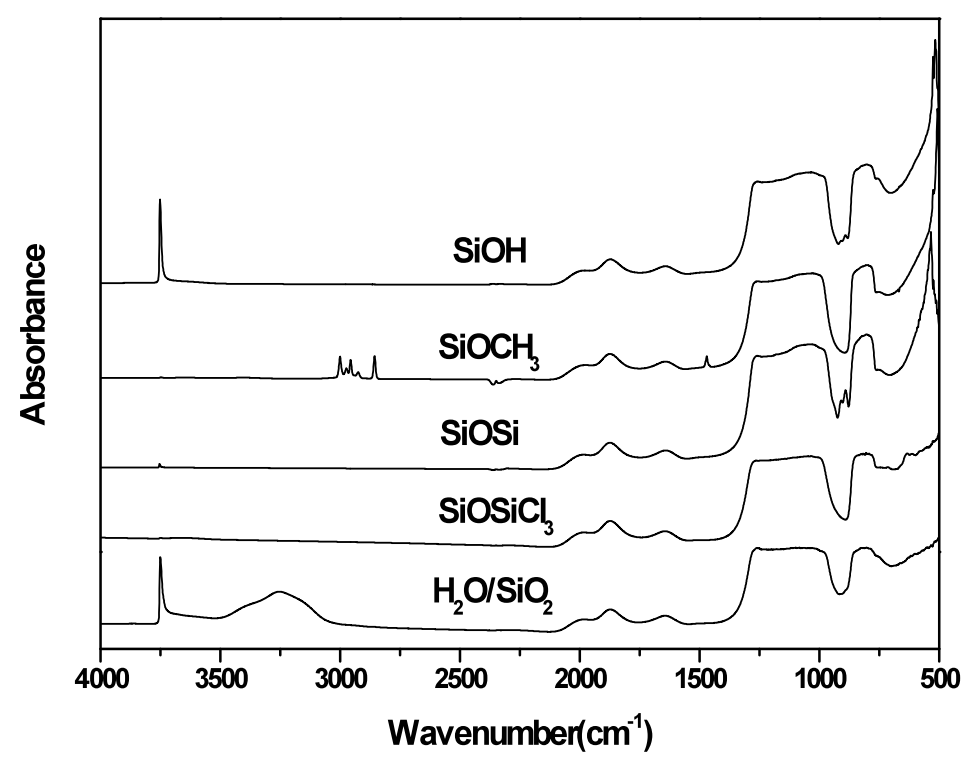

Figure 7.1: IR spectra of each modified $\mathrm{SiO}_{2}$ surface. 


\subsubsection{Stability of $\mathrm{Si}-\mathrm{OH}$ and $\mathrm{Si}-\mathrm{O}-\mathrm{SiCl}_{3}$ toward Lyman-a irradiation}

To confirm that the photodecomposition of adsorbed $\mathrm{N}_{2} \mathrm{O}$ is not related to radiation damage of $\mathrm{Si}-\mathrm{OH}$ and $\mathrm{Si}-\mathrm{O}-\mathrm{SiCl}_{3}$ functional groups, we performed photodecomposition experiments on those surfaces (without $\mathrm{N}_{2} \mathrm{O}$ ) in vacuum using Lyman-a radiation. Figure 7.2(a) shows the integrated $-\mathrm{OH}$ absorbance versus time, in which a very small amount of $\mathrm{Si}-\mathrm{OH}$ loss is observed at $300 \mathrm{~K}$. The cross section is estimated to be $Q_{\mathrm{Si}-\mathrm{OH}} \approx 3.1 \times 10^{-21} \mathrm{~cm}^{2}$ assuming the outer $1 / 5$ of the $\mathrm{SiO}_{2}$ surface receives Lyman-a radiation [6]. In Figure 7.2(b), the integrated absorbance of $\mathrm{Si}-\mathrm{Cl}$ remains constant with no-measurable change during 5 hours irradiation at $300 \mathrm{~K}$ and the dissociation cross section is considered to be zero to within our experimental accuracy. These cross sections are much smaller than for $\mathrm{N}_{2} \mathrm{O}$ depletion and therefore the destruction of $\mathrm{Si}-\mathrm{OH}$ and $\mathrm{Si}-\mathrm{O}-\mathrm{SiCl}_{3}$ groups by Lyman-a irradiation in the experiments may be neglected.
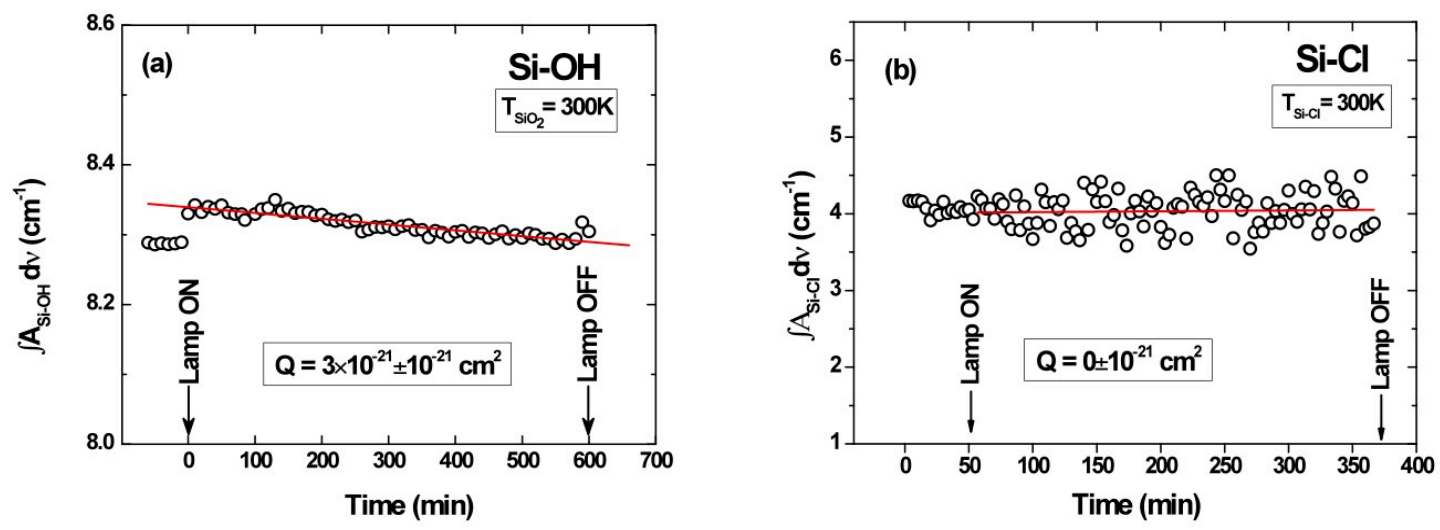

Figure 7.2: (a) the integrated $\mathrm{Si}-\mathrm{OH}$ absorbance versus time at $300 \mathrm{~K}$ during Lyman-a irradiation. (b) the integrated $\mathrm{Si}-\mathrm{Cl}$ absorbance versus time at $300 \mathrm{~K}$ during Lyman-a irradiation. 


\subsection{Results}

\subsubsection{Typical Spectral Observation of Photochemical Product Formation}

Figure 7.3 shows a typical spectral development of product IR bands during $\mathrm{N}_{2} \mathrm{O}$ photodecomposition in 2 hours on the $\mathrm{Si}-\mathrm{OH}$ surface. The growth of the NO stretching mode $\left(1876 \mathrm{~cm}^{-1}\right)$ and $\mathrm{NO}_{2}$ stretching mode $\left(1617 \mathrm{~cm}^{-1}\right)$ can be clearly observed during the irradiation [21]. For those experiments carried out on other functionalized surfaces, similar spectral developments have been observed. The spectral developments of $\mathrm{NO}$ and $\mathrm{NO}_{2}$ are clear and consistent, therefore we choose them to further investigate their behavior. It may also be seen that the $\mathrm{N}_{2} \mathrm{O}_{4}$ stretching mode $\left(1739 \mathrm{~cm}^{-1}\right)$ appears, but is not constantly developing in all cases. Due to its low IR intensity and low signal-to-noise ratio, $\mathrm{N}_{2} \mathrm{O}_{4}$ measurements have therefore not been included in these studies.

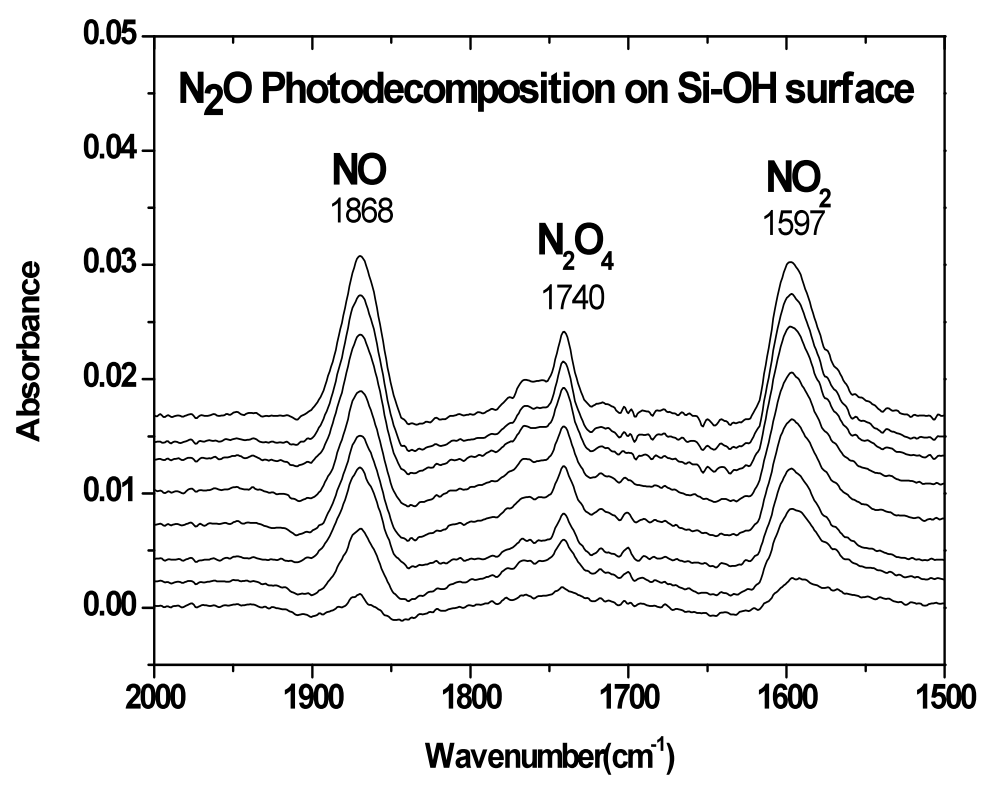

Figure 7.3: a typical spectral development of product IR bands during $\mathrm{N}_{2} \mathrm{O}$ photodecomposition on the $\mathrm{Si}-\mathrm{OH}$ surface. 


\subsubsection{Typical Kinetic Plots}

Figure 7.4 shows a typical quantitative measurement of the product formation rates on functionalized $\mathrm{SiO}_{2}$ surfaces. In each experiment, the adsorbed $\mathrm{N}_{2} \mathrm{O}$ has been kept in darkness on the $\mathrm{SiO}_{2}$ sample at $75 \mathrm{~K}$ for a 2 hours pre-photo stage before opening the internal shutter to the Lyman-a lamp. After 2 hours of stabilization, the rate of change of coverage due to thermal desorption of $\mathrm{N}_{2} \mathrm{O}$ has reached an acceptably low value to allow the photochemical experiments to proceed without being compromised by thermal desorption. The integrated absorbance above background in the spectral region of each product spectral band, measured in darkness, is calculated and plotted vs. time before irradiation, and we use this small slope as a small correction for a possible background effect during Lyman-a irradiation. Upon opening the internal shutter to the lamp, a steep initial slope of integrated absorbance can be observed for each product, $\mathrm{NO}, \mathrm{NO}_{2}$ and $\mathrm{N}_{2} \mathrm{O}_{4}$ and this slope, after correction for the small slope measured in darkness, is used as a kinetic measure of the rate of association product formation. 

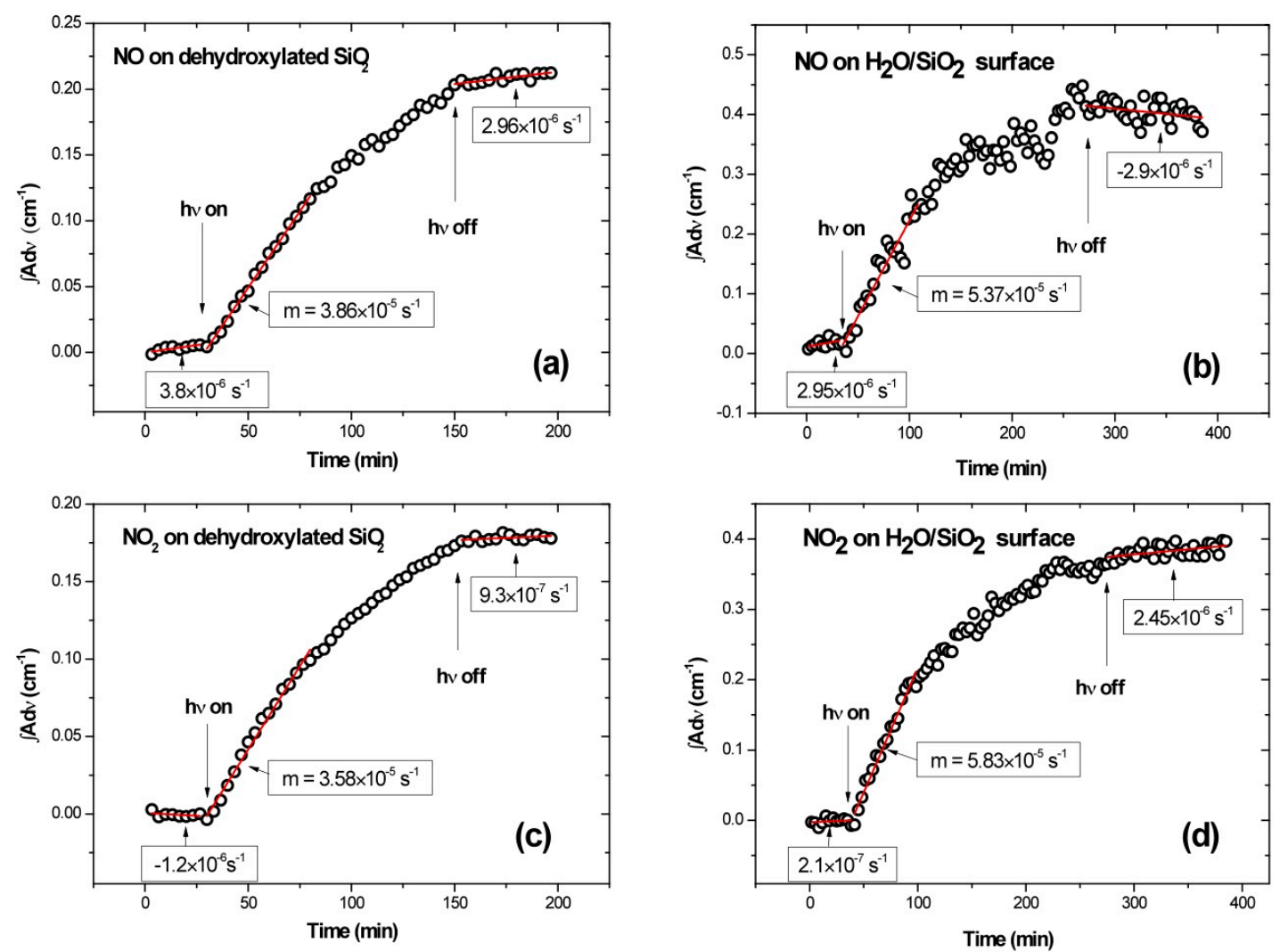

Figure 6

Figure 7.4: a typical quantitative measurement of the product formation rates on functionalized $\mathrm{SiO}_{2}$ surfaces.

\subsubsection{Reduced Initial Rate of Association Product Formation}

Since the initial absorbances of adsorbed $\mathrm{N}_{2} \mathrm{O}$ on the different functionalized surfaces are not the same, a reduced initial rate of product formation on each surface has been calculated to reflect the actual relative rate of photodecomposition. The reduced initial rate is determined by taking the ratio of measured initial rate of product formation $d \int A_{\text {product }}^{0} d v / d t$ to the integrated absorbance of $\mathrm{N}_{2} \mathrm{O}$ at the initial $\mathrm{N}_{2} \mathrm{O}$ coverage, $\int A_{\mathrm{N}_{2} \mathrm{O}}^{0} d v$. 
Reduced initial rate $=\hat{r}_{\text {product }}=\frac{-d \int A_{\text {product }}^{0} d v / d t}{\int A_{N_{2} O}^{0} d v}$

The slopes obtained in each kinetic plot are presented in Table 1, as well as the initial $\mathrm{N}_{2} \mathrm{O}$ integrated absorbance in each experiment, and the calculated reduced initial rates of product formation are also shown. These normalized photo-production rates are also plotted with error bars in Figure 7.5, from which we can see that these rates vary within a $\sim 3$ fold range, with the rate of association product formation on $\mathrm{Si}-\mathrm{O}-\mathrm{Si}$ surfaces being statistically larger than on the functionalized surfaces. A student t-test analysis of the data for each type of surface has been performed to determine the statistical significance of the higher rate of association product formation on the unfunctionalized $\mathrm{Si}-\mathrm{O}-\mathrm{Si}$ surface compared to the average of the four rates on the functionalized $\mathrm{SiO}_{2}$ surfaces. For both $\mathrm{NO}$ and $\mathrm{NO}_{2}$ association products, the confidence level that their individual rate of formation on the $\mathrm{Si}-\mathrm{O}-\mathrm{Si}$ unfuctionalized surface is statistically greater than the average on the four functionalized surfaces is greater than $95 \%$. Considering that both $\mathrm{NO}$ and $\mathrm{NO}_{2}$ formation rates are more that $50 \%$ higher on the unfunctionalized $\mathrm{Si}-\mathrm{O}-\mathrm{Si}$ surface than on all the functionalized surfaces with a $95 \%$ confidence for each of the two measurements, there is high confidence that the two results, considered together, are statistically meaningful in indicating that the rate of the association reactions on the bare $\mathrm{Si}-\mathrm{O}-\mathrm{Si}$ surfaces exceeds the rate on the functionalized surfaces, considered singly or as a group. 

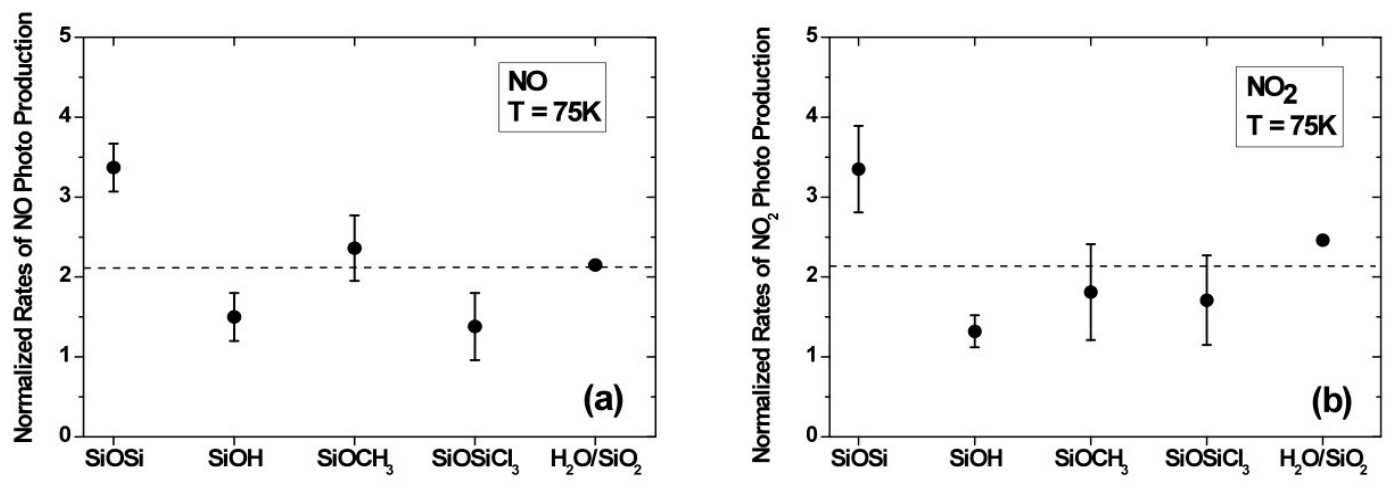

Figure 7.5: normalized photo-production rates with error bars on each functionalized $\mathrm{SiO}_{2}$ surface.

Table 7.1. Kinetic data and reduced initial rates for $\mathrm{N}_{2} \mathrm{O}$ photodecomposition on different functionalized surfaces

\begin{tabular}{|c|c|c|c|c|c|}
\hline \multirow[t]{2}{*}{$\begin{array}{l}\text { Functionalized } \\
\text { Surfaces }\end{array}$} & \multirow[t]{2}{*}{$\begin{array}{l}\int A_{\mathrm{N}_{2} \mathrm{O}}^{0} d v \\
\text { and } \\
\text { Temperature } \\
\text { during photo- } \\
\text { decompositon }\end{array}$} & \multicolumn{2}{|c|}{$\begin{array}{l}\text { Averaged roduct } \\
\text { formation rate } \\
d \int A_{\text {product }}^{0} d v / d t\left(\mathrm{~s}^{-1}\right)\end{array}$} & \multicolumn{2}{|c|}{$\begin{array}{l}\text { Reduced product formation } \\
\text { rate } \\
\hat{r}_{\text {product }}=\frac{-d \int A_{\text {product }}^{0} d v / d t}{\int A_{N_{2} O}^{0} d v}\end{array}$} \\
\hline & & NO & $\mathrm{NO}_{2}$ & NO & $\mathrm{NO}_{2}$ \\
\hline Raw $\mathrm{SiO}_{2}$ & $2.51,75 \mathrm{~K}$ & $3.76 \times 10^{-5}$ & $3.33 \times 10^{-5}$ & $-1.50 \times 10^{-5}$ & $-1.33 \times 10^{-5}$ \\
\hline $\begin{array}{l}\text { Methoxylated } \\
\mathrm{SiO}_{2}\end{array}$ & $1.02,75 \mathrm{~K}$ & $2.41 \times 10^{-5}$ & $1.85 \times 10^{-5}$ & $-2.36 \times 10^{-5}$ & $-1.81 \times 10^{-5}$ \\
\hline $\begin{array}{l}\text { Dehydroxylated } \\
\mathrm{SiO}_{2}\end{array}$ & $1.01,75 \mathrm{~K}$ & $3.40 \times 10^{-5}$ & $3.38 \times 10^{-5}$ & $-3.37 \times 10^{-5}$ & $-3.35 \times 10^{-5}$ \\
\hline $\begin{array}{l}\text { Chlorinated } \\
\mathrm{SiO}_{2}\end{array}$ & $1.45,75 \mathrm{~K}$ & $2.00 \times 10^{-5}$ & $2.48 \times 10^{-5}$ & $-1.38 \times 10^{-5}$ & $-1.71 \times 10^{-5}$ \\
\hline $\mathrm{H}_{2} \mathrm{O} / \mathrm{SiO}_{2}$ & $2.36,83 \mathrm{~K}$ & $5.08 \times 10^{-5}$ & $5.81 \times 10^{-5}$ & $-2.15 \times 10^{-5}$ & $-2.46 \times 10^{-5}$ \\
\hline
\end{tabular}




\subsection{Discussion}

Three phenomena have been envisioned as possible factors in controlling the reduced rate of association product formation as outlined in the Introduction. We examine each of these phenomena in more detail below:

\subsubsection{Steric Screening of Association Reaction by Surface Functionalities}

Schmidt and Gerber[8] have recently reexamined the "cage effect" first postulated by Franck and Rabinovich[7]. For association reactions, solvent cages retard the kinetics of association reactions in ice clusters, etc. When the ice cage melts, theory shows that an association reaction rate can be enhanced by orders of magnitude as molecular motion of the cage molecules opens channels to reaction. In the limit of a single molecule in the vicinity of two radical species the opposite effect may occur where the single molecule acts as a third body and enhances the recombination reaction. We conclude that our data, showing the enhanced radical-radical reactivity on the bare $\mathrm{SiO}_{2}$ surface, is consistent with a cage effect caused by functional groups on the functionalized $\mathrm{SiO}_{2}$ surfaces which sterically impede association reactions.

\subsubsection{Quenching of the Electronically Excited States}

The primary photodecomposition processes for $\mathrm{N}_{2} \mathrm{O}$ with Lyman-a radiation is initiated by the breaking of either the $\mathrm{N}-\mathrm{O}$ bond or the $\mathrm{N}-\mathrm{N}$ bond as shown in equation (1) and (2).

$$
\mathrm{N}_{2} \mathrm{O}+\mathrm{hv}(10.2 \mathrm{eV}) \rightarrow \mathrm{N}_{2}+\mathrm{O}
$$

or,

$$
\mathrm{N}_{2} \mathrm{O}+\mathrm{hv}(10.2 \mathrm{eV}) \rightarrow \mathrm{NO}+\mathrm{N}
$$


The fragment species produced photochemically may exist in different electronic states[22, 23]. The rapid deactivation of these electronically-excited species can be expected at an interface. In the case of the unfunctionalized Si-O-Si surface, because of the absence of a spacer layer, the excited species can contact the $\mathrm{SiO}_{2}$ substrate directly, and one might expect that transfer of electronic energy would be favored, leading to retardation of the association reactions. In fact the opposite is true from our measurements summarized in Figure 7.5, where the association reactions are enhanced on the unfunctionalized $\mathrm{SiO}_{2}$ surface. This suggests that a model involving electronic interaction of $\mathrm{SiO}_{2}$ surfaces with excited surface species, leading to quenching effects, is not appropriate since the expected quenching in this model is not seen.

\subsubsection{Indirect Photochemistry Involving Electronic Excitation of $\mathrm{SiO}_{2}$}

Photochemical studies on surfaces were revolutionized in the 1970's by surface scientists who discovered that photo-excitation on metal or semiconductor surfaces could take place not only by direct excitation of the adsorbed molecule, but also by excitation of the underlying surface[9]. Electronhole pairs so produced were found to influence adsorbed species by hot electron transport from the substrate to the adsorbed molecule when an empty orbital was available on the molecule, leading to the production of a temporary negative ion with a short lifetime. This ion then underwent dissociation by a dissociative electron attachment (DEA) process. The process of indirect photochemistry is schematically shown in Figure 7.6, contrasted from the direct excitation process, also shown. High cross-section indirect photoexcitation processes are widely observed in surface science experiments, and we should be on the lookout for the indirect photochemical excitation process in systems of astrochemical interest. 
DIRECT ELECTRONIC EXCITATION

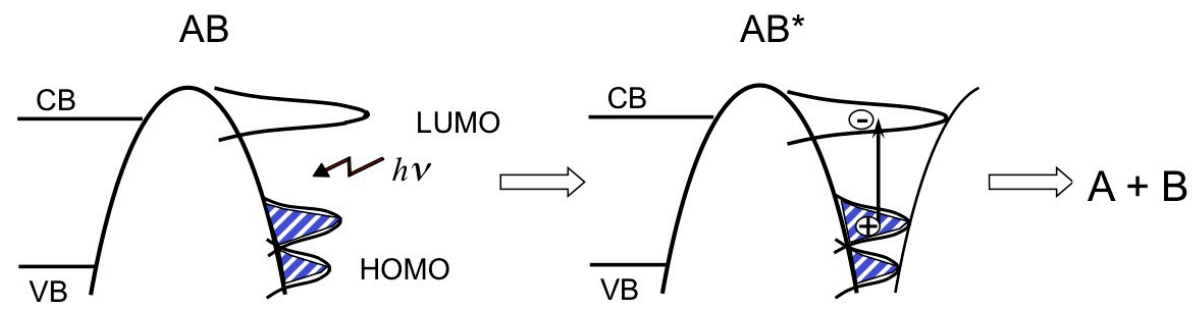

INDIRECT ELECTRONIC EXCITATION

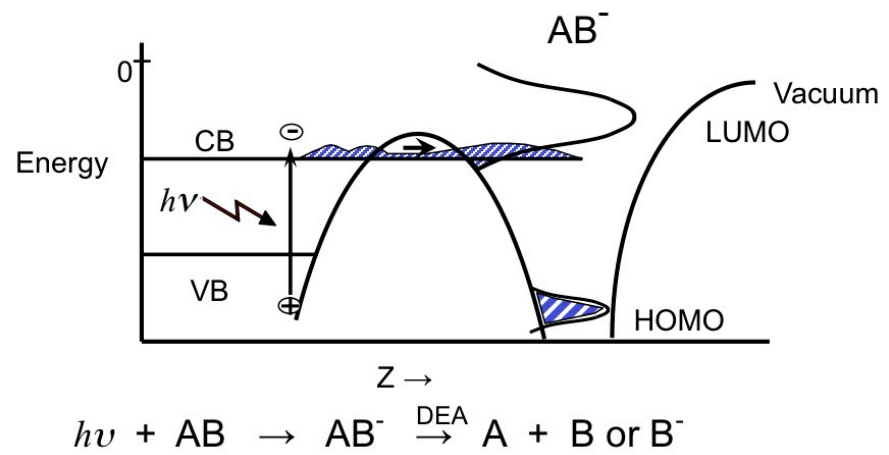

Figure 7.6: Schematics of direct and indirect photochemistry processes.

For $\mathrm{SiO}_{2}$, the bandgap is in the range of $8-9 \mathrm{eV}$ [24]. Therefore it is thermodynamically possible for Lyman-a irradiation of $\mathrm{SiO}_{2}$ to excite an electron into the conduction band where it can participate in an indirect photochemical process. Excitation from impurity states residing in the band gap are also possible [25]. A bare $\mathrm{SiO}_{2}$ surface would therefore be expected to exhibit an indirect excitation process, accompanied by direct excitation of the adsorbed molecule, causing enhanced dissociation followed by subsequent radical-radical association processes. Placing a spacer layer on the $\mathrm{SiO}_{2}$ would be expected to diminish the indirect process and the data in Figure 7.5 therefore indicate that indirect excitation of adsorbed $\mathrm{N}_{2} \mathrm{O}$ molecules is therefore a possible interpretation to explain the added photoreactivity of $\mathrm{N}_{2} \mathrm{O}$ on the bare $\mathrm{SiO}_{2}$ surface. 


\subsection{Summary and Conclusions}

Experiments with the photodissociation of adsorbed $\mathrm{N}_{2} \mathrm{O}$ on $\mathrm{SiO}_{2}$ and functionalized- $\mathrm{SiO}_{2}$ surfaces using Lyman-a $(10.2 \mathrm{eV})$ radiation have shown that four types of surface functionalization of the $\mathrm{SiO}_{2}$ surface cause a $\sim 50 \%$ depletion in the rate of association product $\left(\mathrm{NO}\right.$ and $\left.\mathrm{NO}_{2}\right)$ formation at $75 \mathrm{~K}$ compared to reaction on the unfunctionalized $\mathrm{SiO}_{2}$ surface. The surface functionalization involved the production of $\mathrm{Si}-\mathrm{OH}$ species, $\mathrm{Si}-\mathrm{OCH}_{3}$ species, Si$\mathrm{O}-\mathrm{SiCl}_{3}$ species, and a thick layer of amorphous $\mathrm{H}_{2} \mathrm{O}$ ice. The functionalization was monitored by infrared spectroscopy, as was the photoproduct formation from adsorbed $\mathrm{N}_{2} \mathrm{O}$. Control experiments showed that the functional groups were very insensitive to Lyman-a exposure and were not involved in the surface photochemistry.

Two models are consistent with these findings and at present are not separable:

1. Functional groups (and $\mathrm{H}_{2} \mathrm{O}$ ice) produce a "cage effect" around radical species derived from $\mathrm{N}_{2} \mathrm{O}$, hindering their association.

2. Clean $\mathrm{SiO}_{2}$ surfaces make a contribution to $\mathrm{N}_{2} \mathrm{O}$ photodissociation in which hot electrons produced in the $\mathrm{SiO}_{2}$ attach to adsorbed $\mathrm{N}_{2} \mathrm{O}$ molecules, leading to dissociative electron attachment and producing radical intermediates which then associate. This excitation route accompanies a direct photodissociation process.

The studies are inconsistent with the preferential quenching of electronically excited surface species by the unfunctionalized, bare $\mathrm{SiO}_{2}$ surface. 


\subsection{Reference:}

1. Sargent, B.A., et al., Silica in Protoplanetary Disks. Astrophysical Journal, 2009. 690(2): p. 1193-1207.

2. Tielens, A.G.G.M., The Physics and Chemistry of the Interstellar Medium. 2005, Cambridge, UK: Cambridge University Press.

3. Pirronello, V., et al., Measurement of Molecular Hydrogen Formation on Carbonaceous Grains. Astron. Astrophys., 1999. 344: p. 681.

4. van Dishoeck, E.F., B. Jonkheida, and M.C.van Hemert, Photoprocesses in protoplanetary disks. Faraday Discussions, 2006. 133: p. 231.

5. Vidali, G., et al., Catalytic Activity of Interstellar Grains: Formation of Molecular Hydrogen on Amorphous Silicates. Adv. Space. Res. , 2009. 43: p. 1291.

6. Rajappan, M., C. Yuan, and J.T. Yates, Lyman-a driven molecule formation on $\mathrm{SiO}_{2}$ surfaces - connection to astrochemistry on dust grains in the interstellar medium. J. Chem. Phys, 2011. 134: p. 064315.

7. Frank, J. and E. Robinovitch, Trans. Faraday Soc, 1934. 30: p. 120.

8. Schmidt, B. and R.B. Gerber, Reactive Collisions as a Signature for Meltinglike Transitions in Clusters. Phys. Rev. Lett., 1994. 72: p. 2490-2493.

9. Yates, J.T. and H. Petek, Introduction: Photochemistry and Photophysics on surfaces. Chem. Rev., 2006. 106: p. 4113.

10. Ziurys, L.M., et al., Detection of interstellar $\mathrm{N}_{2} \mathrm{O}$ : A new molecule containing an $\mathrm{N}$ O bond. Astrophysical Journal, 1994. 436(2): p. L181-L184.

11. Zelikoff, M., K. Watanabe, and E.C.Y. Inn, Absorption Coefficients of Gases in the Vacuum Ultraviolet. Part II. Nitrous Oxide. J. Chem. Phys., 1953. 21: p. 1643.

12. Greiner, N.R., Photochemistry of N2O Essential to a Simplified Vacuum-Ultraviolet Actinometer. J. Chem. Phys., 1967. 47: p. 4373.

13. Xu, J., H.J. Jansch, and J.T. Yates, Cryogenic trick for enhanced cooling using liquid nitrogen. J. Vac. Sci. Technol. A, 1993. 11: p. 726.

14. Rajappan, M., et al., Photochemical Decomposition of $\mathrm{N}_{2} \mathrm{O}$ by Lyman-a Radiation: Scientific Basis for a Chemical Actinometer. J. Phys. Chem. A, 2010. 114: p. 3443.

15. Mueller, R., et al., $\mathrm{OH}$ surface density of $\mathrm{SiO}_{2}$ and $\mathrm{TiO}_{2}$ by thermogravimetric analysis. Langmuir 2003. 19: p. 160. 
16. Wovchko, E.A., et al., Active Sites on $\mathrm{SiO}_{2}$ : Role in $\mathrm{CH}_{3} \mathrm{OH}$ Decomposition. Langmuir, 1995. 11: p. 2592.

17. Hair, M.L. and W. Hertl, Chlorination of Silica Surfaces. J. Phys. Chem., 1973. 77(17): p. 2070.

18. McDaniel, M.P., Surface Halides of Silica. 1. Chloride. J. Phys. Chem., 1981. 85: p. 532.

19. Lang, S.J. and B.A. Morrow, Infrared Spectra of Chlorinated Silica. J. Phys. Chem., 1994. 98: p. 13314.

20. Rajappan, M., C. Yuan, and J.T. Yates, Lyman-a Induced Phase Transition in Ice at 83K: Photochemical Conversion of Amorphous Solid Water to Hexagonal-ice on a SiO2 Surface. 2011.

21. Burch, D.E. and D. Williams, Total Absorptance by Nitrous Oxide Bands in the Infrared. Applied Optics,, 1962. 1(4): p. 473.

22. Black, G., et al., Quantum yields for the production of $O(1 S), N(2 D)$, and N2(A $3 \Sigma+u)$ from the vacuum uv photolysis of N2O. J. Chem. Phys., 1975. 62: p. 4266.

23. Lambert, H.M., et al., Photodissociation channels for $\mathrm{N} 2 \mathrm{O}$ near $130 \mathrm{~nm}$ studied by product imaging. J. Chem. Phys., 2005. 122: p. 174304.

24. Laughlin, R.B., Optical absorption edge of SiO2. Phys. Rev. B, 1980. 22: p. 3021.

25. Pantelides, S.T., The Electronic Structure of Impurities and Defects In $\mathrm{SiO}_{2}$ Thin Solid Films, 1982. 89: p. 103. 


\section{Appendix I: Spectroscopic Observations of the Dynamics of the Displacement of Physically Adsorbed Molecules- CO on $\mathrm{C}_{60}$}

Unpublished Manuscript

\subsection{Introduction}

The physical adsorption of molecules on the surfaces under equilibrium conditions provides the basis for a wide range of sorption technologies used to purify gas streams as well as for the adsorption of contaminants from liquids containing dissolved substances. High area carbon surfaces are often used for such technical adsorption processes[1]. Physical adsorption also provides the basis for the determination of the surface area of high area powdered materials through isotherm fits[2, 3], including the widely used BET method[4]. Under adsorption conditions the adsorbed layer exists in dynamic equilibrium with the gas or liquid phase. In this work, we probe the effect of competing physisorbed gases on the equilibrium layer and report observations and calculations which give insight into the dynamics of displacement of one physisorbed molecule by another. Such displacement processes are important in technological sorption processes since displacement of a one sorbate by another sorbate molecule could defeat the purpose of the adsorption process.

The dynamical displacement process may be similar to the mechanism of collision induced desorption and dissociation of chemisorbed adsorbates on metal substrates, which has been studied in the past. Yates and Goodman[5] found that the presence of $\mathrm{CO}$ gas enhanced the desorption rate for $\mathrm{CO}$ on $\mathrm{Ni}(100)$, and they believed it is a result of CO-CO repulsive lateral interaction lowering the $\mathrm{CO}$ chemisorption energy. The involvement of physisorbed $\mathrm{CO}$ 
species in forcing the replacement of chemisorbed $\mathrm{CO}$ was excluded. The dynamical process as well as the $\mathrm{CO}$ adsorption energy on $\mathrm{Ni}(100)$ have also been studied by others, confirming the repulsive model [6]. However, these studies are related to $\mathrm{CO}$ chemisorption whereas the present work concerns the physisorption of $\mathrm{CO}$.

The $\mathrm{C}_{60}$ molecule provides a convenient high area carbon surface for physical adsorption studies. Its geometrical surface area of $1287 \mathrm{~m}^{2} \cdot \mathrm{g}^{-1}$ consists of two parts-that on the geometrical outer surface and that buried in the crystal structure of the solid $\mathrm{C}_{60}$. We have used $\mathrm{CO}$ as the physisorbed molecule of interest since it is active in the infrared spectral region permitting its observation at the fractional monolayer level. Our spectroscopic measurements of the infrared lineshape of physisorbed $\mathrm{CO}$ indicates that under the low pressure conditions of this experiment, only surface-bound $\mathrm{CO}$ is being adsorbed. Much higher pressures of $\mathrm{CO}$ are needed to occupy the interior sites which exist between $\mathrm{C}_{60}$ molecules in solid crystalline $\mathrm{C}_{60}$ [7]. The physisorption of $\mathrm{CO}$ on the surface of solid $\mathrm{C}_{60}$ at cryogenic temperatures was first observed by Folman and colleagues[8, 9] using transmission IR spectroscopy to study adsorption on an evaporated $\mathrm{C}_{60}$ film. A strong $v(\mathrm{CO})$ absorption band was observed at $2128 \mathrm{~cm}^{-1}$ at temperatures near $77 \mathrm{~K}$.

The physical adsorption of other gases on $\mathrm{C}_{60}$ surfaces has been studied. For example, Trasca et al. [10] studied noble gases adsorption on a $\mathrm{C}_{60}$ monolayer theoretically and experimentally, and phase transitions on the surface have been found to occur involving different site locations on the hexagonally close-packed $\mathrm{C}_{60}$ surface. The energy changes associated with these transitions are small compared to the adsorption energy.

The general principles reported here are likely to apply also to technological carbon surfaces. The use of a highly uniform $\mathrm{C}_{60}$ substrate for these studies 
permits one to see details of behavior which would be difficult to measure and interpret on more heterogeneous carbon surfaces.

\subsection{Experimental}

The experiments were carried out in an ultrahigh vacuum stainless steel cell $(1.9 \mathrm{~L})$, with a base pressure of $1 \times 10^{-8}$ Torr. As shown in Figure 8.1, the $\mathrm{C}_{60}$ sample was prepared using an evaporation method. In order to support the $\mathrm{C}_{60}$ sample, pre-dried $\mathrm{KBr}$ powder was pressed into a tungsten grid to make an IR transparent support, and then the grid was mounted on two Ni clamps [11]. This grid can be cooled by $\mathrm{N}_{2}(l)$ held in a rotatable support Dewar. A type $\mathrm{K}$ thermocouple was welded onto the grid. The grid could be electrically heated and the temperature can be controlled to $\pm 0.1 \mathrm{~K}$ using a LabView program which monitors the output of the thermocouple. The $\mathrm{C}_{60}$ powder was obtained from MER Corporation. The powder was transferred into a Macor crucible that faces the grid containing the $\mathrm{KBr}$ support, as shown in Figure 8.1. The crucible was electrically heated with a coiled tungsten wire and the $\mathrm{C}_{60}$ temperature was measured by an internal type $\mathrm{K}$ thermocouple. 

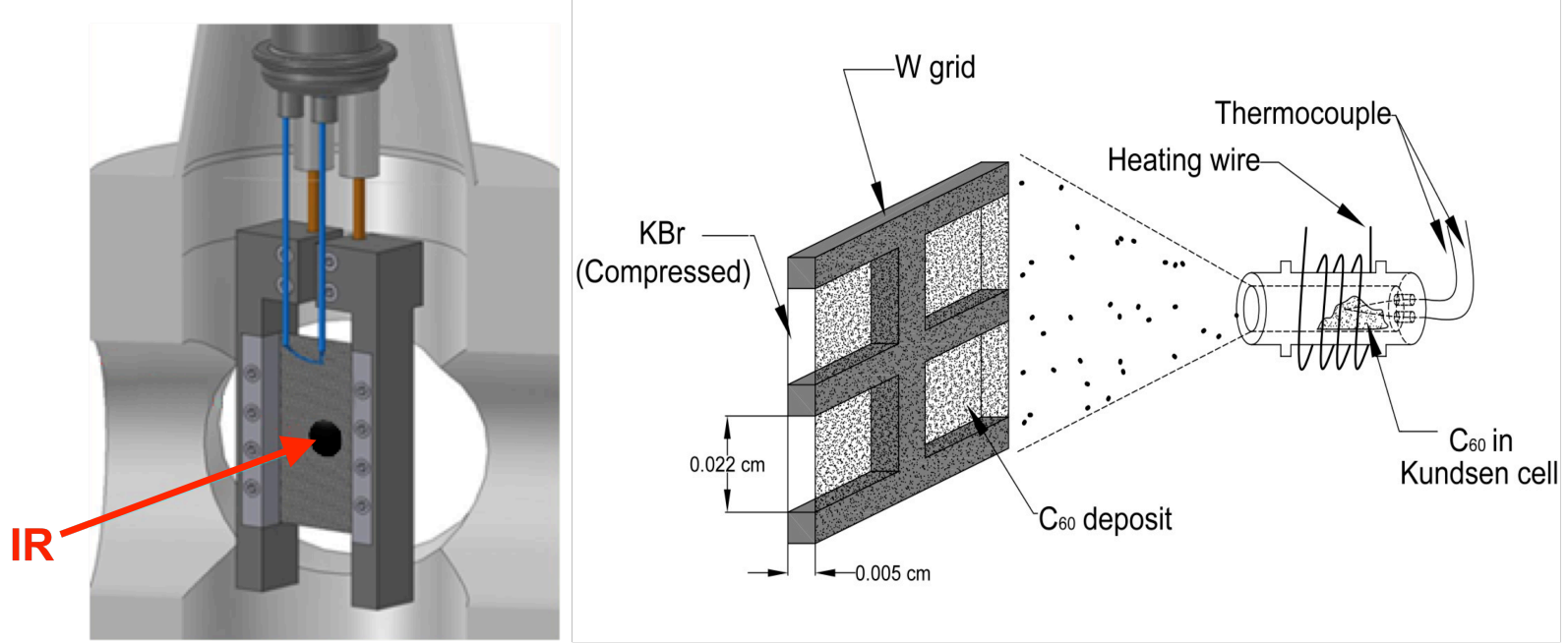

(a)

Figure 8.1. (a) The configuration of $\mathrm{KBr}$ support pressed on a tungsten grid in the IR vacuum cell. (b) The evaporation process of $\mathrm{C}_{60}$ onto the $\mathrm{KBr}$ support.

Before evaporation, both the $\mathrm{KBr}$ support and $\mathrm{C}_{60}$ powder were heated to $400 \mathrm{~K}$ and degassed for 1 hour. Then the $\mathrm{KBr}$ support was cooled to $82 \mathrm{~K}$ using $\mathrm{N}_{2}(\mathrm{I})$ and rotated to face the crucible while the $\mathrm{C}_{60}$ inside the crucible was heated to $673 \mathrm{~K}$ in high vacuum. At this temperature, $\mathrm{C}_{60}$ sublimes from the crucible and is deposited onto the $\mathrm{KBr}$ support surface. IR measurement of the sample clearly shows the growth of the $C_{60}$ layer. Four fundamental modes of $C_{60}$ at 1431,1182 , 576 and $526 \mathrm{~cm}^{-1}$ develop, as well as some small combination modes [12, 13]. The thickness of $C_{60}$ is estimated to be $(4.5 \pm 1) \times 10^{3}$ monolayers of $C_{60}$ molecules from its integrated IR absorbance [14]. In another method, we use the period of the interference fringes in the $C_{60}$ spectra over the frequency range of 2400-1000 $\mathrm{cm}^{-1}$ to estimate a $\mathrm{C}_{60}$ thickness of $5 \times 10^{3}$ monolayers[7, 13].

The $\mathrm{C}_{60}$ sample was annealed in vacuum at room temperature overnight after evaporation. It was then cooled to the desired temperature during each experiment. $\mathrm{CO}$ and other noble gases were introduced into the cell through a precision variable leak valve. The pressure of the cell was recorded in three ways: a cold cathode measures pressure below $1 \times 10^{-4}$ Torr; a sensitive Baratron 
gauge measures pressure between $1 \times 10^{-4}$ and 0.02 Torr, with a resolution of $10^{-6}$ Torr; then a less sensitive Baratron measures pressure above 0.02 Torr, with the a resolution of 0.001 Torr. Transmission IR spectra of the sample were measured by a Bruker Tensor 27 FTIR spectrometer with a $\mathrm{N}_{2}(l)$ cooled MCT detector. All spectra were measured at $4 \mathrm{~cm}^{-1}$ resolution using 128 scans and the background spectrum was automatically subtracted. The entire spectrometer optical path is purged with dry, $\mathrm{CO}_{2}$ free air.

\subsection{Results and Discussion}

\subsubsection{Adsorption of $\mathrm{CO}$ on $\mathrm{C}_{60}$}

Figure 8.2 shows the progress of the physical adsorption of $\mathrm{CO}$ on $\mathrm{C}_{60}$. $\mathrm{A}$ single band at $2129 \mathrm{~cm}^{-1}$ develops at constant wavenumber as the coverage increases at $110 \mathrm{~K}$. The spectral FWHM, corrected for the $4 \mathrm{~cm}^{-1}$ spectrometer resolution, is $6.9 \mathrm{~cm}^{-1}$, while Meijer and colleagues reported a $v(C O)=2127 \mathrm{~cm}^{-1}$ with a band $\mathrm{FWHM}=2.5 \mathrm{~cm}^{-1}$ for intercalated $\mathrm{CO}$ on $\mathrm{C}_{60}$ at $77 \mathrm{~K}$ when working at a spectrometer resolution $=0.2 \mathrm{~cm}^{-1}$ [7]. Using the integrated absorbance of $v(\mathrm{CO})$ and assuming that interactional effects between $\mathrm{CO}$ molecules do not seriously affect the infrared absorption coefficient, Figure 8.2 shows that a Langmuir isotherm fits the data well. The reversible behavior of the adsorbed $\mathrm{CO}$ is strictly related to the CO pressure with no hysteresis effects, consistent with the expected behavior for a homogeneous surface. Using the known IR absorbance coefficient for $\mathrm{CO}(\mathrm{g})[15,16]$, we estimate that the saturation coverage of $\mathrm{CO}$ on $\mathrm{C}_{60}$ in our experiment involves a $\mathrm{CO}$ saturation coverage on the outer surface of the $\mathrm{C}_{60}$ of $6 \times 10^{20} \mathrm{CO}$ molecules $\cdot \mathrm{m}^{-2}$. This coverage of $\mathrm{CO}$ indicates the high degree of porosity of the evaporated $\mathrm{C}_{60}$ film in the first $\sim 100$ $\mathrm{C}_{60}$ layers near the outer surface, which allows the occupancy of $\mathrm{CO}$ on the outer sites. The outer region available to $\mathrm{CO}$ adsorption is about $10^{-2}$ of the $\mathrm{C}_{60}$ film 
thickness. The interior sites investigated by Meijer et al.[7] are not populated at the low CO pressures employed here.

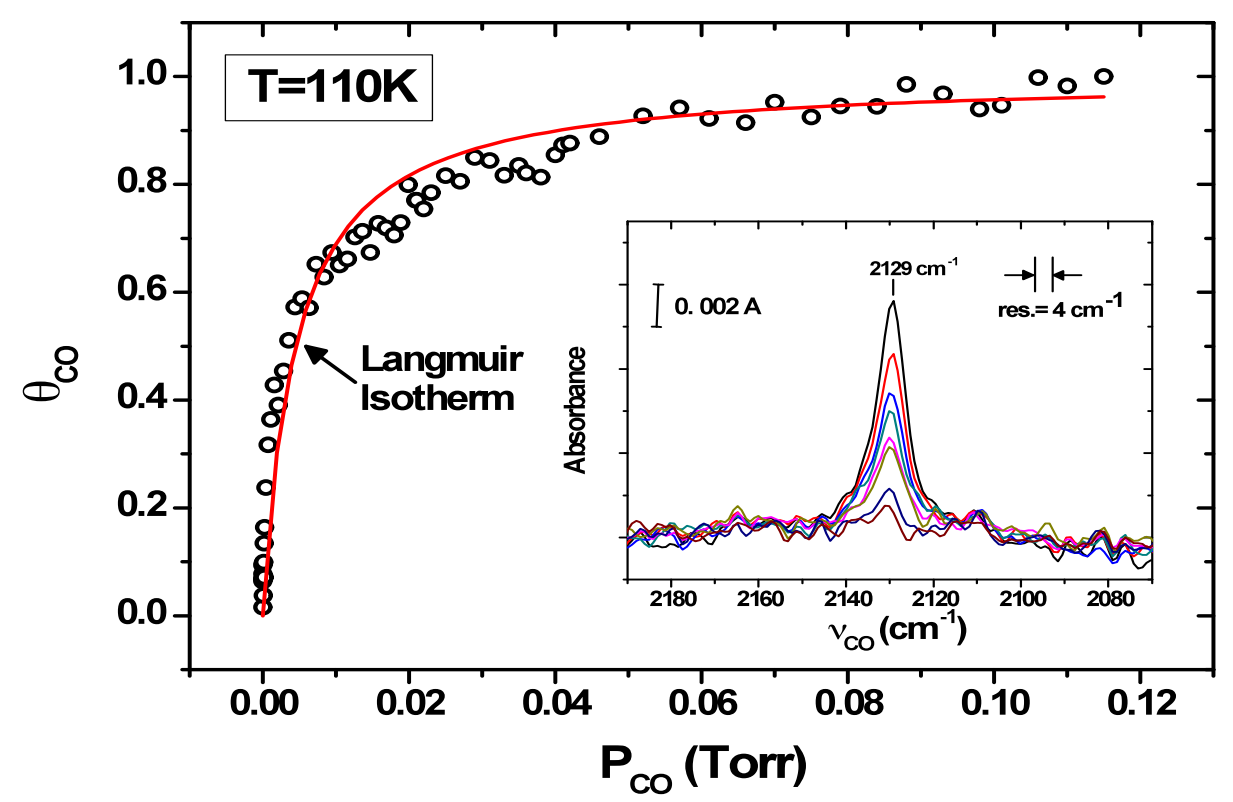

Figure 8.2. Langmuir isotherm of $\mathrm{CO}$ adsorption on $\mathrm{C}_{60}$ at $110 \mathrm{~K}$. The inserted panel shows the beginning spectral development of $\mathrm{CO}$ stretching mode.

Using the integrated infrared absorbance as a measure of equilibrium surface coverage, a set of partial isotherms were measured at temperatures in the range $82 \mathrm{~K}-130 \mathrm{~K}$, as shown in Figure 8.3a. By observing the coverage, $\theta_{\mathrm{CO}}$, at 0.001 Torr $\mathrm{CO}$ equilibrium pressure, an isobaric enthalpy of adsorption was measured and was found to be $-0.109 \pm 0.004 \mathrm{eV}$, as shown in Figure $8.3 \mathrm{~b}$. This is in good agreement with theoretical calculations of the enthalpy of adsorption of $\mathrm{CO}$ on $\mathrm{C}_{60}$ 's fcc (111) plane where an energy of adsorption of $-0.13 \mathrm{eV}$ was calculated[9]. Jiang et al. also compared the $\mathrm{CO}$ and $\mathrm{N}_{2}$ physical adsorption energies and the adsorption sites on the $\mathrm{C}_{60}$ surface [17]. The linearity of the data in Figure $8.3 \mathrm{~b}$ is consistent with little $\mathrm{CO}-\mathrm{CO}$ interaction over the range of coverage studied here. 

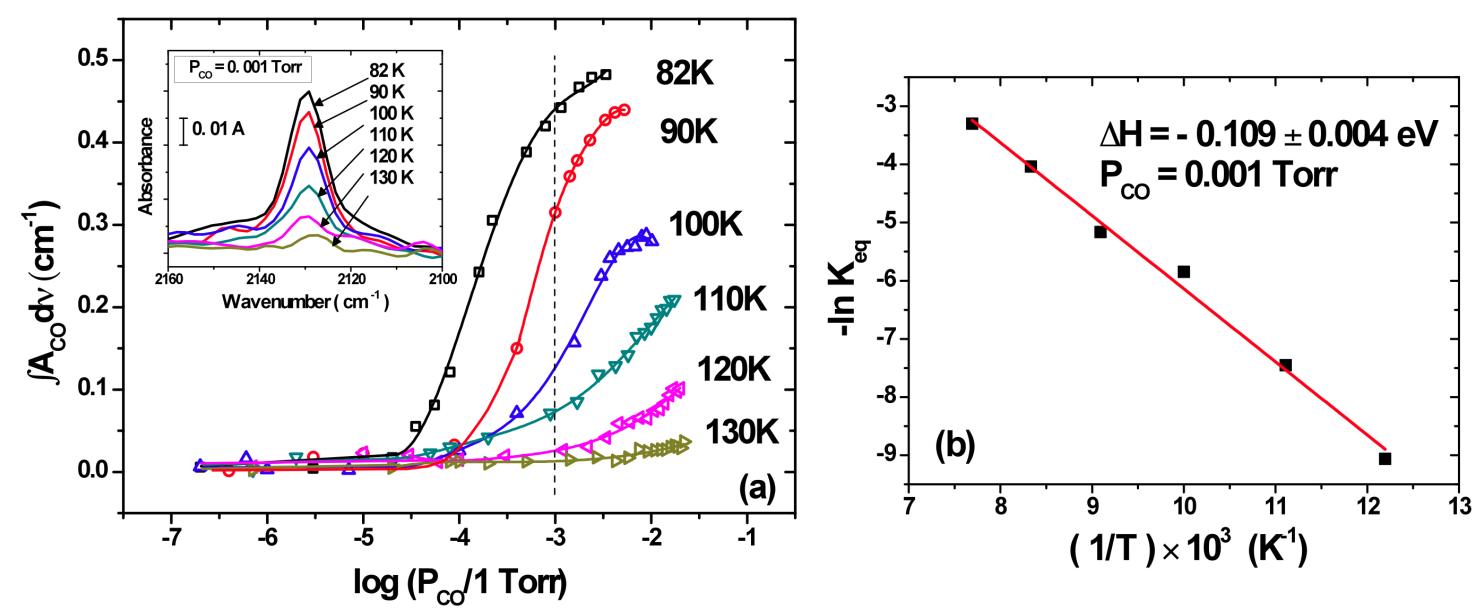

Figure 8.3. (a) Partial Langmuir isotherms of $\mathrm{CO}$ adsorption on $\mathrm{C}_{60}$ at $82-130 \mathrm{~K}$. The inserted panel shows the correspondent spectra of the CO stretching mode. (b) The determination of isobaric enthalpy of $\mathrm{CO}$ adsorption at $\mathrm{P}_{\mathrm{CO}}=0.001$ Torr.

\subsubsection{Displacement of Physisorbed $\mathrm{CO}$ by $\mathrm{He}, \mathrm{Ne}$ and Ar.}

We observed the monotonic displacement of physisorbed $\mathrm{CO}$ by $\mathrm{He}, \mathrm{Ne}$ and $\mathrm{Ar}$, and these data, obtained at $110 \mathrm{~K}$ under equilibrium conditions with a constant $\mathrm{P}_{\mathrm{CO}}=0.01$ Torr, are shown in Figure 8.4. The displacement process is plotted against the calculated flux, $F_{x}=\frac{P_{x}}{\left(2 \pi m_{x} k_{B} T\right)^{1 / 2}}$, of each added gas, assuming a gas temperature equal to that of the walls of the infrared cell, $300 \mathrm{~K}$. As the displacing gas flux increases, additional CO desorption occurs, disturbing the equilibrium and producing a decrease in the equilibrium value of $\theta_{\mathrm{co}}$. One notes that as the mass and the polarizability of the displacing gas molecule increases (polarizability of $\mathrm{He}=0.208 \AA^{3}$; $\mathrm{Ne}=0.381 \AA^{3} ; \mathrm{Ar}=1.664 \AA^{3}$ [18]) the degree of displacement of equilibrium adsorbed $\mathrm{CO}$ at a given $F_{x}$ decreases. This means that two effects may operate together in governing the displacement efficiency: (1). The van der Waals forces involved in the adsorption of the displacing molecule on $\mathrm{C}_{60}$ and governed by polarizability operate to diminish the efficiency of the $\mathrm{CO}$ displacement process, probably through interaction of the 
displacing molecule with the $C_{60}$ substrate leading to energy transfer to the $C_{60}$ substrate; (2). The mass of the displacing molecule will also govern the efficiency of energy transfer to the $\mathrm{C}_{60}$ with more massive molecules exchanging more kinetic energy with the $\mathrm{C}_{60}$ substrate, and hence exchanging less energy with the physisorbed $\mathrm{CO}$ molecules, causing a reduction in $\mathrm{CO}$ displacement efficiency.

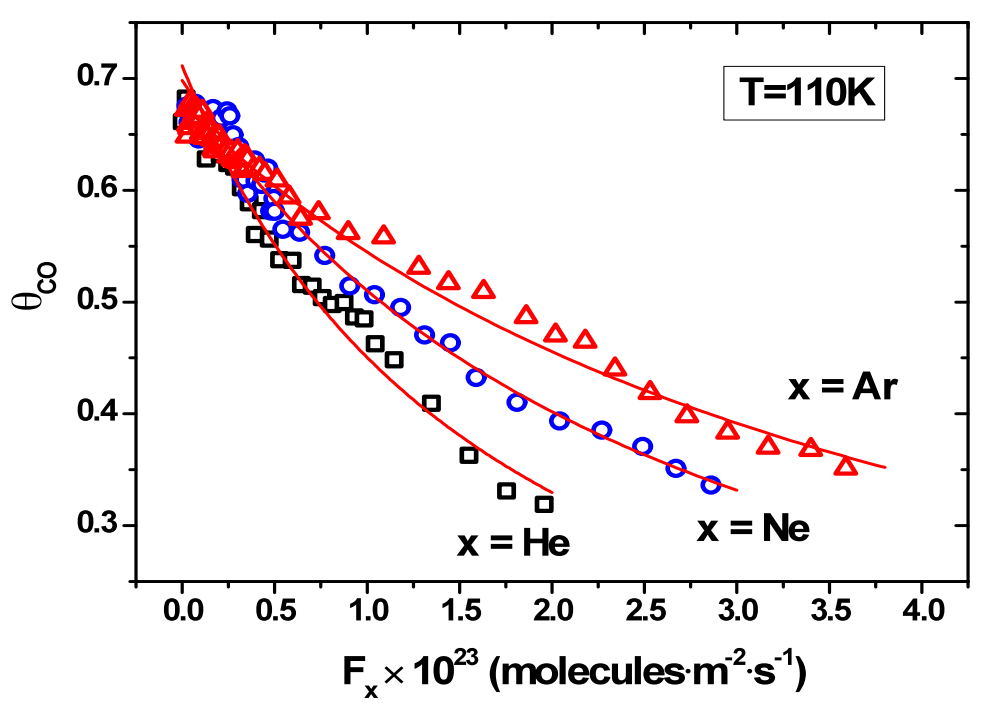

Figure 8.4. $\mathrm{CO}$ dynamic displacement by $\mathrm{He}, \mathrm{Ne}$ and $\mathrm{Ar}$. The curves are drawn in accordance with the best linear fits in Figure 8.5.

We postulate that the equilibrium between $\mathrm{CO}(\mathrm{g})$ and $\mathrm{CO}(\mathrm{a})$ is disturbed by the arrival of a displacing gas molecule in accordance with the Langmuir equations below:

$$
k_{a, C O} P_{C O}\left(1-\theta_{C O}\right)=k_{d, C O} \theta_{C O}+F_{x} \cdot \alpha_{x} \cdot \theta_{C O}
$$

where $\mathrm{k}_{\mathrm{a}}$ is the adsorption rate constant of $\mathrm{CO}(\mathrm{g})$ and $\mathrm{k}_{\mathrm{d}}$ is the desorption rate constant of $\mathrm{CO}(\mathrm{a})$. The gas flux of the displacing molecule is $F_{x}$, and $\alpha_{\mathrm{x}}$ is the displacement efficiency. Therefore, 


$$
\begin{gathered}
\frac{1}{\theta_{C O}}=1+\frac{k_{d, C O}}{k_{a, C O} P_{C O}}+\frac{\alpha_{x}}{k_{a, C O} P_{C O}} \cdot F_{x} \\
\frac{d\left(1 / \theta_{C O}\right)}{d F_{x}}=\frac{\alpha_{x}}{k_{a, C O} P_{C O}}
\end{gathered}
$$

Using the linearized form of the Langmuir isotherm (eqn. 2), Figure 8.5 shows the relative efficiency of displacement of $\mathrm{CO}$ by the three molecules at $110 \mathrm{~K}$. Setting the relative efficiency for $\mathrm{CO}$ displacement by $\mathrm{He}, \alpha_{H e}^{r e l}=1$, it is seen that for $\mathrm{Ne}$ and $\mathrm{Ar}$, the relative displacement efficiency decreases to $\alpha_{\mathrm{Ne}}^{r e l}=0.64$ and $\alpha_{A r}^{r e l}=0.44$ at $110 \mathrm{~K}$ in accordance with their increasing polarizabilities and masses.

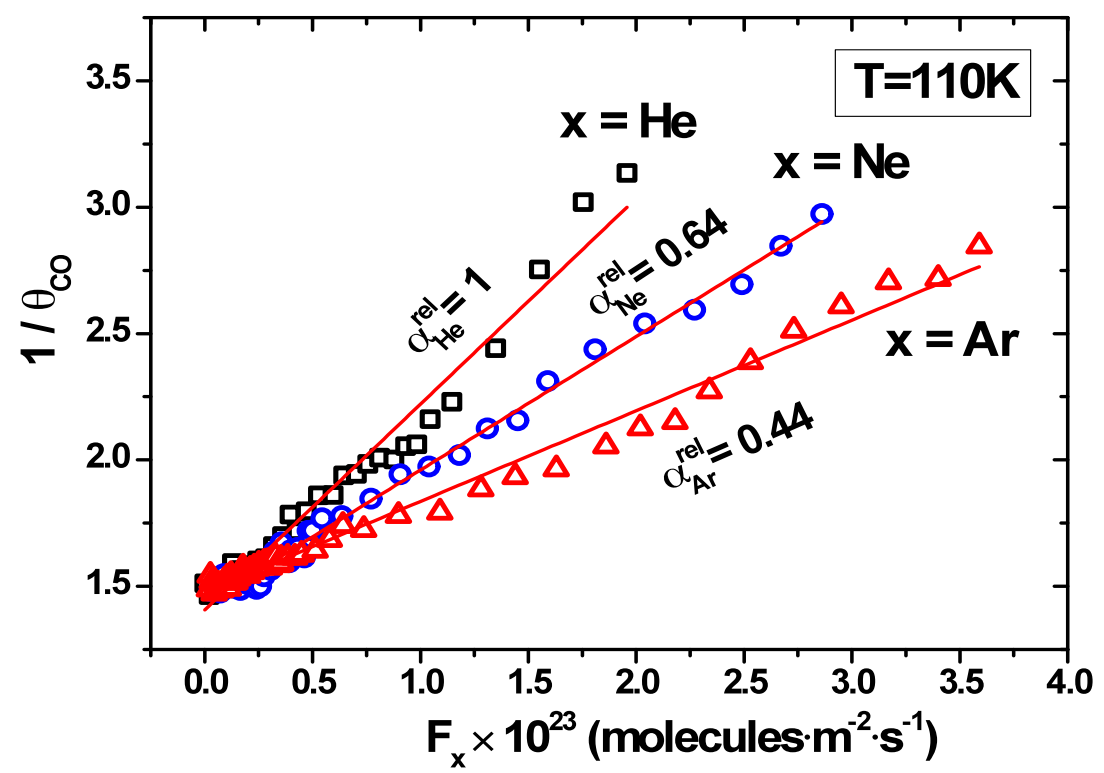

Figure 8.5. Linearized isotherms of $\mathrm{CO}$ dynamic displacement by $\mathrm{He}, \mathrm{Ne}$ and $\mathrm{Ar}$, and their relative efficiency. 


\subsubsection{Absolute Rate of Dynamic Displacement}

A separate experiment was performed in which the approximate rate of $\mathrm{CO}$ displacement during the attainment of equilibrium was measured at $110 \mathrm{~K}$ when a small pulse of $\mathrm{He}$ was added, as shown in Figure 8.6. This experiment indicated that for a fluence of $\mathrm{He}=3 \times 10^{24}$ molecules $\mathrm{m}^{-2}$, the coverage of $\mathrm{CO}$ decreased by $\sim 0.67 \mathrm{ML} \cong 4 \times 10^{20}$ molecules $\cdot \mathrm{m}^{-2}$ during the measured short time period of the displacement process $(\sim 10 \mathrm{~s})$ as shown in Figure 8.6. Thus the efficiency of the $\mathrm{CO}$ displacement process during the disturbance of the $\mathrm{CO}$ equilibrium condition is about $\sim 1 \mathrm{CO}$ displacement event for $\sim 10^{4} \mathrm{He}$ collisions. The displacement efficiencies, per collision, of the other molecules, $\mathrm{Ne}$ and $\mathrm{Ar}$, scale as the measured relative values of $\alpha_{N e}^{r e l}$ and $\alpha_{A r}^{r e l}$.

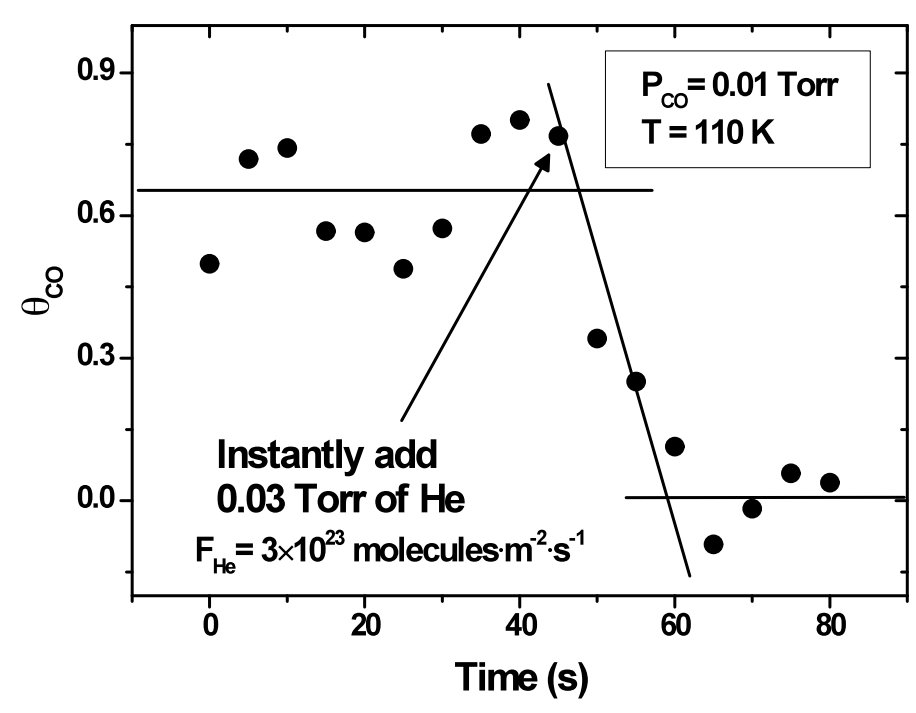

Figure 8.6. Absolute rate measurement of $\mathrm{CO}$ dynamic displacement by $\mathrm{He}$.

\subsubsection{Displacement of Physisorbed CO by Kr}

Figure 8.7 shows the experimental data for $\mathrm{Kr}$ as a displacing molecule, compared to $\mathrm{He}, \mathrm{Ne}$ and $\mathrm{Ar}$. Here it is seen that $\mathrm{Kr}$ behaves completely differently from the lighter noble gases, causing very efficient displacement as 
may be judged from the steep slope for $\mathrm{Kr}$ in Figure 8.7. In contrast to $\mathrm{He}, \mathrm{Ne}$ and $\mathrm{Ar}$, the adsorption energy of $\mathrm{Kr}$ on $\mathrm{C}_{60}(-0.165 \mathrm{eV}$ [10]) exceeds that of $\mathrm{CO}$ on $\mathrm{C}_{60}$ where $\Delta \mathrm{H}_{\mathrm{CO}}=-0.109 \mathrm{eV}$ as shown in Figure $8.3 \mathrm{~b}$. Thus, $\mathrm{Kr}$ will efficiently displace $\mathrm{CO}$ from $\mathrm{C}_{60}$ because of its higher adsorption energy, just has been found for similar displacement processes from the adsorption sites on single walled nanotubes $[19,20]$ which are strongly driven by the exothermicity of the adsorbate displacement process.

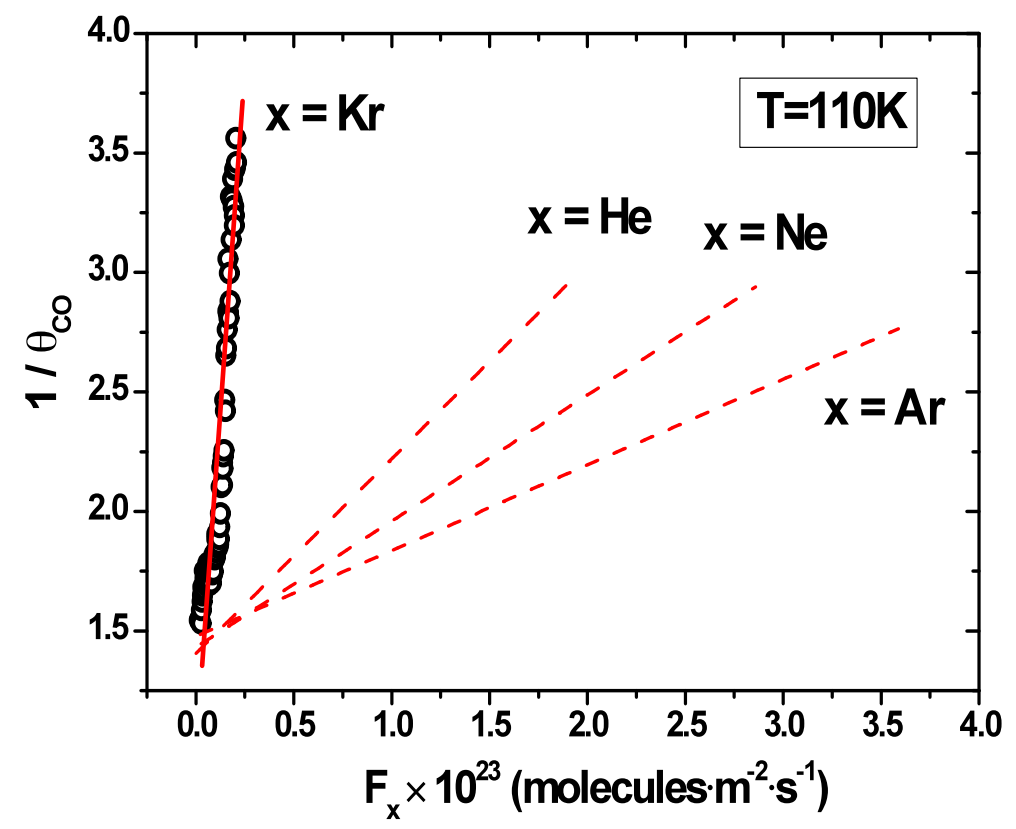

Figure 8.7. Linearized isotherms of $\mathrm{CO}$ exothermic replacement by $\mathrm{Kr}$. The lines for $\mathrm{He}$, $\mathrm{Ne}$ and $\mathrm{Ar}$ are shown for comparison purpose.

\subsubsection{Displacement of Physisorbed $\mathrm{CO}$ by $\mathrm{H}_{2}$.}

Figure 8.8 shows the Langmuirian plot for $\mathrm{H}_{2}$ as a displacing gas. It may be seen that $\mathrm{H}_{2}$ rivals $\mathrm{He}$ in $\mathrm{CO}$ displacement efficiency under equilibrium conditions, exhibiting $\alpha_{\mathrm{H}_{2}}^{r e l}=0.87$. This rapid rate of dynamical displacement of $\mathrm{CO}$ by $\mathrm{H}_{2}$ is inconsistent with its high polarizability $\left(\mathrm{H}_{2}=0.787 \AA^{3}\right.$ [18]) compared to $\mathrm{He}, \mathrm{Ne}$ and Ar. A high polarizability would result in increased life time and energy 
exchange with the $\mathrm{C}_{60}$ substrate, and hence produces a lower $\mathrm{CO}$ displacement efficiency, which is not observed. However, because of its low mass, $\mathrm{H}_{2}$ would be expected to lose less energy upon collision with the $\mathrm{C}_{60}$ substrate, leading to the opposite effect, enhanced $\mathrm{CO}$ displacement, when considered solely from a momentum transfer point of view. Furthermore, at $300 \mathrm{~K}$, the rotational partition function for $\mathrm{H}_{2}$ has developed almost to its maximum meaning that in addition to $3 / 2 \mathrm{k}_{B} T$ translational energy, $\mathrm{H}_{2}$ also carries about $\mathrm{k}_{\mathrm{B}} \mathrm{T}$ of rotational energy, a portion of which could be utilized in causing $\mathrm{CO}$ displacement, and leading to enhanced $\mathrm{CO}$ dynamical displacement by $\mathrm{H}_{2}$.

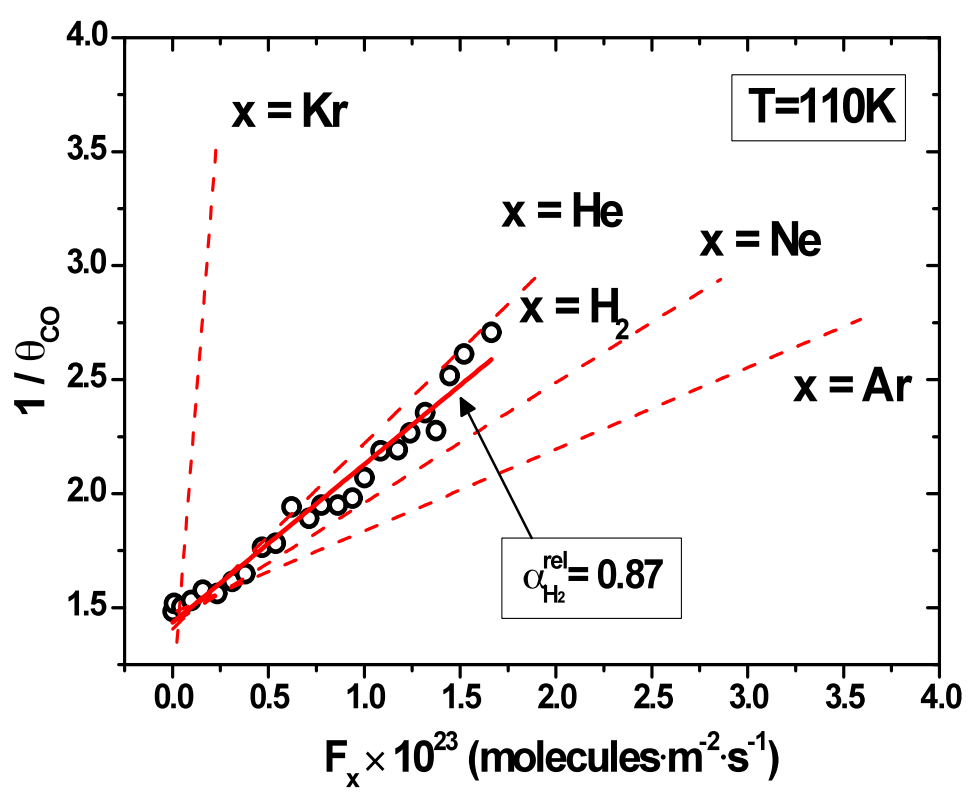

Figure 8.8. Linearized isotherms of $\mathrm{CO}$ displacement by $\mathrm{H}_{2}$, and its relative efficiency $\alpha_{\mathrm{H}_{2}}^{\text {rel }}=0.87$. The lines for $\mathrm{He}, \mathrm{Ne}, \mathrm{Ar}$ and $\mathrm{Kr}$ are shown for comparison purpose.

\subsubsection{Temperature Dependence of Physisorbed CO Displacement by Ar.}

The adsorption energy of $\mathrm{CO}$ and $\mathrm{Ar}$ are closely similar to each other on $\mathrm{C}_{60}$. For $\mathrm{Ar}$, the theoretical adsorption energy is $-0.130 \mathrm{eV}$ at zero coverage [10], whereas for $\mathrm{CO}$ we measure an energy of $-0.109 \mathrm{eV}$ at intermediate coverage as 
shown in Figure 8.3b. Thus, Ar exposures will tend to form an adsorbed Ar layer of finite coverage, and produce $\mathrm{CO}$ displacement by a rapid process involving the competitive physical adsorption of Ar with $\mathrm{CO}$. As this Ar layer develops, and repulsive $\mathrm{Ar}-\mathrm{Ar}$ and $\mathrm{Ar}-\mathrm{CO}$ forces build up, the adsorption energy of Ar will drop below that of $\mathrm{CO}$ and a transfer from an exothermic $\mathrm{CO}$ replacement mechanism to a CO dynamical displacement mechanism will take place. To test this hypothesis, we performed Ar-induced displacement experiments at different $\mathrm{C}_{60}$ temperatures, where higher and higher Ar coverages would be expected at the lower temperatures. As shown in Figure 8.9, as the temperature is lowered from $110 \mathrm{~K}$ to $90 \mathrm{~K}$, the displacement of $\mathrm{CO}$ becomes more efficient, as judged by the increase in initial slope of the $1 / \theta_{\mathrm{CO}}$ curves. In addition, for the Ar displacement experiments at $100 \mathrm{~K}$ and $90 \mathrm{~K}$, a transition from the exothermic $\mathrm{CO}$ replacement region to the $\mathrm{CO}$ dynamical displacement region of lower slope is observed at the circled breakpoint. Depending upon temperature, the Ar molecule can act either in the site competition mode, where simple exothermic $\mathrm{CO}$ replacement occurs from an Ar layer of finite coverage when the Ar adsorption energy exceeds that of $\mathrm{CO}$, or the Ar molecule can behave dynamically at higher Ar coverages where $\mathrm{V}_{\mathrm{CO}}$ is larger than $\mathrm{V}_{\mathrm{Ar}}$ (see Figure 8.10). Dynamical behavior occurs above a certain critical Ar flux and Ar coverage. At Ar fluxes below the break point, exothermic $\mathrm{CO}$ site replacement by Ar occurs, because its adsorption energy exceeds that of $\mathrm{CO}$ in this region of $\mathrm{Ar}$ coverage. At Ar fluxes above the breakpoint, the $\mathrm{Ar}$ adsorption energy has fallen due to repulsive $\mathrm{Ar}-\mathrm{Ar}$ and possibly Ar-CO interactions, and the Ar begins to behave as a dynamical particle where a slower dynamical displacement process dominates, causing the slope of the displacement curve to diminish. At $110 \mathrm{~K}$, the dynamical process dominates at all $F_{A r}$, and the breakpoint is not visible. 


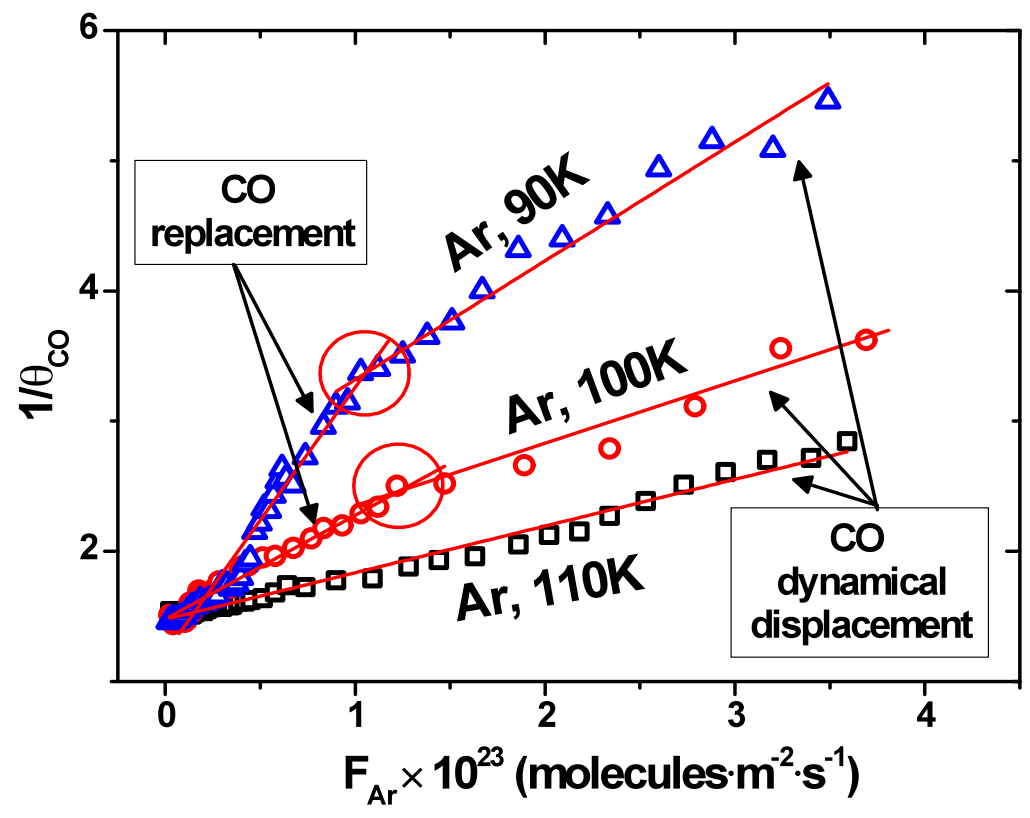

Figure 8.9. Temperature dependence of $\mathrm{CO}$ displacement by $\mathrm{Ar}$ at 90, 100 and $110 \mathrm{~K}$. The breakpoints in the circled region show the transition from exothermic $\mathrm{CO}$ replacement to CO dynamical displacement.

Figure 8.10 schematically shows a pair of adsorption potential wells for $\mathrm{CO}$ and for the displacing noble gases. The adsorption potential, $\mathrm{V}_{\mathrm{x}}$, for $\mathrm{He}$ and $\mathrm{Ne}$ is always smaller than $\mathrm{V}_{\mathrm{CO}}$ and in this situation dynamical $\mathrm{CO}$ displacement occurs exclusively, as observed. For $\mathrm{Kr}$, the adsorption potential is always larger than $\mathrm{V}_{\mathrm{CO}}$, and an exothermic CO replacement process therefore occurs efficiently at all $\mathrm{Kr}$ fluxes. In the right hand side of Figure 8.10, the adsorption energy of $\mathrm{Ar}$ initially exceeds that of $\mathrm{CO}$ at low $\mathrm{Ar}$ coverages and an exothermic $\mathrm{CO}$ replacement process occurs. As the Ar coverage increases, and Ar-Ar and Ar-CO repulsive forces increase causing the Ar adsorption energy to fall below that of $\mathrm{CO}$, the exothermic $\mathrm{CO}$ replacement process reverts to a dynamical type of $\mathrm{CO}$ displacement process as the adsorption of additional Ar ceases to occur. 

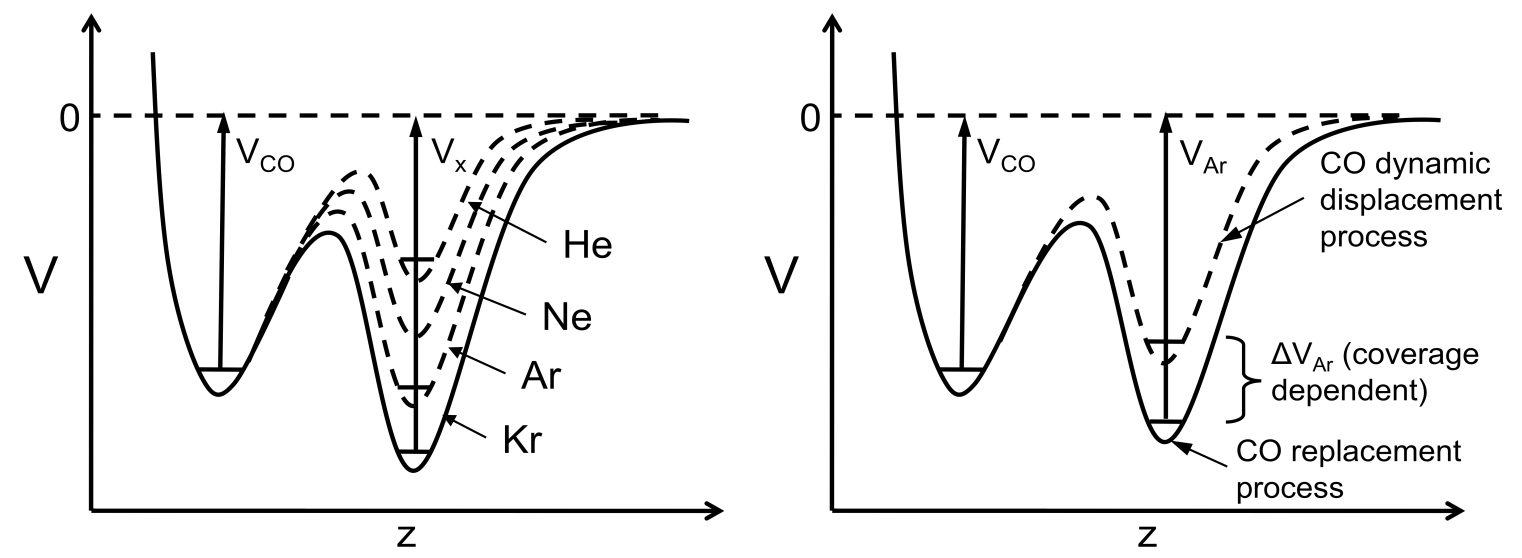

Figure 8.10. (a) A pair of adsorption potential wells for $\mathrm{CO}$ and displacing noble gases. (b) The $\mathrm{CO}$ adsorption potential well and comparison with the coverage dependent Ar adsorption potential well.
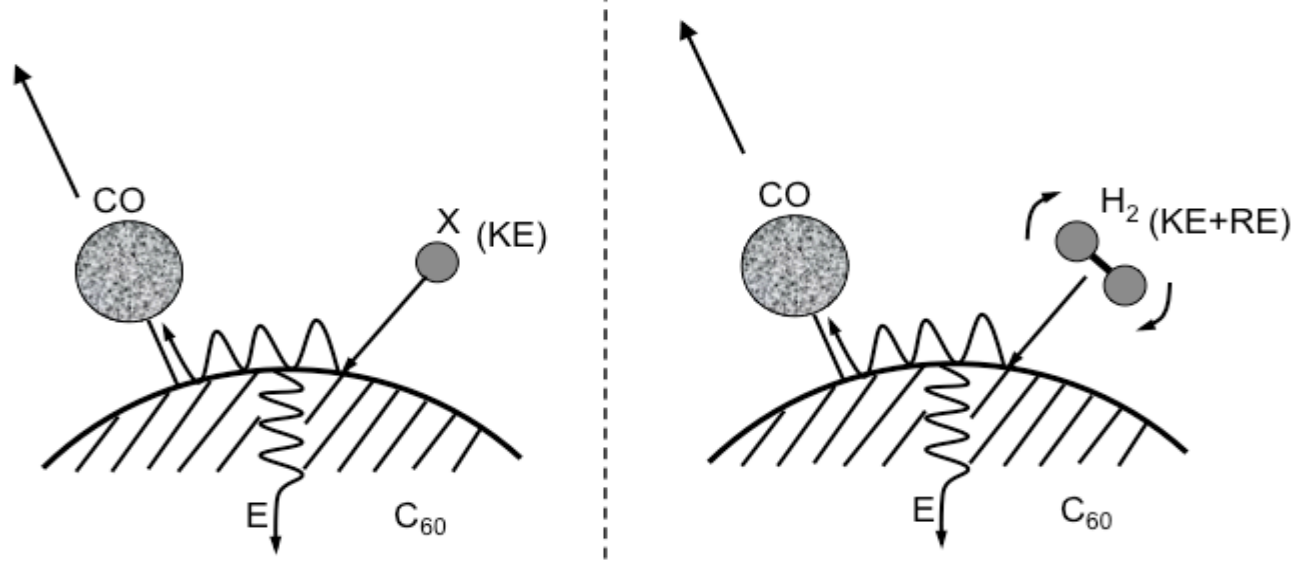

Figure 8.11. (a) A schematic picture of $\mathrm{CO}$ dynamic displacement by single-atom noble gases with kinetic energy. (b) $\mathrm{CO}$ dynamic displacement by diatom $\mathrm{H}_{2}$ molecules with kinetic and rotational energy. 


\subsection{Summary}

In summary, we observed that physisorbed $\mathrm{CO}$ molecules can be displaced by another molecule, either in dynamic displacement process or exothermic replacement, depending on their adsorption energies, as shown in Figure 8.11. The incoming molecule could change its displacement mechanism from one to another depending on temperature and coverage. Rotational energy of the incoming molecule may also be involved in supplying energy for the displacement process.

\subsection{Reference:}

1. Bottani, E.J. and J.M.D. Tascón, Adsorption by Carbons, 1st Edition, 2008: Elsevier Science.

2. Levine, I.N., Physical Chemistry, 6th edition, 2008: McGraw-Hill Science/Engineering/Math.

3. Adamson, A.W. and A.P. Gast, Physical Chemistry of Surfaces, 6th Edition, 1997, New York: Wiley-Interscience.

4. Brunauer, S., P.H. Emmett, and E. Teller, Adsorption of Gases in Multimolecular Layers (BET). J. Am. Chem. Soc., 1938. 60(2): p. 309.

5. Yates, J.T. and D.W. Goodman, Carbon monoxide chemisorption on $\mathrm{Ni}(100)-$ direct detection of adsorbate-adsorbate interactions by desorption kinetic measurements. J. Chem. Phys., 1980. 73: p. 5371.

6. Takagi, N., J. Yoshinobu, and M. Kawai, Temporal and Local Reduction of a Potential Energy Well under Dynamic Equilibrium: CO on Ni(100). Phys. Rev. Lett., 1994. 73(2): p. 292.

7. Holleman, I., et al., An infrared study on CO intercalated in solid C60. J. Chem. Phys., 1999. 110: p. 2129. 
8. Fastow, M., et al., IR Spectra of CO and NO Adsorbed on C60. J. Phys. Chem. , 1992. 96: p. 6126.

9. Folman, M., M. Fastow, and Y. Kozirovski, Surface Heterogeneity of C60 As Studied by Infrared Spectroscopy of Adsorbed CO and Adsorption Potential Calculations. Langmuir, 1997. 13: p. 1118.

10. Trasca, R.A., et al., Gas adsorption on a C60 monolayer. Phys. Rev. E, 2008. 77: p. 041603.

11. Basu, P., T.H. Ballinger, and J.T. Yates, Wide temperature range IR spectroscopy cell for studies of adsorption and desorption on high area solids.

Rev. Sci. Instrum., 1988. 59: p. 1321.

12. Martin, M.C., et al., Observation and assignment of silent and high-order vibrations in the infrared transmission of C60 crystals. Phys. Rev. B, 1994. 50(1): p. 173.

13. Wang, K.-A., et al., Observation of higher-order infrared modes in solid C60 films. Phys. Rev. B, 1993. 48: p. 11375.

14. Iglesias-Groth, S., F. Cataldo, and A. Manchado, Infrared spectroscopy and integrated molar absorptivity of $\mathrm{C} 60$ and $\mathrm{C} 70$ fullerenes at extreme temperatures. Mon. Not. R. Astron. Soc., 2011. 413: p. 213.

15. Kondratieva, E.V., O.V. Manoilova, and A.A. Tsyganenko, Integrated Absorption Coefficient of Adsorbed CO. Kinetics and Catalysis, 2008. 49(3): p. 451.

16. Kim, K., The integrated intensity of the carbon monoxide fundamental band. J. Quant. Spectrosc. Radiat. Transfer, 1983. 30: p. 413.

17. Jiang, J., J.B. Klauda, and S.I. Sandler, Hierarchical Modeling N2 Adsorption on the Surface of and within a C60 Crystal: From Quantum Mechanics to Molecular Simulation. J. Phys. Chem. B, 2005. 109: p. 4731.

18. NIST Computational Chemistry Comparison and Benchmark Database.

19. Kondratyuk, P. and J.T. Yates., Desorption kinetic detection of different adsorption sites on opened carbon single walled nanotubes: The adsorption of $n$ nonane and CCl4. Chemical Physics Letters, 2005. 410: p. 324.

20. Kondratyuk, P. and J.T. Yates, Molecular Views of Physical Adsorption Inside and Outside of Single-Wall Carbon Nanotubes. Acc. Chem. Res., 2007. 40: p. 995. 


\section{Appendix II: Experimental Details}

\subsection{Temperature Control}

The temperature control is crucial in the experiments. In our system, we measure the temperature using a K-type thermocouple, which is made of two thin (diameter $=0.005 ")$ metal wires - one chromel and one Alumel. The two thin wires are welded together using a spot welder. One should be careful when welding the thermocouple wires, using appropriate voltage. Too low voltage may cause the joint to loosen, and too high voltage can melt and break the wires. The thermocouple is connected to a thermocouple compensator, and then to a millivolt amplifier. The temperature output can be read by a computer using a Labview temperature control program.

The relative accuracy of temperature reading is $\pm 0.1 \mathrm{~K}$, while the absolute accuracy is about $\pm 2 \mathrm{~K}$. The temperature reading is affected by the battery voltage in the thermocouple compensator. A new battery will show higher temperature reading. If a continuous temperature decreasing under the same experimental condition (for example, cooling the dewar only with liquid $N_{2}$ ) is observed, one should consider replacing the battery.

The lowest sample temperature that can be reached is about $84 \mathrm{~K}$ with liquid $\mathrm{N}_{2}$ cooling only. Further cooling for about $10 \mathrm{~K}$ can be achieved by bubbling $\mathrm{He}$ through the I- $\mathrm{N}_{2}$ [1]. However this is a tricky technique in the actual experiments.

First of all, the tip of the He tube should be placed close to the bottom of the dewar. The end of the dewar has a smaller diameter and hence the space is limited. It may take a while to find the right place to insert the tube toward the end. This will make the He cooling more efficient and more stable. 
Though the tip of the He tube is placed close to the end of the dewar, it should not touch the bottom. This is because some ice may fall into the dewar and could then block the He gas flow. One should lift the He tube up for about 1 $\mathrm{cm}$ from the bottom and fix the tube at that position. This will help to prevent the blocking of He flow yet keep the cooling efficiency. He gas of $99.95 \%$ purity from Praxair is used. Sometimes, if the He gas is not very pure (containing water), it will form ice inside the He tube and finally block the gas flow. One may increase the gas flow rate to blow out the ice, or lift the He tube out from the dewar and let it warm up. However, both ways will affect the temperature stability of the sample.

By controlling the He flow rate, we can roughly control the cooling efficiency. Usually the He flow pressure is less than $5 \mathrm{psi}$, and the lowest temperature should reach about $76 \mathrm{~K}$. Increasing the He flow rate can further cool the dewar to about $72 \mathrm{~K}$, however the temperature will not be as stable as that with lower flow rates. Adding liquid $\mathrm{N}_{2}$ into the dewar will affect the temperature stability slightly, as the I- $\mathrm{N}_{2}$ temperature is higher than the He-cooled dewar. It may cause the temperature to shift within a range of $0.5 \mathrm{~K}$.

During a long experiment, as $\mathrm{He}$ is used up, the He flow pressure will slowly drop and therefore cause an increase of temperature. One may slightly increase the He flow rate to compensate the decreasing of He pressure, but it should not be performed until necessary because it often causes unpredictable shift of stable temperature.

\subsection{Sample Preparation}

The sample $\left(\mathrm{KBr}\right.$ or $\left.\mathrm{SiO}_{2}\right)$ is pressed on a $\sim 1.5 "-2$ " sized tungsten grid using a stainless steel jig with a $7 \mathrm{~mm}$ diameter die for compression. To clean the grid, one may put the grid in a small beaker and fill the beaker with water or acetone. To clean the $\mathrm{SiO}_{2}$ sample on the grid, $1 \mathrm{M} \mathrm{NaOH}$ solution may be used. Sonicate 
the beaker with grid for 5-10 minutes, and then rinse the grid with acetone for 2-3 times.

The $\mathrm{KBr}$ sample is prepared using finely ground $\mathrm{KBr}$ powder $(99.0 \%$, Mallinckrodt). The $\mathrm{KBr}$ powder is heated to $100^{\circ} \mathrm{C}$ in a vacuum oven for 2 hours to remove absorbed water. To press the $\mathrm{KBr}$ sample, we use a hydraulic laboratory press with 3000 psi pressure. Too much pressure may cause cracks on the sample. To press the $\mathrm{SiO}_{2}$ sample, we use aerosil 200 (amorphous $\mathrm{SiO}_{2}$ powder) with a pressure of 5000 psi.

The sample prepared in this way usually contains some adsorbed water and organic species, which can be seen from IR absorption bands at $\sim 3200 \mathrm{~cm}^{-1}$ and $\sim 2800 \mathrm{~cm}^{-1}$. To remove these contaminants, we can heat the sample in vacuum to high temperature for a few minutes. For $\mathrm{SiO}_{2}$ sample, one may need to heat it to $1000 \mathrm{~K}$ to remove all adsorbed water (associated water with surface $\mathrm{OH}$ groups). However, for $\mathrm{KBr}$ sample, the heating temperature may not exceed $800 \mathrm{~K}$ as $\mathrm{KBr}$ may sublime and fall off from the grid.

For unknown reasons, the contact between the sample holder ( $\mathrm{Ni}$ bars) and the W grid may leak current when using the electronic heating. It significantly reduces the heating efficiency for the sample, and the highest temperature with maximum power input is only about $400 \mathrm{~K}$. This problem can be solved by adding a pair of gold or copper foils at the contact points between the Ni bars and the $\mathrm{W}$ grid.

\subsection{Lyman- $\alpha$ Lamp Operation}

The Lyman- $\alpha$ lamp is a hydrogen discharge lamp, operated with $10 \% \mathrm{H}_{2}$ / $90 \% \operatorname{Ar}$ mixed gas. The premixed gas is obtained by specified order from GT\&S Inc. The design of lamp is shown in Figure 1.3. The pressure during gas flow is $1.000 \pm 0.010$ Torr. To accurately maintain a stable pressure, all valves and 
regulators along the $\mathrm{H}_{2}$ gas line have been set to an optimum position. One should only open and close the valve of the gas cylinder when operating the lamp, avoid changing any other valve position. The pressure of $\mathrm{H}_{2}$ gas flow should automatically reach $1.000 \pm 0.010$ Torr. When the lamp is turned on, the gas pressure may increase by about 0.01 Torr. If the gas pressure shifts a lot from 1.000 Torr, one may slightly adjust the regulator attached to the gas cylinder. It could take a few minutes for the pressure to reach equilibrium, therefore any change should be performed slowly.

The microwave discharge power supply has two switches. Before starting the lamp, we should turn on the white switch first for at least 5 mins to let the power supply warm up. It is noted that a longer warm-up time may increase the power stability. Therefore, a half hour warm-up time is suggested. Also, be sure to turn on the cooling gas flow before starting the lamp. To start the lamp, we turn on the red switch, increase the power to 20-30 $\mathrm{W}$, and use the tesla coil to ignite the lamp. In some cases, the lamp will start automatically, possibly due to an excitation by a cosmic ray. During the year of 2013 when the solar activity cycle reaches a maximum, the lamp can always start itself. After starting the lamp, we increase the power to the normal operating power $(60 \mathrm{~W})$. The power may be increased in some cases when a higher photon flux is needed, however it should not exceed $80 \mathrm{~W}$. Sometimes, the power output may fluctuate, or slowly shift from the set power. One should keep monitoring the power output during experiments and adjust it when needed.

One may notice that sometimes the lamp shows a pale color. It is usually due to an exposure of lamp with air, and it should come to the regular color in a few minutes. Sometimes, the lamp may flash, which may be due to a misplacement of the discharge clamp. It can be corrected by adjusting the screws on the clamp, until the lamp becomes stable. 
The lamp flux is calibrated by a chemical actinometer method using $\mathrm{N}_{2} \mathrm{O}$ gas [2]. Briefly, 10 Torr $\mathrm{N}_{2} \mathrm{O}$ gas is introduced into the cell and is photolyzed by Lyman- $\alpha$ radiation at normal working condition. The destruction rate of $\mathrm{N}_{2} \mathrm{O}$ gas is measured by IR integrated absorbance. The lamp flux can be calculated from the measured $\mathrm{N}_{2} \mathrm{O}$ photo-destruction rate and the known destruction efficiency (photons $/ \mathrm{N}_{2} \mathrm{O}$ ). The total flux of this lamp at normal operating condition is $(1.28 \pm 0.36) \times 10^{15}$ photons $\mathrm{cm}^{-2} \mathrm{~s}^{-1}$.

\subsection{Infrared Spectroscopy Operation}

Infrared spectrometer operation is a major part in our measurement. The whole beam line of the spectrometer system is purged by dry and $\mathrm{CO}_{2}$-free air generated from a FTIR purge gas generator supplied by Parker Balston.

A liquid $\mathrm{N}_{2}$ cooled MCT detector is used to record the infrared spectra instead of the build-in detector to increase the sensitivity and signal-to-noise ratio. The detector has a vacuum dewar to hold liquid $\mathrm{N}_{2}$. However, the seal of vacuum is not so good and evacuation of detector should be performed regularly. Usually, a continuous growth of condensed water band in the IR spectra indicates the detector need to be pumped. One may pump the detector using a special adaptor according to the procedures on the Bruker Tensor spectrometer user's manual. After pumping the detector, the beam path should be realigned to ensure a high sensitivity. The strength of infrared beam received by the detector is measured by "ADC count" in the OPUS program. The beam line can be calibrated by adjusting the screws behind the mirrors in each reflecting box, to gain a signal count as high as possible. The ideal infrared signal count should be above 25000. One may need to adjust each mirror several times in order to reach the desired sensitivity.

The spectral data processing is an important and time-consuming part in our data analysis. Several frequently used processing methods include spectra 
subtraction, integration of a peak area, saving the data into an ASCIl file. The OPUS program lacks some functions causing one to manually analyze spectra one by one, copy or write down the results and paste into another analysis program such as Excel or Origin. To simply the data processing and reduce the repetitive work, I wrote several program codes that can be implemented into the macros of the OPUS program. These codes can save a huge amount of data processing time, completing 2 hours work in 2 seconds!

A. Auto saving - this code can save multiple spectra into data point file (.DPT) at a time.

\author{
VARIABLES SECTION \\ FILE $<$ File $>=\mathrm{AB}$; \\ STRING $<$ path $>=$ "; \\ STRING <FileName $>$ = "; \\ STRING < SampleNumber $>$ = ";
}

PROGRAM SECTION

REM This macro is used to save all selected files from OPUS format to data point files. Developed by C.Yuan

REM All selected files are stored in a single file variable as an array $<$ path $>$ = GetOpusPath ();

UserDialog ('0', STANDARD, FILE:[<File>:AB], EDIT:'<path>', BLANK, BLANK, BLANK, BLANK, BLANK, BLANK, BLANK, BLANK, BLANK, BLANK, BLANK, BLANK);

REM Process all loaded files in a loop, use the FILE Variable as loop count StartLoop ([<File>:AB], 0);

REM Within such a loop the proper file is automatically determined $<$ FileName $>=$ GetParameter $([<$ File $>$ :AB $]$, NAM);

SaveAs $\left(\left[<\right.\right.$ File $>$ :AB], $\left\{\mathrm{DAP}={ }^{\prime}<\right.$ path $>$ ', OEX='0', SAN='<FileName $>$.DPT', COF=64, INP='C: $\backslash$ OPUS $\backslash$ METHODS', IFP='C: $\backslash$ OPUS $\backslash$ METHODS', INM='DEFAULT', IFN='DEFAULT', DPA=5, DPO=5, SEP=' ', YON='0', ADP='1'\});

EndLoop (0);

B. Auto subtraction - this code can subtract baseline spectrum from multiple spectra at a time.

VARIABLES SECTION 
FILE $<$ File $>=A B$;

FILE $<$ File Subtract $>=A B$;

\title{
PROGRAM SECTION
}

REM This macro is used to subtract all selected spectrum from one spectrum automatically. Developed by C.Yuan

REM Show a User Dialog for selecting the files to be processed

REM All selected files are stored in a single file variable as an array

UserDialog ('0', STANDARD, FILE:[<File>:AB], FILE:[<File Subtract>:AB], BLANK, BLANK, BLANK, BLANK, BLANK, BLANK, BLANK, BLANK, BLANK, BLANK, BLANK, BLANK);

REM Process all loaded files in a loop, use the FILE Variable as loop count

StartLoop ([<File>:AB], 0);

REM Within such a loop the proper file is automatically determined

Subtract $([<$ File $>: A B],[<$ File Subtract $>: A B],\{S U B=3, S U N=2, S X 1=4000.000000$, $\mathrm{SX} 2=400.000000\})$;

EndLoop (0);

C. Auto integration - this code can extract peak area integration results from multiple spectra at a time and export into a .txt file.

\author{
VARIABLES SECTION \\ FILE $<$ File $>=\mathrm{AB} /$ Integ; \\ NUMERIC $<$ Row $>=1$; \\ STRING $<$ Path $>=$ "; \\ STRING <FileName $>=$ "; \\ STRING <PeakArea> = "; \\ STRING <RowName $>$ = "; \\ STRING < RowHeadName> = "; \\ NUMERIC $<$ Index $>=1$; \\ STRING $<$ PeakAreaLine $>$ = ";
}

PROGRAM SECTION

REM This macro is used to export a number of integration results to a text file. Developed by C.Yuan

$<$ Path $>$ = GetOpusPath 0 ;

REM In the userdialog, select files, enter export file name, and path

UserDialog ('0', STANDARD, FILE:[<File>:AB/Integ], EDIT:'<FileName>', EDIT:'<Path>', BLANK, BLANK, BLANK, BLANK, BLANK, BLANK, BLANK, BLANK, BLANK, BLANK, BLANK);

REM Rowname is the peak name of integration,Row is the number of peaks $<$ Row $>=$ FromReportHeader ([<File $>$ :AB/Integ $], 1,0,1$, RIGHT); 
StartLoop $(<$ Row $>, 1)$;

$<$ RowName $>=$ FromReportMatrix $([<$ File $>$ :AB/Integ $], 1,0,<$ Index $>, 1)$;

If $(<$ Index $>$, .EQ., 1$)$;

$<$ RowHeadName $>$ = 'Peak Areas of $<$ RowName $>$ ';

Else ();

$<$ RowHeadName $>$ = '<RowHeadName $>\quad$ Peak Areas of $<$ RowName $>$ ';

Endif 0;

$<$ Index $>=<$ Index $>+1$;

EndLoop (1);

REM Create a text file with a first line of peak name

TextToFile ('<Path>', '<FileName>.txt', '<RowHeadName>', REPLACE_TEXT);

REM Loop all files selected

StartLoop ([<File>:AB/Integ], 0);

$<$ Index $>=1$;

REM Get peak area values and write in text file

StartLoop (<Row $>, 2)$;

$<$ PeakArea $>=$ FromReportMatrix ([<File $>$ :AB/Integ], 1, 0, <Index $>, 3)$;

If $(<$ Index $>$, EQ., 1$)$;

$<$ PeakAreaLine $>=$ '<[,]PeakArea $>$ ';

Else ();

$<$ PeakAreaLine $>$ = '<PeakAreaLine $>\quad<$,]PeakArea $>$ ';

Endif 0;

$<$ Index $>=<$ Index $>+1$;

EndLoop (2);

TextToFile ('<Path>', '<FileName>.txt', '<PeakAreaLine>', APPEND_TEXT);

EndLoop (0);

D. Auto peak height - this code can extract peak height information from multiple spectra at a time and export into a .txt file.

\author{
VARIABLES SECTION \\ NUMERIC $<$ Row $>=1$; \\ STRING $<$ Path $>=$ "; \\ STRING <FileName $>=$ "; \\ STRING <PeakArea $>$ = "; \\ STRING <RowName $>$ = "; \\ STRING $<$ RowHeadName $>=$ "; \\ NUMERIC $<$ Index $>=1$; \\ STRING $<$ PeakAreaLine $>$ = "; \\ FILE $<$ File $>=$ AB $/$ Peak;
}

PROGRAM SECTION

REM This macro is used to export a number of peak height results to a text file. Developed by C.Yuan 
$<$ Path $>$ = GetOpusPath 0 ;

REM In the userdialog, select files, enter export file name, and path

UserDialog ('0', STANDARD, FILE:[<File>:AB/Peak], EDIT:'<FileName>', EDIT:'<Path>', BLANK, BLANK, BLANK, BLANK, BLANK, BLANK, BLANK, BLANK, BLANK, BLANK, BLANK);

REM Rowname is the peak name of integration,Row is the number of peaks

$<$ Row $>=$ FromReportHeader $([<$ File $>$ :AB $/$ Peak $], 1,0,3$, RIGHT);

StartLoop $(<$ Row $>, 1)$;

$<$ RowName $>=$ FromReportMatrix $([<$ File $>$ :AB $/$ Peak $], 1,0,<$ Index $>, 1)$;

If $(<$ Index $>$, EQ., 1);

$<$ RowHeadName $>$ = 'Frequency $<$ RowName $>$ ';

Else ();

$<$ RowHeadName $>$ = '<RowHeadName $>$ Frequency $<$ RowName $>$ ';

Endif 0;

$<$ Index $>=<$ Index $>+1$;

EndLoop (1);

REM Create a text file with a first line of peak name

TextToFile ('<Path>', '<FileName>.txt', '<RowHeadName>', REPLACE_TEXT);

REM Loop all files selected

StartLoop $([<$ File $>$ :AB/Peak $], 0)$;

$<$ Index> = 1;

REM Get peak height values and write in text file

StartLoop $(<$ Row $>, 2)$;

$<$ PeakArea $>=$ FromReportMatrix ([<File $>$ :AB/Peak $], 1,0,<$ Index $>, 2)$;

If $(<$ Index $>$, EQ., 1$)$;

$<$ PeakAreaLine $>=$ '<[,]PeakArea $>$ ';

Else ();

$<$ PeakAreaLine $>$ = '<PeakAreaLine $>\quad<$,]PeakArea $>$ ';

Endif 0 ;

$<$ Index $>=<$ Index $>+1$;

EndLoop (2);

TextToFile ('<Path>', '<FileName>.txt', '<PeakAreaLine>', APPEND_TEXT);

EndLoop (0);

\subsection{Mass Spectrometry Operation}

The mass spectrometer is a very sensitive measurement tool. Therefore the calibration of it is important before doing experiments. The QMS measures the ion currents of specific gas components, and then converts them to the partial pressure. Therefore, a sensitivity factor is needed for accurately measure the pressure with the QMS. The control program of the QMS stores two coefficient constants, one for the total pressure and one for the partial pressure. To obtain 
these constants, we introduce Ar gas into the chamber and read the pressure from the cold cathode gauge. Then input the pressure reading into the program and it will calculate and store the sensitivity factor. Although the ionization efficiency is different for different gases, the program use the same coefficient to convert the ion current to the pressure. Figure 9.1. shows the good consistency between the QMS pressure reading compared to the cold cathode gauge reading after the calibration is performed.

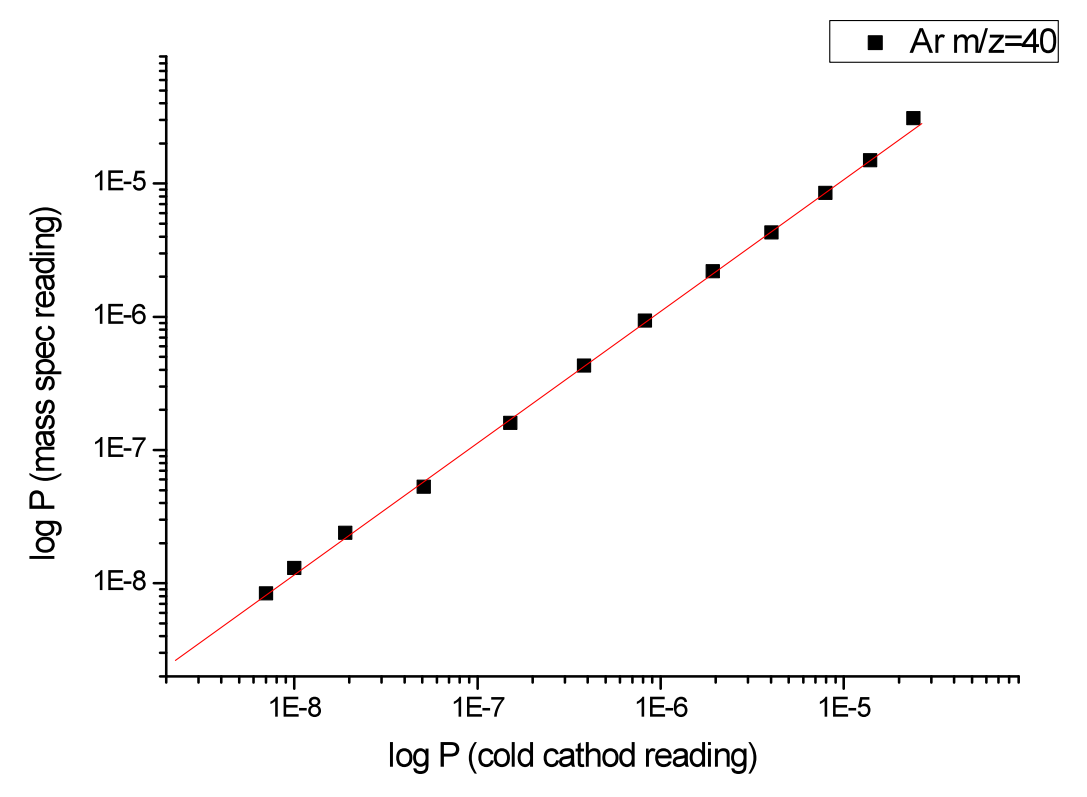

Figure 9.1: Comparison of QMS pressure reading and cold cathode pressure reading after QMS calibration.

The QMS also works with an electron multiplier, which can increase the gain by up to $10^{6}$. However, due to the limit of applied voltage (2500 V) and the properties of the multiplier, the highest gain achieved on our QMS is about $10^{5}$. To adjust the gain, one should choose a specific mass, manually input the desired gain, and the program will calculate the required voltage and adjust the gain. If the required voltage is beyond the limit, a failure signal will be returned. It should be noted that the upper limit of pressure that can be measured with the 
multiplier will decrease as the gain increases. Therefore, one should choose an appropriate gain to ensure the range of measurement as well as the sensitivity. The partial pressure detection limit of the QMS is $10^{-15}$ Torr as stated in the manual. In our operation, a lowest detection limit of $10^{-13}$ Torr can be achieved.

For unknown reason, the QMS sometimes displays spikes with a certain period as shown in Figure 9.2. It is found the problem may relate to the repeller cage or the filament, however, no practical method is found to solve the problem.

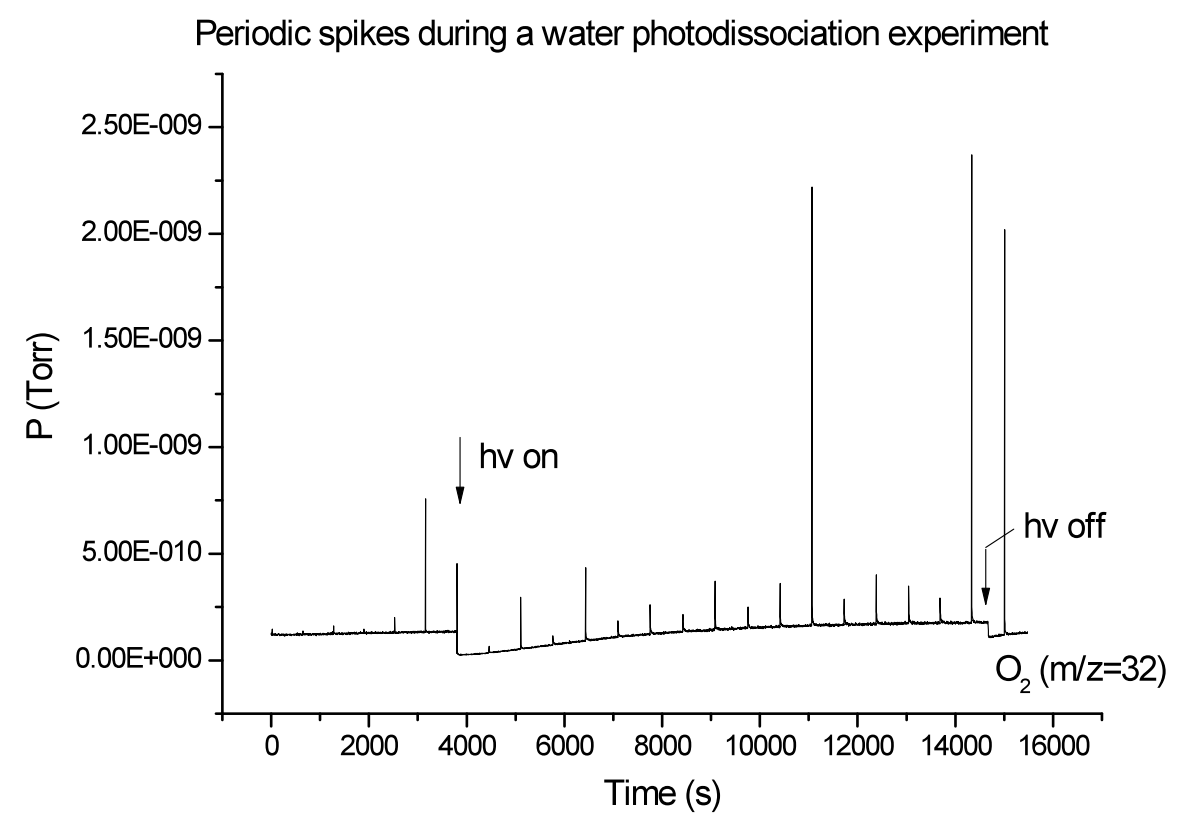

Figure 9.2: Periodic spikes observed by QMS during a water photodissociation experiment. The reason for this phenomenon is not clear.

\subsection{Design of Vacuum System}

The vacuum system contains a cubic stainless steel chamber, a dewar, several tubes, valves and pumps. In order to achieve a higher sensitivity of the QMS to pressure changes above the sample, I redesigned the vacuum system 
and moved the QMS much closer to the sample, as shown in Figure 9.3. The line-of-sight distance between the QMS and the sample is 2 ". The QMS sensitivity is increased by about 5 times after this modification.

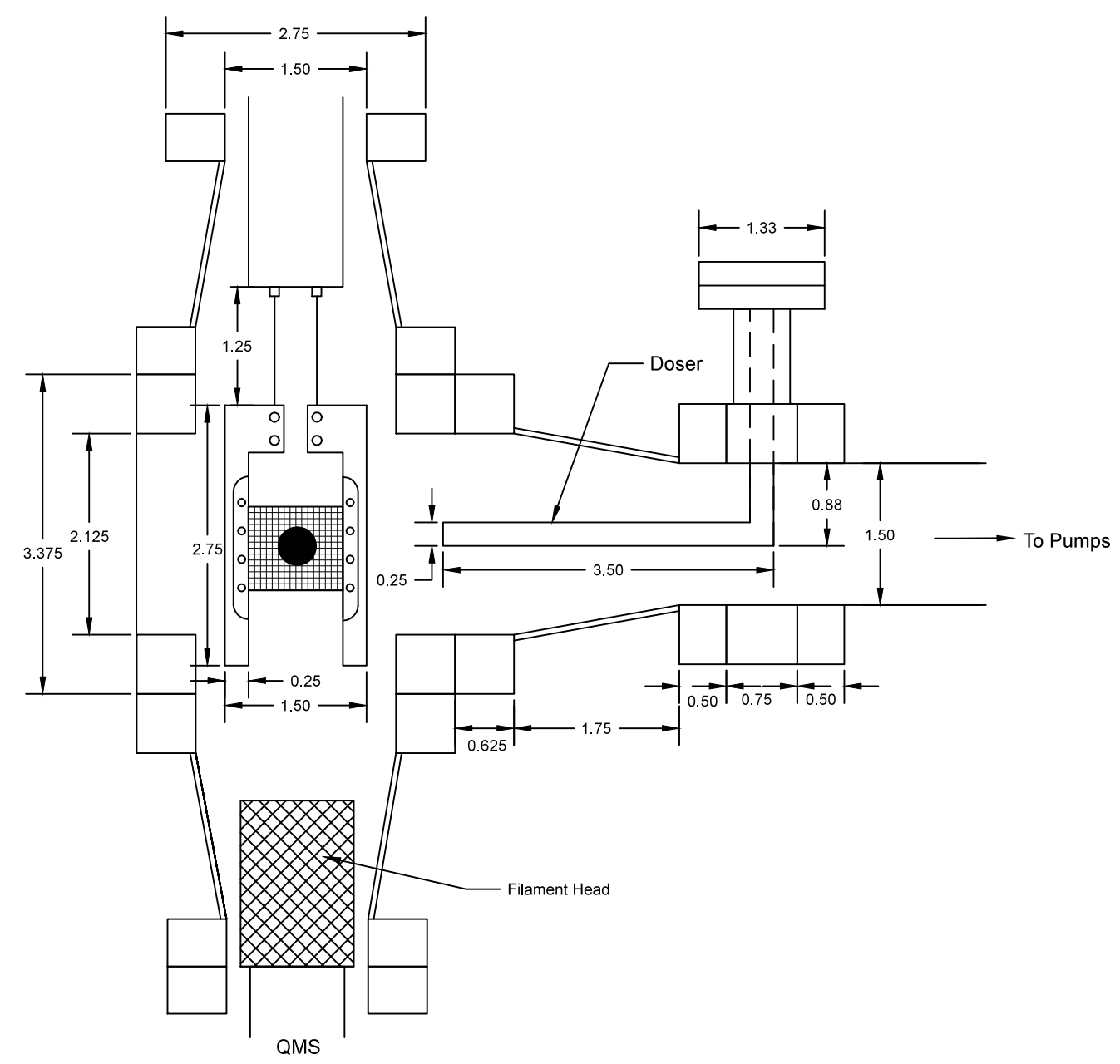

Figure 9.3: Design of vacuum system (all units in inches).

\subsection{Design of Dosing Crucible}

A dosing crucible which is used in $\mathrm{C}_{60}$ dosing was designed as shown in Figure 9.4. The crucible is made of Macor, a machineable ceramic material which 
can be heated to high temperatures. To heat the crucible, a tungsten wire is winded around the crucible, and connected to a $0-40 \mathrm{~V}$ d.c. power supply via a feedthrough mounted on a T-shaped flange. A type-K thermocouple is placed inside the crucible to measure the temperature. The temperature and heating power can be controlled by the Labview program which is also used in the sample temperature control.

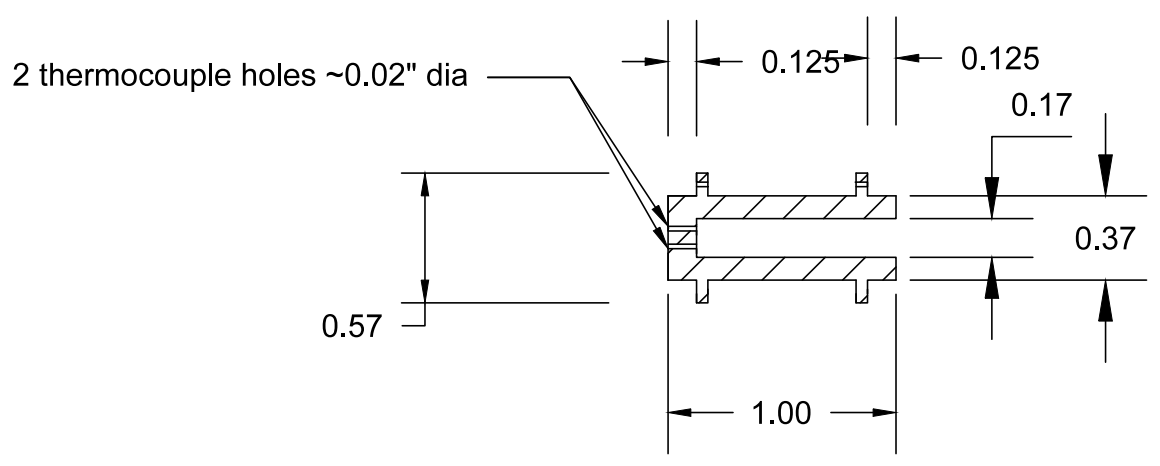

Side View

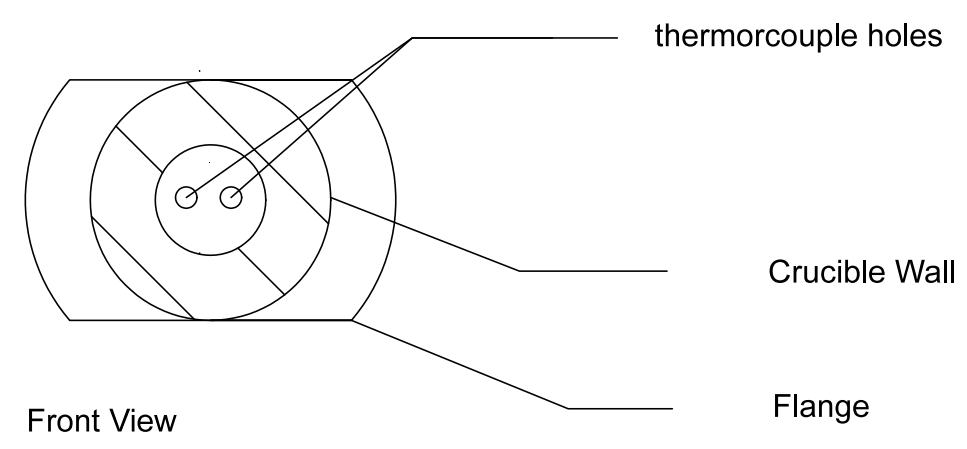

Figure 9.4: Design of dosing crucible (all units in inches).

To dose $\mathrm{C}_{60}$ onto the sample, we can place $\mathrm{C}_{60}$ powder in the crucible, and heat the crucible to the $\mathrm{C}_{60}$ sublimation temperature. During an experiment, it is found that $\mathrm{C}_{60}$ starts to sublime from above $600 \mathrm{~K}$, and an optimum sublimation temperature of $673 \mathrm{~K}$ is used in the dosing process. The support sample for $\mathrm{C}_{60}$ 
( $\mathrm{KBr}$ powder) is cooled with liquid $\mathrm{N}_{2}$ during the sublimation process, and is turned to a position that faces the crucible. Most $\mathrm{C}_{60}$ condenses on the sample, and some condenses on the sample holder, walls, and windows. The condensed $\mathrm{C}_{60}$ may be cleaned with acetone after the experiment is done.

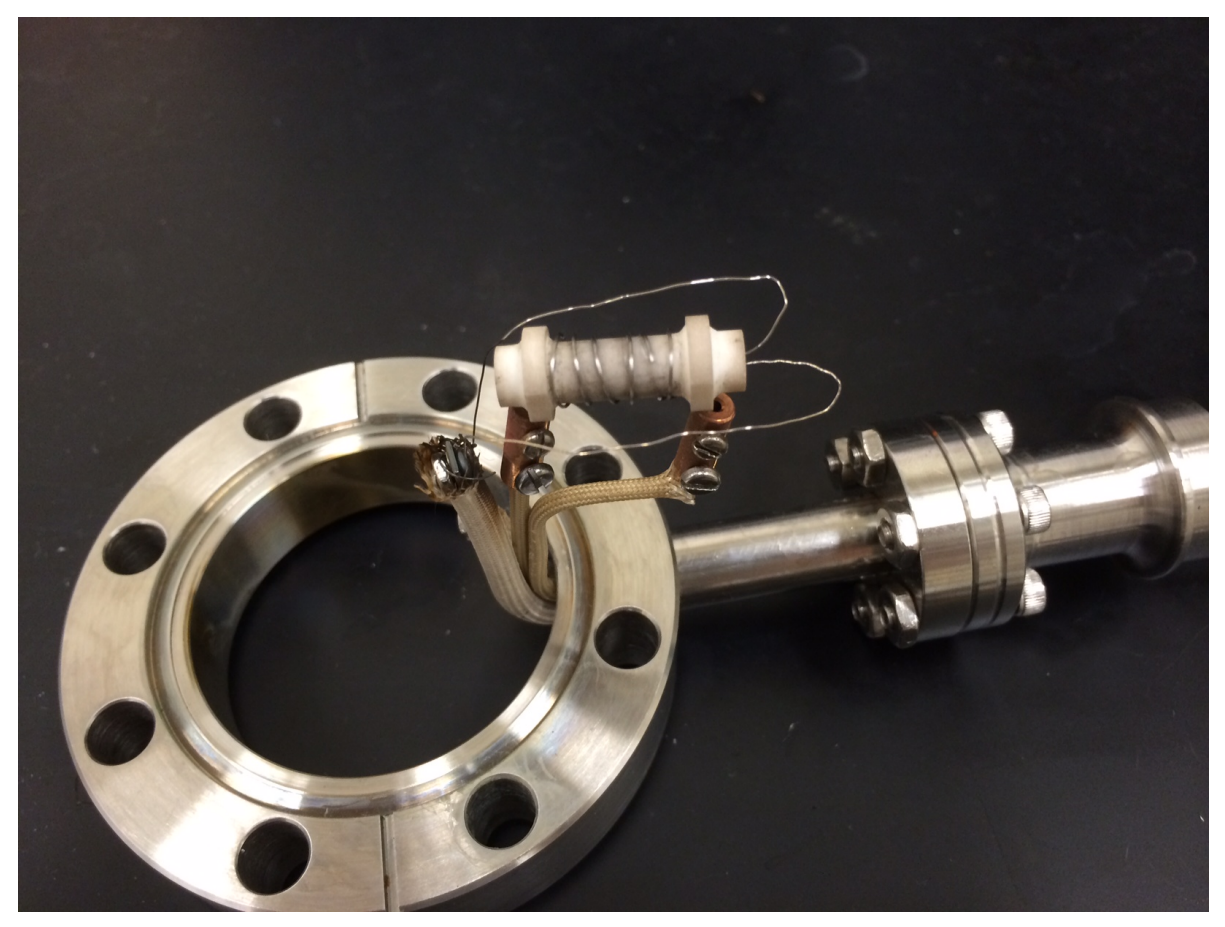

Figure 9.5: A picture showing the assembly of crucible with heating wires and thermocouple. 


\subsection{Design of Radiation Shield}

A radiation shield is designed to shield the Lyman- $\alpha$ radiation from scattering onto the dewar, as shown in Figure 9.6. This prevents photoprocesses from occurring in ices condensed on the dewar. The radiation shield is made of a stainless steel tube, with 4 drilled holes to allow the Lyman- $\alpha$ beam, the IR beam and the dosing beam to pass through.

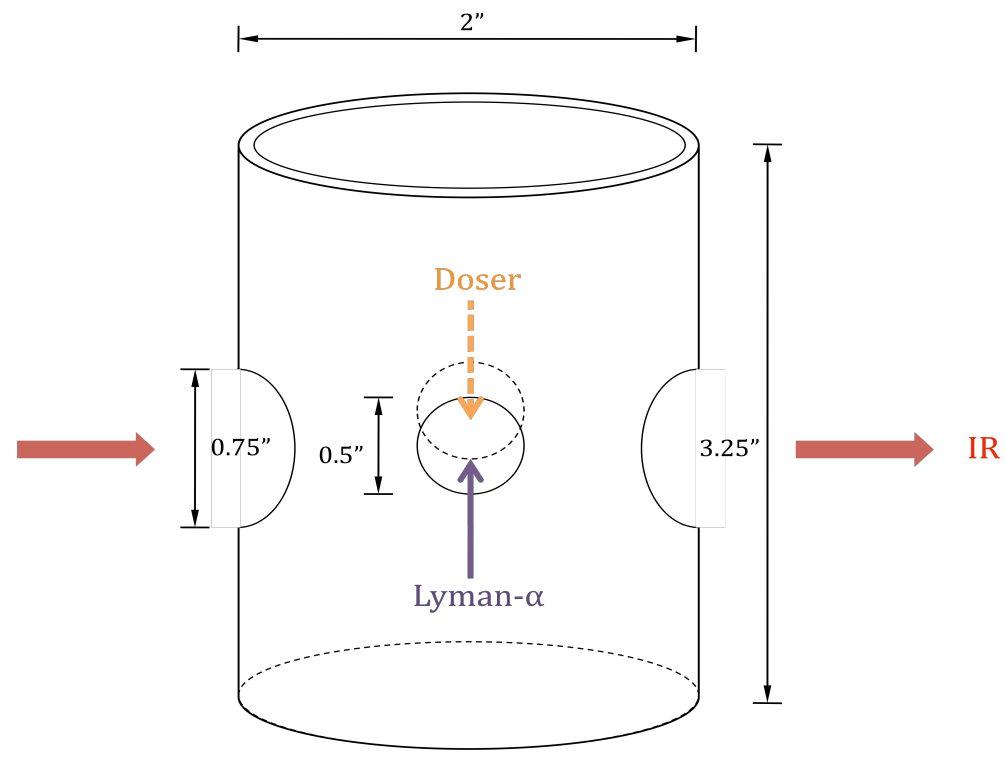

Figure 9.6: Design of radiation shield.

Experiments confirm that the radiation shield prevents about $70 \%$ scattered light on the dewar, although some scattering on the sample holder or the $\mathrm{W}$ grid cannot be shielded (Figure 9.7). The shield may slightly increase the $\mathrm{H}_{2}$ outgassing during Lyman- $\alpha$ irradiation because of its large surface area facing the lamp. 


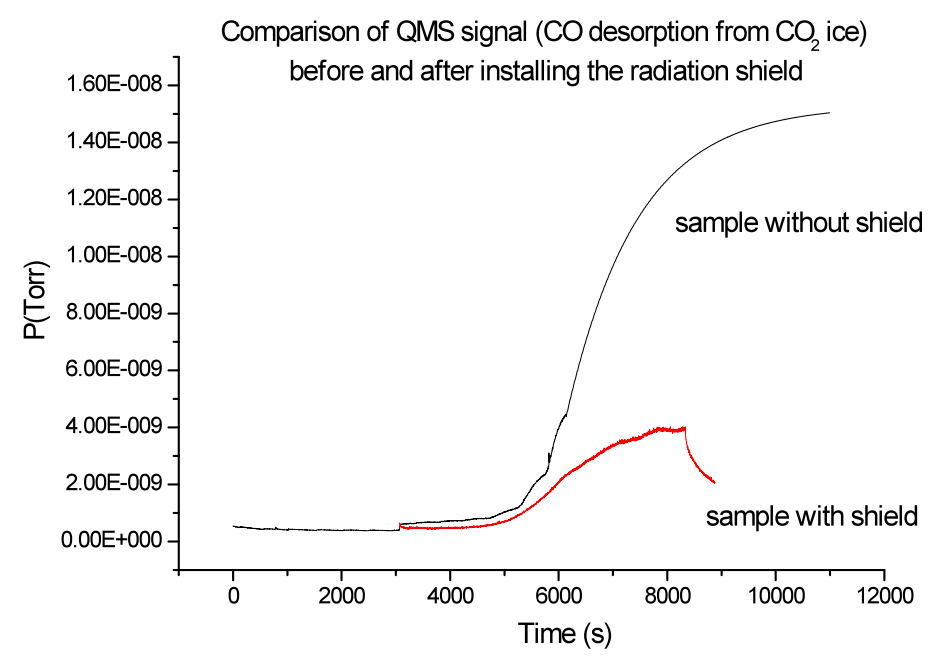

Figure 9.6: Comparison of QMS signal (CO desorption from $\mathrm{CO}_{2}$ ice) before and after installing the radiation shield.

\subsection{Reference}

1. Xu, J., H.J. Jansch, and J.T. Yates, Jr., Cryogenic trick for enhanced cooling using liquid nitrogen. J. Vac. Sci. Technol. A, 1993. 11: p. 726.

2. Rajappan, M., et al., Photochemical Decomposition of $\mathrm{N}_{2} \mathrm{O}$ by Lyman-a Radiation: Scientific Basis for a Chemical Actinometer. J. Phys. Chem. A, 2010. 114: p. 3443. 